\title{
Cochrane
}

Library

Cochrane Database of Systematic Reviews

\section{Carotid artery stenting versus endarterectomy for treatment of carotid artery stenosis (Review)}

Müller MD, Lyrer P, Brown MM, Bonati LH

Müller MD, Lyrer P, Brown MM, Bonati LH.

Carotid artery stenting versus endarterectomy for treatment of carotid artery stenosis.

Cochrane Database of Systematic Reviews 2020, Issue 1. Art. No.: CD000515.

DOI: 10.1002/14651858.CD000515.pub5.

www.cochranelibrary.com 
TABLE OF CONTENTS

HEADER

ABSTRACT

PLAIN LANGUAGE SUMMARY

SUMMARY OF FINDINGS

BACKGROUND

OBJECTIVES

METHODS

RESULTS

Figure 1.

DISCUSSION

AUTHORS' CONCLUSIONS

ACKNOWLEDGEMENTS

REFERENCES

\section{CHARACTERISTICS OF STUDIES}

DATA AND ANALYSES

Analysis 1.1. Comparison 1 Stenting or endarterectomy for symptomatic carotid stenosis, Outcome 1 Primary safety outcome: death or any stroke between randomisation and 30 days after treatment.

Analysis 1.2. Comparison 1 Stenting or endarterectomy for symptomatic carotid stenosis, Outcome 2 Death or any stroke between randomisation and 30 days after treatment according to age.

Analysis 1.3. Comparison 1 Stenting or endarterectomy for symptomatic carotid stenosis, Outcome 3 Death or any stroke between randomisation and 30 days after treatment according to sex.

Analysis 1.4. Comparison 1 Stenting or endarterectomy for symptomatic carotid stenosis, Outcome 4 Death or any stroke between randomisation and 30 days after treatment according to minimum pretrial experience in endovascular treatment of carotid stenosis.

Analysis 1.5. Comparison 1 Stenting or endarterectomy for symptomatic carotid stenosis, Outcome 5 Death or major or disabling stroke between randomisation and 30 days after treatment.

Analysis 1.6. Comparison 1 Stenting or endarterectomy for symptomatic carotid stenosis, Outcome 6 Death of any cause between randomisation and 30 days after treatment.

Analysis 1.7. Comparison 1 Stenting or endarterectomy for symptomatic carotid stenosis, Outcome 7 Any stroke between randomisation and 30 days after treatment.

Analysis 1.8. Comparison 1 Stenting or endarterectomy for symptomatic carotid stenosis, Outcome 8 Fatal, major, or disabling stroke between randomisation and 30 days after treatment.

Analysis 1.9. Comparison 1 Stenting or endarterectomy for symptomatic carotid stenosis, Outcome 9 Myocardial infarction between randomisation and 30 days after treatment.

Analysis 1.10. Comparison 1 Stenting or endarterectomy for symptomatic carotid stenosis, Outcome 10 Death or any stroke or myocardial infarction between randomisation and 30 days after treatment.

Analysis 1.11. Comparison 1 Stenting or endarterectomy for symptomatic carotid stenosis, Outcome 11 Primary combined safety and efficacy outcome: death or any stroke between randomisation and 30 days after treatment or ipsilateral stroke until the end of follow-up.

Analysis 1.12. Comparison 1 Stenting or endarterectomy for symptomatic carotid stenosis, Outcome 12 Death or any stroke between randomisation and end of follow-up.

Analysis 1.13. Comparison 1 Stenting or endarterectomy for symptomatic carotid stenosis, Outcome 13 Any stroke during follow-up (periprocedural events and people dying in the periprocedural period excluded).

Analysis 1.14. Comparison 1 Stenting or endarterectomy for symptomatic carotid stenosis, Outcome 14 Ipsilateral stroke during follow-up (periprocedural events and people dying in the periprocedural period excluded).

Analysis 1.15. Comparison 1 Stenting or endarterectomy for symptomatic carotid stenosis, Outcome 15 Death or any stroke or myocardial infarction between randomisation and 30 days after treatment or ipsilateral stroke until end of follow-up.

Analysis 2.1. Comparison 2 Stenting or endarterectomy for asymptomatic carotid stenosis, Outcome 1 Death or any stroke between randomisation and 30 days after treatment.

Analysis 2.2. Comparison 2 Stenting or endarterectomy for asymptomatic carotid stenosis, Outcome 2 Death or major or disabling stroke between randomisation and 30 days after treatment.

Analysis 2.3. Comparison 2 Stenting or endarterectomy for asymptomatic carotid stenosis, Outcome 3 Myocardial infarction between randomisation and 30 days after treatment. 
Analysis 2.4. Comparison 2 Stenting or endarterectomy for asymptomatic carotid stenosis, Outcome 4 Death or any stroke or myocardial infarction between randomisation and 30 days after treatment.

Analysis 2.5. Comparison 2 Stenting or endarterectomy for asymptomatic carotid stenosis, Outcome 5 Death or any stroke between randomisation and 30 days after treatment or ipsilateral stroke until end of follow-up.

Analysis 2.6. Comparison 2 Stenting or endarterectomy for asymptomatic carotid stenosis, Outcome 6 Death or any stroke or myocardial infarction between randomisation and 30 days after treatment or ipsilateral stroke until end of follow-up. .......... Analysis 3.1. Comparison 3 Stenting or endarterectomy in people with elevated surgical risk, Outcome 1 Death or any stroke between randomisation and 30 days after treatment.

Analysis 3.2. Comparison 3 Stenting or endarterectomy in people with elevated surgical risk, Outcome 2 Fatal, major, or disabling stroke between randomisation and 30 days after treatment.

Analysis 3.3. Comparison 3 Stenting or endarterectomy in people with elevated surgical risk, Outcome 3 Death of any cause between randomisation and 30 days after treatment.

Analysis 3.4. Comparison 3 Stenting or endarterectomy in people with elevated surgical risk, Outcome 4 Any stroke between randomisation and 30 days after treatment.

Analysis 3.5. Comparison 3 Stenting or endarterectomy in people with elevated surgical risk, Outcome 5 Myocardial infarction between randomisation and 30 days after treatment.

Analysis 3.6. Comparison 3 Stenting or endarterectomy in people with elevated surgical risk, Outcome 6 Death or any stroke or myocardial infarction between randomisation and 30 days after treatment.

Analysis 3.7. Comparison 3 Stenting or endarterectomy in people with elevated surgical risk, Outcome 7 Death or any stroke between randomisation and 30 days after treatment or ipsilateral stroke until end of follow-up.

Analysis 3.8. Comparison 3 Stenting or endarterectomy in people with elevated surgical risk, Outcome 8 Death or any stroke or myocardial infarction between randomisation and 30 days after treatment or ipsilateral stroke until end of follow-up. ........... Analysis 3.9. Comparison 3 Stenting or endarterectomy in people with elevated surgical risk, Outcome 9 Any stroke during follow-up (periprocedural events and people dying in the periprocedural period excluded).

Analysis 4.1. Comparison 4 Restenosis in endovascular treatment or endarterectomy for symptomatic or asymptomatic carotid stenosis, Outcome 1 Severe ( $\geq 70 \%$ ) restenosis during follow-up.

Analysis 4.2. Comparison 4 Restenosis in endovascular treatment or endarterectomy for symptomatic or asymptomatic carotid stenosis, Outcome 2 Moderate $(\geq 50 \%)$ or severe $(\geq 70 \%)$ restenosis during follow-up.

Analysis 5.1. Comparison 5 Access complications in stenting versus endarterectomy, Outcome 1 Cranial nerve palsy within 30 days of procedure.

Analysis 5.2. Comparison 5 Access complications in stenting versus endarterectomy, Outcome 2 Death or neurological complication up to 30 days after treatment.

Analysis 5.3. Comparison 5 Access complications in stenting versus endarterectomy, Outcome 3 Access site haematoma. ....... Analysis 6.1. Comparison 6 Endovascular treatment or medical care in people considered not suitable for surgery, Outcome 1 Death or any stroke between randomisation and end of follow-up.

Analysis 7.1. Comparison 7 Balloon angioplasty with/without stent insertion or endarterectomy for symptomatic carotid stenosis, Outcome 1 Death or any stroke between randomisation and 30 days after treatment.

Analysis 7.2. Comparison 7 Balloon angioplasty with/without stent insertion or endarterectomy for symptomatic carotid stenosis, Outcome 2 Any stroke during follow-up (periprocedural events and people dying in the periprocedural period excluded).

Analysis 7.3. Comparison 7 Balloon angioplasty with/without stent insertion or endarterectomy for symptomatic carotid stenosis, Outcome 3 Death or any stroke between randomisation and 30 days after treatment or ipsilateral stroke until the end of follow-up.

Analysis 7.4. Comparison 7 Balloon angioplasty with/without stent insertion or endarterectomy for symptomatic carotid stenosis, Outcome 4 Ipsilateral stroke during follow-up (periprocedural events and people dying in the periprocedural period excluded).

Analysis 7.5. Comparison 7 Balloon angioplasty with/without stent insertion or endarterectomy for symptomatic carotid stenosis, Outcome 5 Severe $(\geq 70 \%)$ restenosis during follow-up.

Analysis 8.1. Comparison 8 Balloon angioplasty with/without stent insertion or endarterectomy for asymptomatic carotid stenosis, Outcome 1 Death or any stroke between randomisation and 30 days after treatment.

Analysis 8.2. Comparison 8 Balloon angioplasty with/without stent insertion or endarterectomy for asymptomatic carotid stenosis, Outcome 2 Death or any stroke between randomisation and 30 days after treatment or ipsilateral stroke until the end of follow-up.

APPENDICES

WHAT'S NEW

HISTORY 
[Intervention Review]

\title{
Carotid artery stenting versus endarterectomy for treatment of carotid artery stenosis
}

Mandy D Müller¹, Philippe Lyrer¹, Martin M Brown², Leo H Bonati1,2

1Department of Neurology and Stroke Center, University Hospital Basel, Basel, Switzerland. 2Department of Brain Repair \&

Rehabilitation, UCL Institute of Neurology, London, UK

Contact address: Leo H Bonati, Department of Neurology and Stroke Center, University Hospital Basel, Petersgraben 4, Basel, 4031, Switzerland. bonatil@uhbs.ch.

Editorial group: Cochrane Stroke Group

Publication status and date: New search for studies and content updated (conclusions changed), published in Issue 1, 2020.

Citation: Müller MD, Lyrer P, Brown MM, Bonati LH. Carotid artery stenting versus endarterectomy for treatment of carotid artery stenosis. Cochrane Database of Systematic Reviews 2020, Issue 1. Art. No.: CD000515. DOI: 10.1002/14651858.CD000515.pub5.

Copyright ( 2020 The Cochrane Collaboration. Published by John Wiley \& Sons, Ltd.

\begin{abstract}
A B S T R A C T

\section{Background}

Carotid artery stenting is an alternative to carotid endarterectomy for the treatment of atherosclerotic carotid artery stenosis. This review updates a previous version first published in 1997 and subsequently updated in 2004, 2007, and 2012.
\end{abstract}

\section{Objectives}

To assess the benefits and risks of stenting compared with endarterectomy in people with symptomatic or asymptomatic carotid stenosis.

\section{Search methods}

We searched the Cochrane Stroke Group Trials Register (last searched August 2018) and the following databases: CENTRAL, MEDLINE, Embase, and Science Citation Index to August 2018. We also searched ongoing trials registers (August 2018) and reference lists, and contacted researchers in the field.

\section{Selection criteria}

Randomised controlled trials (RCTs) comparing stenting with endarterectomy for symptomatic or asymptomatic atherosclerotic carotid stenosis. In addition, we included RCTs comparing carotid artery stenting with medical therapy alone.

\section{Data collection and analysis}

One review author selected trials for inclusion, assessed trial quality and risk of bias, and extracted data. A second review author independently validated trial selection and a third review author independently validated data extraction. We calculated treatment effects as odds ratios (OR) and 95\% confidence intervals (CI), with endarterectomy as the reference group. We quantified heterogeneity using the $I^{2}$ statistic and used GRADE to assess the overall certainty of evidence.

\section{Main results}

We included 22 trials involving 9753 participants. In participants with symptomatic carotid stenosis, compared with endarterectomy stenting was associated with a higher risk of periprocedural death or stroke (the primary safety outcome; $\mathrm{OR} 1.70,95 \% \mathrm{Cl} 1.31$ to $2.19 ; \mathrm{P}<$ $0.0001, I^{2}=5 \% ; 10$ trials, 5396 participants; high-certainty evidence); and periprocedural death, stroke, or myocardial infarction (OR 1.43, $95 \% \mathrm{Cl} 1.14$ to $1.80 ; \mathrm{P}=0.002, \mathrm{I}^{2}=0 \% ; 6$ trials, 4861 participants; high-certainty evidence). The OR for the primary safety outcome was 1.11 $(95 \% \mathrm{Cl} 0.74$ to 1.64$)$ in participants under 70 years old and $2.23(95 \% \mathrm{Cl} 1.61$ to 3.08$)$ in participants 70 years old or more (interaction $\mathrm{P}$ $=0.007)$. There was a non-significant increase in periprocedural death or major or disabling stroke with stenting $(\mathrm{OR} 1.36,95 \% \mathrm{Cl} 0.97$ to $1.91 ; \mathrm{P}=0.08, \mathrm{I}^{2}=0 \% ; 7$ trials, 4983 participants; high-certainty evidence). Compared with endarterectomy, stenting was associated with 
lower risks of myocardial infarction (OR $0.47,95 \% \mathrm{Cl} 0.24$ to $\left.0.94 ; \mathrm{P}=0.03, \mathrm{I}^{2}=0 \%\right)$, cranial nerve palsy $(\mathrm{OR} 0.09,95 \% \mathrm{Cl} 0.06$ to $0.16 ; \mathrm{P}<$ $0.00001, \mathrm{I}^{2}=0 \%$ ), and access site haematoma (OR $0.32,95 \% \mathrm{Cl} 0.15$ to $0.68 ; \mathrm{P}=0.003, \mathrm{I}^{2}=27 \%$ ).

The combination of periprocedural death or stroke or ipsilateral stroke during follow-up (the primary combined safety and efficacy outcome) favoured endarterectomy (OR $1.51,95 \% \mathrm{Cl} 1.24$ to $1.85 ; \mathrm{P}<0.0001, \mathrm{I}^{2}=0 \% ; 8$ trials, 5080 participants; high-certainty evidence). The rate of ipsilateral stroke after the periprocedural period did not differ between treatments $\left(\mathrm{OR} 1.05,95 \% \mathrm{Cl} 0.75\right.$ to $\left.1.47 ; \mathrm{P}=0.77, \mathrm{I}^{2}=0 \%\right)$.

In participants with asymptomatic carotid stenosis, there was a non-significant increase in periprocedural death or stroke with stenting compared with endarterectomy (OR $1.72,95 \% \mathrm{Cl} 1.00$ to $2.97 ; \mathrm{P}=0.05, \mathrm{I}^{2}=0 \% ; 7$ trials, 3378 participants; moderate-certainty evidence). The risk of periprocedural death or stroke or ipsilateral stroke during follow-up did not differ significantly between treatments (OR 1.27 , $95 \% \mathrm{Cl} 0.87$ to $1.84 ; \mathrm{P}=0.22, \mathrm{I}^{2}=0 \% ; 6$ trials, 3315 participants; moderate-certainty evidence).

Moderate or higher carotid artery restenosis ( $50 \%$ or greater) or occlusion during follow-up was more common after stenting (OR $2.00,95 \%$ $\mathrm{Cl} 1.12$ to $\left.3.60 ; \mathrm{P}=0.02, \mathrm{I}^{2}=44 \%\right)$, but the difference in risk of severe restenosis was not significant $(70 \%$ or greater; OR $1.26,95 \% \mathrm{Cl} 0.79$ to $2.00 ; P=0.33, I^{2}=58 \%$; low-certainty evidence).

\section{Authors' conclusions}

Stenting for symptomatic carotid stenosis is associated with a higher risk of periprocedural stroke or death than endarterectomy. This extra risk is mostly attributed to an increase in minor, non-disabling strokes occurring in people older than 70 years. Beyond the periprocedural period, carotid stenting is as effective in preventing recurrent stroke as endarterectomy. However, combining procedural safety and longterm efficacy in preventing recurrent stroke still favours endarterectomy.

In people with asymptomatic carotid stenosis, there may be a small increase in the risk of periprocedural stroke or death with stenting compared with endarterectomy. However, Cls of treatment effects were wide and further data from randomised trials in people with asymptomatic stenosis are needed.

\section{PLAIN LANGUAGE SUMMARY}

\section{Metal tubes (stents) or surgery (endarterectomy) for treatment of carotid stenosis}

\section{Review question}

In people with narrowing of the carotid arteries, what are the risks and benefits of inserting metal tubes (stents) compared to surgical removal of the narrowing?

\section{Background}

Carotid stenosis, a narrowing of a major blood vessel in the neck carrying blood to the brain, can cause stroke. The standard treatment is to remove the narrowing by surgery, in which the surgeon opens the artery and removes the plaque (carotid endarterectomy). An alternative treatment (carotid artery stenting) uses a fine catheter tube which is passed through the skin and into the narrowed blood vessel. A metal tube (stent) is placed inside the vessel to prevent it narrowing again.

\section{Study characteristics}

We examined evidence about benefits and risks from studies that compared carotid artery stenting to carotid surgery in people who already had symptoms caused by carotid stenosis (stroke, transient ischaemic attack (TIA), or ocular (eye) symptoms) or in people who have never experienced symptoms. The studies had to be randomised; that is, the decision whether people were treated by stenting or surgery had to be made randomly and neither they nor the researchers were able to decide which treatment they received. This was to make the comparison as unbiased, or fair, as possible. We searched for studies up to August 2018. We assessed the quality of all the studies we included.

\section{Key results and conclusions}

This review included 22 studies involving 9753 participants.

In people who have already experienced symptoms from a narrowing in the carotid artery, stenting caused more strokes or deaths around the time of the procedure than surgery. This was especially true for people over the age of 70 years. After the initial procedure, both treatments were equally effective in preventing stroke or death in the long term.

In people who had never experienced symptoms from the carotid stenosis, both carotid artery stenting and surgery carried a similar risk of stroke or death in the short and long term, although the certainty of the evidence in these people was only moderate and the results should be interpreted with caution.

\section{Quality of the evidence}


In general, the quality of the evidence was high. The main factor reducing our confidence in the evidence was in studies comparing both treatments in people who had never experienced symptoms from the narrowing in the carotid artery. For these people, more studies are needed to draw firm conclusions about the risks and benefits of stenting compared to surgery. 
SUMMARY OF FINDINGS

Summary of findings for the main comparison. Carotid artery stenting compared to endarterectomy for symptomatic carotid stenosis

Carotid artery stenting compared to endarterectomy for symptomatic carotid stenosis

Patient or population: people with symptomatic carotid stenosis

Setting: hospital

Intervention: carotid artery stenting

Comparison: endarterectomy

\begin{tabular}{|c|c|c|c|c|c|c|}
\hline \multirow[t]{2}{*}{ Outcomes } & \multicolumn{2}{|c|}{ Anticipated absolute effects* $(95 \% \mathrm{Cl})$} & \multirow{2}{*}{$\begin{array}{l}\text { Relative effect } \\
(95 \% \mathrm{CI})\end{array}$} & \multirow{2}{*}{$\begin{array}{l}\text { № of partici- } \\
\text { pants } \\
\text { (studies) }\end{array}$} & \multirow{2}{*}{$\begin{array}{l}\text { Certainty of } \\
\text { the evidence } \\
\text { (GRADE) }\end{array}$} & \multirow[t]{2}{*}{ Comments } \\
\hline & $\begin{array}{l}\text { Risk with en- } \\
\text { darterectomy }\end{array}$ & $\begin{array}{l}\text { Risk with carotid artery } \\
\text { stenting }\end{array}$ & & & & \\
\hline \multirow{2}{*}{$\begin{array}{l}\text { Death or any stroke between randomi- } \\
\text { sation and } 30 \text { days after treatment }\end{array}$} & \multicolumn{2}{|c|}{ Study population } & \multirow{2}{*}{$\begin{array}{l}\text { OR } 1.70 \\
(1.31 \text { to } 2.19)\end{array}$} & \multirow{2}{*}{$\begin{array}{l}5396 \\
(10 \mathrm{RCTs})\end{array}$} & \multirow{2}{*}{$\begin{array}{l}\oplus \oplus \oplus \oplus \\
\text { High }\end{array}$} & \multirow[t]{2}{*}{-} \\
\hline & 44 per 1000 & $\begin{array}{l}72 \text { per } 1000 \\
(56 \text { to } 91)\end{array}$ & & & & \\
\hline \multirow{2}{*}{$\begin{array}{l}\text { Death or any stroke between randomi- } \\
\text { sation and } 30 \text { days after treatment or } \\
\text { ipsilateral stroke until end of follow-up }\end{array}$} & \multicolumn{2}{|c|}{ Study population } & \multirow{2}{*}{$\begin{array}{l}\text { OR } 1.51 \\
(1.24 \text { to } 1.85)\end{array}$} & \multirow{2}{*}{$\begin{array}{l}5080 \\
(8 \mathrm{RCTs})\end{array}$} & \multirow{2}{*}{$\begin{array}{l}\oplus \oplus \oplus \oplus \\
\text { High }\end{array}$} & \multirow[t]{2}{*}{-} \\
\hline & 70 per 1000 & $\begin{array}{l}102 \text { per } 1000 \\
(85 \text { to } 122)\end{array}$ & & & & \\
\hline \multirow{2}{*}{$\begin{array}{l}\text { Death or major or disabling stroke be- } \\
\text { tween randomisation and } 30 \text { days after } \\
\text { treatment }\end{array}$} & \multicolumn{2}{|c|}{ Study population } & \multirow{2}{*}{$\begin{array}{l}\text { OR } 1.36 \\
(0.97 \text { to } 1.91)\end{array}$} & \multirow{2}{*}{$\begin{array}{l}4983 \\
(7 \mathrm{RCTs})\end{array}$} & \multirow{2}{*}{$\begin{array}{l}\oplus \oplus \oplus \oplus \\
\text { High }\end{array}$} & \multirow[t]{2}{*}{-} \\
\hline & 24 per 1000 & $\begin{array}{l}33 \text { per } 1000 \\
(23 \text { to } 45)\end{array}$ & & & & \\
\hline \multirow{2}{*}{$\begin{array}{l}\text { Death or any stroke or myocardial in- } \\
\text { farction between randomisation and } 30 \\
\text { days after treatment }\end{array}$} & \multicolumn{2}{|c|}{ Study population } & \multirow{2}{*}{$\begin{array}{l}\text { OR } 1.43 \\
\text { (1.14 to } 1.80)\end{array}$} & \multirow{2}{*}{$\begin{array}{l}4861 \\
\text { (6 RCTs) }\end{array}$} & \multirow{2}{*}{$\begin{array}{l}\oplus \oplus \oplus \oplus \\
\text { High }\end{array}$} & \multirow[t]{2}{*}{-} \\
\hline & 56 per 1000 & $\begin{array}{l}78 \text { per } 1000 \\
(63 \text { to } 96)\end{array}$ & & & & \\
\hline
\end{tabular}

${ }^{\star}$ The risk in the intervention group (and its $95 \%$ confidence interval) is based on the assumed risk in the comparison group and the relative effect of the intervention (and its $95 \% \mathrm{Cl})$.

Cl: confidence interval; OR: odds ratio; $\mathbf{R C T}$ : randomised controlled trial.

GRADE Working Group grades of evidence

High certainty: we are very confident that the true effect lies close to that of the estimate of the effect. 


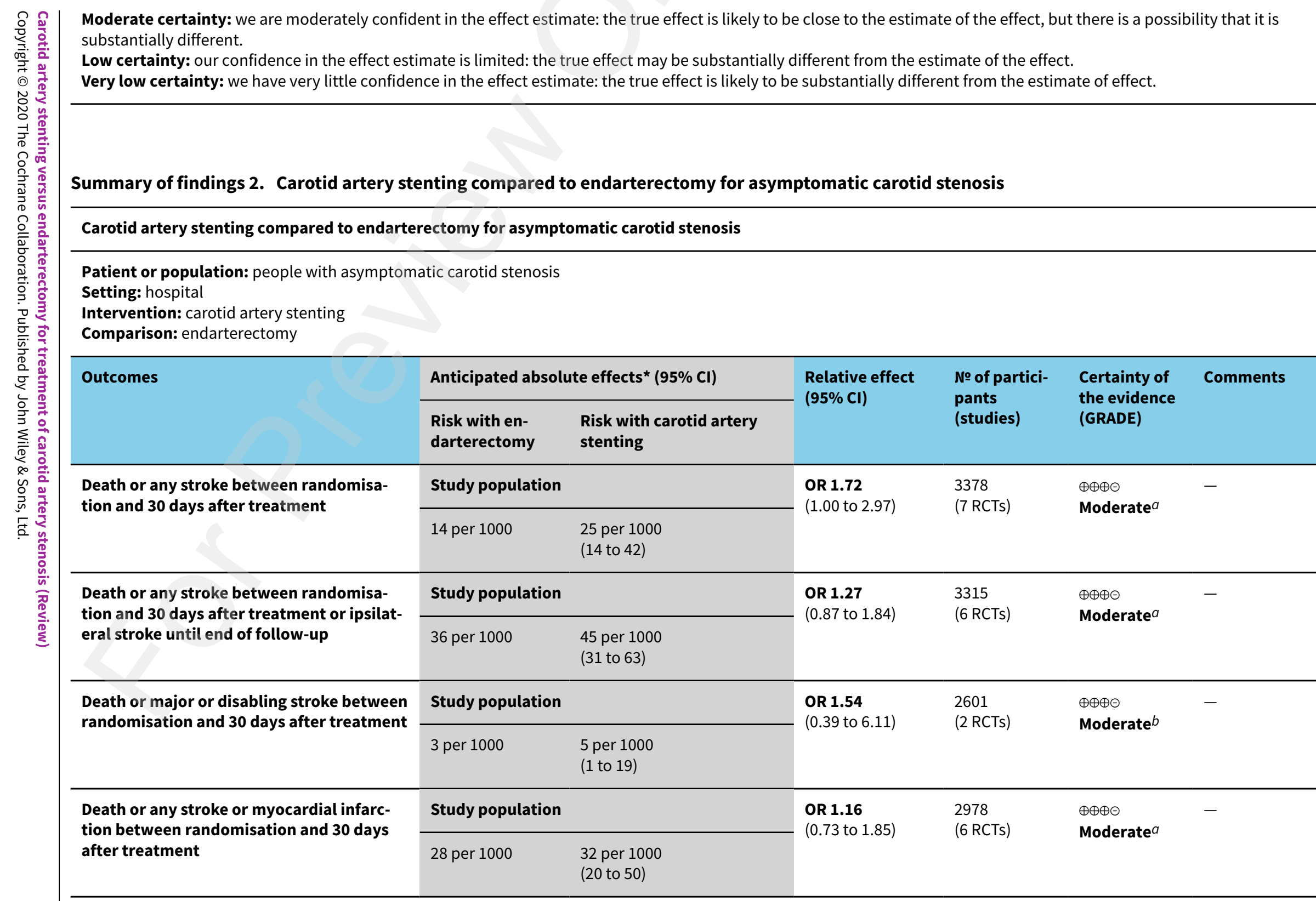

${ }^{\star}$ The risk in the intervention group (and its $95 \%$ confidence interval) is based on the assumed risk in the comparison group and the relative effect of the intervention (and its $95 \% \mathrm{Cl}$ ). 


\section{GRADE Working Group grades of evidence}

High certainty: we are very confident that the true effect lies close to that of the estimate of the effect.

Moderate certainty: we are moderately confident in the effect estimate: the true effect is likely to be close to the estimate of the effect, but there is a possibility that it is substantially different.

Low certainty: our confidence in the effect estimate is limited: the true effect may be substantially different from the estimate of the effect.

Very low certainty: we have very little confidence in the effect estimate: the true effect is likely to be substantially different from the estimate of effect.

aDowngraded one level due to wide confidence intervals surrounding the effect measures for the primary short- and long-term outcome in people with asymptomatic carotid stenosis.

bDowngraded one level as evidence was only available from two randomised trials.

\section{Summary of findings 3. Carotid artery stenting compared to endarterectomy for prevention of severe restenosis in people with symptomatic or} asymptomatic carotid stenosis

\section{Carotid artery stenting compared to endarterectomy for prevention of severe restenosis in people with symptomatic or asymptomatic carotid stenosis}

Patient or population: people with symptomatic or asymptomatic carotid stenosis

Setting: hospital

Intervention: carotid artery stenting

Comparison: endarterectomy

\begin{tabular}{|c|c|c|c|c|c|c|}
\hline \multirow[t]{2}{*}{ Outcomes } & \multicolumn{2}{|c|}{ Anticipated absolute effects* $(95 \% \mathrm{Cl})$} & \multirow{2}{*}{$\begin{array}{l}\text { Relative effect } \\
(95 \% \mathrm{Cl})\end{array}$} & \multirow{2}{*}{$\begin{array}{l}\text { № of partici- } \\
\text { pants } \\
\text { (studies) }\end{array}$} & \multirow{2}{*}{$\begin{array}{l}\text { Certainty of the } \\
\text { evidence } \\
\text { (GRADE) }\end{array}$} & \multirow[t]{2}{*}{ Comments } \\
\hline & Risk with endarterectomy & $\begin{array}{l}\text { Risk with carotid artery } \\
\text { stenting }\end{array}$ & & & & \\
\hline \multirow{2}{*}{$\begin{array}{l}\text { Severe ( } \geq 70 \%) \\
\text { restenosis during fol- } \\
\text { low-up }\end{array}$} & Study population & & \multirow{2}{*}{$\begin{array}{l}\text { OR } 1.21 \\
\text { (0.76 to } 1.93)\end{array}$} & \multirow{2}{*}{$\begin{array}{l}5744 \\
\text { (9 RCTs) }\end{array}$} & \multirow{2}{*}{$\begin{array}{l}\oplus \oplus \ominus \ominus \\
\text { Low }^{\mathrm{a}, \mathrm{b}}\end{array}$} & \multirow[t]{2}{*}{-} \\
\hline & 56 per 1000 & $\begin{array}{l}67 \text { per } 1000 \\
(43 \text { to } 103)\end{array}$ & & & & \\
\hline
\end{tabular}

*The risk in the intervention group (and its $95 \%$ confidence interval) is based on the assumed risk in the comparison group and the relative effect of the intervention (and its $95 \% \mathrm{Cl})$.

Cl: confidence interval; OR: odds ratio; $\mathbf{R C T}$ : randomised controlled trial.

\section{GRADE Working Group grades of evidence}

High certainty: we are very confident that the true effect lies close to that of the estimate of the effect.

Moderate certainty: we are moderately confident in the effect estimate: the true effect is likely to be close to the estimate of the effect, but there is a possibility that it is substantially different.

Low certainty: our confidence in the effect estimate is limited: the true effect may be substantially different from the estimate of the effect. 
bDowngraded one level due to wide confidence intervals of effect measures. 


\section{B A C K G R O U N D}

\section{Description of the condition}

Stenosis of the carotid artery is usually caused by focal atherosclerosis, typically at the site where the internal carotid artery - the main conduit of blood supplying the brain - originates from the common carotid artery. The prevalence of asymptomatic carotid stenosis ranges from $0.2 \%$ in men below the age of 50 years to $7.5 \%$ in men and $5.0 \%$ in women 80 years or older (De Weerd 2010). Carotid stenosis causes about $10 \%$ to $15 \%$ of ischaemic strokes (Petty 1999).

\section{Description of the intervention}

Carotid stenosis is conventionally treated by carotid endarterectomy. Multicentre randomised controlled trials have shown that endarterectomy significantly reduces the risk of ipsilateral stroke in people with severe symptomatic carotid stenosis. The European Carotid Surgery Trial (ECST) showed a reduction in ipsilateral stroke with symptoms lasting longer than seven days (including perioperative events) in the surgically treated participants from $20.6 \%$ to $6.8 \%$ at three-year follow-up ( $P<$ 0.0001) (ECST 1998). In the North American Symptomatic Carotid Endarterectomy Trial (NASCET), the risk of any ipsilateral stroke (again including perioperative events) was reduced from $26 \%$ to $9 \%$ after two years among participants with severe stenosis (70\% or greater narrowing; $P<0.001$ ) (NASCET 1991), and from $22.2 \%$ to $15.7 \%$ after five years among participants with moderate stenosis (50\% to $69 \%$ narrowing; $P=0.045$ ) (NASCET 1998). In the Asymptomatic Carotid Atherosclerosis Study (ACAS), surgery for asymptomatic carotid stenosis significantly reduced the overall five-year risk of ipsilateral stroke or any perioperative stroke or death from $11 \%$ to $5.1 \%(P=0.004)$, but not the risk of major ipsilateral stroke or any perioperative stroke or death $(6.5 \%$ in the medical group, $3.4 \%$ in the surgical group; $P=0.12$ ) (ACAS 1995). The Asymptomatic Carotid Surgery Trial (ACST) showed a significant reduction in the risk of any stroke or perioperative death from $10.9 \%$ to $6.9 \%(P=0.0001)$ after five years, and from $17.9 \%$ to $13.4 \%(P=0.009)$ after 10 years (ACST 2010).

Endovascular techniques for treating carotid stenosis by advancing a catheter inserted in the femoral artery were developed as an alternative to endarterectomy. Initially, percutaneous transluminal balloon angioplasty was used. Later, stents specifically designed for insertion in the carotid artery were invented and have been used to treat carotid stenosis either with or without prior balloon angioplasty. Primary stenting has replaced balloon angioplasty as the endovascular treatment most often used in clinical practice. Stenting might be safer in experienced hands than simple balloon angioplasty, because dissection and occlusion of the carotid artery are less likely to occur (Diethrich 1996; Roubin 2001). If dissection occurs, adverse consequences might be minimised, because the stent maintains laminar flow across the stenosis and seals the site of dissection, preventing a free intimal flap. Superior dilation achieved by stenting compared with balloon angioplasty might also reduce the risk of stroke in the early post-treatment period, and the rate of residual or recurrent stenosis. In the coronary circulation, stenting has been shown to produce superior outcomes compared with balloon angioplasty (Fischman 1994; Serruys 1996).

Carotid artery stenting may be a useful alternative to carotid endarterectomy. Potential advantages include avoidance of general anaesthesia, avoidance of an incision in the neck with the risk of cranial and cutaneous nerve damage, and a reduction in the rate of general complications of surgery, for example, myocardial infarction. Surgically inaccessible lesions can be treated and both the procedure and admission time are usually shorter than for surgery. However, carotid stenting does not remove the atherosclerotic lesion and may dislodge emboli during catheterisation, causing periprocedural stroke.

Some evidence on the safety and efficacy of carotid artery stenting stems from non-randomised registries. In one European registry, 1611 patients (441 symptomatic, 1170 asymptomatic) underwent carotid artery stenting with embolic protection in eight highvolume centres. Patients were followed for 30 days by either a neurologist or a National Institutes of Health Stroke Scale (NIHSS)certified interventionist. In this registry, any commercially available protection system and stent device could be used. In total, $99.7 \%$ of all patients were treated with an embolic protection device (EPD; 1607 participants), most of which were of the distal filter type. The risk of death or any stroke within 30 days of treatment was $1.36 \%$ in all patients combined. In total, $1.12 \%$ of all patients (18 participants) experienced a stroke within 30 days of treatment (Stabile 2012). A German registry, which analysed a total of 5869 carotid stenting procedures, also included both symptomatic (50\%) and asymptomatic patients. Overall, $82.8 \%$ of patients were treated with a protection device, most of which were of the distal filter type. In this registry, the stroke rate between treatment and patient discharge or transfer was $2.8 \%$ (160 participants), $1.3 \%$ sustained a minor stroke and $1.5 \%$ a major stroke. However, $60 \%$ of patients in this registry were not assessed by an independent neurologist and patients were followed only until discharge or transfer to another hospital (length of postinterventional hospital stay 2 to 11 days; Staubach 2014). Because of these differences in patient selection and follow-up, these figures are difficult to compare with data from randomised trials. The US Carotid Artery Revascularization and Endarterectomy Registry (CARE) analysed carotid artery stenting procedures performed between 2005 and 2008. In total, 5804 patients were analysed, 2801 of which had symptomatic carotid stenosis. Thirty-day outcomes were reported for $70 \%$ of the patients. The 30-day stroke rate for patients with available follow-up was $5.35 \%$, and the 30 -day stroke or death rate was $6.12 \%$. Outcomes were not reported according to symptom status (Anderson 2010).

\section{Technical aspects of treatment: cerebral protection devices, stent design, access route}

Concern regarding the risk of distal embolisation of debris being dislodged from the atheromatous plaque during stent deployment and resulting in neurological deficit has led to the introduction and increasing use of cerebral protection devices. This concept was first described in the series by Theron 1996. There are now many case series in the literature reporting experience of endovascular treatment with temporary cerebral protection, with reported complication rates ranging from $1 \%$ to $9 \%$ (Reimers 2001; Bonaldi 2002; Guimaraens 2002; Reimers 2004; Bush 2005; Gray 2006; Safian 2006; White 2006; CAPTURE 2007). In one systematic review of non-randomised case series including people with symptomatic and asymptomatic carotid stenosis, 30-day stroke or death rates were $1.8 \%$ in people stented with and $5.5 \%$ in people stented without protection devices $(P<0.001)$ (Kastrup 2003). Another systematic review reported higher rates of silent ischaemic brain lesions on diffusion-weighted magnetic resonance 
imaging (MRI) with unprotected stenting than with protected stenting (Schnaudigel 2008). However, some of the included studies used historical control groups or were prone to selection bias. Newer, randomised controlled trials comparing stenting with EPDs to unprotected stenting contradicted those findings. In the MRI substudy of the International Carotid Stenting Study (ICSS), participants were randomly assigned to endovascular treatment or endarterectomy. Brain MRI including diffusion-weighted imaging (DWI) to detect ischaemic brain lesions was performed in all participants before and after treatment. A subgroup analysis showed that participants who received endovascular treatment with cerebral protection devices of the distal filter type showed a higher incidence of new ischaemic brain lesions seen on DWI after treatment than participants treated with unprotected stenting (ICSS 2010). Two small randomised studies comparing stenting with embolic filter protection to unprotected stenting confirmed these results (Barbato 2008; Macdonald 2010). In addition, one must also consider that distal filter devices cannot prevent embolic events originating from the aortic arch occurring during the navigation of the arch in order to reach the lesion at the carotid bifurcation during carotid artery stenting with a femoral approach. In order to avoid this problem, transcervical approaches, where the common carotid artery is directly catheterised, have been proposed.

Proximal balloon occlusion devices constitute an alternative method of cerebral protection during endovascular treatment. A reversal of blood flow across the stenosis is installed before the lesion is crossed with the catheter which is aimed at preventing embolism to the brain. However, not all patients tolerate flow reversal in the carotid artery.

Only a small amount of randomised evidence comparing the different cerebral protection systems exists. One prospective, randomised, single-centre trial enrolled 62 participants with asymptomatic or symptomatic carotid stenosis who were randomly assigned to carotid artery stenting with proximal balloon occlusion or carotid artery stenting with distal filter protection. Participants were followed clinically and with MRI, including DWI, before and after treatment. The incidence of new ischaemic brain lesions seen on DWI after treatment was significantly higher in the distal filter group than in the balloon occlusion group ( $87.1 \%$ with distal filter versus $45.2 \%$ with balloon occlusion; $P=0.001$ ). This finding was independent of the symptom status of the treated participants (Bijuklic 2012). One systematic review and meta-analysis of eight randomised and non-randomised studies comparing distal filter devices with proximal balloon occlusion published in 2014 found a significantly lower incidence of new ischaemic brain lesion on DWI after treatment in participants treated with proximal balloon occlusion (effect size $-0.43,95 \% \mathrm{Cl}-0.8$ to $-0.02 ; \mathrm{I}^{2}=70.1 \%$; Stabile 2014). However, the studies included in this meta-analysis showed substantial heterogeneity and potential bias caused by different experience levels of the interventionist or different stent design cannot be excluded. To date, there is insufficient evidence to support the superiority of one protection device over the other and a larger randomised controlled trial is warranted to answer this question.

Direct transcervical catheterisation and stent placement have been proposed in order to avoid emboli originating from the aortic arch or in patients where a transfemoral access is not possible. In one non-randomised study comparing transcervical carotid stenting with flow reversal (31 participants) versus transfemoral carotid stenting with distal filter protection devices (33 participants) showed a significantly lower rate of new ischaemic brain lesions on DWI after the procedure in participants treated with transcervical carotid artery stenting (12.9\%) compared to transfemoral carotid artery stenting (33.3\%) (Leal 2012). Other studies evaluating the safety and efficacy of transcervical carotid stenting with flow reversal also showed low 30-day stroke rates (Alvarez 2012; ROADSTER 2015). However, these were single-arm studies without a control group. One large randomised controlled trial comparing transcervical carotid artery stenting to transfemoral carotid artery stenting is warranted to support the superiority of this new method over transfemoral carotid artery stenting.

To minimise embolisation of plaque debris to the brain, closedcell stent devices with better coverage of the atherosclerotic plaque have been developed. However, only a small amount of randomised evidence is available comparing open-cell stents versus closed-cell stents. One randomised trial comparing opencell stents with closed-cell stents enrolled 96 participants with symptomatic (76 participants) and asymptomatic (20 participants) carotid stenosis. Participants were followed clinically and with DWMRI after the procedure. New DWI lesions were found significantly more often in participants treated with open-cell stents (24 participants) than in participants treated with closed-cell stents (12 participants; $P=0.002$ ). There was no significant difference in the 30-day stroke or death rate between the two groups. However, numbers were small and a real difference might have been missed due to a lack of power (Park 2013). In another study, 40 participants (17 with symptomatic carotid stenosis, 23 with asymptomatic carotid stenosis) were randomised in a 1:1 ratio to carotid artery stenting with an open-cell (Acculink) stent versus carotid artery stenting with a closed-cell (Xact) stent. Participants were followed clinically and with preprocedural and postprocedural DW-MRI. There was no significant difference in the rate of new DWI lesions after treatment between participants treated with opencell (53\%) and those treated with closed-cell stents (47\%). In this trial, only one participant treated with an open-cell stent whose postprocedural MRI did not show any new ischaemic lesions on DWI experienced a hemispheric stroke three days after treatment (Timaran 2011). In recent years, mesh-covered stents providing even better coverage of the atherosclerotic plaque have been introduced (Schofer 2015). However, to date there is no evidence available form randomised trials to support the superiority of these newer stent devices over others.

The main aim of treating carotid stenosis is the prevention of stroke in the long term. Carotid endarterectomy is effective at preventing ipsilateral stroke over long-term follow-up periods of 10 years or longer (ECST 1998; ACST 2010). To provide an alternative, carotid stenting needs to have similar long-term effectiveness. In the last few years, several large randomised controlled trials published results of extended follow-up providing evidence on the comparative long-term effectiveness of endarterectomy and endovascular treatment at preventing stroke (CREST 2010; EVA-3S 2006; ICSS 2010; Kentucky 2001; Kentucky 2004).

Because of the lack of large randomised trials with clinical outcomes, comparisons of protection devices, stent designs, and access routes for endovascular treatment have been omitted from the present review. 


\section{Why it is important to do this review}

Only randomised trials can answer the question whether carotid stenting is equivalent to endarterectomy in people fit for surgery or better than medical care in people unfit for surgery - in terms of treatment safety and long-term prevention of stroke in people with carotid stenosis. Therefore, we aimed to systematically review all randomised controlled trials comparing carotid angioplasty and stenting with carotid endarterectomy or medical care. The present review updates a previous version first published in 1997 (Crawley 1997) and subsequently updated in 2004 (Coward 2004), 2007 (Ederle 2007), and 2012 (Bonati 2012). In the 2012 update, there was sufficient evidence to compare treatment risks and shortterm efficacy between carotid stenting and endarterectomy for symptomatic carotid stenosis. However, data on long-term efficacy and for treatment of people with asymptomatic carotid stenosis were sparse. Since the last update in 2012, five previously identified trials published results of extended follow-up periods (Kentucky 2001; Kentucky 2004; EVA-3S 2006; CREST 2010; ICSS 2010), and six new randomised trials were identified which completed randomisation and published their results (Beijing 2013; Houston 2014; Ostrava 2014; ACT-1 2016; SPACE-2 2016; Carmel Medical Center 2017). The number of asymptomatic participants available for comparison was more than doubled. Thus, an update of this review became necessary.

\section{O B JECT IVES}

To assess the benefits and risks of stenting compared with endarterectomy in people with symptomatic or asymptomatic carotid stenosis.

\section{METHODS}

\section{Criteria for considering studies for this review}

\section{Types of studies}

We attempted to identify all unconfounded, truly randomised trials comparing carotid stenting with conventional carotid endarterectomy, and also trials comparing carotid stenting with medical therapy alone. We included trials in which the exact method of randomisation was still uncertain after communication with the authors. We excluded studies of carotid revascularisation procedures without control groups and studies without random allocation of treatment.

\section{Types of participants}

We considered trials including participants of any age or either sex with symptomatic or asymptomatic carotid stenosis eligible for inclusion in the review.

\section{Types of interventions}

We reviewed trials that allowed any acceptable technique for carotid endarterectomy (e.g. use of a shunt or not, patching or not, local or general anaesthesia) and which allowed any acceptable endovascular technique for treatment of carotid stenosis (e.g. simple balloon angioplasty, use of a stent or not, any type of cerebral protection device).

\section{Types of outcome measures}

We identified the following primary and secondary outcome measures.

\section{Primary outcomes}

- Primary outcome measure for evaluation of treatment safety was the combined outcome of death or any stroke occurring between randomisation and 30 days after treatment. For participants who did not undergo carotid revascularisation, this period was defined as the first 30 days after randomisation, or according to the definition used in the trial.

- Primary outcome measure for evaluation of combined safety and long-term efficacy was the combined outcome of death or any stroke occurring between randomisation and 30 days after treatment, or ipsilateral stroke occurring thereafter until the end of follow-up.*

${ }^{*}$ Changed or added at the time of the previous updates (Ederle 2007; Bonati 2012).

\section{Secondary outcomes}

- Secondary safety outcome measures included the following events occurring between randomisation and 30 days after treatment:

* combined outcome of death, or major or disabling stroke*;

* death of any cause ${ }^{\star \star}$;

* any stroke ${ }^{\star \star}$;

* combined outcome of fatal, major, or disabling stroke*;

* myocardial infarction*;

* combined outcome of death or any stroke or myocardial infarction $^{\star \star}$;

* cranial nerve palsy**;

* combined outcome of death or neurological complications (including stroke and cranial nerve palsy) ${ }^{\star *}$;

* access site haematoma (including cervical haematoma arising from surgical incision in endarterectomy or groin haematoma arising from skin puncture in endovascular treatment) requiring surgery, blood transfusion, or prolonging hospital stay*.

- Secondary efficacy outcome measures were:

* combined outcome of death or any stroke occurring between randomisation and end of follow-up ${ }^{\star \star}$;

* combined outcome of death or any stroke or myocardial infarction occurring between randomisation and 30 days after treatment or ipsilateral stroke until the end of followup $^{\star \star}$;

* ipsilateral stroke occurring during follow-up (excluding strokes occurring between randomisation and 30 days after treatment, and excluding participants dying within this period) ${ }^{\star \star}$;

* any stroke occurring during follow-up (excluding strokes occurring between randomisation and 30 days after treatment, and excluding participants dying within this period) ${ }^{\star \star}$;

* severe restenosis equivalent to or greater than $70 \%$ luminal narrowing according to the measurement used in the NASCET 1991 trial, determined by Doppler or duplex sonography, catheter angiography, or non-invasive (MRI or computer tomography) angiography at defined intervals ${ }^{\star \star}$;

* moderate or severe restenosis equivalent to or greater than $50 \%$ luminal narrowing according to the measurement used 
in the NASCET 1991 trial, determined by Doppler or duplex sonography*;

* cognitive performance after endarterectomy or endovascular therapy ${ }^{\star *}$.

${ }^{*}$ Changed or added at the time of the previous updates (Ederle 2007; Bonati 2012).

${ }^{\star \star}$ Changed or added for this current update.

\section{Outcome event definitions}

Stroke was defined as an acute deficit of focal neurological function with symptoms lasting for longer than 24 hours, resulting from intracranial vascular disturbance (ischaemia or haemorrhage). Visual loss, resulting from retinal ischaemia that lasted for longer than 24 hours, was included within the category of stroke (CSTC 2010). Stroke was classified as disabling if leading to a loss of functional independence, characterised by a score of 3 or more on the modified Rankin scale (mRS; Van Swieten 1988) or the Oxfordshire Handicap Stroke scale (Bamford 1989). If no measure of disability was provided we included major stroke, according to the definition used in the trials, in the combined category of disabling or major stroke.

We expected differences between trials in definitions of disabling or major stroke, myocardial infarction, and in the severity of cranial nerve palsy and access site haematoma required in order to qualify as an outcome event. We included these outcome events as reported in the trials.

\section{Search methods for identification of studies}

See the 'Specialized register' section on Cochrane Stroke's website (www.dcn.ed.ac.uk/csrg/entity/searchmethods.pdf). We searched for trials in all languages and arranged for the translation of relevant articles where necessary.

\section{Electronic searches}

We searched the Cochrane Stroke Group Trials Register in August 2018. In addition, we searched the following bibliographic databases: Cochrane Central Register of Controlled Trials (CENTRAL) (the Cochrane Library 2018, Issue 5) (Appendix 1), MEDLINE Ovid (1950 to August 2018) (Appendix 2), Embase Ovid (1980 to August 2018) (Appendix 3), and Science Citation Index (1945 to August 2018) (Appendix 4).

We developed the MEDLINE search strategy with the help of the Cochrane Stroke Group Information Specialist and adapted it for the other databases.

We also searched the following ongoing trials registers (August 2018):

- Stroke Trials Registry (www.strokecenter.org/trials/; Appendix 5);

- US National Institutes of Health Ongoing Trials Register ClinicalTrials.gov (www.clinicaltrials.gov/; Appendix 6);

- ISRCTN Registry (www.isrctn.com/; Appendix 7);

- World Health Organization International Clinical Trials Registry Platform (apps.who.int/trialsearch; Appendix 8).

\section{Searching other resources}

We searched reference lists of relevant articles and included studies, and contacted individuals active in the field for additional information when necessary.

\section{Data collection and analysis}

\section{Selection of studies}

One review author (MDM) screened titles and abstracts of the records identified from the searches of the electronic bibliographic databases and excluded obviously irrelevant studies. We obtained the full text of the remaining studies and one review author (MDM) selected trials for inclusion, assessed trial quality and risk of bias, and extracted data. A second review author independently validated trial selection (LHB) and a third review author independently validated data extraction (PL). We resolved disagreements by discussion and consultation with the other authors if necessary.

\section{Data extraction and management}

One review author (MDM) extracted trial data. A second review author (PL) independently validated extracted trial data. We resolved disagreements by consensus. We identified data in published articles and sought additional information from the principal investigators of the included trials. When review authors had a personal interest in clinical trials (e.g. they were investigators or members of steering committees), they were not involved in data extraction and were replaced by another review author.

We had access to individual participant data from six trials (CAVATAS-CEA 2001; EVA-3S 2006; SPACE 2006; BACASS 2008; CREST 2010; ICSS 2010), and used reported outcomes of individual participants from two other trials (Leicester 1998; Ostrava 2014), to perform subgroup analyses. For trials where access to individual participant data was available, we extracted short-term outcome events used for comparison of treatment safety according to the definition of the periprocedural period used in this review (i.e. events occurring between randomisation and 30 days after treatment).

We extracted the following data:

- number of symptomatic and asymptomatic participants originally allocated to each treatment group to allow an intention-to-treat analysis;

- inclusion and exclusion criteria;

- mean age and sex distribution of study participants;

- mean degree of stenosis of the relevant carotid artery at randomisation;

- intervention characteristics, including technical aspects of procedures (endovascular: balloon angioplasty alone or routine insertion of stents, use of protection devices, predilation and postdilation of the stenosis; endarterectomy: surgical method, type of anaesthesia, use of patches, shunting) and periprocedural medications;

- level of experience required from interventional physicians and surgeons performing the procedures, expressed as the number of endovascular procedures or endarterectomies for treatment of carotid stenosis performed before the trial; 
- outcome measures as specified under Types of outcome measures;

- definitions of outcome measures;

- nature and intervals of follow-up assessments.

\section{Assessment of risk of bias in included studies}

One review author (MDM) assessed risk of bias in included studies using the tool recommended by Cochrane (Higgins 2011). A second review author $(\mathrm{PL})$ independently validated assessment of risk of bias. We resolved disagreements by consensus.

We extracted, then analysed, the following trial data to assess risk of bias:

- method of generation of a random sequence of treatment assignments;

- whether the randomising doctor might have anticipated the allocated treatment (allocation concealment);

- whether participants were followed up by study personnel who were not involved in the revascularisation procedures and whether outcome events were adjudicated blinded to treatment;

- number of participants excluded from analysis or lost to followup;

- whether the reported analyses had been prespecified in a study protocol.

\section{Assessment of heterogeneity}

We quantified heterogeneity among trial results using the $1^{2}$ statistic. We considered a value greater $50 \%$ as representing substantial heterogeneity (Higgins 2011). If significant heterogeneity was present, we explored possible reasons for heterogeneity and identified if there were clinical or methodological explanations for differences in treatment effects between studies.

\section{Data synthesis}

We analysed outcomes following the intention-to-treat principle; that is, we compared all participants who were randomised and in whom any information on outcome was reported according to their randomly assigned treatment, irrespective of whether they received this treatment or not.

For trials comparing endovascular treatment with endarterectomy, we analysed separately the data from participants with symptomatic and asymptomatic carotid stenosis and data from trials enrolling participants considered at increased surgical risk, whenever possible. For the outcome measures cranial nerve palsy (and its combination with stroke or death) and access site haematoma, we provided pooled treatment effects including all trials with available data because we did not expect any difference in treatment effects according to symptom status or surgical risk. However, we also analysed separately the treatment effects for participants with asymptomatic carotid stenosis or those perceived to be at increased risk for vascular events with surgery.

We analysed summary data of all participants randomised and analysed in the included studies using Mantel-Haenszel random-effect models. We reported the treatment effects as odds ratios (OR), that is, the odds of an unfavourable outcome in participants treated by endovascular intervention compared with the corresponding odds in participants treated surgically or medically, with a $95 \%$ confidence interval $(\mathrm{Cl})$. We chose $\mathrm{P}<0.05$ as the level of significance.

\section{Subgroup analysis and investigation of heterogeneity}

Among the eight trials with available individual participant data, we calculated the OR for the primary safety outcome measure separately for participants who were aged 70 years or older (which was at or near the mean age of the participant populations of most included trials) and younger participants (Leicester 1998; CAVATAS-CEA 2001; EVA-3S 2006; SPACE 2006; BACASS 2008; CREST 2010; ICSS 2010; Ostrava 2014). We also performed subgroup analysis by sex including these eight trials. In addition, we investigated for heterogeneity according to the required number of carotid endovascular procedures interventionists needed to have performed before joining the trials, separating the trials at an arbitrary cut-off of up to 10 procedures, or more. We formally tested interactions between treatment effect and subgroup variables using a standard test for heterogeneity across subgroup results (Higgins 2011).

\section{GRADE assessment and 'Summary of findings' tables}

We used the GRADEpro Guideline Development Tool to create 'Summary of findings' tables for the comparison of stenting versus endarterectomy for participants with symptomatic carotid stenosis and participants with asymptomatic carotid stenosis (Summary of findings for the main comparison; Summary of findings 2). These tables present the results and the certainty of the evidence of the main outcomes, using the GRADE system, which classifies the certainty of the evidence as high, moderate, low, and very low. The outcomes included in the 'Summary of findings' tables are for participants with symptomatic carotid stenosis (Summary of findings for the main comparison) and for participants with asymptomatic carotid stenosis (Summary of findings 2):

- primary safety outcome (death or any stroke between randomisation and 30 days after treatment);

- primary combined safety and long-term efficacy outcome (death or any stroke between randomisation and 30 days after treatment or ipsilateral stroke until the end of follow-up).

The GRADE approach appraises the certainty of the body of evidence according to the extent to which one can be confident that an estimate of effector association reflects the item assessed. The certainty of a body of evidence is based on within-study risk of bias (methodological quality), directness of the evidence, heterogeneity of the data, precision of effect estimates, and risk of publication bias.

\section{RE S U L T S}

\section{Description of studies}

\section{Results of the search}

We found 22 randomised controlled trials involving 9753 participants with available outcome data that fulfilled the inclusion criteria (Figure 1). Twelve of the trials either completed enrolment of the planned number of study participants, or no sample size calculation was performed or reported (CAVATAS-CEA 2001; Kentucky 2001; Beijing 2003; Kentucky 2004; TESCAS-C 2006; BACASS 2008; Beijing 2009; CAVATAS-MED 2009; CREST 2010; ICSS 
2010; Beijing 2013; Ostrava 2014), while eight trials terminated recruitment before the originally planned minimum sample size was reached (Leicester 1998; WALLSTENT 2001; SAPPHIRE 2004;
EVA-3S 2006; SPACE 2006; Regensburg 2008; ACT-1 2016; SPACE-2 2016). Details of study design are provided in the Characteristics of included studies table and summarised here. 
Figure 1. Study flow diagram.

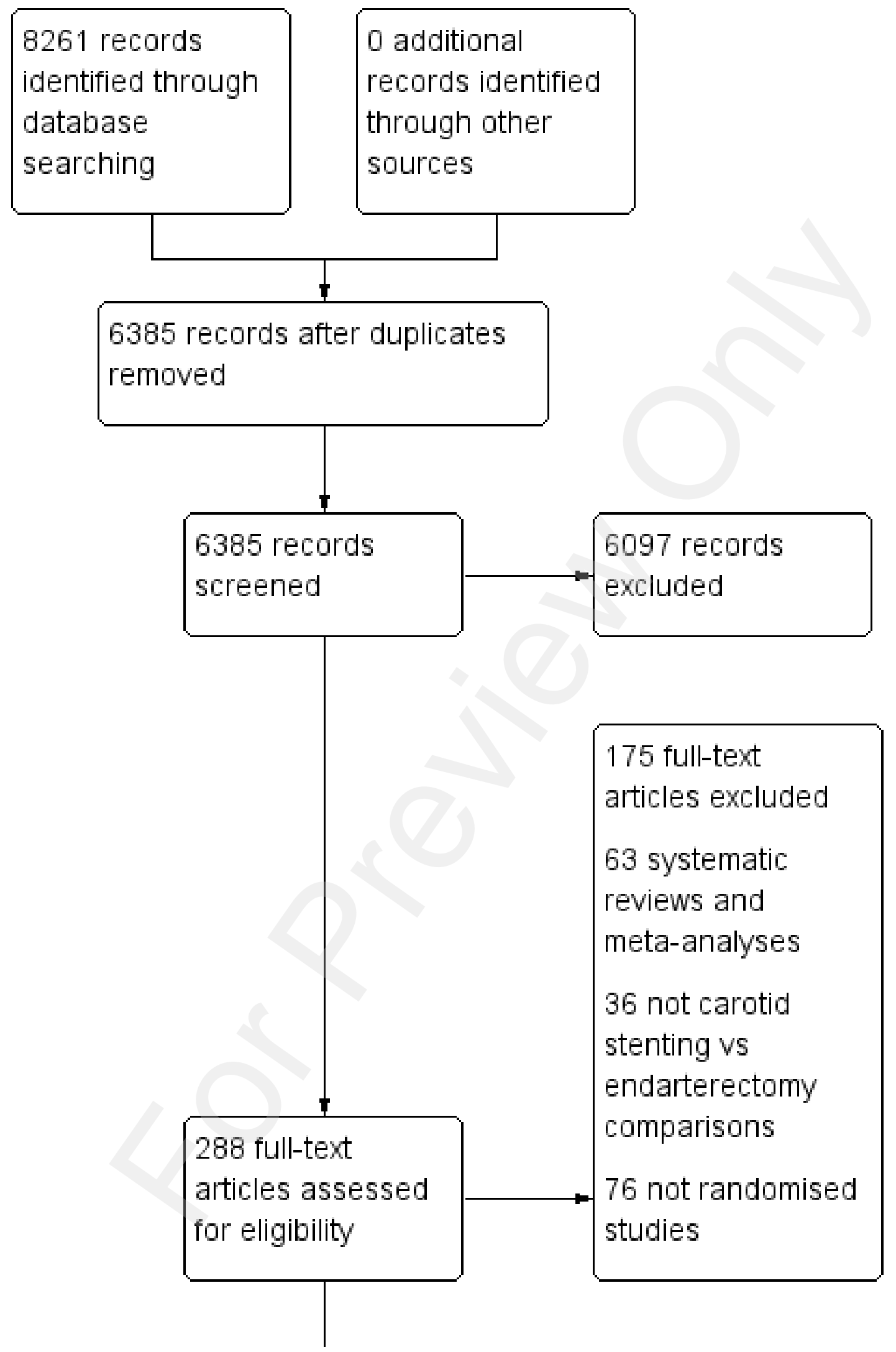


Figure 1. (Continued)

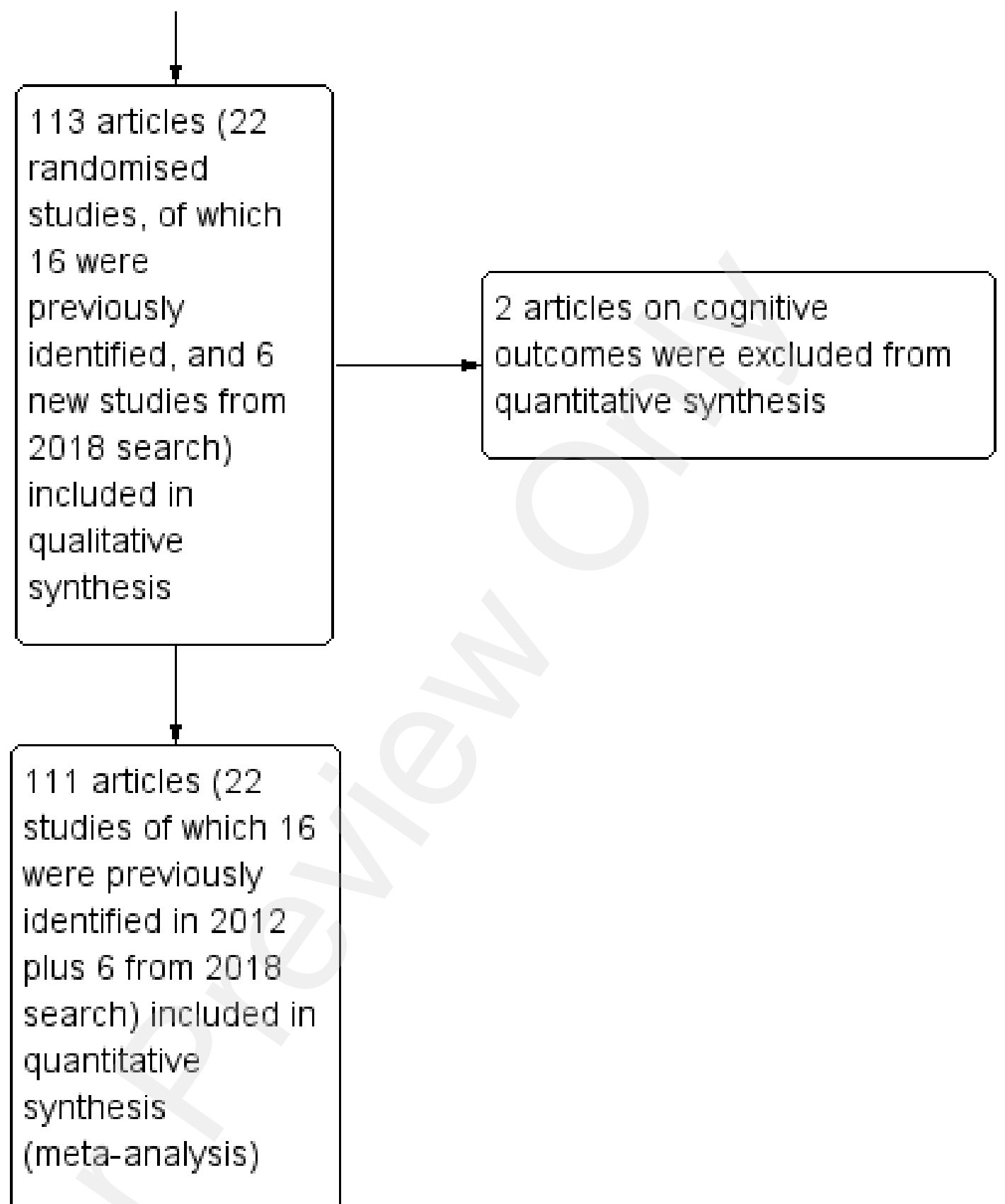

\section{Included studies}

\section{Design, sample size, setting, and participants}

One trial of stenting versus endarterectomy in participants with symptomatic carotid stenosis of $70 \%$ or greater was carried out at the Leicester Royal Infirmary, UK (a university teaching hospital) (Leicester 1998). The trial randomised 23 participants between June and September 1996, but was then stopped by the data monitoring committee for reasons of safety. The results were published in 1998. Four of 11 participants randomised to endovascular treatment and two of 12 participants randomised to endarterectomy did not receive their allocated treatment and were excluded from analysis in this trial.
One industry-funded multicentre randomised trial of stenting versus endarterectomy in the USA was stopped early by the sponsor for reasons of safety and futility after enrolling 219 participants with symptomatic stenosis of $60 \%$ or greater (WALLSTENT 2001). Results were presented at the 2001 International Stroke Conference and were only available in the form of an abstract for the present review. Numbers of participants with outcome events were derived from percentages provided in the abstract. Further results from this trial have not been published to date.

The Carotid And Vertebral Artery Transluminal Angioplasty Study (CAVATAS) was an international randomised multicentre study incorporating two separate trials of carotid stenosis and one trial of vertebral artery stenosis. In the first trial, 505 participants 
with symptomatic or asymptomatic carotid stenosis considered to require revascularisation and equally suitable for both procedures were randomised between endovascular treatment (252 participants) or endarterectomy (253 participants) between March 1992 and July 1997 (CAVATAS-CEA 2001). Ninety per cent of randomised participants had ischaemic symptoms associated with the randomised carotid artery in the six months prior to randomisation, and all had at least a moderate degree of stenosis. Initial results were published in 2001, and long-term results up to 11 years after randomisation were published in 2009. One participant was randomised to endovascular treatment in error and was excluded from the analysis. In 240 participants in the endovascular arm and 246 participants in the surgery arm treatment was initiated as randomly allocated.

In the second CAVATAS trial, 40 participants with symptomatic or asymptomatic carotid stenosis warranting treatment, but who were considered unsuitable for endarterectomy because of surgical or medical contraindications, were randomised between endovascular treatment plus best medical care (20 participants) or best medical care alone (20 participants) between April 1992 and May 1997 (CAVATAS-MED 2009). Results, including follow-up to 10 years after randomisation, were published in 2009. Treatment was initiated as randomly allocated in 16 participants in the combined arm and 19 participants in best medical care arm.

One single-centre trial at a community hospital (Central Baptist Hospital) in Lexington, KT, USA, randomised 104 participants with symptomatic carotid stenosis of greater than $70 \%$ between stenting (53 participants) or endarterectomy (51 participants) (Kentucky 2001). The same group conducted a single-centre trial comparing the two treatments in 85 participants with asymptomatic carotid stenosis of greater than $80 \%$ between stenting ( 43 participants) and endarterectomy (42 participants) (Kentucky 2004). In both trials, all randomised participants were treated and there was no mention of participants crossing over from the randomly allocated treatment to the alternative one. In 2014, long-term follow-up data were published for both trials combined. Outcome events according to symptom status were not consistently provided.

One single-centre trial conducted at Xuanwu Hospital in Beijing, China, randomised 21 participants with greater than $70 \%$ carotid stenosis on one side and contralateral occlusion who were apparently considered unsuitable for surgery - to endovascular treatment (eight participants) or medical care alone (13 participants), between 2001 and 2003 (Beijing 2003). Results were published in 2003.

Stenting and Angioplasty with Protection in Patients at High Risk for Endarterectomy (SAPPHIRE) was a multicentre randomised trial in the USA (SAPPHIRE 2004). From August 2000 to July 2002, 334 participants with $50 \%$ or greater symptomatic carotid stenosis or $80 \%$ or greater asymptomatic stenosis, who had at least one condition considered to potentially increase the risk of surgical complications, were randomised between endovascular treatment and endarterectomy (167 participants in each arm). The trial was terminated early because of a drop in recruitment numbers. One hundred and fifty-nine participants in the endovascular arm and 151 participants in the surgery arm received the allocated treatment. Results were published initially in 2004 and upon completion of long-term follow-up in 2008. Although cumulative incidences of the primary endpoint, a composite of periprocedural death, stroke, or myocardial infarction or death or ipsilateral stroke during follow-up, were reported for symptomatic and asymptomatic participants separately, numbers of separate outcome events according to symptom status were not provided.

One Chinese multicentre trial, also led by Xuanwu Hospital in Beijing, reported the results of 166 participants with severe symptomatic or asymptomatic carotid stenosis randomised between endovascular treatment (82 participants) and endarterectomy (84 participants) (TESCAS-C 2006). The original study was published in Chinese in 2006 with only the abstract available in English. We extracted data for the present review from the abstract and the website (www.trialresultscenter.org), but details of the trial were not available because we were unable to obtain a translation of the article.

One French multicentre randomised trial called Endarterectomy versus Angioplasty in Patients with Symptomatic Severe Carotid Stenosis (EVA-3S) was started in November 2000 and randomised participants with $60 \%$ or greater symptomatic carotid stenosis between endovascular treatment and endarterectomy (EVA-3S 2006). The trial was stopped early by the safety committee because of safety and futility concerns in September 2005, after 527 participants had been enrolled (endovascular treatment: 265 participants, endarterectomy: 262 participants). Two hundred and sixty participants in the endovascular arm and 257 participants in the endarterectomy arm received the randomly allocated treatment. Results up to six months after randomisation were published in 2006, and up to four years after randomisation in 2008. Results of long-term follow-up of a median of 7.1 years were published in 2014.

The Stent-supported Percutaneous Angioplasty of the Carotid artery versus Endarterectomy (SPACE) trial randomised 1214 participants with symptomatic carotid stenosis of $50 \%$ or greater or $70 \%$ or greater (depending on the method of measurement) between endovascular treatment ( 613 participants) or endarterectomy (601 participants) in Germany, Austria, and Switzerland, from March 2001 to February 2006 (SPACE 2006). Following an interim analysis, the trial was stopped by the steering committee for reasons of futility and lack of funding. The randomly allocated treatment was initiated in 591 participants in the endovascular arm and 567 participants in the endarterectomy arms. Short-term outcomes were published in 2006, and results up to two years after randomisation in 2008.

One single-centre substudy of SPACE evaluated cognitive outcomes after endarterectomy versus endovascular treatment in participants with symptomatic carotid stenosis. The substudy enrolled 48 participants, of whom 24 were assigned to endarterectomy and 21 to endovascular treatment. Three participants were lost to follow-up. Neuropsychological follow-up was completed in 45 participants.

In 2009, the Stent-supported Percutaneous Angioplasty of the Carotid artery versus Endarterectomy trial 2 (SPACE-2) began recruiting participants. Initially the trial was planned as a three-armed, randomised controlled trial comparing best medical treatment (BMT) alone to endarterectomy plus BMT or endovascular therapy plus BMT (SPACE-2 2016). Due to slow enrolment the study design was amended in 2013 to become two parallel randomised trials, one comparing BMT alone to endarterectomy and the second comparing BMT to endovascular therapy. This change in study design did not lead to an increase 
in participant recruitment and the trial was stopped early after inclusion of 513 participants over a five-year period. Outcomes within the procedural time period of the recruited participants were reported in 2016.

One single-centre trial conducted at the University Hospital Basel, Switzerland (Basel Carotid Artery Stenting Study - BACASS) randomised 20 participants with symptomatic carotid stenosis of $70 \%$ or greater to endovascular treatment (10 participants) or endarterectomy (10 participants) between November 1998 and February 2002 (BACASS 2008). Recruitment was stopped when the centre started randomising participants in ICSS 2010, and results, including long-term follow-up over a median of four years after treatment, were published in 2008. All participants received the randomly allocated treatment.

One single-centre trial was started in August 1999 at the University Hospital Regensburg, Germany, enrolling participants with $70 \%$ or greater symptomatic carotid stenosis (Regensburg 2008). The trial was stopped in April 2002 after randomisation of 43 participants to endovascular treatment and 44 participants to endarterectomy when the multicentre SPACE 2006 trial, which had a similar design, started. All randomised participants were treated. The paper did not specify whether the applied treatment was always the one randomly allocated, but did not report any cross-overs.

Another single-centre trial conducted in China randomised 40 participants with symptomatic carotid stenosis of $50 \%$ or greater or asymptomatic stenosis of $70 \%$ or greater between carotid stenting or endarterectomy from May 2004 and December 2006 (20 participants undergoing 23 procedures in each arm - three participants in each arm had bilateral disease) (Beijing 2009). The original study was published in Chinese in 2009 with only the abstract available in English, which we used to extract data. We were unable to obtain a translation of the article.

The ICSS randomised 1713 participants with symptomatic carotid stenosis of $50 \%$ or greater to carotid stenting (855 participants) or endarterectomy (858 participants) between May 2001 and October 2008 in Europe, Australia, New Zealand, and Canada. Shortterm results up to 120 days after randomisation were published in 2010 (ICSS 2010). The randomised procedure was initiated in 828 in the carotid stenting arm and 821 participants in the endarterectomy arm. Long-term follow-up in this trial ended in 2011 and the results were published in 2014. A substudy evaluating cognitive performance after carotid endarterectomy versus endovascular treatment enrolled 177 participants, of whom 140 had neuropsychological evaluation before treatment and 120 participants at follow-up.

The Carotid Revascularization Endarterectomy versus Stenting Trial (CREST) was a multicentre randomised trial conducted in the USA and Canada (CREST 2010). Between December 2000 and July 2008, 2522 participants with carotid stenosis were randomly assigned to carotid stenting (1271 participants) or endarterectomy (1251 participants). The trial initially enrolled only people with symptomatic carotid stenosis, but the eligibility criteria were changed in 2005 to also include people with asymptomatic stenosis. The final population consisted of 1321 participants with symptomatic and 1181 participants with asymptomatic stenosis. Results up to four years after randomisation were published in 2010. The randomly assigned treatment was initiated in 1152 participants in the carotid stenting arm and 1194 participants in the endarterectomy arm. Results over 10 years of follow-up were published in 2016.

One single-centre trial conducted in the Czech Republic randomised 150 participants with asymptomatic or symptomatic carotid stenosis of $70 \%$ or greater to carotid stenting with a cerebral protection device (77 participants) or endarterectomy (73 participants) between October 2010 and July 2014 (Ostrava 2014). All participants received the randomly allocated treatment. Results were published in 2014.

The Randomized Trial of Stent versus Surgery for Asymptomatic Carotid Stenosis (ACT-1) was a multicentre randomised controlled trial conducted in the USA (ACT-1 2016). Between April 2005 and March 2013, 1453 participants with asymptomatic carotid stenosis of greater than $70 \%$ were randomly assigned in a 3:1 ratio to carotid stenting (1089 participants) or endarterectomy (364 participants). The initially planned sample size was 1658 participants, but the study was stopped prematurely due to slow enrolment.

One multicentre study conducted in China randomised 63 participants considered at high surgical risk with asymptomatic carotid stenosis of $70 \%$ or greater or symptomatic carotid stenosis of greater than $50 \%$ to either carotid stenting (28 participants) or endarterectomy (35 participants) (Beijing 2013). All randomised participants were treated. The paper did not specify whether the applied treatment was always the one randomly allocated, but did not report any cross-overs.

One single-centre study evaluated cognitive performance after endarterectomy versus endovascular treatment in participants with asymptomatic carotid stenosis greater than $80 \%$ (Houston 2014). This study enrolled 60 participants, 29 of whom were allocated to carotid stenting and 31 to endarterectomy. The investigators reported one cross-over from carotid stenting to endarterectomy. Two participants withdrew from the study after treatment (one participant in each treatment group) and three participants (two in the endarterectomy group and one in the carotid stenting group) were lost to follow-up.

One single-centre study conducted in Israel randomised 136 participants with asymptomatic severe (greater than $70 \%$ ) carotid stenosis in a 1:1 ratio to either endarterectomy (68 participants) or carotid artery stenting (68 participants) (Carmel Medical Center 2017). Three participants were lost to follow-up and one participant crossed over from endovascular treatment to endarterectomy.

\section{Endovascular treatment}

Details of carotid stenting and endarterectomy in the included trials are provided in the Characteristics of included studies tables. While in earlier trials balloon angioplasty with or without the insertion of a stent device was performed (Leicester 1998; CAVATAS-CEA 2001; CAVATAS-MED 2009), all other trials performed primary stenting of the carotid artery with various devices and protection systems.

In the CAVATAS-CEA 2001 trial, all participants enrolled in the endovascular arm before 1994 received percutaneous balloon angioplasty without insertion of stents. Stents suitable for the carotid artery became available during the course of the study. From 1994, stenting was allowed at the discretion of the intervening radiologist. Stents were used in 55 participants $(22 \%$ of those allocated endovascular treatment), usually after unsatisfactory results with prior balloon angioplasty. In all the other trials, stents 
were routinely used in the endovascular arms, alone or in addition to balloon angioplasty.

There were also differences in the use of cerebral protection devices during endovascular treatment among the included trials. Protection devices were either not yet available or were not used in the Leicester 1998, CAVATAS-CEA 2001, Kentucky 2001, WALLSTENT 2001, Kentucky 2004, Regensburg 2008, and CAVATASMED 2009 trials. Use of protection devices was mandatory or part of routine practice in the SAPPHIRE 2004, BACASS 2008, Beijing 2009, CREST 2010, Beijing 2013, and ACT-1 2016 trials. The EVA-3S 2006 trial briefly interrupted randomisation in 2003 and made protection device use compulsory following an interim analysis; protection devices were used in $227(87 \%)$ of all 260 stent procedures initiated as randomly allocated (per protocol). In the SPACE 2006 trial, protection devices were optional and used in 154/591 (26\%) per-protocol stent procedures. In ICSS 2010, deployment of a protection device was recommended, wherever it was considered safe and feasible; protection devices were used in 593/828 (72\%) per-protocol stent procedures. In SPACE-2 2016, the use of cerebral protection devices was left to the discretion of the interventionist and no data were available on the use of these devices. In Ostrava 2014, cerebral protection devices were used in all but three participants (96\%). No information on protection device use was available from the Beijing 2009 and TESCAS-C 2006 trials.

A consultant radiologist, who had performed eight successful stent procedures in the carotid artery before the trial, performed all endovascular procedures in the Leicester 1998 trial. There were differences among the large multicentre trials in the amount of pretrial experience in endovascular treatment required for a centre to join. In the WALLSTENT 2001 trial, interventional physicians needed to have performed at least 10 carotid stent procedures. In the CAVATAS-CEA 2001 and CAVATAS-MED 2009 trials, prior expertise in endovascular treatment was required, albeit not in the carotid artery, as this was considered an entirely new treatment for carotid stenosis at the beginning of the trial. In the SAPPHIRE 2004 trial, interventionists had performed between 20 and 700 carotid stent procedures in total before the trial. Interventional physicians in the EVA-3S 2006 trial had to have performed at least 12 carotid stenting procedures or at least 35 stenting procedures of the supra-aortic trunks including five carotid stents, before joining the trial. Less-experienced interventionists were still allowed to join but had to perform all procedures under supervision until sufficient numbers had been achieved. Of 260 per-protocol stent procedures, 101 (39\%) were carried out under supervision. In the SPACE 2006 trial, interventionists had to show proof of at least 25 successful consecutive percutaneous transluminal angioplasty or stent procedures in the carotid artery. In ICSS 2010, interventional physicians were required to have carried out at least 50 stent procedures, 10 of which in the carotid artery. Centres with less experience (so-called supervised centres) were allowed to join the trial under the condition that procedures were supervised by an experienced interventionist. Of all 855 participants randomised in the stent arm, $102(12 \%)$ were enrolled at supervised centres. In the CREST 2010 trial, interventionists with fewer than 30 carotid stent procedures were required to complete a specific training programme. Interventionists' eligibility to participate was based on evaluation of their performance during a mean of 20 procedures in a non-randomised lead-in phase to the trial. In SPACE-2 2016, the interventional physician needed to have performed at least
40 procedures within the last two years and participate in quality assessment. In ACT-1 2016, the interventionist had to be approved by the sponsor. However, details about the required level of experience were not provided.

Among the singe-centre studies, interventionists had prior experience of eight carotid stent procedures in the Leicester 1998 trial, and at least 15 carotid stent procedures in the BACASS 2008 trial. In Ostrava 2014, the centre had performed more than 500 carotid artery stenting procedures in the past five years. Detailed numbers of pretrial experience in endovascular treatment were unknown for the Kentucky 2001, Beijing 2003, Kentucky 2004, Regensburg 2008, Beijing 2009, and Beijing 2013 trials.

\section{Follow-up}

Length of follow-up and frequency of follow-up visits varied between trials. All trials, except one (Beijing 2003), prespecified a clinical assessment for periprocedural complications one month or 30 days after treatment. Most trials also included a clinical assessment soon after the procedure, ranging from one to seven days after treatment (Leicester 1998; Kentucky 2001; WALLSTENT 2001; Kentucky 2004; SAPPHIRE 2004; EVA-3S 2006; SPACE 2006; BACASS 2008; Regensburg 2008; CREST 2010; Ostrava 2014).

Prospective follow-up was continued beyond one month in all trials except two (Leicester 1998; Ostrava 2014), and went up to six months (TESCAS-C 2006; Beijing 2013; Houston 2014), 12 months (WALLSTENT 2001; Regensburg 2008; Beijing 2013), 18 months (Beijing 2003; Beijing 2009), two years (SPACE 2006), three years SAPPHIRE 2004), five years ( ACT-1 2016; Carmel Medical Center 2017), 10 years or longer (ICSS 2010, median 4.2 years; EVA-3S 2006, median 7.1 years; CREST 2010, median 7.4 years; Kentucky 2001 and Kentucky 2004, minimum follow-up 10 years) or without predefined maximum length of follow-up (CAVATAS-CEA 2001, median five years; CAVATAS-MED 2009, median 4.5 years in the endovascular arm and 3.5 years in the medical arm; BACASS 2008, mean 48 months in the endovascular arm and 44 months in the surgical arm). In its final publication, the Regensburg 2008 trial included results of a retrospective extension of follow-up with a median of 66 months in the endovascular arm and 64 months in the surgical arm. In SPACE-2 2016, no follow-up data beyond the procedural period were available.

\section{Outcome measures}

The definition of stroke specified for the present review was also used in most trials which provided their definitions (Leicester 1998; SAPPHIRE 2004; SPACE 2006; BACASS 2008; Regensburg 2008; CREST 2010; ICSS 2010; ACT-1 2016; SPACE-2 2016). The stroke definition used in the CAVATAS-CEA 2001 trial initially included only events with symptoms lasting more than seven days. However, in the final analysis including long-term follow-up, periprocedural strokes lasting seven days or less were counted as well (beyond 30 days after treatment, all recorded stroke outcome events lasted longer than seven days). These events were also included in the present review. Where definitions of stroke were unavailable, the review authors assumed that the same definition as in the present review was used.

Most trials classified stroke severity according to functional disability; this was defined by a score of 3 or more on the mRS (CAVATAS-CEA 2001; EVA-3S 2006; SPACE 2006; CAVATAS-MED 2009; ICSS 2010; ACT-1 2016; SPACE-2 2016), or on the Oxfordshire 
Handicap Stroke score (Leicester 1998). In EVA-3S 2006, an increase in level of disability over the baseline level of at least two points on the $\mathrm{mRS}$ was required in addition; a single event was reclassified by the EVA-3S investigators for a previous pooled analysis omitting the additional criterion (CSTC 2010), and numbers of disabling strokes were extracted accordingly.

Four trials classified strokes as minor or major. In the SAPPHIRE 2004 trial, this was done based on the NIHSS, the Barthel Index, and the Rankin Scale, but cut-off levels on these scales to classify a stroke as major were not provided. In BACASS 2008 an increase in NIHSS score by 4 or more points, or symptoms including aphasia or hemianopia lasting for more than 24 hours were required for major stroke; one single stroke occurred fulfilling this definition, but the study authors stated that 30 days after stroke onset the mRS was 1 . Thus, the event was not included as a major or disabling stroke in the present review. The definition for major stroke used in CREST 2010 included a score on the NIHSS of 9 or more, 90 days after the procedure, or was made "on the basis of clinical data," without further specification. In ACT-1 2016 major stroke was defined as either an increase in the NIHSS of more than 4 points from the prestroke score or an increase in the Rankin Scale score of more than 2 points from the prestroke score or any stroke which led to a Rankin Scale score of 5 or more.

The WALLSTENT 2001 and Regensburg 2008 trials used a combination of Barthel Index, Rankin Scale or mRS, and NIHSS, and the Kentucky 2001 trial used a combination of Barthel Index and $\mathrm{mRS}$ to assess functional outcome. Functional assessment was not specified in the remaining trials (Beijing 2003; Kentucky 2004; TESCAS-C 2006; Beijing 2009; Beijing 2013; Ostrava 2014). Numbers of disabling or major strokes were not available from those trials, although no strokes were reported to have occurred in the Kentucky 2001 and Kentucky 2004 trials.

The protocol of one trial explicitly stated that visual loss lasting longer than 24 hours which resulted from retinal ischaemia (i.e. retinal stroke) was included in the outcome definition of stroke (ICSS 2010); correspondence with investigators from two other trials also confirmed that retinal strokes were included (EVA-3S 2006; SPACE 2006). Retinal strokes were assessed but initially excluded from reports of the CAVATAS-CEA 2001 trial; they were included in the definition of stroke for the present review.

Trials also differed in assessment and definitions of myocardial infarction. Among trials that reported myocardial infarction as an endpoint, two used the World Health Organization (WHO) definition (EVA-3S 2006; ICSS 2010), which includes two out of the following three criteria: prolonged typical chest pain, elevation of specific cardiac enzymes more than twice the upper limit of normal, and specific electrocardiogram (ECG) abnormalities. In SAPPHIRE 2004, myocardial infarction was defined solely on the basis of a creatine kinase elevation higher than two times the upper limit of normal with a positive MB fraction. This implied that enzymes were routinely measured after treatment. The trial provided the numbers of participants with Q-wave infarction separately, but in the composite outcome measures of which myocardial infarction was part, all types were included. In the CREST 2010 trial, participants were routinely screened with ECG and cardiac enzyme measurement before and after treatment. Myocardial infarction was defined by a creatine kinase MB (CK$M B$ ) or troponin level that was twice the upper limit of normal plus either chest pain or symptoms consistent with ischaemia, or ECG evidence of ischaemia. In ACT-1 2016, participants were also routinely screened with ECG and measurement of cardiac enzymes before and after treatment. Myocardial infarction was defined as an elevation and gradual fall of troponin or CK-MB plus at least one of the following criteria: symptoms of ischaemia, development of pathological Q waves on ECG or ECG changes indicative of cardiac ischaemia. In Ostrava 2014, participants were also routinely screened with ECG and clinical examination before and after treatment. Myocardial infarction was defined as a greater than two-fold increase in cardiac troponin T level or ECG evidence of ischaemia. In the Carmel Medical Center 2017 trial, myocardial infarction was defined as relevant clinical symptoms and elevated cardiac enzymes.

The SPACE 2006 trial did not include myocardial infarction as a predefined endpoint, but for the purpose of a previous pooled analysis, the investigators reassessed all adverse event reports and found no reported myocardial infarctions in the periprocedural period fulfilling WHO criteria (CSTC 2010). Two trials reported numbers of myocardial infarctions without providing definitions (CAVATAS-CEA 2001; BACASS 2008). The Kentucky 2001 trial reported that one participant died of an myocardial infarction immediately after endarterectomy but did not specify inclusion of non-fatal myocardial infarction as an outcome measure; therefore, this trial was excluded from the comparison of all myocardial infarction in this review. No information on myocardial infarction was available from the remaining trials.

Twelve trials reported cranial nerve palsies of any severity (Leicester 1998; CAVATAS-CEA 2001; Kentucky 2001; Kentucky 2004; SAPPHIRE 2004; EVA-3S 2006; BACASS 2008; Regensburg 2008; CREST 2010; ICSS 2010; Beijing 2013; ACT-1 2016). In the CREST 2010 trial, only cranial nerve palsies not resolving for at least one month after treatment were counted. Five trials reported numbers of participants with access site haematoma requiring surgery, blood transfusion, or prolonging hospital stay (CAVATAS-CEA 2001; Kentucky 2001; Kentucky 2004; EVA-3S 2006; ICSS 2010). We also included access site haematomas reported in the Regensburg 2008, Beijing 2009, and ACT-1 2016 trials in the present review, although no definition of severity was provided. The SPACE 2006 trial did not report on cranial nerve palsy and haematoma initially, but adverse event reports were reassessed for the aforementioned pooled analysis (CSTC 2010), and numbers of participants with these outcomes were extracted for the present review. The CREST 2010 trial reported several types of bleeding complications including surgical wound complications requiring treatment, which was used in the present review.

Fourteen trials specified that carotid ultrasound was performed at regular intervals during follow-up to detect restenosis (CAVATASCEA 2001; Kentucky 2001; WALLSTENT 2001; Kentucky 2004; SAPPHIRE 2004; EVA-3S 2006; SPACE 2006; CAVATAS-MED 2009; BACASS 2008; Regensburg 2008; CREST 2010; ICSS 2010; Beijing 2013; Carmel Medical Center 2017). The Beijing 2003 trial carried out a single ultrasound examination to assess restenosis 1.5 years after treatment. In addition, the TESCAS-C 2006 and Beijing 2009 trials specified restenosis as a secondary outcome in the English abstracts, albeit without any information how this was assessed. Beijing 2013 also specified severe restenosis assessed by carotid ultrasound as a secondary endpoint. Participants in this study were followed with ultrasound at one month, six months, and 12 months. Twelve trials comparing endovascular treatment with 
endarterectomy reported numbers of participants with restenosis (CAVATAS-CEA 2001; Kentucky 2001; Kentucky 2004; EVA-3S 2006; SPACE 2006; BACASS 2008; Regensburg 2008; Beijing 2009; CREST 2010; ICSS 2010; Beijing 2013; Carmel Medical Center 2017). No data on restenosis have been published from the remaining trials to date.

In addition, three trials reported randomised evidence on endarterectomy versus stenting for prevention of cognitive decline in participants with extracranial carotid stenosis. One trial included only participants with asymptomatic carotid stenosis (Houston 2014), while two were substudies of larger trials including participants with symptomatic carotid stenosis (SPACE 2006; ICSS 2010). In Houston 2014, 60 participants were randomly allocated to carotid artery stenosis (29 participants) or endarterectomy (31 participants) and assessed with multiple tests on cognitive domains of attention, memory, mood, visual-spatial skills, motor ability, processing speed, and executive functioning 10 days or fewer before treatment as well as six weeks and six months after treatment. Tests were administered using standardised conditions and scored by raters who were blinded to treatment allocation. In the SPACE 2006 substudy, 48 participants were randomly allocated to carotid artery stenting or endarterectomy, but only 45 participants (carotid artery stenting: 21 participants; endarterectomy: 24 participants) completed follow-up and were assessed with multiple tests on cognitive domains of memory, attention, executive function, and motor skills before treatment, as well as six days and 30 days after treatment. In ICSS 2010, a subset of 177 participants (carotid artery stenting: 90 participants; endarterectomy: 87 participants) were enrolled in a substudy in which neuropsychological evaluation was performed before and six months after carotid revascularisation.

\section{Analysis of data}

Nine trials specified that the primary analysis followed the intention-to-treat principle as defined under Data collection and analysis (CAVATAS-CEA 2001; SAPPHIRE 2004; SPACE 2006; CAVATAS-MED 2009; CREST 2010; ICSS 2010; ACT-1 2016; SPACE-2 2016; Carmel Medical Center 2017). One trial used a modified intention-to-treat approach for analysis of periprocedural outcomes, excluding participants who received neither endovascular nor surgical treatment (EVA-3S 2006). A per-protocol analysis was carried out in the Leicester 1998 trial, excluding participants who did not receive the randomly allocated treatment. In the BACASS 2008 trial, no statistical analysis was performed due to the small number of participants enrolled, but it was stated that all participants received the randomly allocated treatment. Four further trials stated that all randomised participants received treatment, but it was not specified whether this was always the randomly allocated treatment (Kentucky 2001; Kentucky 2004; Regensburg 2008; Ostrava 2014); however, no mention of participants crossing over to the alternative treatment was made in these trials. Numbers of participants receiving treatment were unknown in the other five trials (WALLSTENT 2001; Beijing 2003; TESCAS-C 2006; Beijing 2009; Beijing 2013).

Four trials specified a non-inferiority hypothesis in which stenting was considered as non-inferior to endarterectomy when the $90 \%$ or $95 \% \mathrm{Cl}$ of the absolute difference in the risk of the primary outcome measure with stenting minus the risk of the primary outcome measure with surgery was below a predefined margin (SAPPHIRE 2004; EVA-3S 2006; SPACE 2006; ACT-1 2016).
SPACE-2 was planned as a three-arm randomised controlled trial which aimed to show superiority of intervention (carotid artery stenting or endarterectomy) compared to conservative treatment alone and in a second step, in case of superiority of both interventions, testing of a non-inferiority hypothesis in which stenting was considered as non-inferior to endarterectomy was planned.

For the analysis of treatment safety, most trials defined the periprocedural period as the first 30 days after treatment (Leicester 1998; CAVATAS-CEA 2001; Kentucky 2001; WALLSTENT 2001; Kentucky 2004; SAPPHIRE 2004; EVA-3S 2006; TESCAS-C 2006; BACASS 2008; Beijing 2009; CAVATAS-MED 2009; ACT-1 2016; SPACE-2 2016). Among those, two trials reported events occurring between randomisation and treatment and excluded those from analysis of periprocedural outcomes (CAVATAS-CEA 2001; EVA-3S 2006). One trial reported that some participants did not receive the allocated treatment because of "deterioration of their condition," but it was unclear whether this was a consequence of prespecified endpoints such as stroke or myocardial infarction (SAPPHIRE 2004). In four trials, the observation period for analysis of short-term outcome started at randomisation, and lasted up to 30 days after treatment, or 30 days after randomisation in participants receiving no revascularisation (SPACE 2006; Ostrava 2014); up to 30 days after treatment or 36 days after randomisation in participants receiving no revascularisation (CREST 2010); or up to 120 days after randomisation in all participants (ICSS 2010).

The Regensburg 2008 trial separately reported outcome events occurring during the prospectively defined 12-month follow-up period and the retrospective extension of follow-up, but without specifying how many events occurred within 30 days of treatment. The review authors assumed that the reported myocardial infarction, cranial nerve palsy, and access site haematoma occurred periprocedurally; however, we excluded the trial from comparisons involving periprocedural stroke.

Three trials reported results on cognition after carotid endarterectomy versus carotid stenting (SPACE 2006; ICSS 2010; Houston 2014). In the substudy of ICSS 2010, endovascular therapy was associated with a larger decrease in cognition than endarterectomy although the difference did not reach statistical significance. In the SPACE 2006 substudy, there was no significant difference in the cognitive performance between the two treatment groups. In Houston 2014, verbal and visual memory and attention functions substantially improved after endarterectomy and endovascular therapy compared to baseline. In addition, endovascular treatment was associated with statistically significant improvements in cognitive processing speed at six weeks, as well as executive functioning and motor function at six months.

Due to substantial heterogeneity in the tests used to assess cognitive performance as well as differences in time points at which cognitive performance after treatment was assessed in these trials, meta-analysis of the results was not possible.

\section{Ongoing and planned studies}

We are aware of three ongoing randomised trials investigating treatment of asymptomatic carotid stenosis: one large ongoing multicentre trial, the Asymptomatic Carotid Surgery Trial 2 (ACST-2), randomises participants between stenting or 
endarterectomy (ACST-2); one large ongoing multicentre trial, the Carotid Revascularization and Medical Management for Asymptomatic Carotid Stenosis Trial 2 (CREST-2), consisting of two independent trials randomising participants between stenting with embolic protection versus BMT alone or between endarterectomy versus BMT alone (CREST-2); another ongoing multicentre trial randomises participants with asymptomatic carotid stenosis at higher-than-average risk of ipsilateral stroke identified by ancillary investigations (presence of transcranial Doppler (TCD) detected embolic signals, intraplaque haemorrhage on MRI, TCD-measured impaired cerebral vasomotor reserve or rapid stenosis progression) to carotid endarterectomy combined with optimal medical therapy versus optimal medical therapy alone (NCT02841098: Endarterectomy Combined With Optimal Medical Therapy (OMT) vs OMT Alone in Patients with Asymptomatic Severe Atherosclerotic Carotid Artery Stenosis at Higher-than-average Risk of Ipsilateral Stroke).

A further trial, the Second European Carotid Surgery Trial (ECST-2), which is currently recruiting participants, compares revascularisation by endarterectomy or stenting combined with optimal medical therapy versus optimal medical therapy alone in people with asymptomatic or low- to intermediate-risk symptomatic carotid stenosis. A specific carotid artery risk score identifies people eligible to participate.

\section{Excluded studies}

We excluded a total of 175 studies. Sixty-three studies were excluded because they were systematic reviews or meta-analyses, 36 were excluded because they did not entail direct comparisons of carotid endarterectomy versus carotid artery stenting, and 76 studies were excluded because they were not randomised.

\section{Risk of bias in included studies}

\section{Allocation}

All included trials stated that allocation of treatment was randomised. The method of randomisation was given for 16 trials. In the large multicentre trials, central randomisation services were accessed by telephone, fax, or automated telephone response systems (CAVATAS-CEA 2001; SAPPHIRE 2004; EVA-3S 2006; SPACE 2006; CAVATAS-MED 2009; CREST 2010; ICSS 2010; Houston 2014). Some trials specified that the random allocation sequence was generated with a computer pseudo-random number generator (WALLSTENT 2001; SAPPHIRE 2004; EVA-3S 2006; SPACE 2006; Ostrava 2014; ACT-1 2016; SPACE-2 2016). In the WALLSTENT 2001 trial, each centre received its own randomisation sequence and treatment was assigned in sequentially numbered sealed envelopes. Among the single-centre studies, two trials used sequentially numbered sealed envelopes with random treatment assignments (Leicester 1998: 300 envelopes; BACASS 2008: 50 envelopes), while treatment assignment was performed in two other trials using a "random key" (Regensburg 2008), or a random table (Beijing 2003). For these 16 trials, we judged random sequence generation and allocation concealment to be adequate and the risk of selection bias low. We were unable to obtain detailed information regarding method of randomisation from the authors of six trials in which we judged the risk of selection bias to be unclear (Kentucky 2001; Kentucky 2004; TESCAS-C 2006; Beijing 2009; Beijing 2013; Carmel Medical Center 2017).
Restricted randomisation was reported in some trials, by use of a minimisation algorithm, taking account of centre and timing of symptoms (CAVATAS-CEA 2001; CAVATAS-MED 2009); or sex, age, contralateral carotid occlusion and side of the randomised carotid stenosis (ICSS 2010), or by use of randomly permuted blocks, stratified by centre and degree of stenosis (EVA-3S 2006), or by centre and symptomatic status (CREST 2010). The Regensburg 2008 trial stated that randomisation was stratified according to age, degree of stenosis, and presence of coronary heart disease, without detailing the method of restriction.

\section{Blinding}

Due to study design and the nature of the interventions, participants, health workers, and assessors were not blinded to treatment or outcome in any of the trials included in this review. Moreover, participants treated by carotid endarterectomy were often managed postoperatively on surgical wards where minor neurological events may be missed or misinterpreted. Analyses restricted to disabling stroke or death were assumed to be the least affected by this bias. We excluded transient ischaemic attacks from the analysis because they are likely to be the most affected by this bias.

Nonetheless, in most trials, participants were followed-up by neurologists or clinicians who were not directly involved in the procedures (Leicester 1998; CAVATAS-CEA 2001; Kentucky 2001; WALLSTENT 2001; Kentucky 2004; SAPPHIRE 2004; EVA-3S 2006; SPACE 2006; CAVATAS-MED 2009; BACASS 2008; Regensburg 2008; CREST 2010; ICSS 2010; Houston 2014; Ostrava 2014; ACT-1 2016; SPACE-2 2016); in addition, the large multicentre trials adjudicated outcome events centrally, blinded to treatment (CAVATAS-CEA 2001; SAPPHIRE 2004; EVA-3S 2006; SPACE 2006; CAVATAS-MED 2009; CREST 2010; ICSS 2010). In those trials, we considered the risk of detection bias to be low. In five trials, there was no information on independent follow-up or blinded adjudication and we considered the risk of detection bias to be unclear (Beijing 2003; TESCAS-C 2006; Beijing 2009; Beijing 2013; Carmel Medical Center 2017).

\section{Incomplete outcome data}

In five trials, some participants were randomised but later excluded from analysis: in CAVATAS-CEA 2001, one participant was shown to have carotid occlusion after randomisation; 18 participants randomised in SPACE 2006, and three participants randomised in ICSS 2010 immediately withdrew consent after randomisation; and 20 participants randomised in CREST 2010 at a single centre were excluded owing to scientific misconduct. We considered the risk of attrition bias arising from these exclusions to be small. In the Leicester 1998 trial, 4/11 participants randomised to endovascular treatment and 2/12 participants randomised to surgery did not receive their allocated treatment (see Characteristics of included studies tables for reasons) and were excluded from analysis in this trial. Outcome events occurring after randomisation were not systematically reported for these participants and it was unclear whether the participants refusing the allocated treatment crossed over to receive the alternative treatment. Therefore, we considered risk of attrition bias in this trial to be unclear. The participants excluded from analysis in those five trials were also excluded from the present review as no outcome data were available from them.

Most trials that followed up participants beyond 30 days after treatment provided the numbers of participants at risk for the primary outcome measure at various time points during follow- 
up or numbers of participants with available long-term followup, or both (ACT-1 2016; BACASS 2008; Beijing 2013; CAVATAS-CEA 2001; CAVATAS-MED 2009; CREST 2010; EVA-3S 2006; Regensburg 2008; SAPPHIRE 2004; SPACE 2006): details are provided in the 'Risk of bias' tables in the Characteristics of included studies table. In general, we assumed that censoring in these trials was noninformative, that is, unbiased in the sense that treatment effects were assumed to be the same in participants censored before the end of scheduled follow-up and those with complete followup (Higgins 2011). The Beijing 2003 trial reported that at 1.5 years' follow-up, the participants without outcome events "had not changed." The risk of attrition bias resulting from incomplete follow-up in these trials was considered low.

Leicester 1998, Beijing 2013, and SPACE-2 2016 did not provide details on excluded participants or on the exact number of participants at risk during follow-up. For these reasons the risk of attrition bias was deemed unclear (for details see Risk of bias in included studies).

From the remaining trials, neither numbers of participants at risk during follow-up nor numbers of participants with available longterm follow-up were provided, and we deemed the risk of attrition bias to be unclear (Kentucky 2001; WALLSTENT 2001; Kentucky 2004; TESCAS-C 2006; Beijing 2009).

\section{Selective reporting}

Most of the larger trials reported the primary outcome analysis as defined before in a separately published study protocol (CAVATAS CEA 2001; WALLSTENT 2001; EVA-3S 2006; SPACE 2006; CREST 2010; ICSS 2010; ACT-1 2016; SPACE-2 2016). Other trials made no references to study protocols, but reported major outcome events (i.e. strokes and deaths) as expected in general (Leicester 1998; Kentucky 2001; WALLSTENT 2001; Beijing 2003; Kentucky 2004; TESCAS-C 2006; BACASS 2008; CAVATAS-MED 2009; Beijing 2013; Ostrava 2014; Houston 2014; Carmel Medical Center 2017). In the Regensburg 2008 study, the primary outcome measure was specified to include events up to 30 days after treatment, but the final publication did not include a comparison of periprocedural events. However, we considered the risk of reporting bias in all those trials to be low.

The SAPPHIRE 2004 trial cited a protocol, albeit one that was published only a few months before the initial results of the trial. Expected major outcome events were reported, but not for symptomatic and asymptomatic participants separately. We could not exclude the possibility of reporting bias for this trial. In the Beijing 2013 trial, major outcome events were reported, but not for symptomatic and asymptomatic participants separately.

\section{Effects of interventions}

See: Summary of findings for the main comparison Carotid artery stenting compared to endarterectomy for symptomatic carotid stenosis; Summary of findings $\mathbf{2}$ Carotid artery stenting compared to endarterectomy for asymptomatic carotid stenosis; Summary of findings $\mathbf{3}$ Carotid artery stenting compared to endarterectomy for prevention of severe restenosis in people with symptomatic or asymptomatic carotid stenosis

We provide detailed results in the Data and analyses section.

\section{Comparison 1: stenting or endarterectomy for symptomatic carotid stenosis at standard surgical risk}

Seven trials compared carotid stenting with endarterectomy in participants with symptomatic carotid stenosis (Leicester 1998; Kentucky 2001; WALLSTENT 2001; EVA-3S 2006; SPACE 2006; BACASS 2008; ICSS 2010), while four trials included participants with both symptomatic and asymptomatic stenosis (TESCAS-C 2006; Beijing 2009; CREST 2010; Ostrava 2014). Where possible, we included only data from symptomatic participants enrolled in CREST 2010 and Ostrava 2014 in this comparison; in the TESCASC 2006 and Beijing 2009 trials, outcome events from symptomatic and asymptomatic participants were not available separately. These trials were included as a whole in a subgroup. The trials did not specifically include participants perceived to be at increased risk from surgery.

\section{Safety analysis: outcome events occurring between randomisation and 30 days after treatment}

Data on the primary safety outcome measure of this review, death or stroke of any severity occurring between randomisation and 30 days following the procedure, were available from participants with symptomatic carotid stenosis randomised in 10 trials. Data from two of these trials also included asymptomatic participants. These were analysed in a subgroup. There was a significant excess of the primary safety outcome measure in the stenting group (OR 1.70, $95 \% \mathrm{Cl} 1.31$ to $2.19 ; \mathrm{P}<0.0001, \mathrm{I}^{2}=5 \%$; high-certainty evidence; Analysis 1.1).

Among the six trials with available individual patient data, we compared the primary safety outcome in subgroups according to age. There was a significant interaction between treatment effect and age: among participants younger than 70 years, the OR of the primary safety outcome was $1.11\left(95 \% \mathrm{Cl} 0.74\right.$ to $\left.1.64 ; \mathrm{I}^{2}=0 \%\right)$; in participants 70 years or older, the OR was $2.23(95 \% \mathrm{Cl} 1.61$ to 3.08 ; $\mathrm{I}^{2}=0 \%$; $\mathrm{P}$ value for interaction $0.007, \mathrm{I}^{2}=26 \%$ ) (Analysis 1.2 ).

Six trials provided data for subgroup analysis according to sex. Treatment effects in men (OR $1.82,95 \% \mathrm{Cl} 1.10$ to $3.02 ;\left.\right|^{2}=54 \%$ ) and women (OR $1.52,95 \% \mathrm{Cl} 0.96$ to $2.41 ; \mathrm{I}^{2}=6 \%$ ) did not differ significantly from each other ( $P$ value for interaction 0.61 ; Analysis 1.3).

Seven trials specified the minimum amount of pretrial experience in carotid stenting. Among the three trials which required 10 or fewer procedures in the carotid artery, the summary treatment effect on the primary safety outcome (OR $2.21,95 \% \mathrm{Cl} 1.56$ to $3.13 ; I^{2}=0 \%$ ) did not differ significantly from the treatment effect observed in the four trials requiring higher levels of interventional experience (OR 1.37, 95\% Cl 0.98 to 1.94; $\mathrm{P}$ value for interaction 0.06 , $1^{2}=1 \%$; Analysis 1.4). However, in the inexperienced centres the $95 \% \mathrm{Cl}$ did not include 1, therefore participants treated by carotid stenting had a significantly worse outcome than those treated by endarterectomy. In contrast, in centres with more experience, the $95 \% \mathrm{Cl}$ included 1 , therefore in these centres there was no significant difference in primary safety outcome between the stenting and the endarterectomy groups.

There was a statistically non-significant trend towards a higher risk of the combined outcome measure of death or major or disabling stroke between carotid stenting and endarterectomy, which was reported in seven trials (OR $1.36,95 \% \mathrm{Cl} 0.97$ to $1.91 ; \mathrm{P}=0.08, \mathrm{I}^{2}=$ $0 \%$; high-certainty evidence; Analysis 1.5 ). 
Death from any cause up to 30 days after treatment was reported in 10 trials; from two of these trials, both symptomatic and asymptomatic participants were included in the comparison because mortality outcomes were not provided separately (TESCAS-C 2006; Beijing 2009). There was a non-significant increase in all-cause mortality among participants randomised to carotid stenting compared with those assigned surgery (OR 1.35, 95\% Cl 0.69 to $2.61 ; P=0.38, I^{2}=0 \%$; Analysis 1.6 ).

Among the eight trials separately reporting periprocedural stroke, carotid stenting was associated with a significant increase in the risk of stroke of any severity between randomisation and 30 days after treatment compared with endarterectomy (OR 1.78, 95\% Cl 1.38 to 2.29; $\mathrm{P}<0.00001, \mathrm{I}^{2}=0 \%$; Analysis 1.7 ). There were more fatal, disabling, or major strokes in the endovascular groups, but the difference did not reach statistical significance (OR $1.34,95 \% \mathrm{Cl}$ 0.93 to $1.92 ; \mathrm{P}=0.11, \mathrm{I}^{2}=0 \% ; 7$ trials; Analysis 1.8).

The risk of fatal or non-fatal myocardial infarction up to 30 days after treatment was lower in participants assigned to stenting than in the endarterectomy arms, among eight trials with available data (OR $0.47,95 \% \mathrm{Cl} 0.24$ to $0.94 ; \mathrm{P}=0.03, \mathrm{I}^{2}=0 \%$; Analysis 1.9 ). Combining myocardial infarction with periprocedural death or any stroke favoured endarterectomy $(\mathrm{OR} 1.43,95 \% \mathrm{Cl} 1.14$ to $1.80 ; \mathrm{P}=$ $0.002, \mathrm{I}^{2}=0 \%$; 6 trials; Analysis 1.10).

\section{Combined safety and efficacy analysis: outcome events occurring between randomisation and end of follow-up}

The primary combined safety and efficacy outcome measure, the combination of death or any stroke occurring between randomisation and 30 days after treatment or ipsilateral stroke until the end of follow-up could be extracted from eight trials, one also including participants with asymptomatic stenosis. The length of follow-up differed between trials. Six trials provided data on extensive follow-up periods of four years or longer. The primary combined outcome occurred significantly more often among participants randomised to stenting than among those allocated to endarterectomy (OR $1.51,95 \% \mathrm{Cl} 1.24$ to 1.85 ; $\mathrm{P}<$ $0.0001, I^{2}=0 \%$; Analysis 1.11). The occurrence of death or any stroke between randomisation and end of available follow-up, which was reported in nine trials, two of which also included participants with asymptomatic stenosis, was also more common in carotid stenting (OR $1.23,95 \% \mathrm{Cl} 1.03$ to $1.46 ; \mathrm{P}=0.02, \mathrm{I}^{2}=26 \%$; high-certainty evidence; Analysis 1.12).

\section{Efficacy analysis: outcome events occurring after the periprocedural period}

In the comparison of treatment efficacy regarding prevention of recurrent strokes, events occurring in the periprocedural period (i.e. between randomisation and 30 days after treatment) were excluded, and participants who died in the periprocedural period were subtracted from the total numbers of participants analysed. The EVA-3S 2006, CREST 2010, and ICSS 2010 trials separately reported numbers of strokes occurring during follow-up, which excluded events occurring within 30 days of treatment, while also providing the numbers of participants who had a stroke between randomisation and treatment. For the SPACE 2006 trial, we calculated the numbers of non-procedural strokes by subtracting the numbers of periprocedural events (which in those trials were defined as having occurred between randomisation and 30 days after treatment) from the numbers of all events occurring between randomisation and end of follow-up.
Excluding events occurring between randomisation and 30 days after treatment, during follow-up there was neither a significant difference in the occurrence of strokes in any territory (OR 1.15, $95 \% \mathrm{Cl} 0.82$ to $1.62 ; \mathrm{P}=0.4, \mathrm{I}^{2}=42 \% ; 6$ trials; Analysis 1.13$)$, nor in ipsilateral strokes $\left(\mathrm{OR} 1.05,95 \% \mathrm{Cl} 0.75\right.$ to $1.47 ; \mathrm{P}=0.77, \mathrm{I}^{2}=0 \% ; 6$ trials; Analysis 1.14).

\section{Comparison 2: stenting or endarterectomy for asymptomatic carotid stenosis at standard surgical risk}

Outcomes were available from five trials including participants with asymptomatic carotid stenosis only (Kentucky 2004; Houston 2014; ACT-1 2016; SPACE-2 2016; Carmel Medical Center 2017), and from subgroups of participants with asymptomatic stenosis enrolled in two other trials (CREST 2010; Ostrava 2014).

\section{Safety analysis: outcome events occurring between randomisation and 30 days after treatment}

Although the difference in the occurrence of the primary safety outcome between carotid stenting and endarterectomy was not statistically significant, there was a trend towards a higher risk of death or any stroke between randomisation and 30 days after treatment in the endovascular treatment groups (OR 1.72, 95\% $\mathrm{Cl} 1.00$ to $2.97 ; \mathrm{P}=0.05, \mathrm{I}^{2}=0 \%$; moderate-certainty evidence; Analysis 2.1). The combined outcome measure of death or major or disabling stroke between randomisation and 30 days after treatment was reported in two trials only. These events occurred more often in the endovascular groups, but the difference did not reach statistical significance $(\mathrm{OR} 1.54,95 \% \mathrm{Cl} 0.39$ to $6.11 ; \mathrm{P}=0.54$, $\mathrm{I}^{2}=0 \%$; moderate-certainty evidence; Analysis 2.2 .

Six trials reported the occurrence of myocardial infarction between randomisation and 30 days after treatment. There were fewer myocardial infarctions in the endovascular treatment groups compared with the endarterectomy groups, but the difference did not reach statistical significance $(\mathrm{OR} 0.53,95 \% \mathrm{Cl} 0.24$ to $1.15 ; \mathrm{P}=$ $0.11, I^{2}=0 \%$; Analysis 2.3 .

The combined outcome of death or any stroke or myocardial infarction in the periprocedural period did not differ significantly between treatments (OR 1.16, 95\% $\mathrm{Cl} 0.73$ to $1.85 ; \mathrm{P}=0.52, \mathrm{I}^{2}=0 \%$; 6 trials; moderate-certainty evidence; Analysis 2.4).

\section{Combined safety and efficacy analysis: outcome events occurring between randomisation and end of follow-up}

Six trials provided data for the primary combined safety and efficacy outcome of this review. There was no significant difference in the occurrence of death or any stroke between randomisation and 30 days after treatment or ipsilateral stroke until the end of follow-up between endovascular treatment and endarterectomy (OR $1.27,95 \% \mathrm{Cl} 0.87$ to $1.84 ; \mathrm{P}=0.22, \mathrm{I}^{2}=0 \%$; Analysis 2.5 ).

Combining all periprocedural events (death or any stroke or MI) with ipsilateral stroke until the end of follow-up, there was again no significant difference between the two treatments (OR 1.10,95\% CI 0.76 to $1.59 ; \mathrm{P}=0.62, \mathrm{I}^{2}=0 \% ; 5$ trials; moderate-certainty evidence; Analysis 2.6).

\section{Comparison 3: stenting or endarterectomy in people with elevated surgical risk}

Two trials compared endovascular treatment versus endarterectomy in participants with symptomatic or asymptomatic 
carotid stenosis who were considered to be at increased risk of complications from surgery; periprocedural outcomes are provided in Analysis 3.1, Analysis 3.2, Analysis 3.3, Analysis 3.4, Analysis 3.5, and Analysis 3.6. These outcomes were not reported for participants with symptomatic or asymptomatic stenosis separately, and the Beijing 2013 trial did not report outcomes beyond six months. The SAPPHIRE 2004 trial reported no significant difference in the occurrence of the composite outcome of death or any stroke within 30 days after treatment or ipsilateral stroke or death from neurological causes up to three years after treatment, with cumulative incidences of $9.3 \%$ in the endovascular treatment group and $10.0 \%$ in the endarterectomy group. Longterm outcomes are provided in Analysis 3.7, Analysis 3.8, and Analysis 3.9.

\section{Comparison 4: restenosis in stenting or endarterectomy for symptomatic or asymptomatic carotid stenosis}

Nine trials reported numbers of participants with severe restenosis (equivalent to $70 \%$ or greater according to the measurement of stenosis used in the NASCET trial) detected on ultrasound during follow-up. Three of these trials included both asymptomatic and symptomatic participants. The overall comparison showed no statistically significant increase in severe $(70 \%$ or greater) restenosis rates among participants randomised to stenting compared with participants assigned to surgery (OR $1.21,95 \% \mathrm{Cl}$ 0.76 to $1.93 ; P=0.42, I^{2}=61 \%$; low-certainty evidence; Analysis 4.1).

The outcome of moderate or severe $(50 \%$ or greater) restenosis during follow-up was available from four trials, and occurred significantly more often in the stenting groups than in the endarterectomy groups (OR $2.00,95 \% \mathrm{Cl} 1.12$ to $3.60 ; \mathrm{P}=0.02, \mathrm{I}^{2}=$ 44\%; Analysis 4.2).

\section{Comparison 5: access complications in stenting or endarterectomy}

Thirteen trials reported rates of cranial nerve palsy, which were significantly reduced among participants treated with stenting compared with the surgical groups $(\mathrm{OR} 0.10,95 \% \mathrm{Cl} 0.06$ to 0.16 ; $\mathrm{P}$ $<0.00001, \mathrm{I}^{2}=0 \%$; Analysis 5.1).

From six trials, we could extract the numbers of the combined outcome of death or any neurological complication (including stroke or cranial nerve palsy) between randomisation and 30 days after the procedure. There was a significant reduction of this outcome with stenting (OR $0.73,95 \% \mathrm{Cl} 0.55$ to $0.98 ; \mathrm{P}=0.03, \mathrm{I}^{2}$ $=18 \%$; Analysis 5.2). Access site haematoma (requiring surgery, blood transfusion, or prolonging hospital stay, where severity was defined) was significantly less common with endovascular treatment than with endarterectomy (OR $0.32,95 \% \mathrm{Cl} 0.15$ to 0.68 ; $\mathrm{P}=0.003, \mathrm{I}^{2}=27 \%$; 10 trials; Analysis 5.3).

Treatment effects in these comparisons did not appear to differ between participants with symptomatic or asymptomatic stenosis or those at increased surgical risk.

\section{Comparison 6: endovascular treatment or medical care in participants not suitable for surgery}

Data were only available from two very small studies. There was no significant difference in the occurrence of stroke or death between randomisation and the end of follow-up (OR $0.22,95 \% \mathrm{Cl} 0.01$ to 7.92; $\mathrm{P}=0.41, \mathrm{I}^{2}=79 \%$; Analysis 6.1).
Comparison 7: balloon angioplasty with or without stent insertion or endarterectomy for symptomatic carotid stenosis

Data were only available from two studies which had already been identified during the previous update of this review (Bonati 2012). The primary safety outcome measure, death or stroke of any severity occurring between randomisation and 30 days following the procedure favoured endarterectomy, albeit with significant heterogeneity between the two trials (OR 1.76, 95\% CI 1.06 to $\left.2.92 ; P=0.03, I^{2}=77 \%\right)$. Combining the primary safety outcome with ipsilateral stroke until the end of follow-up, data were only available from the CAVATAS-CEA 2001 trial and showed no significant difference between the two treatments (OR 1.47, 95\% $\mathrm{Cl} 0.93$ to $2.33 ; \mathrm{P}=0.91)$. With regard to severe $(70 \%$ or greater) restenosis during follow-up, there was a highly significant increase in the occurrence of restenosis in the endovascular arm of the trial, in which most participants were treated by balloon angioplasty without the insertion of stents (OR 3.48, 95\% Cl 1.99 to 6.07 ; $\mathrm{P}<$ 0.0001).

\section{Comparison 8: balloon angioplasty with or without stent insertion or endarterectomy for asymptomatic carotid stenosis}

Data were only available from one study and results are provided in Analysis 8.1 and Analysis 8.2.

\section{DISCUSSION}

The purpose of this review was to summarise the evidence from randomised trials on the safety and efficacy of endovascular treatment of symptomatic or asymptomatic internal carotid artery stenosis. The previous version of this review demonstrated that among people with symptomatic carotid stenosis, carotid stenting was associated with a higher risk of periprocedural stroke or death than endarterectomy. However, at that time, little evidence was available on stenting of asymptomatic carotid stenosis and on the long-term efficacy of carotid stenting in general. The present update of this review includes new evidence from randomised trials addressing these questions as well as long-term follow-up of previously identified studies.

\section{Summary of main results}

In people with symptomatic carotid stenosis, endarterectomy was associated with a lower risk of death or any stroke (the primary safety outcome), death or any stroke or MI, as well as any stroke, occurring between randomisation and 30 days after treatment (defined as the periprocedural period). Age significantly modified the treatment effect on the primary safety outcome: rates of periprocedural death or stroke did not differ significantly in people younger than 70 years, but were significantly increased with carotid stenting compared with surgery in the older age (70 years or older) group. There was some evidence for an increased risk of major periprocedural outcome events with carotid stenting (shown for fatal, major, or disabling stroke or the combination thereof with all-cause death), but no difference in all-cause mortality. Carotid stenting was associated with lower risks of MI, cranial nerve palsy, and access site haematoma.

Including events occurring during long-term follow-up, endarterectomy was superior to carotid stenting in the comparison of death or any stroke in the periprocedural period combined with ipsilateral stroke occurring beyond (the primary combined 
safety and efficacy outcome), as well as in the comparison of death or any stroke between randomisation and the end of follow-up. Excluding periprocedural events, the rates of stroke in any territory and ipsilateral stroke did not differ between treatments.

In people with asymptomatic carotid stenosis, there was no significant difference between carotid stenting and endarterectomy in the occurrence of death or any stroke between randomisation and 30 days after treatment, but there was a strong trend towards endarterectomy showing a lower risk of death or any stroke during the periprocedural period. The combination of this outcome with ipsilateral stroke occurring during follow-up also showed a non-significant difference in favour of surgery.

Among people with symptomatic and asymptomatic carotid stenosis combined, moderate or severe restenosis during follow-up was more common after stenting than after endarterectomy, but the rate of severe carotid restenosis did not differ significantly.

\section{Overall completeness and applicability of evidence}

\section{Is stenting as safe and effective as endarterectomy in people with symptomatic carotid stenosis?}

\section{Safety}

With regard to the first main research question, there is strong evidence that in people with symptomatic carotid stenosis who can undergo surgery safely, stenting is associated with a higher risk of the combined outcome of death or stroke of any severity in the periprocedural period than endarterectomy.

There is also strong evidence that this excess risk associated with stenting is dependent on age. There was a significant interaction between participants' age and the summary treatment effect on the primary short-term outcome observed among those trials with available access to individual participant data: treatments yielded broadly similar results in participants younger than 70 years while for older participants, the odds of death or stroke up to 30 days after stenting were more than double of those receiving endarterectomy (Sedgwick 2014). Age was previously recognised as an important risk factor for stroke associated with carotid stenting (Touzé 2009), but had little effect on stroke risk in endarterectomy (Rothwell 2004; Bond 2005). A previous pooled analysis of three large European stenting trials (EVA-3S 2006; SPACE 2006; ICSS 2010), suggested that the excess in stroke risk of stenting over surgery increased steadily with patients' ages (CSTC 2010), a finding that was supported by a similar analysis in the CREST 2010 trial. An update of the aforementioned pooled analysis with individual participant data from the CREST trial confirmed these findings, while showing no significant association of participant age with the long-term risk of ipsilateral stroke after either treatment (Howard 2016). Factors mediating this association might be an increased burden of atherosclerosis in the aortic arch, plaque instability, or vessel tortuosity in elderly people, which might increase the risk of procedure-related stroke in stenting.

The current evidence does not show a relevant influence of sex on the comparative risks of stenting versus endarterectomy. Other individual factors which may determine periprocedural stroke risk, such as vascular anatomy or characteristics of the atherosclerotic plaque, could not be addressed in the present review.
The difference in the combined primary safety outcome was mainly driven by a significant increase in risk of stroke of any severity with stenting occurring up to 30 days after the procedure. However, there was also some evidence of an increased risk of fatal stroke, stroke leading to disability, or stroke which was otherwise classified as major with stenting. This difference did not reach statistical significance $(P=0.08)$, but due to the small numbers of events leading to large $\mathrm{Cls}$, the current data could not rule out an increase in strokes leading to death or disability in stenting compared with endarterectomy. Total procedural mortality (including death of any cause) did not differ significantly. MI occurred less often with stenting than with endarterectomy, but the comparison of periprocedural death, stroke, or MI combined still favoured endarterectomy.

\section{Efficacy}

Since the last update, four large randomised trials have published event rates beyond four years of follow-up (Kentucky 2001; EVA-3S 2006; CREST 2010; ICSS 2010). The primary combined safety and efficacy outcome, the combination of death or any stroke between randomisation and 30 days after treatment or ipsilateral stroke during follow-up, as well as of death or any stroke between randomisation and end of follow-up favoured endarterectomy. The same was true when periprocedural MI was added. However, there was no significant difference in the rate of stroke in any territory, or ipsilateral stroke, if events occurring up to 30 days after treatment were excluded. These findings demonstrate that the lower net benefit of stenting in preventing strokes is attributable to a higher periprocedural stroke risk. Beyond the periprocedural period, there is now strong evidence that stenting and endarterectomy are equally effective in preventing recurrent stroke in the long-term.

\section{Is stenting as safe and effective as endarterectomy in people with asymptomatic stenosis?}

Evidence on people with asymptomatic carotid stenosis is now available from more than 3000 participants treated in randomised trials. There is some evidence for an increased risk of periprocedural death or stroke with stenting compared with endarterectomy, although this difference did not reach statistical significance. Compared with endarterectomy, the combined periprocedural outcome of death or fatal, disabling, or major stroke occurred more often after stenting and periprocedural MI less often. These effects were not statistically significant but the relatively small numbers of events do not allow firm conclusions. The combined periprocedural outcome of death, stroke, or MI did not differ between treatments.

The combined safety and efficacy comparison including death or any stroke in the periprocedural period or ipsilateral stroke thereafter also showed no significant difference between the two treatments.

Cls surrounding the effect measures in the comparisons of participants with asymptomatic carotid stenosis were much wider than in the comparisons of participants with symptomatic stenosis. Also, it has to be noted that only one large trial reported long-term outcomes (CREST 2010).

Therefore, the existing evidence does not yet allow any firm conclusions on the comparative safety and efficacy of stenting versus endarterectomy in people with asymptomatic carotid stenosis. The data urge caution and the results of the ongoing 
large ACST-2 trial, as well as extended follow-up data from ACT-1 2016 and SPACE-2 2016 are needed. The question of whether any form of revascularisation is beneficial for asymptomatic people in comparison to optimised medical treatment using current regimens is also an important issue that is being addressed by ongoing trials (CREST-2; ECST-2; NCT02841098).

\section{Is stenting safe and effective in people considered at increased surgical risk?}

Limited data are available on stenting or endarterectomy in people who were perceived to be at increased risk with surgery, based on cardiopulmonary comorbidity, stenosis following previous endarterectomy or irradiation, or contralateral carotid occlusion. Two trials originally reported non-inferiority of stenting in terms of the primary endpoint death, stroke, or MI occurring up to 30 days after treatment, or death or ipsilateral stroke in the first year after treatment (SAPPHIRE 2004; Beijing 2013). It has to be noted that in the SAPPHIRE 2004 trial, the definition of MI used in the primary analysis was based solely on the basis of elevated cardiac enzymes. Moreover, neither of the two trials reported the combined outcome of death or any stroke in the periprocedural period, and results were not provided according to symptom status. Less than $30 \%$ of study participants included in the SAPPHIRE 2004 trial had symptomatic stenosis and both trials included a heterogeneous group of participants with various conditions thought to increase the risk of endarterectomy, which makes it difficult to form conclusions about the appropriateness of revascularisation using stenting (or endarterectomy) within the various groups included in these trials.

\section{Does the risk of restenosis of the carotid artery differ between stenting and endarterectomy?}

Ten trials reported long-term restenosis rates. The pooled results of the more recent trials comparing primary stenting versus endarterectomy showed no significant increase in severe restenosis, albeit with evidence of substantial heterogeneity $\left(I^{2}=\right.$ $58 \%$ ). If only trials in which mostly balloon angioplasty without stenting were considered, there was a highly significant increase in severe restenosis after balloon angioplasty compared with endarterectomy (CAVATAS-CEA 2001).

Moderate or severe restenosis $(50 \%$ or greater) occurred significantly more often after stenting than after endarterectomy.

Some trials reported data on whether restenosis was associated with recurrent stroke. The authors of the ICSS 2010 trial found an increase in the occurrence of ipsilateral stroke after moderate or severe $(50 \%$ or greater) restenosis in both treatment groups combined. Participants with $50 \%$ or greater restenosis had a higher risk of ipsilateral stroke than people without restenosis in the carotid endarterectomy group alone but not in the carotid angioplasty and stenting group. In the EVA-3S 2006 trial, the investigators found no effect of restenosis on the risk of ipsilateral stroke. CREST 2010 reported a higher risk of ipsilateral stroke after the periprocedural period up to the end of follow-up in people who had restenosis of $70 \%$ or greater. The SPACE 2006 trial reported no differences of ipsilateral stroke in people with restenosis compared with people without restenosis in either treatment group.

No information on clinical consequences of restenosis (severe or moderate) was available from Kentucky 2001 and Kentucky 2004 trials.
One systematic review investigating whether severe restenosis (greater than $70 \%$ ) after carotid endarterectomy or endovascular treatment increases the risk of ipsilateral stroke included data from 11 randomised trials (Kumar 2017), six of which are included in this review (CAVATAS-CEA 2001; EVA-3S 2006; SPACE 2006; Regensburg 2008; CREST 2010; ICSS 2010). In this meta-analysis, participants with severe restenosis after endarterectomy were at significantly higher risk of late ipsilateral stroke than participants without restenosis. There was no significant difference in the rate of ipsilateral stroke in participants with severe restenosis after endovascular therapy compared to participants without severe restenosis (Kumar 2017).

\section{Do the rates of cranial nerve palsy and other vascular complications differ between stenting and endarterectomy?}

There is strong evidence that stenting less often causes cranial nerve palsy and access site haematoma, and some evidence that it is associated with a lower risk of MI compared with endarterectomy. However, trials differed in their assessment and definitions of MI. Among trials which reported MI as an endpoint, two used the WHO definition (EVA-3S 2006; ICSS 2010), which includes two out of the following three criteria: prolonged typical chest pain, elevation of specific cardiac enzymes more than twice the upper limit of normal, and specific ECG abnormalities. Three trials routinely screened their participants with ECG and cardiac enzyme measurement before and after treatment, albeit definitions of MI still differed (CREST 2010; Ostrava 2014; ACT-1 2016). SPACE 2006 did not include $\mathrm{MI}$ as a predefined endpoint, but for the purpose of a previous pooled analysis, the investigators reassessed all adverse event reports and did not find any reported MIs in the periprocedural period fulfilling WHO criteria (CSTC 2010). Two trials reported numbers of MIs without providing definitions (CAVATASCEA 2001; BACASS 2008). The Kentucky 2001 trial reported that one participant died of an MI immediately after endarterectomy but did not specify inclusion of non-fatal MI as an outcome measure; therefore, we excluded this trial from the comparison of all MIs in this review. There was no information on MI available from the remaining trials.

Despite of these differences, relative effect measures in our analyses showed no substantial heterogeneity.

\section{Is endovascular treatment safe and effective in people unsuitable for surgery?}

We only identified two very small trials comparing carotid stenting with best medical care in people not well enough to undergo surgery. There was substantial heterogeneity in treatment effects between them $\left(I^{2}=79 \%\right)$. Although presented here, these results do not justify any conclusion.

\section{Are the results of this review applicable to clinical practice?}

Centres that took part in the trials included in this review had a specific interest in secondary prevention of stroke and care must be taken when extrapolating the data into routine clinical practice in less-specialised centres. However, contrary concerns - that the unfavourable stroke rates observed in some of the trials was the consequence of including centres with insufficient experience with the procedure - have also been voiced (Forsting 2007; Roffi 2010). Therefore, we included a comparison of the primary safety outcome according to the number of stenting procedures in the carotid artery interventionalists needed to have performed 
before joining the trials. Indeed, point estimates of ORs favoured surgery more strongly among trials that required 10 or fewer pretrial procedures than among those requiring more. Moreover, the interaction between the number of pretrial procedures and treatment effect was borderline significant. Importantly, point estimates of effect measures still favoured endarterectomy among the trials with higher experience.

Previous data on the role of experience from some of these trials has been somewhat conflicting. In the SPACE 2006 study, centrespecific periprocedural stroke or death rates in the stenting arm decreased with increasing total number of participants enrolled at the centres. The investigators of the EVA-3S 2006 trial analysed outcomes after stenting on the level of individual interventionalists according to the number of procedures they had performed before the trial. Somewhat unexpectedly, the complication rate was lowest in the least experienced group. A previously published pooled analysis of individual participant data from EVA-3S 2006, SPACE 2006, and ICSS 2010 showed that the excess stroke or death risk associated with stenting was higher among centres enrolling fewer than 50 participants into the trial than in the larger centres, but again the Cls of the effect measures in higher- and lowerrecruiting centres overlapped widely and the risk of stenting was still higher than endarterectomy in the larger centres (CSTC 2010). Thus, while it stands to reason that the risks of stenting will decline with experience, the current evidence does not suggest that the higher stroke rates associated with stenting observed in clinical trials is mainly explained by lack of experience at the centres included in the trials. Therefore, we consider that, for those research questions for which there was sufficient evidence, the findings of this review are applicable to routine clinical practice.

Some of the trials included in this review enrolled participants in the early 2000s. The periprocedural risk in both treatment groups may have decreased since then. Particularly in stenting, many technical developments, such as mesh-covered stent devices or stents with very small open area between struts, reverseflow protection systems, and direct transcervical access were only introduced recently and were not widely available at the time most of the trials included in this review were conducted. Thus, some of the findings of this meta-analysis may no longer be applicable to current clinical practice.

The results of the trials enrolling participants at increased surgical risk are difficult to extrapolate into clinical practice, mostly because outcomes were not provided according to symptom status and due to the heterogeneous indications for inclusion in the trial. Many physicians may opt in favour of conservative treatment in these patients if they have asymptomatic carotid stenosis. For this reason, we decided against pooling data from these trials with data from the remaining trials that included participants at standard surgical risk.

\section{Quality of the evidence}

With regard to the comparison of the primary safety outcome, there was only negligible heterogeneity between treatment effects observed in contributing trials $\left(I^{2}=5 \%\right.$ ) (Analysis 1.1). There was even less evidence of heterogeneity between trials within the two analysed age subgroups (younger than 70 years: $\mathrm{I}^{2}=0 \% ; 70$ years or older: $I^{2}=0 \%$ ) (Analysis 1.2). Some of the heterogeneity observed in the main comparison might therefore be accounted for by differences in population age.
However, there are several other possible reasons for the heterogeneity of data between trials that were not formally examined. The stenting technique used was not the same for all the trials. We included in the meta-analysis results acquired before standardisation of the stenting technique (Kentucky 2001; WALLSTENT 2001). In addition, some of the devices used for stenting in the trials conducted in the early 2000 s are now also outdated and no longer in widespread use (EVA-3S 2006; SPACE 2006; CREST 2010; ICSS 2010).

Heterogeneity may also have been caused by differences in baseline characteristics of the participants in the trials other than age. We addressed differences in symptom status by performing separate comparisons for participants with symptomatic or asymptomatic stenosis where this was possible. Data from SAPPHIRE 2004 and Beijing 2013, which specifically selected participants if they were at high surgical risk, were separated from the other trials. However, although the CAVATAS protocol did not specify high risk as an inclusion criterion, comparison of baseline characteristics suggested that CAVATAS-CEA 2001 also selected a higher proportion of participants at high surgical risk compared with ECST 1998 and NASCET 1991. Furthermore, history of cardiovascular disease was more common in the CREST 2010 trial (also among the subgroup of symptomatic participants), than in the large European trials (EVA-3S 2006; SPACE 2006; ICSS 2010).

Heterogeneity may have arisen because we included both completed trials and trials that stopped recruitment before reaching the planned sample size: three trials were terminated early because of concerns over the safety of stenting (Leicester 1998; WALLSTENT 2001; EVA-3S 2006), while five trials were stopped for other reasons (SAPPHIRE 2004; SPACE 2006; BACASS 2008; Regensburg 2008; ACT-1 2016).

The Leicester 1998 trial, which planned to enrol 300 participants, was suspended after referral to the Data Monitoring Committee who invoked the stopping rule. A total of 23 participants were randomised, 17 of whom received their allocated treatment. Five of the seven participants undergoing endovascular treatment had strokes (three of which were disabling at 30 days) compared with 10 uncomplicated carotid endarterectomies $(P=0.0034)$. The investigators subsequently felt that the trial could not be restarted even in an amended form primarily because of difficulties with informed consent. The WALLSTENT 2001 trial was stopped after 219 participants had been enrolled (planned sample size 700 participants). The primary endpoint was the cumulative occurrence of ipsilateral stroke, procedure-related death, or vascular death within one year. It was reported that the primary endpoint rate after approximately one year was $12.1 \%$ in the stenting group and $3.6 \%$ in the endarterectomy group $(P=0.022)$ and that the $30-$ day periprocedural stroke or death rate was $12.1 \%$ in the stenting group and $4.4 \%$ in the endarterectomy group $(P=0.049)$. The decision to terminate the study was based on the data and a futility analysis. However, it has been reported that at a meeting of the American Stroke Association, the trial was criticised first because it was stopped by the sponsor, rather than by the Data Safety and Monitoring Board, even though the outcome in all participants had not been validated, and second because the competence and experience of those undertaking stenting was questioned (American Stroke Association). As we have not yet obtained detailed data for this study, we can make no further assessment of its quality at this time. The EVA-3S 2006 trial was stopped by 
the Safety Committee after 527 participants had been randomised due to safety and futility concerns. The 30-day periprocedural stroke or death rate was $9.6 \%$ in the stenting arm and $3.9 \%$ in the endarterectomy arm $(P=0.01)$. This non-inferiority trial originally planned to enrol 827 participants. However, based on the observed risks, 4000 participants would have been necessary to demonstrate non-inferiority of stenting with the predefined margin of a $2 \%$ absolute difference in 30-day stroke or death rates. The SAPPHIRE 2004 trial did not specify a target sample size. Instead, it was planned to terminate the trial as soon as a non-inferiority margin of $3 \%$ absolute difference in either the 30-day death, stroke, or MI rate (in all participants), or in the primary endpoint, which also included ipsilateral stroke or death from neurological causes up to one year after treatment (in those participants followed up for one year) could be demonstrated. However, randomisation was stopped after 334 participants in early 2002, citing an abrupt fall in the enrolment rate as the number of participants receiving stent treatment in non-randomised registries increased. The SPACE 2006 trial originally planned to include 1900 participants; the Steering Committee stopped the trial after two interim analyses (one at 950 participants and one at 1200 participants), which revealed that more than 2500 participants would have been necessary to demonstrate non-inferiority of stenting with the predefined margin of $2.5 \%$ absolute difference in the primary outcome measure (death or ipsilateral ischaemic stroke between randomisation and 30 days after treatment), also considering the lack of further funding. The ACT-1 2016 trial originally planned to enrol 1658 participants, but the study was terminated early due to slow enrolment and enrolled only 1453 participants. Two small singlecentre studies were terminated when the large multicentre trials started recruitment: BACASS 2008 was stopped after randomising 20 participants, when the centre joined ICSS 2010; the Regensburg 2008 study ended randomisation after 87 of the 200 originally planned participants upon initiation of the SPACE 2006 trial.

There was substantial heterogeneity in the assessment of moderate or severe restenosis between trials (Analysis 4.1; Analysis 4.2). Some heterogeneity may have been caused by differences in participant characteristics as we included both participants with asymptomatic and symptomatic carotid stenosis in these analyses.

There was only negligible heterogeneity between treatment effects observed in the contributing trials with regard to our primary safety outcome in participants with symptomatic carotid stenosis $\left(1^{2}=5 \%\right)$ and no heterogeneity with regard to the primary safety outcome in participants with asymptomatic carotid stenosis. However, there was substantial heterogeneity in some of the secondary analyses. With regards to risk of bias, some of the small randomised studies we included were subject to unclear risks of bias. In addition, we downgraded the level of evidence for the comparisons in participants with asymptomatic carotid stenosis due to imprecision of the effect estimates. Overall, we feel that the quality of the evidence was sufficient to address the principal research questions of this review.

\section{AUTHORS' CONCLUSIONS}

\section{Implications for practice}

In people with symptomatic carotid stenosis, stenting is associated with a higher risk of periprocedural stroke or death compared with endarterectomy. However, both procedures are equally effective at preventing stroke in the long term. Therefore, the choice between the two procedures should be based on minimising periprocedural risks. For this reason, symptomatic carotid stenosis should not be routinely treated with carotid stenting in people above the age of 70 years, provided the people are fit and willing to undergo surgery, and endarterectomy can be performed at standard risk. Stenting can be offered as an alternative to endarterectomy to people with symptomatic stenosis who are younger than 70 years at centres achieving periprocedural stroke or death rates in this age group comparable to those with endarterectomy. Factors such as patients' preference, cardiovascular risk, and vascular anatomy should also be taken into consideration in the choice between the two procedures.

There is still insufficient evidence on short-term and longterm outcomes to justify stenting as a routine alternative to endarterectomy for asymptomatic carotid stenosis.

Stenting may be considered in people with symptomatic carotid stenosis deemed to require revascularisation in whom surgery is contraindicated, associated with a high risk of complications, or technically not feasible, so long as the risk of recurrent symptoms with optimised medical treatment alone is considered greater than the risk of the stenting procedure. However, randomised trial data are sparse in these people, and the optimal management is often unclear.

\section{Implications for research}

The existing data support the continuing inclusion of people with asymptomatic carotid stenosis thought to require revascularisation in randomised clinical trials comparing stenting versus endarterectomy. At present only ACST-2 is still recruiting participants deemed to require revascularisation. If the clinician is uncertain whether revascularisation provides benefit over modern medical treatment alone, patients should be randomised in CREST-2 (including people with asymptomatic carotid stenosis) or in ECST-2 (including people with asymptomatic or low- to intermediate-risk symptomatic stenosis).

\section{ACKNOWLEDGEMENTS}

We would like to thank Mrs Hazel Fraser and Mr Josh Cheyne at the Cochrane Stroke Group for searching the trials register and for help in defining the search strategy. We would like to acknowledge $\mathrm{Dr}$ Francesca Crawley and Dr Lucy Coward, the first authors of the first two versions of this review. We would also like to acknowledge $\mathrm{Dr}$ Jörg Ederle for his contributions to the previous review.

Professor Leo Bonati was supported in part by grants from the Swiss National Science Foundation (PBBSB-116873), the University of Basel, Switzerland, and The Stroke Association. He received grants for research projects on carotid stenosis from the Swiss National Science Foundation (32003B-156658), the University of Basel, the Swiss Heart Foundation, and AstraZeneca. Professor Martin Brown's Chair in Stroke Medicine was supported by The Reta Lila Weston Trust for Medical Research.

If anyone is aware of completed or ongoing trials of carotid angioplasty and stenting not included in this review, please contact Professor Bonati. 


\section{REFERE N CE S}

\section{References to studies included in this review}

ACT-1 2016 \{published data only\}

Rosenfield K, Matsumura JS, Chaturvedi S, Riles T, Ansel GM, Metzger DC, ACT-I Investigators. Randomized trial of stent versus surgery for asymptomatic carotid stenosis. New England Journal of Medicine 2016;374(11):1011-20.

\section{BACASS 2008 \{published data only\}}

Hoffmann A, Engelter S, Taschner C, Mendelowitsch A, Merlo A, Radue EW, et al. Carotid artery stenting versus carotid endarterectomy - a prospective randomised controlled singlecentre trial with long-term follow up (BACASS). Schweizer Archiv für Neurologie und Psychiatrie 2008;159:84-9.

\section{Beijing 2003 \{published data only\}}

Zhao XL, Jia JP, Ji XM, Peng M, Ling F. A follow-up: stroke in patients with bilateral severe carotid stenosis after intervention treatment. Chinese Journal of Clinical Rehabilitation 2003;7(19):2714-5.

\section{Beijing 2009 \{published data only\}}

Liu CW, Liu B, Ye W, Wu WW, Li YJ, Zheng YH, et al. Carotid endarterectomy versus carotid stenting: a prospective randomized trial. Zhonghua Wai Ke za Zhi [Chinese Journal of Surgery] 2009;47(4):267-70.

\section{Beijing 2013 \{published data only\}}

Wang P, Liang C, Du J, Li J. Effects of carotid endarterectomy and carotid artery stenting on high-risk carotid stenosis patients. Pakistan Journal of Medical Sciences 2013;29(6):1315-8.

\section{Carmel Medical Center 2017 \{published data only\}}

Mannheim D, Karmeli R. A prospective randomized trial comparing endarterectomy to stenting in severe asymptomatic carotid stenosis. Journal of Cardiovascular Surgery 2017;58(6):814-7.

\section{CAVATAS-CEA 2001 \{published data only\}}

Bonati LH, Ederle J, McCabe DJ, Dobson J, Featherstone RL, Gaines PA, CAVATAS Investigators. Long-term risk of carotid restenosis in patients randomly assigned to endovascular treatment or endarterectomy in the Carotid and Vertebral Artery Transluminal Angioplasty Study (CAVATAS): long-term follow-up of a randomised trial. Lancet Neurology 2009;8(10):908-17.

Brown MM. Carotid And Vertebral Artery Transluminal Angioplasty Study. Stroke 1994;25:542.

CAVATAS Investigators. CAVATAS - Carotid and Vertebral Artery Transluminal Angioplasty Study. Unpublished protocol February 2006.

* CAVATAS Investigators. Endovascular versus surgical treatment in patients with carotid stenosis in the Carotid And Vertebral Artery Transluminal Angioplasty Study (CAVATAS): a randomised trial. Lancet 2001;357(9270):1729-37.
Ederle J, Bonati LH, Dobson J, Featherstone RL, Gaines PA, Beard JD, CAVATAS Investigators. Endovascular treatment with angioplasty or stenting versus endarterectomy in patients with carotid artery stenosis in the Carotid and Vertebral Artery Transluminal Angioplasty Study (CAVATAS): long-term follow-up of a randomised trial. Lancet Neurology 2009;8(10):898-907.

\section{CAVATAS-MED 2009 \{published data only\}}

Ederle J, Featherstone RL, Brown MM, CAVATAS Collaborators. Long-term outcome of endovascular treatment versus medical care for carotid artery stenosis in patients not suitable for surgery and randomised in the Carotid And Vertebral Artery Transluminal Angioplasty study (CAVATAS). Cerebrovascular Diseases 2009;28(1):1-7.

\section{CREST 2010 \{published and unpublished data\}}

* Brott TG, Hobson RW, Howard G, Roubin GS, Clark WM, Brooks W, CREST Investigators. Stenting versus endarterectomy for treatment of carotid-artery stenosis. New England Journal of Medicine 2010;363(1):11-23.

Brott TG, Howard G, Roubin GS, Meschia JF, Mackey A, Brooks W, CREST Investigators. Long-term results of stenting versus endarterectomy for carotid-artery stenosis. New England Journal of Medicine 2016;374(11):1021-31.

Hobson RW. Update on the Carotid Revascularization Endarterectomy versus Stent Trial (CREST) protocol. Journal of the American College of Surgeons 2002;194(S1):9-14.

Hobson RW 2nd. CREST (Carotid Revascularization Endarterectomy versus Stent Trial): background, design, and current status. Seminars in Vascular Surgery 2000;13(2):139-43.

Howard VJ, Lutsep HL, Mackey A, Demaerschalk BM, Sam AD 2nd, Gonzales NR, CREST investigators. Influence of sex on outcomes of stenting versus endarterectomy: a subgroup analysis of the Carotid Revascularization Endarterectomy versus Stenting Trial (CREST). Lancet Neurology 2011;10(6):530-7.

Lal BK, Beach KW, Roubin GS, Lutsep HL, Moore WS, Malas MB, CREST Investigators. Restenosis after carotid artery stenting and endarterectomy: a secondary analysis of CREST, a randomised controlled trial. Lancet Neurology 2012;11(9):755-63.

Sheffet AJ, Roubin G, Howard G, Howard V, Moore W, Meschia JF, et al. Design of the Carotid Revascularization Endarterectomy vs. Stenting Trial (CREST). International Journal of Stroke 2010;5(1):40-6

Silver FL, Mackey A, Clark WM, Brooks W, Timaran CH, Chiu D, CREST Investigators. Safety of stenting and endarterectomy by symptomatic status in the Carotid Revascularization Endarterectomy Versus Stenting Trial (CREST). Stroke 2011;42(3):675-80.

EVA-3S 2006 \{published and unpublished data\}

Arquizan C, Trinquart L, Touboul PJ, Long A, Feasson S, Terriat B, EVA-3S Investigators. Restenosis is more frequent after carotid 
stenting than after endarterectomy: the EVA-3S study. Stroke 2011;42(4):1015-20.

EVA-3S Investigators. Carotid angioplasty and stenting with and without cerebral protection: clinical alert from the Endarterectomy Versus Angioplasty in patients with Symptomatic Severe carotid Stenosis (EVA-3S) trial. Stroke 2004;35(1):e18-20.

EVA-3S Investigators. Endarterectomy Versus Angioplasty in patients with Symptomatic Severe carotid Stenosis (EVA-3S) trial. Cerebrovascular Diseases 2004;18(1):62-5.

Mas JL, Arquizan C, Calvet D, Viguier A, Albucher JF, Piquet P, EVA-3S Investigators. Long-term follow-up study of endarterectomy versus angioplasty in patients with symptomatic severe carotid stenosis trial. Stroke 2014;45(9):2750-6.

* Mas JL, Chatellier G, Beyssen B, Branchereau A, Moulin T, Becquemin JP, EVA-3S Investigators. Endarterectomy versus stenting in patients with symptomatic severe carotid stenosis. New England Journal of Medicine 2006;355(16):1660-71.

Mas JL, Chatellier G, EVA-3S Investigators. Endarterectomy versus stenting for carotid stenosis - authors' reply. New England Journal of Medicine 2007;356(3):306-7.

Mas JL, Trinquart L, Leys D, Albucher JF, Rousseau H, Viguier A, EVA-3S Investigators. Endarterectomy Versus Angioplasty in Patients with Symptomatic Severe Carotid Stenosis (EVA-3S) trial: results up to 4 years from a randomised, multicentre trial. Lancet Neurology 2008;7(10):885-92.

\section{Houston 2014 \{published data only\}}

Kougias P, Collins R, Pastorek N, Sharath S, Barshes NR, McCulloch K, et al. Comparison of domain-specific cognitive function after carotid endarterectomy and stenting. Journal of Vascular Surgery 2015;62(2):355-61.

\section{ICSS 2010 \{published and unpublished data\}}

Altinbas A, van Zandvoort MJ, van den Berg E, Jongen LM, Algra A, Moll FL, et al. Cognition after carotid endarterectomy or stenting: a randomised comparison. Neurology 2011;77(11):1084-90.

Bonati LH, Dobson J, Featherstone RL, Ederle J, van der Worp HB, de Borst GJ, ICSS Investigators. Longterm outcomes after stenting versus endarterectomy for treatment of symptomatic carotid stenosis: the International Carotid Stenting Study (ICSS) randomised trial. Lancet 2014;385(9967):529-38.

Bonati LH, Gregson J, Dobson J, McCabe DJ, Nederkoorn PJ, van der Worp HB, ICSS Investigators. Restenosis and risk of stroke after stenting or endarterectomy for symptomatic carotid stenosis in the International Carotid Stenting Study (ICSS): secondary analysis of a randomised trial. Lancet Neurology 2018;17(7):587-96.

Bonati LH, Jongen LM, Haller S, Flach HZ, Dobson J, Nederkoorn PJ, ICSS MRI study group. New ischaemic brain lesions on MRI after stenting or endarterectomy for symptomatic carotid stenosis: a substudy of the International Carotid Stenting Study (ICSS). Lancet Neurology 2010;9(4):353-62.

Featherstone RL, Brown MM, Coward LJ, ICSS Investigators. International carotid stenting study: protocol for a randomised clinical trial comparing carotid stenting with endarterectomy in symptomatic carotid artery stenosis. Cerebrovascular Diseases 2004;18(1):69-74.

ICSS - International Carotid Stenting Study. Protocol Version 3.2. www.ion.ucl.ac.uk/cavatas_icss/protocol.pdf (last accessed 15 April 2011).

* International Carotid Stenting Study investigators. Carotid artery stenting compared with endarterectomy in patients with symptomatic carotid stenosis (International Carotid Stenting Study): an interim analysis of a randomised controlled trial. Lancet 2010;375(9719):985-97.

\section{Kentucky 2001 \{published data only\}}

Brooks WH, Jones MR, Gisler P, McClure RR, Coleman TC, Breathitt $L$, et al. Carotid angioplasty with stenting versus endarterectomy: 10-year randomized trial in a community hospital. Journal of the American College of Cardiology: Cardiovascular Interventions 2013;7(2):163-8.

* Brooks WH, McClure RR, Jones MR, Coleman TC, Breathitt L. Carotid angioplasty and stenting versus carotid endarterectomy: randomized trial in a community hospital. Journal of the American College of Cardiology: Cardiovascular Interventions 2001;38(6):1589-95.

\section{Kentucky 2004 \{published data only\}}

Brooks WH, Jones MR, Gisler P, McClure RR, Coleman TC, Breathitt $L$, et al. Carotid angioplasty with stenting versus endarterectomy: 10-year randomized trial in a community hospital. Journal of the American College of Cardiology: Cardiovascular Interventions 2013;7(2):163-8.

* Brooks WH, McClure RR, Jones MR, Coleman TL, Breathitt L. Carotid angioplasty and stenting versus carotid endarterectomy for treatment of asymptomatic carotid stenosis: a randomized trial in a community hospital. Neurosurgery 2004;54(2):318-24.

\section{Leicester 1998 \{published data only\}}

Naylor A, Bell P, Bolia A, Abbott R, Pye I, Robertson I, et al. A randomised controlled study to compare carotid endarterectomy with protected carotid angioplasty. Unpublished protocol 1996.

* Naylor AR, Bolia A, Abbott RJ, Pye IF, Smith J, Lennard N, et al. Randomized study of carotid angioplasty and stenting versus carotid endarterectomy: a stopped trial. Journal of Vascular Surgery 1998;28(2):326-34.

\section{Ostrava 2014 \{published and unpublished data\}}

Kuliha M, Roubec M, Prochazka V, Jonszta T, Hrbac T, Havelka J, et al. Randomized clinical trial comparing neurological outcomes after carotid endarterectomy or stenting. British Journal of Surgery 2014;102:194-201. 


\section{Regensburg 2008 \{published data only\}}

Link J, Manke C, Rosin L, Borisch I, Topel I, Horn M, et al. Carotid endarterectomy and carotid stenting. Pilot study of a prospective, randomized and controlled comparison [Karotisendarterektomie und Karotisstenting. Pilotstudie eines prospektiven, randomisierten und kontrollierten Vergleichs]. Radiologe 2000;40(9):813-20.

* Steinbauer MG, Pfister K, Greindl M, Schlachetzki F, Borisch I, Schuirer G, et al. Alert for increased long-term follow-up after carotid artery stenting: results of a prospective, randomized, single-center trial of carotid artery stenting vs carotid endarterectomy. Journal of Vascular Surgery 2008;48(1):93-8.

\section{SAPPHIRE 2004 \{published data only\}}

Gurm HS, Yadav JS, Fayad P, Katzen BT, Mishkel GJ, Bajwa TK, SAPPHIRE Investigators. Long-term results of carotid stenting versus endarterectomy in high-risk patients. New England Journal of Medicine 2008;358(15):1572-9.

Yadav J. SAPPHIRE. American Heart Association Scientific Session; 2002 Nov 17-20; Chicago (IL).

Yadav JS. Carotid stenting in high-risk patients: design and rationale of the SAPPHIRE trial. Cleveland Clinical Journal of Medicine 2004;71 Suppl 1:S45-6.

* Yadav JS, Wholey MH, Kuntz RE, Fayad P, Katzen BT, Mishkel GJ, Stenting and Angioplasty with Protection in Patients at High Risk for Endarterectomy Investigators. Protected carotid-artery stenting versus endarterectomy in high-risk patients. New England Journal of Medicine 2004;351(15):1493-501.

\section{SPACE 2006 \{published data only\}}

Eckstein HH, Ringleb P, Allenberg JR, Berger J, Fraedrich G, Hacke W, et al. Results of the Stent-Protected Angioplasty versus Carotid Endarterectomy (SPACE) study to treat symptomatic stenoses at 2 years: a multinational, prospective, randomised trial. Lancet Neurology 2008;7(10):893-902.

Fiehler J, Jansen O, Berger J, Eckstein HH, Ringleb PA, Stingele R. Differences in complication rates among the centres in the SPACE study. Neuroradiology 2008;50(12):1049-53.

Ringleb PA, Kunze A, Allenberg JR, Hennerici MG, Jansen O, Maurer PC, Steering Committee of the SPACE Study. The StentSupported Percutaneous Angioplasty of the Carotid Artery vs Endarterectomy Trial. Cerebrovascular Diseases 2004;18(1):66-8.

* SPACE Collaborative Group. 30 day results from the SPACE trial of stent-protected angioplasty versus carotid endarterectomy in symptomatic patients: a randomised non-inferiority trial. Lancet 2006;368(9543):1239-47.

Witt K, Borsch K, Daniels C, Walluscheck K, Alfke K, Jansen O, et al. Neuropsychological consequences of endarterectomy and endovascular angioplasty with stent placement for treatment of symptomatic carotid stenosis: a prospective randomised study. Journal of Neurology 2007;254(11):1524-32.
SPACE-2 2016 \{published data only\}

* Eckstein HH, Reiff T, Ringleb P, Jansen O, Mansmann U, Hacke W, SPACE Investigators. SPACE-2: a missed opportunity to compare carotid endarterectomy, carotid stenting, and best medical treatment in patients with asymptomatic carotid stenoses. European Journal of Vascular and Endovascular Surgery 2016;51(6):761-5.

Reiff T, Stingele R, Eckstein HH, Fraedrich G, Jansen O, Mudra H, SPACE2-Study Group. Stent-protected angioplasty in asymptomatic carotid artery stenosis vs endarterectomy: SPACE2 - a three-arm randomised-controlled clinical trial. International Journal of Stroke 2009;4(4):294-9.

TESCAS-C 2006 \{published data only\}

TESCAS-C (Ling) study, 2006. www.trialresultscenter.org/ evidenceTable/viewtrial.aspx?trial=8539 (accessed prior to 28 August 2019).

* Ling F, Jiao LQ. Preliminary report of trial of endarterectomy versus stenting for the treatment of carotid atherosclerotic stenosis in China (TESCAS-C). Chinese Journal of Cerebrovascular Diseases 2006;3(1):4-8.

WALLSTENT 2001 \{published and unpublished data\} Alberts MJ. Results of a multicentre prospective randomized trial of carotid artery stenting vs carotid endarterectomy. Stroke 2001;32:325.

* Alberts MJ, McCann R, Smith TP, Stack R, Roubin G, Schneck M, WALLSTENT Endoprosthesis Clinical Investigators. A randomized trial of carotid stenting versus endarterectomy in patients with symptomatic carotid stenosis: study design. Journal of Neurovascular Disease 1997;2(6):228-34.

\section{References to ongoing studies}

\section{ACST-2 \{published data only\}}

Asymptomatic Carotid Surgery Trial (ACST-2) - a large, simple randomised trial to compare carotid endarterectomy versus carotid artery stenting to prevent stroke (complete trial protocol). www.acst.org.uk (last accessed July 2010).

NCT00883402. Carotid endarterectomy versus carotid artery stenting in asymptomatic patients (ACST-2). clinicaltrials.gov/ ct2/show/NCT00883402 (first received 17 April 2009).

* Rudarakanchana N, Dialynas M, Halliday A. Asymptomatic Carotid Surgery Trial-2 (ACST-2): rationale for a randomised clinical trial comparing carotid endarterectomy with carotid artery stenting in patients with asymptomatic carotid artery stenosis. European Journal of Vascular and Endovascular Surgery 2009;38(2):239-42.

\section{Agostoni 2005 \{published data only\}}

Agostoni E, Beghi E, Pappada G, Marina R, Ferrarese C. Early invasive treatment (endarterectomy vs stenting) of moderateto-severe carotid stenosis in patients with transient ischaemic attack or minor stroke. Neurological Sciences 2005;26 Suppl 1:S31-3. 
CREST-2 \{unpublished data only\}

* Howard VJ, Meschia JF, Lal BK, Turan TN, Roubin GS, Brown RD Jr, CREST Investigators. Carotid revascularization and medical management for asymptomatic carotid stenosis: protocol of the CREST-2 clinical trials. International Journal of Stroke 2017;12(7):770-8.

Mott M, Koroshetz W, Wright CB. CREST-2: identifying the best method of stroke prevention for carotid artery stenosis: National Institute of Neurological Disorders and Stroke organizational update. Stroke 2017;48(5):e130-1.

\section{ECST-2 \{published data only\}}

The 2nd European Carotid Surgery Trial (ECST-2); protocol summary. www.ecst2.com (last accessed 12 January 2019).

\section{NCT02841098 \{published data only\}}

NCT02841098. Endarterectomy combined with optimal medical therapy (OMT) vs OMT alone in patients with asymptomatic severe atherosclerotic carotid artery stenosis at higher-than-average risk of ipsilateral stroke (ACTRIS). www.clinicaltrials.gov/ct2/show/NCT02841098 (first received 22 July 2016).

\section{Additional references}

\section{ACAS 1995}

Executive Committee for the Asymptomatic Carotid Atherosclerosis Study. Endarterectomy for asymptomatic carotid artery stenosis. Journal of the American Medical Association 1995;273(18):1421-8.

\section{ACST 2010}

Halliday A, Harrison M, Hayter E, Kong X, Mansfield A, Marro J, Asymptomatic Carotid Surgery Trial (ACST) Collaborative Group. 10-year stroke prevention after successful carotid endarterectomy for asymptomatic stenosis (ACST-1): a multicentre randomised trial. Lancet 2010;376(9746):1074-84.

\section{Alvarez 2012}

Alvarez B, Matas M, Ribo M, Maeso J, Yugueros X, AlvarezSabin J. Transcervical carotid stenting with flow reversal is a safe technique for high-risk patients older than 70 years. Journal of Vascular Surgery 2012;55(4):978-84.

\section{American Stroke Association}

American Stroke Association. WALLSTENT. controlledtrials.com/mrct/trial/WALLSTENT/1026/5243.html.

\section{Anderson 2010}

Anderson HV, Rosenfield KA, White CJ, Ho KK, Spertus JA, Jones PG, et al. Clinical features and outcomes of carotid artery stenting by clinical expert consensus criteria: a report from the CARE registry. Catheter Cardiovascular Interventions 2010;75(4):519-25

\section{Bamford 1989}

Bamford JM, Sandercock PA, Warlow CP, Slattery J. Interobserver agreement for the assessment of handicap in stroke patients. Stroke 1989;20(6):828.

\section{Barbato 2008}

Barbato JE, Dillavou E, Horowitz MB, Jovin TG, Kanal E, David S, et al. A randomized trial of carotid artery stenting with and without cerebral protection. Journal of Vascular Surgery 2008;47(4):760-5.

\section{Bijuklic 2012}

Bijuklic K, Wandler A, Hazizi F, Schofer J. The PROFI study (prevention of cerebral embolization by proximal balloon occlusion compared to filter protection during carotid artery stenting): a prospective randomized trial. Journal of the American College of Cardiology: Cardiovascular Interventions 2012;59(15):1383-9.

\section{Bonaldi 2002}

Bonaldi G. Angioplasty and stenting of the cervical carotid bifurcation: report of a 4 year series. Neuroradiology 2002;44(2):164-74.

\section{Bond 2005}

Bond R, Rerkasem K, Cuffe R, Rothwell PM. A systematic review of the associations between age and sex and the operative risks of carotid endarterectomy. Cerebrovascular Diseases 2005;20(2):69-77.

\section{Bush 2005}

Bush RL, Kougias P, Guerrero MA, Lubbe DF, Zhou W, Lumsden $A B$, et al. A comparison of carotid artery stenting with neuroprotection versus carotid endarterectomy under local anesthesia. American Journal of Surgery 2005;190(5):696-700.

\section{CAPTURE 2007}

Gray WA, Yadav JS, Verta P, Scicli A, Fairman R, Wholey M, CAPTURE Trial Collaborators. The CAPTURE Registry: predictors of outcomes in carotid artery stenting with embolic protection for high surgical risk patients in the early post-approval setting. Catheterization and Cardiovascular Interventions 2007;70(7):1025-33.

\section{CSTC 2010}

Bonati LH, Dobson J, Algra A, Branchereau A, Chatellier G, Fraedrich G, Carotid Stenting Trialists' Collaboration. Shortterm outcome after stenting versus endarterectomy for symptomatic carotid stenosis: a preplanned meta-analysis of individual patient data. Lancet 2010;376(9746):1062-73.

\section{De Weerd 2010}

De Weerd M, Greving JP, Hedblad B, Lorenz MW, Mathiesen EB, O'Leary DH, et al. Prevalence of asymptomatic carotid artery stenosis in the general population: an individual participant data meta-analysis. Stroke 2010;41(6):1294-7.

\section{Diethrich 1996}

Diethrich EB, Ndiaye M, Reid DB. Stenting in the carotid artery. Initial experience in 110 patients. Journal of Endovascular Surgery 1996;3(1):42-62.

\section{ECST 1998}

European Carotid Surgery Trialists Collaboration Group. Randomised trial of endarterectomy for recently symptomatic 
carotid stenosis: final results of the MRC European Carotid Surgery Trial (ECST). Lancet 1998;351(9113):1379-87.

\section{Fischman 1994}

Fischman DL, Leon MB, Baim DS, Schatz RA, Savage MP, Penn I, Stent Restenosis Study Investigators. A randomized comparison of coronary stent placement and balloon angioplasty in the treatment of coronary artery disease. New England Journal of Medicine 1994;331(8):496-501.

\section{Forsting 2007}

Forsting M. Shortcomings and promises of recent carotidstenting trials. Lancet Neurology 2007;6(2):101-2.

\section{Gray 2006}

Gray WA, Hopkins LN, Yadav S, Davis T, Wholey M, Atkinson R, ARCHeR Trial Collaborators. Protected carotid stenting in highsurgical-risk patients: the ARCHeR results. Journal of Vascular Surgery 2006;44(2):258-68.

\section{Guimaraens 2002}

Guimaraens L, Sola MT, Matali A, Arbelaez A, Delgado M, Soler L, et al. Carotid angioplasty with cerebral protection and stenting: report of 164 patients (194 carotid percutaneous transluminal angioplasties). Cerebrovascular Diseases 2002;13(2):114-9.

\section{Higgins 2011}

Higgins JP, Altman DG, Sterne JA. Chapter 8: Assessing risk of bias in included studies. In: Higgins JP, Green S, editor(s). Cochrane Handbook for Systematic Reviews of Interventions Version 5.1.0 (updated March 2011). The Cochrane Collaboration, 2011. Available from handbook.cochrane.org. The Cochrane Collaboration.

\section{Howard 2016}

Howard G, Roubin GS, Jansen O, Hendrikse J, Halliday A, Fraedrich G, Carotid Stenting Trialists' Collaboration. Association between age and risk of stroke or death from carotid endarterectomy and carotid stenting: a meta-analysis of pooled patient data from four randomised trials. Lancet 2016;387(10025):1305-11.

\section{Kastrup 2003}

Kastrup A, Groschel K, Krapf H, Brehm BR, Dichgans J, Schulz JB. Early outcome of carotid angioplasty and stenting with or without cerebral protection devices. A systematic review of the literature. Stroke 2003;34(3):813-9.

\section{Kumar 2017}

Kumar R, Batchelder A, Saratzis A, AbuRahma AF, Ringleb P, Lal BK, et al. Restenosis after carotid interventions and its relationship with recurrent ipsilateral stroke: a systematic review and meta-analysis. European Journal of Vascular and Endovascular Surgery 2017;53(6):766-75.

\section{Leal 2012}

Leal I, Orgaz A, Flores A, Gil J, Rodriguez R, Peinado J, et al. A diffusion-weighted magnetic resonance imaging-based study of transcervical carotid stenting with flow reversal versus transfemoral filter protection. Journal of Vascular Surgery 2012;56(6):1585-90.

\section{Macdonald 2010}

Macdonald S, Evans DH, Griffiths PD, McKevitt FM, Venables GS, Cleveland TJ, et al. Filter-protected versus unprotected carotid artery stenting: a randomised trial. Cerebrovascular Diseases 2010;29(3):282-9.

\section{NASCET 1991}

North American Symptomatic Carotid Endarterectomy Trial Collaborative Group. Beneficial effect of carotid endarterectomy in symptomatic patients with high-grade carotid stenosis. New England Journal of Medicine 1991;325(7):445-53.

\section{NASCET 1998}

Barnett HJ, Taylor DW, Eliasziw M, Fox AJ, Ferguson GG, Haynes RB, North American Symptomatic Carotid Endarterectomy Trial Collaborators. Benefit of carotid endarterectomy in patients with symptomatic moderate or severe stenosis. New England Journal of Medicine 1998;339(20):1415-25.

\section{Park 2013}

Park KY, Kim DI, Kim BM, Nam HS, Kim YD, Heo JH, et al. Incidence of embolism associated with carotid artery stenting: open-cell versus closed-cell stents. Journal of Neurosurgery 2013;119(3):642-7.

\section{Petty 1999}

Petty GW, Brown RD Jr, Whisnant JP, Sicks JD, O'Fallon WM, Wiebers DO. Ischemic stroke subtypes: a population-based study of incidence and risk factors. Stroke 1999;30(12):2513-6.

\section{Reimers 2001}

Reimers B, Corvaja N, Moshari S, Sacca S, Albiero R, Di Mario C, et al. Cerebral protection with filter devices during carotid artery stenting. Circulation 2001;104(1):12-5.

\section{Reimers 2004}

Reimers B, Schluter M, Castriota F, Tubler T, Corvaja N, Cernetti $C$, et al. Routine use of cerebral protection during carotid artery stenting: results of a multicenter registry of 753 patients. American Journal of Medicine 2004;116(4):217-22.

\section{ROADSTER 2015}

Kwolek CJ, Jaff MR, Leal JI, Hopkins LN, Shah RM, Hanover TM, et al. Results of the ROADSTER multicenter trial of transcarotid stenting with dynamic flow reversal. Journal of Vascular Surgery 2015;62(5):1227-34.

\section{Roffi 2010}

Roffi M, Sievert H, Gray WA, White CJ, Torsello G, Cao P, et al. Carotid artery stenting versus surgery: adequate comparisons?. Lancet Neurology 2010;9(4):339-41.

\section{Rothwell 2004}

Rothwell PM, Eliasziw M, Gutnikov SA, Warlow CP, Barnett HJ, Carotid Endarterectomy Trialists Collaboration. Endarterectomy for symptomatic carotid stenosis in relation to clinical subgroups and timing of surgery. Lancet 2004;363(9413):915-24. 


\section{Roubin 2001}

Roubin GS, New G, lyer SS, Vitek JJ, Al-Mubarak N, Liu MW, et al. Immediate and late clinical outcomes of carotid artery stenting in patients with symptomatic and asymptomatic carotid artery stenosis: a 5-year prospective analysis. Circulation 2001;103(4):532-7.

\section{Safian 2006}

Safian RD, Bresnahan JF, Jaff MR, Foster M, Bacharach JM, Maini B, et al. Protected carotid stenting in high-risk patients with severe carotid artery stenosis. Journal of the American College of Cardiology: Cardiovascular Interventions 2006;47(12):2384-9.

\section{Schnaudigel 2008}

Schnaudigel S, Gröschel K, Pilgram SM, Kastrup A. New brain lesions after carotid stenting versus carotid endarterectomy: a systematic review of the literature. Stroke 2008;39(6):1911-9.

\section{Schofer 2015}

Schofer J, Musialek P, Bijuklic K, Kolvenbach R, Trystula M, Siudak Z, et al. A prospective, multicenter study of a novel mesh-covered carotid stent: the CGuard CARENET Trial (Carotid Embolic Protection Using MicroNet). Journal of the American College of Cardiology: Cardiovascular Interventions 2015;8(9):1229-34.

\section{Sedgwick 2014}

Sedgwick P. Relative risks versus odds ratios. $B M J$ 2014;348:g1407.

\section{Serruys 1996}

Serruys PW, de Jaegere P, Kiemeneij F, Macaya C, Rutsch W, Heyndrickx G, Benestent Study Group. A comparison of balloonexpandable-stent implantation with balloon angioplasty in patients with coronary artery disease. New England Journal of Medicine 1996;331(8):489-95.

\section{Stabile 2012}

Stabile E, Garg P, Cremonesi A, Bosiers M, Reimers B, Setacci C, et al. European Registry of Carotid Artery Stenting: results from a prospective registry of eight high volume EUROPEAN institutions. Catheter Cardiovascular Interventions 2012;80(2):329-34.

\section{Stabile 2014}

Stabile E, Sannino A, Schiattarella GG, Gargiulo G, Toscano E, Brevetti $L$, et al. Cerebral embolic lesions detected with diffusion-weighted magnetic resonance imaging following carotid artery stenting: a meta-analysis of 8 studies comparing filter cerebral protection and proximal balloon occlusion. Journal of the American College of Cardiology: Cardiovascular Interventions 2014;7(10):1177-83.

\section{Staubach 2014}

Staubach S, Hein-Rothweiler R, Hochadel M, Segerer M, Zahn R, Jung J, et al. Predictors of minor versus major stroke during carotid artery stenting: results from the carotid artery stenting (CAS) registry of the Arbeitsgemeinschaft Leitende
Kardiologische Krankenhausarzte (ALKK). Clinical Research in Cardiology 2014;103(5):345-51.

\section{Theron 1996}

Theron J, Payelle GG, Coskun O, Huet HF, Guimaraens L. Carotid artery stenosis: treatment with protected balloon angioplasty and stent placement. Neuroradiology 1996;201(3):627-36.

\section{Timaran 2011}

Timaran CH, Rosero EB, Higuera A, Ilarraza A, Modrall JG, Clagett GP. Randomized clinical trial of open-cell vs closed-cell stents for carotid stenting and effects of stent design on cerebral embolization. Journal of Vascular Surgery 2011;54(5):1310-6.

\section{Touzé 2009}

Touzé E, Trinquart L, Chatellier G, Mas JL. Systematic review of the perioperative risks of stroke or death after carotid angioplasty and stenting. Stroke 2009;40(12):e683-93.

\section{Van Swieten 1988}

Van Swieten JC, Koudstaal PJ, Visser MC, Schouten HJ, Van Gijn J. Interobserver agreement for the assessment of handicap in stroke patients. Stroke 1998;19(5):604-7.

\section{White 2006}

White CJ, lyer SS, Hopkins LN, Katzen BT, Russell ME, $\mathrm{BEACH}$ Trial Investigators. Carotid stenting with distal protection in high surgical risk patients: the $\mathrm{BEACH}$ trial 30 day results. Catheterization and Cardiovascular Interventions 2006;67(4):503-12.

\section{References to other published versions of this review \\ Bonati 2012}

Bonati LH, Lyrer P, Ederle J, Featherstone R, Brown MM. Percutaneous transluminal balloon angioplasty and stenting for carotid artery stenosis. Cochrane Database of Systematic Reviews 2012, Issue 9. [DOI: 10.1002/14651858.CD000515]

\section{Coward 2004}

Coward LJ, Featherstone RL, Brown MM. Percutaneous transluminal angioplasty and stenting for carotid artery stenosis. Cochrane Database of Systematic Reviews 2004, Issue 2. [DOI: 10.1002/14651858.CD000515.pub2]

\section{Crawley 1997}

Crawley F, Brown MM. Percutaneous transluminal angioplasty and stenting for carotid artery stenosis. Cochrane Database of Systematic Reviews 1997, Issue 4. [DOI: 10.1002/14651858.CD000515.pub2]

\section{Ederle 2007}

Ederle J, Featherstone R, Brown MM. Percutaneous transluminal angioplasty and stenting for carotid artery stenosis. Cochrane Database of Systematic Reviews 2007, Issue 4. [DOI: 10.1002/14651858.CD000515.pub3]

* Indicates the major publication for the study 


\title{
CHARACTERISTICS OF STUDIES
}

\section{Characteristics of included studies [author-defined order]}

Leicester 1998

Methods Single-centre randomised trial (Leicester Royal Infirmary, UK)

Planned sample size 300 participants; 23 participants randomly assigned to endovascular treatment ( $\mathrm{n}$ $=11)$ or endarterectomy $(n=12)$ between June and September 1996.

\begin{abstract}
Participants
Inclusion criteria: symptomatic carotid stenosis $\geq 70 \%$ measured with duplex ultrasound (no restrictions as of time of most recent event reported; events dated back to 10 months prior to randomisation among included participants)
\end{abstract}

Exclusion criteria: asymptomatic stenosis, symptomatic 0-69\% stenosis, crescendo TIA or stroke in evolution, and vertebrobasilar or non-hemispheric symptoms

Mean age of study participants 67 years, 53\% men (of those receiving the randomly allocated treatment)

Mean degree of stenosis $82 \%$

Endovascular treatment: predilation with balloon-angioplasty where necessary, insertion of a stent (WALLSTENT, Schneider, USA), and postdilation in all cases. No protection devices were available at the time the trial was conducted. A consultant radiologist with personal experience of $>4000$ angioplasties to peripheral arteries, who had also done 8 successful procedures in the carotid artery before the trial, performed the procedures.

Endarterectomy: performed under general anaesthesia, with routine use of shunting and patching. 1 consultant surgeon or 1 supervised trainee performed the procedures.

Aspirin therapy was "not stopped" before treatment.

All participants received iv heparin at the time of the procedure.

All participants were monitored with transcranial Doppler and received an iv infusion of dextran in case of sustained microembolisation.

Outcomes

Primary method of comparison: per-protocol, including only participants receiving the allocated treatment

Primary outcome: death or any stroke within 30 days of treatment

Secondary outcomes relevant for this review included separate reporting of primary outcome components, disabling and non-disabling strokes, as well as cranial nerve palsies.

Disabling stroke was defined as a stroke leading to a score of 3-6 on the Oxfordshire Handicap Stroke scale 30 days after treatment.

\begin{tabular}{ll}
\hline Follow-up & 24 hours and 30 days after treatment \\
\hline Funding & The Stroke Association, UK \\
& Stents were provided at cost price by Schneider UK Ltd
\end{tabular}

Trial registration number No registration found

Notes

Terminated early by recommendation of the data monitoring committee due to safety concerns.

\section{Risk of bias}


Leicester 1998 (Continued)

\section{Bias Authors' judgement Support for judgement}

Random sequence genera- Low risk tion (selection bias)
Quote: "Three hundred random treatment methods were numbered and sealed in opaque envelopes and allocated on a consecutive basis ..."

Comment: adequate

Allocation concealment Low risk
(selection bias)

risk

Quote: "Three hundred random treatment methods were numbered and
sealed in opaque envelopes and allocated on a consecutive basis ..."

Comment: adequate

Blinding of participants Low risk
and personnel (perfor-
mance bias)
All outcomes

Comment: blinding not possible, but the outcome was unlikely to have been influenced.

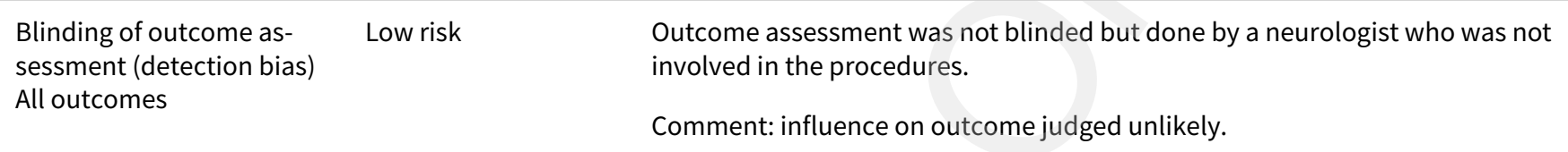

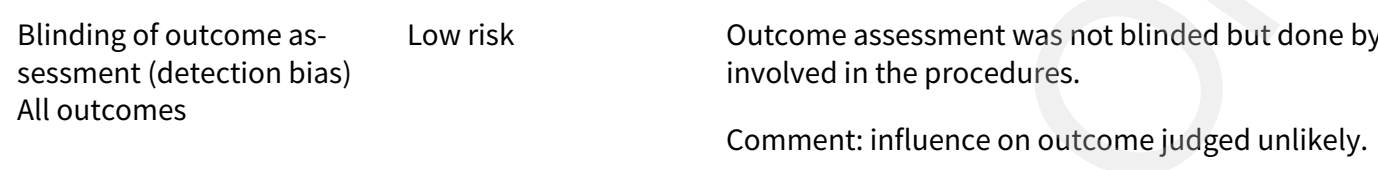

Incomplete outcome data Unclear risk

(attrition bias)

All outcomes

\begin{abstract}
4 participants randomised to endovascular treatment (1 participant refusing the allocated treatment after admission and 3 participants waiting for admission when the trial was suspended) and 2 participants randomised to surgery (1 participant refusing the allocated treatment and 1 participant occluding the relevant ICA) did not receive their allocated treatment and were excluded from analysis.

Comment: outcome events occurring after randomisation were not systematically reported for these participants. In particular, it was unclear whether the participants refusing the allocated treatment crossed over to receive the alternative treatment.
\end{abstract}

$\begin{array}{ll}\begin{array}{l}\text { Selective reporting (re- } \\ \text { porting bias) }\end{array} & \text { Low risk } \\ & \text { Comment: no reference to a study protocol was made, but the generally ex- } \\ \text { pected major outcome events were reported. }\end{array}$

\section{CAVATAS-CEA 2001}

$\begin{array}{ll}\text { Methods } & \text { International randomised multicentre trial (22 centres in Europe, Australia, and Canada) } \\ & 505 \text { participants randomly assigned to endovascular treatment }(\mathrm{n}=252) \text { or endarterectomy }(\mathrm{n}=253) \\ \text { between March } 1992 \text { and July } 1997\end{array}$

Participants

Inclusion criteria: symptomatic or asymptomatic carotid artery stenosis $\geq 30 \%$ according to the common carotid artery method, documented by intra-arterial angiography or consistent findings $\geq 2$ noninvasive imaging methods, considered to require revascularisation and suitable for surgery and endovascular treatment

Exclusion criteria: unsuitable for surgery because of medical or surgical risk factors, unwillingness or inability to give informed consent, unwillingness to undergo either procedure, thrombus present on preliminary angiography, intracranial stenosis beyond the skull base, major stroke with no useful recovery of function within the region supplied by the treatable artery
$90 \%$ of participants $(n=452)$ had ischaemic symptoms in the territory of the relevant carotid artery in the 6 months before randomisation.

Mean age of study participants 68 years, $70 \%$ men 
CAVATAS-CEA 2001 (Continued)

Mean degree of carotid stenosis $86 \%$ (common carotid artery method)

Interventions $\quad$ Endovascular treatment: balloon angioplasty with or without stent insertion. Stents were used in 55 participants ( $26 \%$ of successful endovascular procedures) and included WALLSTENT (Schneider, USA), Streker (Medi-Tech, USA), and Palmaz (Johnson and Johnson, USA). No protection devices were available at the time the trial was conducted. Participants received a minimum of aspirin $150 \mathrm{mg}$ daily or an alternative antiplatelet agent for $\geq 24$ hours prior to the procedure and throughout the trial, as well as heparin during the procedure and for the following 24 hours. Prior expertise in endovascular treatment was required, albeit not in the carotid artery, as this was considered an entirely new treatment for carotid stenosis in most participating centres at the time, and not defined by a minimum number of procedures.

Endarterectomy: using technique routinely used by the collaborating surgeon (no specification on type of anaesthesia, shunting or patching, heparin or antiplatelet therapy during the procedure). Surgical expertise in CEA was requested but not defined by a minimum number of procedures.

All participants received BMT throughout the study, which was specified to include antiplatelet therapy (or anticoagulant, as appropriate), and control of hypertension, diabetes, and hypercholesterolaemia.

Outcomes

Primary method of comparison: intention-to-treat

Primary safety outcome: disabling stroke or death within 30 days of treatment

Secondary safety outcomes relevant for this review included the following events occurring within 30 days of treatment: death, disabling stroke, any stroke, MI, cranial nerve palsy, haematoma requiring surgery, or extending hospital stay

Primary efficacy outcome: disabling stroke or death between randomisation and end of follow-up

Secondary efficacy outcomes relevant for this review included any stroke, ipsilateral stroke, and restenosis measured on ultrasound, occurring during follow-up

Follow-up

1 and 6 months after treatment, then 1 year after randomisation and annually thereafter, clinically and with carotid ultrasound

No predefined maximum length of follow-up; centres were encouraged to follow up participants for as long as the centre and individual participants were willing to do so.

$\begin{array}{ll}\text { Funding } & \text { British Heart Foundation } \\ \text { National Health Service Management Executive, UK } \\ \text { The Stroke Association, UK }\end{array}$

Trial registration number

ISRCTN 01425573

\section{Notes}

\section{Risk of bias}

Bias Authors' judgement Support for judgement

Random sequence genera- Low risk tion (selection bias)
Quote: "patients were randomly assigned to a treatment group, in equal proportions, by telephone call or fax to the randomisation centre at the Clinical Trial Service Unit in Oxford, UK, ... We randomly assigned patients by computer with a minimisation algorithm, which took account of centre and timing of symptoms, ..."

Comment: adequate

\begin{tabular}{|c|c|c|}
\hline $\begin{array}{l}\text { Allocation concealment } \\
\text { (selection bias) }\end{array}$ & Low risk & $\begin{array}{l}\text { Quote: "patients were randomly assigned to a treatment group, in equal pro- } \\
\text { portions, by telephone call or fax to the randomisation centre at the Clinical }\end{array}$ \\
\hline
\end{tabular}

Carotid artery stenting versus endarterectomy for treatment of carotid artery stenosis (Review)

Copyright $\odot 2020$ The Cochrane Collaboration. Published by John Wiley \& Sons, Ltd. 
Trial Service Unit in Oxford, UK, ... We randomly assigned patients by computer with a minimisation algorithm, which took account of centre and timing of symptoms, ..."

Comment: adequate

\begin{tabular}{|c|c|c|}
\hline $\begin{array}{l}\text { Blinding of participants } \\
\text { and personnel (perfor- }\end{array}$ & Low risk & $\begin{array}{l}\text { Comment: blinding not possible, but the outcome was unlikely to have been } \\
\text { influenced. }\end{array}$ \\
\hline
\end{tabular}
mance bias)

All outcomes

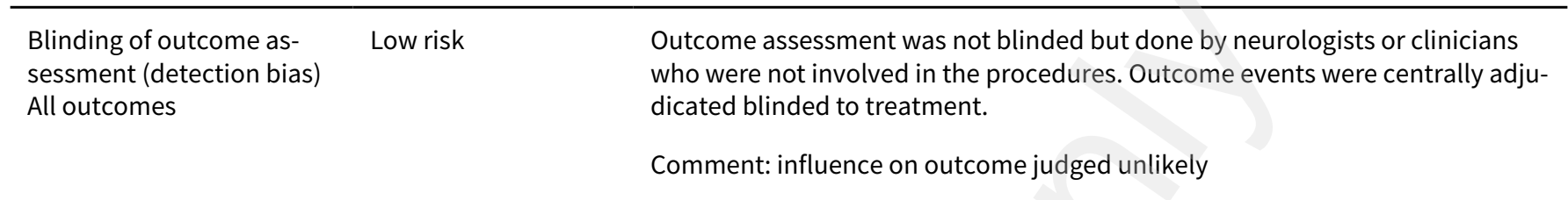

Incomplete outcome data Low risk

1 participant with carotid occlusion assigned endovascular treatment was ran-

(attrition bias) domised in error and excluded from the analysis. Numbers of participants at

All outcomes risk during follow-up provided.

Comment: censoring assumed to be non-informative.

Selective reporting (re- Low risk Comment: the primary analysis prespecified in the protocol was reported.
porting bias)

\section{Kentucky 2001}

\begin{tabular}{ll}
\hline Methods & Single-centre randomised trial (Central Baptist Hospital, Lexington, KT, USA) \\
& 104 participants randomised to endovascular treatment $(n=53)$ or endarterectomy $(n=51)$
\end{tabular}

\section{Participants}

Inclusion criteria: symptomatic carotid stenosis $>70 \%$ according to NASCET criteria documented by intra-arterial angiography (most recent event in the previous 3 months), anticipated life expectancy 5 years, willingness to complete treatment within 2 weeks

Exclusion criteria: NIHSS > 4, cardiac arrhythmia, allergy/sensitivity to aspirin, other antiplatelets or heparin, coagulopathy, recent intracranial haemorrhage, vertebrobasilar insufficiency, intracranial occlusive disease

Mean age of study participants 68 years, no information on sex distribution of participants provided Mean degree of stenosis $85 \%$ postdilation by balloon-angioplasty. No distal protection devices were used.

Endarterectomy: performed under general anaesthesia with standard operative techniques, using intraoperative EEG monitoring (no further details provided).

No information on prior experience of interventionalists or surgeons provided.

Participants in both arms received aspirin $325 \mathrm{mg}$ and clopidogrel $75 \mathrm{mg}$ before the procedure, and participants in the endovascular group received heparin at the time of the procedure.

Outcomes No primary outcome was defined. Reported outcome events relevant for this review included death, stroke, cranial nerve palsy, and haematoma requiring treatment. 
Kentucky 2001 (Continued)

Funding Stents were provided by Boston Scientific, USA

Trial registration number No registration found

Notes

\section{Risk of bias}

Bias Authors' judgement Support for judgement

Random sequence genera- Unclear risk tion (selection bias)

Quote: "... two-arm randomized clinical trial ...". "... were selected randomly for CEA or carotid stenting ..."

Comment: no information on method of randomisation provided.

Allocation concealment Unclear risk Comment: no information provided
(selection bias)

$\begin{array}{ll}\begin{array}{l}\text { Blinding of participants } \\ \text { and personnel (perfor- }\end{array} & \text { Low risk }\end{array}$

mance bias)

All outcomes

\begin{tabular}{|c|c|c|}
\hline \multirow{2}{*}{$\begin{array}{l}\text { Blinding of outcome as- } \\
\text { sessment (detection bias) } \\
\text { All outcomes }\end{array}$} & Low risk & $\begin{array}{l}\text { Outcome assessment was not blinded but done by a neurologist who was not } \\
\text { involved in the procedures. }\end{array}$ \\
\hline & & Comment: influence on outcome judged unlikely. \\
\hline
\end{tabular}

\begin{tabular}{lll}
\hline $\begin{array}{l}\text { Incomplete outcome data } \\
\text { (attrition bias) } \\
\text { All outcomes }\end{array}$ & Unclear risk & $\begin{array}{l}\text { Numbers of participants at risk during follow-up not provided. } \\
\text { Comment: bias possible }\end{array}$ \\
\hline $\begin{array}{l}\text { Selective reporting (re- } \\
\text { porting bias) }\end{array}$ & Low risk & $\begin{array}{l}\text { Comment: no reference to a study protocol was made but the generally ex- } \\
\text { pected major outcome events were reported. }\end{array}$ \\
\hline
\end{tabular}

\section{WALLSTENT 2001}

\begin{tabular}{ll}
\hline Methods & Multicentre randomised trial (USA) \\
& $\begin{array}{l}\text { Planned sample size } 700 \text { participants; } 219 \text { participants randomly assigned to endovascular treatment } \\
(\mathrm{n}=107) \text { or endarterectomy }(\mathrm{n}=112)\end{array}$
\end{tabular}

\begin{tabular}{|c|c|}
\hline \multirow[t]{3}{*}{ Participants } & $\begin{array}{l}\text { Inclusion criteria: symptomatic carotid stenosis } \geq 60 \% \text { documented by intra-arterial angiography (most } \\
\text { recent event in the last } 120 \text { days), aged }>18 \text { years, medically stable, life expectancy } \geq 2 \text { years } \\
\text { Exclusion criteria: ipsilateral arterial stenosis greater than the target lesion (i.e. tandem stenosis), } \\
\text { NIHSS score } \geq 15 \text {, Rankin score }>2 \text {, Barthel score } \leq 60 \text {, atrial fibrillation, left ventricular thrombus, endo- } \\
\text { carditis, heparin sensitivity, not suitable for surgery, moderate or severe dementia, bleeding diathesis } \\
\text { or coagulopathy, history of intracranial haemorrhage }\end{array}$ \\
\hline & Mean age of study participants 68 years, $64 \%$ men \\
\hline & Mean degree of stenosis $76 \%$ \\
\hline Interventions & $\begin{array}{l}\text { Endovascular treatment: insertion of the WALLSTENT endoprosthesis (Schneider, USA), performed } \\
\text { within } 2 \text { weeks of randomisation. Predilation and postdilation was done routinely. No protection de- } \\
\text { vices were used. Participants received aspirin } 325 \mathrm{mg} \text { bd and ticlopidine } 250 \mathrm{mg} \text { bd for } 3 \text { days prior to }\end{array}$ \\
\hline
\end{tabular}


WALLSTENT 2001 (Continued)

treatment and heparin during treatment. Following treatment, all participants received aspirin and ticlopidine for 4 weeks and then aspirin $325 \mathrm{mg}$ bd only. Interventionalists had to have performed $\geq 10$ stent procedures in the carotid artery using the WALLSTENT endoprosthesis with 30-day complication rates of $\leq 10 \%$.

Endarterectomy: must have been performed within 2 weeks of randomisation. Surgical technique and type of anaesthesia were not specified. Participants were treated with aspirin $325 \mathrm{mg}$ bd following the procedure for the duration of the study. Use of ticlopidine was optional. Surgeons had to have performed $\geq 30$ carotid endarterectomies in the previous 2 years with 30 -day death or stroke rates of $\leq 6 \%$.

Primary method of comparison: unknown
Primary outcome: any death within 30 days of treatment, or ipsilateral stroke or vascular death withi
year of treatment
Secondary outcomes relevant for this review included any death, major stroke, MI, and target vess
patency during follow-up
Disabling stroke was defined as a stroke leading to a Barthel score of $<90$, an NIHSS score $\geq 5$, or a
Rankin score $>2$.

Follow-up 24 hours; and 1, 6, and 12 months after treatment and then annually clinically

1,6 , and 12 months after treatment and then annually by carotid ultrasound

\begin{tabular}{ll}
\hline Funding & Schneider, USA \\
\hline Trial registration number & No registration found \\
\hline Notes & Terminated early by the sponsor after randomisation due to safety and futility concerns.
\end{tabular}

\section{Risk of bias}

\begin{tabular}{lll}
\hline Bias & Authors' judgement & Support for judgement \\
\hline $\begin{array}{ll}\text { Random sequence genera- } \\
\text { tion (selection bias) }\end{array}$ & Low risk & $\begin{array}{l}\text { Quote: "Randomization of patients is performed using a computerized random } \\
\text { number generator. Assignment is provided in sequentially numbered, sealed } \\
\text { envelopes." } \\
\text { Comment: adequate }\end{array}$ \\
\end{tabular}

\begin{tabular}{ll}
\hline $\begin{array}{l}\text { Allocation concealment } \\
\text { (selection bias) }\end{array}$ & Low risk \\
& $\begin{array}{l}\text { Quote: "Randomization of patients is performed using a computerized random } \\
\text { number generator. Assignment is provided in sequentially numbered, sealed } \\
\text { envelopes." }\end{array}$ \\
& Comment: adequate \\
\hline
\end{tabular}

\begin{tabular}{ll}
\hline $\begin{array}{l}\text { Blinding of participants } \\
\text { and personnel (perfor- } \\
\text { mance bias) }\end{array}$ & $\begin{array}{l}\text { Comment: blinding not possible, but the outcome was unlikely to have been } \\
\text { influenced. }\end{array}$ \\
All outcomes &
\end{tabular}

\begin{tabular}{lll}
\hline $\begin{array}{l}\text { Blinding of outcome as- } \\
\text { sessment (detection bias) } \\
\text { All outcomes }\end{array}$ & Low risk & $\begin{array}{l}\text { Outcome assessment was not blinded but done by study neurologists who } \\
\text { were not involved in the procedures. }\end{array}$ \\
\hline $\begin{array}{l}\text { Incomplete outcome data } \\
\text { (attrition bias) }\end{array}$ & Unclear risk & $\begin{array}{l}\text { Trial results presented at the } 2001 \text { International Stroke Conference and pub- } \\
\text { All outcomes }\end{array}$
\end{tabular}


Comment: bias possible

$\begin{array}{ll}\text { Methods } & \text { Single-centre randomised trial (Xuanwu Hospital, Capital Medical University, Beijing, China) } \\ & 21 \text { participants randomised to endovascular treatment }(n=8) \text { or medical care alone }(n=13) \text { between } \\ & 2001 \text { and } 2003\end{array}$

Participants Inclusion criteria: carotid stenosis $>70 \%$ and contralateral carotid occlusion, demonstrated on duplex ultrasound or intra-arterial angiography

Exclusion criteria: cardiorespiratory dysfunction or plasma glucose $>8 \mathrm{mmol} / \mathrm{L}$ despite medication

Mean age 69 years, $71 \%$ men. All participants were described as having had symptoms of TIA or stroke before randomisation but no maximum time between last symptoms and randomisation was specified.

Mean degree of stenosis was $83 \%$. devices was provided. Participants received iv heparin during treatment, and ticlopidine $250 \mathrm{mg}$ daily for 6 weeks and aspirin $300 \mathrm{mg}$ daily for 6 months after treatment, followed by aspirin $100 \mathrm{mg}$ daily for life

Medical care: individual risk factor targeting

\begin{tabular}{ll}
\hline Outcomes & No primary method of comparison defined \\
& $\begin{array}{l}\text { No primary outcome was specified. Numbers of participants with death, stroke, or TIA during follow-up } \\
\text { were reported. }\end{array}$
\end{tabular}

Follow-up Single follow-up 1.5 years after treatment was specified, assessing recurrent events, and carotid steno-
sis on duplex ultrasound.

\begin{tabular}{ll}
\hline Funding & No information available \\
\hline Trial registration number & No registration found
\end{tabular}

\begin{tabular}{lll}
\hline Notes & \\
\hline Risk of bias & \\
\hline Bias & Authors' judgement & Support for judgement \\
\hline $\begin{array}{l}\text { Random sequence genera- } \\
\text { tion (selection bias) }\end{array}$ & Low risk & Quote: "Patients were allocated to two groups by random number table." \\
\hline $\begin{array}{l}\text { Allocation concealment } \\
\text { (selection bias) }\end{array}$ & Low risk & Comment: adequate \\
\hline
\end{tabular}


Beijing 2003 (Continued)

Blinding of participants Low risk Comment: blinding not possible, but the outcome was unlikely to have been and personnel (perforinfluenced.

mance bias)

All outcomes

Blinding of outcome as-
sessment (detection bias) $\quad$ Unclear risk Comment: no information available

All outcomes

Incomplete outcome data Low risk

Comment: outcome events for all participants were provided.

(attrition bias)

All outcomes

$\begin{array}{ll}\begin{array}{l}\text { Selective reporting (re- } \\ \text { porting bias) }\end{array} & \text { Low risk }\end{array}$

\section{Kentucky 2004}

Methods Single-centre randomised trial (Central Baptist Hospital, Lexington, KY, USA)

85 participants randomised to endovascular treatment $(n=43)$ or endarterectomy $(n=42)$ within 1 year

Participants Inclusion criteria: asymptomatic carotid stenosis $>80 \%$ according to NASCET criteria documented by intra-arterial angiography, anticipated life expectancy 5 years, willingness to complete treatment within 1 month

Exclusion criteria: any symptoms of cerebrovascular ischaemia

Mean age of study participants 68 years, no information on sex distribution of participants provided

Mean degree of stenosis $86 \%$

\begin{tabular}{ll}
\hline Interventions & Identical to Kentucky 2001 trial \\
\hline Outcomes & See Kentucky 2001 trial \\
\hline Follow-up & 24 hours; and 1, 3, 6, 12, 24, and 48 months after treatment, clinically and with carotid ultrasound \\
\hline Funding & No financial support \\
\hline Trial registration number & No registration found
\end{tabular}

Notes

\begin{tabular}{lll}
\hline Bias & Authors' judgement & Support for judgement \\
\hline $\begin{array}{ll}\text { Random sequence genera- } \\
\text { tion (selection bias) }\end{array}$ & Unclear risk & $\begin{array}{l}\text { Quotes: "... two-arm randomized clinical trial ...". "... were selected randomly } \\
\text { to undergo CEA ... or CAS ..." }\end{array}$ \\
& & Comment: no information on method of randomisation provided. \\
\hline
\end{tabular}

\begin{tabular}{lll}
\hline $\begin{array}{l}\text { Allocation concealment } \\
\text { (selection bias) }\end{array}$ & Unclear risk & Comment: no information provided. \\
\hline
\end{tabular}


Kentucky 2004 (Continued)

Blinding of participants Low risk Comment: blinding not possible, but the outcome was unlikely to have been and personnel (perforinfluenced.

mance bias)

All outcomes

Blinding of outcome as-
sessment (detection bias)

All outcomes

Outcome assessment was not blinded but done by a neurologist who was not involved in the procedures.

Comment: influence on outcome judged unlikely.

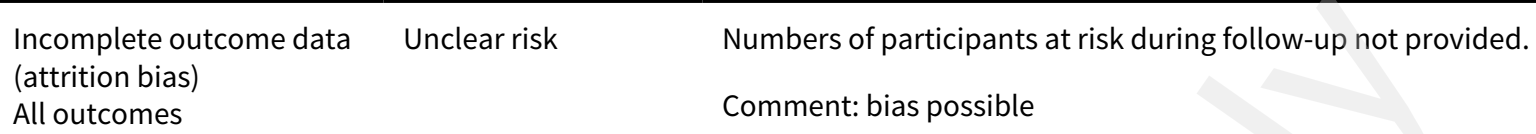

$\begin{array}{ll}\begin{array}{l}\text { Selective reporting (re- } \\ \text { porting bias) }\end{array} & \text { Low risk } \\ \text { pected major outcome events were reported. }\end{array}$

Methods Multicentre randomised non-inferiority trial (29 centres in the USA)

No predefined sample size; recruitment was planned to stop if non-inferiority of stenting was demonstrated at repeated interim analyses. 334 participants randomly assigned to endovascular treatment or endarterectomy ( $n=167$ in each arm) between August 2000 and July 2002.

\section{Participants}

Inclusion criteria: aged $\geq 18$ years, symptomatic stenosis $\geq 50 \%$ or asymptomatic stenosis $\geq 80 \%$ according to NASCET criteria documented by duplex ultrasound; high surgical risk defined by $\geq 1$ of the following criteria: significant cardiopulmonary disease, contralateral carotid occlusion, contralateral laryngeal nerve palsy, previous radical neck surgery or radiotherapy to the neck, recurrent stenosis after endarterectomy, aged $>80$ years

Exclusion criteria: ischaemic stroke within the previous 48 hours, intraluminal thrombus, vascular disease precluding endovascular treatment, intracranial aneurysm, need for $>2$ stents, history of bleeding disorder, planned percutaneous or surgical intervention, life expectancy $<1$ year, ostial lesion in the common carotid or brachiocephalic artery

Mean age 72.5 years, $67 \%$ men. $71 \%$ had asymptomatic carotid stenosis.

Interventions

Endovascular treatment: stent insertion (Smart or Precise, Cordis, Johnson \& Johnson, USA) with routine use of a distal protection device (Angioguard or Angioguard XP, Cordis, Johnson \& Johnson, USA). Participants received aspirin $81 \mathrm{mg}$ or $325 \mathrm{mg}$ daily starting $\geq 72$ hours before stenting and indefinitely thereafter, plus clopidogrel $75 \mathrm{mg}$ daily starting 24 hours before stenting until 2-4 weeks after treatment. Interventionalists had performed a median of 64 carotid stent procedures before joining the trial (range 20-700).

Endarterectomy: performed according to customary techniques. Participants received aspirin $81 \mathrm{mg}$ or $325 \mathrm{mg}$ daily starting $\geq 72$ hours before endarterectomy and indefinitely thereafter. Surgeons had median annual volumes of 30 carotid endarterectomies (range 15-100) and had to demonstrate periprocedural stroke or death rates of $<6 \%$ prior to joining the trial.

All participants were given heparin during the procedure.

Outcomes

Method of comparison: intention-to-treat and per-protocol

Non-inferiority margin (primary safety outcome or its 30-day components): 95\% confidence interval of risk difference below 3.0\% (type 1 error probability 1-sided 2.5\%)

Primary outcome: death, any stroke, or MI within 30 days after treatment or death or ipsilateral stroke between 31 days and 1 year after treatment 
SAPPHIRE 2004 (Continued)

Secondary outcomes relevant for this review included components of the primary outcome, major and minor stroke, cranial nerve palsy, and death or any stroke within 30 days after treatment, or ipsilateral stroke or death from neurological causes up to 3 years after treatment

Follow-up 24 hours; 30 days; and 6 and 12 months after treatment, and annually thereafter for 3 years clinically

Duplex ultrasound before discharge and at each visit except 30 days after treatment

\begin{tabular}{ll}
\hline Funding & Cordis, Johnson \& Johnson, USA \\
\hline Trial registration number & NCT00231270 \\
\hline Notes & Terminated early due to a drop in the randomisation rate.
\end{tabular}

\section{Risk of bias}

\begin{tabular}{lll}
\hline Bias & Authors' judgement & Support for judgement \\
\hline $\begin{array}{l}\text { Random sequence genera- } \\
\text { tion (selection bias) }\end{array}$ & Low risk & $\begin{array}{l}\text { Quote: "Randomization was performed with the use of a pseudo random-num- } \\
\text { ber generator, and the numbers were distributed by an automated, central- } \\
\text { ized telephone response system." }\end{array}$ \\
\hline $\begin{array}{l}\text { Allocation concealment } \\
\text { (selection bias) }\end{array}$ & Low risk & $\begin{array}{l}\text { Comment: adequate } \\
\text { ber generator, and the numbers were distributed by an automated, central- } \\
\text { ized telephone response system." }\end{array}$ \\
& $\begin{array}{l}\text { Comment: adequate }\end{array}$
\end{tabular}

$\begin{array}{ll}\begin{array}{l}\text { Blinding of participants } \\ \text { and personnel (perfor- }\end{array} & \text { Low risk } \\ \text { manfluenced. }\end{array}$

mance bias)

All outcomes

Blinding of outcome as-
sessment (detection bias) All outcomes

Low risk

Outcome assessment was not blinded but done by neurologists who were not involved in the procedures. Outcome events were centrally adjudicated blinded to treatment.

Comment: influence on outcome judged unlikely.

\section{Incomplete outcome data Low risk} (attrition bias)

$143 / 167$ (86\%) participants in the endovascular arm and 117/167 (70\%) partici-

All outcomes pants in the surgical arm had available follow-up at 3 years.

Numbers of participants at risk during follow-up provided.

Comment: censoring assumed to be non-informative

\begin{tabular}{ll}
\hline $\begin{array}{l}\text { Selective reporting (re- } \\
\text { porting bias) }\end{array}$ & Unclear risk \\
& $\begin{array}{l}\text { Protocol was published only a few months before the initial results of the trial. } \\
\text { Expected major outcome events were reported, but not for symptomatic and } \\
\text { asmptomatic participants separately. }\end{array}$ \\
$\begin{array}{l}\text { Concern has been expressed because the Chief Investigator of SAPPHIRE re- } \\
\text { ceived undeclared royalties from sales of the protection device used in the tri- } \\
\text { al. }\end{array}$ \\
Comment: bias possible
\end{tabular}


EVA-3S 2006

Multicentre randomised non-inferiority trial (30 centres in France)
Planned sample size 872 participants; 527 participants randomly assigned to endovascular treatment
$(\mathrm{n}=265)$ or endarterectomy $(\mathrm{n}=262)$ between November 2000 and September 2005

Participants

Inclusion criteria: aged $\geq 18$ years, symptomatic carotid stenosis of $60-99 \%$ according to NASCET criteria documented by intra-arterial angiography or a combination of duplex ultrasound and magnetic resonance angiography (most recent event in the last 120 days before randomisation)

Exclusion criteria: modified Rankin score $\geq 3$; non-atherosclerotic carotid disease; severe tandem lesion; previous revascularisation of the relevant carotid artery; history of bleeding disorder; uncontrolled hypertension or diabetes; unstable angina; contraindication to heparin, ticlopidine, or clopidogrel; life expectancy < 2 years; or percutaneous or surgical intervention within 30 days before or after the study procedure

Mean age 70 years, $75 \%$ men

$93 \%$ of participants had $\geq 70 \%$ degree of stenosis

Interventions

Endovascular treatment: stent insertion. Use of protection devices became mandatory in the stenting group during the course of the study. Interventionalists had to have performed $\geq 12$ carotid stenting procedures or $\geq 35$ stenting procedures in the supra-aortic trunks, of which $\geq 5$ were in the carotid artery.

Endarterectomy: vascular surgeons performed endarterectomies by their routinely used techniques. Surgeons had to have performed $\geq 25$ endarterectomies in the year before enrolment.

It was recommended to use aspirin 100-300 mg daily in all participants, and clopidogrel $75 \mathrm{mg}$ or ticlopidine $500 \mathrm{mg}$ daily for 3 days before and 30 days after stenting.

Outcomes

Primary method of comparison: modified intention-to-treat analysis for periprocedural outcomes, including only randomised participants who underwent carotid revascularisation, analysed by the randomly assigned treatment. Regular intention-to-treat analysis for long-term outcomes

Non-inferiority margin (primary safety outcome): $90 \%$ confidence interval of risk difference $<2.0 \%$ (type 1 error probability 1-sided 5\%)

Primary safety outcome: any stroke or death within 30 days after treatment

Secondary safety outcomes relevant to this review included primary outcome components, disabling stroke, MI, cranial nerve palsy, and access site haematoma

Primary efficacy outcome: any stroke or death within 30 days of treatment or ipsilateral stroke up to 4 years after treatment

Secondary efficacy outcomes relevant to this review included any stroke, and death or any stroke up to 4 years after treatment, and carotid restenosis up to 3 years after treatment

Follow-up $\quad 48$ hours, 1 month, and then every 6 months after treatment for 2-4 years clinically

$1,6,12,18,24$, and 36 months after randomisation with duplex ultrasound

Funding French Ministry of Health

Trial registration number NCT00190398

Notes

Terminated early by recommendation of the safety committee due to safety and futility concerns.

\section{Risk of bias}


EVA-3S 2006 (Continued)

\section{Bias Authors' judgement Support for judgement}

Random sequence genera- Low risk tion (selection bias)
Quote: "Randomization was carried out centrally by means of a computer-generated sequence, involving randomized blocks of two, four, or six patients that were stratified according to study center and degree of stenosis ..."

Comment: adequate
Quote: "Randomization was carried out centrally by means of a computer-generated sequence, involving randomized blocks of two, four, or six patients that were stratified according to study center and degree of stenosis ..."

Comment: adequate

\begin{tabular}{|c|c|c|}
\hline $\begin{array}{l}\text { Blinding of participants } \\
\text { and personnel (perfor- }\end{array}$ & Low risk & $\begin{array}{l}\text { Comment: blinding not possible, but the outcome was unlikely to have been } \\
\text { influenced. }\end{array}$ \\
\hline
\end{tabular}
influenced.

mance bias)

All outcomes

\section{Blinding of outcome as- Low risk sessment (detection bias)} All outcomes
Outcome assessment was not blinded but done by neurologists who were not involved in the procedures. Outcome events were centrally adjudicated blinded to treatment.

Comment: influence on outcome judged unlikely.

$\begin{array}{ll}\begin{array}{l}\text { Incomplete outcome data } \\ \text { (attrition bias) }\end{array} & \text { Low risk } \\ \text { All but } 3 \text { participants were followed up to death or end of study. Numbers of } \\ \text { participants at risk during follow-up provided. }\end{array}$

All outcomes

Comment: censoring assumed to be non-informative.

Selective reporting (re- Low risk Comment: the primary analysis prespecified in the protocol was reported.
porting bias)

\section{SPACE 2006}

Methods
Multicentre, randomised non-inferiority trial (35 centres in Germany, Austria, and Switzerland)

Planned sample size 1900 participants, revised upwards to 2500 after interim analysis; 1214 participants randomly assigned to endovascular treatment $(n=613)$ or endarterectomy $(n=601)$ between March 2001 and February 2006.

A single centre substudy enrolled 48 participants with the aim of evaluating cognitive performance after CEA vs endovascular treatment. No predefined sample size documented for the substudy.

Participants
Inclusion criteria: symptomatic carotid stenosis $\geq 50 \%$ according to NASCET criteria or $\geq 70 \%$ according to ECST criteria, documented by duplex ultrasound (most recent event in the last 180 days before randomisation), aged $>50$ years, modified Rankin score $\leq 3$

Exclusion criteria: intracranial bleeding in the previous 90 days; uncontrolled hypertension; intracranial arteriovenous malformation or aneurysm; life expectancy < 2 years; coagulation abnormality; contraindications for heparin, aspirin, or clopidogrel; planned surgery; stenosis due to dissection, irradiation, or occurring after previous revascularisation; floating thrombus; intracranial tandem stenosis of higher degree

Mean age 69 years, $72 \%$ men

$62 \%$ of participants had $\geq 70 \%$ degree of stenosis 
SPACE 2006 (Continued)

Interventions
Endovascular treatment: stent insertion. Choice of balloon size, predilation, and use of protection devices were at the discretion of the interventionalist. Used stents and protection devices had to be CEcertified and approved for use in the study by a separate committee. Participants had to be given aspirin $100 \mathrm{mg}$ plus clopidogrel $75 \mathrm{mg}$ daily for 3 days before and 30 days after treatment. Interventionalists had to show proof $\geq 25$ consecutive successful angioplasty or stent procedures in the carotid artery.

Carotid endarterectomies: performed by vascular surgeons who needed to have 25 consecutive procedures completed. Surgeons used their routine techniques without specifications on type of anaesthesia or shunt use. Participants had to be given aspirin $\geq 100 \mathrm{mg}$ before, during, and after surgery.

\section{Outcomes}

Primary method of comparison: intention-to-treat

Non-inferiority margin (primary safety outcome): $90 \%$ confidence interval of risk difference below $2.5 \%$ (type 1 error probability 1-sided 5\%)

Primary safety outcome: death or ipsilateral stroke between randomisation and 30 days after treatment

Secondary safety outcomes relevant for this review included death or any stroke, death or disabling stroke, death, and any stroke between randomisation and 30 days after treatment

Primary efficacy outcome: death or any stroke between randomisation and 30 days after treatment, or ipsilateral stroke up to 2 years after randomisation

Secondary efficacy outcomes relevant for this review included death or any stroke, and carotid restenosis within 2 years after randomisation

A substudy investigated cognitive performance at 6 and 30 days after treatment. Primary outcome of this substudy was any difference in neuropsychological changes after endarterectomy vs endovascular therapy

Follow-up

1,7 , and 30 days after treatment, and 6, 12, and 24 months after randomisation clinically and with duplex ultrasound

6 and 30 days after treatment with neuropsychological evaluation at a single centre substudy

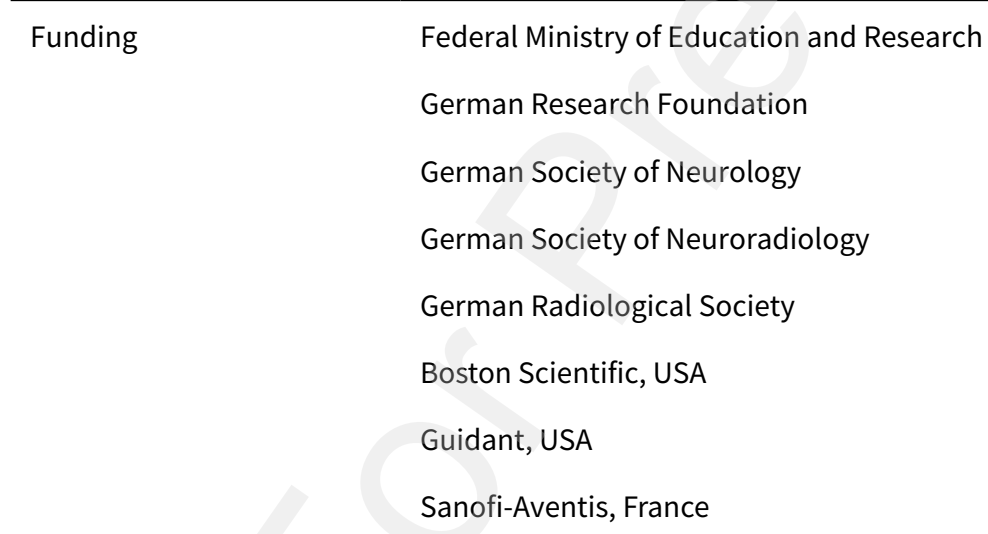

Trial registration number ISRCTN57874028

Notes Terminated early by the steering committee due to futility concerns and lack of funding.

\section{Risk of bias}

Bias Authors' judgement Support for judgement


SPACE 2006 (Continued)

Random sequence genera- Low risk tion (selection bias)
Quote: "The random allocation schedule was generated using a computer program. This was done by members of the data and statistic centre, who also obtained and analysed the data."

Comment: adequate

\section{Allocation concealment Low risk} (selection bias)
Quote: "The random allocation schedule was generated using a computer program. This was done by members of the data and statistic centre, who also obtained and analysed the data."

Comment: adequate

$\begin{array}{ll}\begin{array}{l}\text { Blinding of participants } \\ \text { and personnel (perfor- }\end{array} & \text { Low risk } \\ \text { mance } & \begin{array}{l}\text { Comment: blinding not possible, but the outcome was unlikely to have been } \\ \text { influenced. }\end{array}\end{array}$
and personnel (perforinfluenced.

mance bias)

All outcomes

Blinding of outcome as- Low risk sessment (detection bias) All outcomes
Outcome assessment was not blinded but done by neurologists who were not involved in the procedures. Outcome events were centrally adjudicated blinded to treatment.

Comment: influence on outcome judged unlikely.

\section{Incomplete outcome data Low risk} (attrition bias)

All outcomes
6 participants in endovascular arm and 12 participants in surgical arm immediately withdrew consent after randomisation

541 (89\%) participants in the endovascular arm and 522 (89\%) participants in the surgical arm had follow-up data available at 2 years. Reasons for attrition provided. Numbers of participants at risk during follow-up provided.

Comment: no information on clinical outcome was available from participants who withdrew consent and were excluded from the analysis in the trial; however, the numbers were small and the risk of bias was considered low. Censoring assumed to be non-informative.

Selective reporting (re- Low risk porting bias)
Comment: the primary analysis prespecified in the protocol was reported.

\section{TESCAS-C 2006}

\begin{tabular}{ll}
\hline Methods & Multicentre, randomised trial (China) \\
& 166 participants randomly assigned to endovascular treatment $(\mathrm{n}=82)$ or endarterectomy ( $=84)$ \\
\hline Participants & Inclusion criteria: symptomatic carotid stenosis $>50 \%$ or asymptomatic carotid stenosis $>70 \%$ \\
& Mean age was 63 years, no information on sex distribution of participants available. \\
\hline Interventions & Endovascular treatment: stent insertion with routine use of protection devices \\
\hline Outcomes & Primary outcome: death, stroke, or Ml at 30 within days after treatment; or death or ipsilateral stroke \\
\hline bollow-up & 1 and 6 months after treatment \\
\hline
\end{tabular}


TESCAS-C 2006 (Continued)

\begin{tabular}{ll} 
Funding & No information available \\
\hline Trial registration number & No registration found \\
\hline Notes & $\begin{array}{l}\text { Publication in Chinese with English abstract only. Data presented in review taken from abstract and the } \\
\text { following website: www.trialresultscenter.org/study8539-TESCAS-C- }\end{array}$
\end{tabular}

\section{Risk of bias}

\begin{tabular}{lll}
\hline Bias & Authors' judgement & Support for judgement \\
\hline $\begin{array}{l}\text { Random sequence genera- } \\
\text { tion (selection bias) }\end{array}$ & Unclear risk & Quote: "... a multicentre randomized controlled trial ..." \\
& & Comment: method of randomisation not known. \\
\hline $\begin{array}{l}\text { Allocation concealment } \\
\text { (selection bias) }\end{array}$ & Unclear risk & Comment: method of randomisation not known. \\
\hline $\begin{array}{l}\text { Blinding of participants } \\
\begin{array}{l}\text { and personnel (perfor- } \\
\text { mance bias) }\end{array}\end{array}$ & Low risk & $\begin{array}{l}\text { Comment: blinding not possible, but the outcome was unlikely to have been } \\
\text { All outcomes }\end{array}$ \\
\hline
\end{tabular}

\begin{tabular}{|c|c|c|}
\hline $\begin{array}{l}\text { Blinding of outcome as- } \\
\text { sessment (detection bias) } \\
\text { All outcomes }\end{array}$ & Unclear risk & Comment: no information available \\
\hline $\begin{array}{l}\text { Incomplete outcome data } \\
\text { (attrition bias) } \\
\text { All outcomes }\end{array}$ & Unclear risk & Comment: no information available \\
\hline $\begin{array}{l}\text { Selective reporting (re- } \\
\text { porting bias) }\end{array}$ & Low risk & $\begin{array}{l}\text { Comment: no reference to a study protocol was made but the generally ex- } \\
\text { pected major outcome events were reported. }\end{array}$ \\
\hline
\end{tabular}

\section{BACASS 2008}

Single-centre randomised trial (University Hospital Basel, Switzerland)
No sample size was calculated; 20 participants were randomly assigned to endovascular treatment $(\mathrm{n}=$
10) or endarterectomy $(\mathrm{n}=10)$ between November 1998 and February 2002.

\section{Participants}

Inclusion criteria: symptomatic carotid stenosis $\geq 70 \%$ according to NASCET criteria, documented by concordant findings on duplex ultrasound and magnetic resonance angiography, or by intra-arterial angiography (all included participants had their most recent event in the last 3 months before randomisation)

Exclusion criteria: unavailable for $\geq 2$ years for follow-up, carotid occlusion or free-floating thrombus, carotid stenosis occurring after prior revascularisation or neck irradiation, intracranial haemorrhage within 2 months prior to treatment, intracranial mass lesions or vascular malformations, life expectancy $<2$ years

Mean age 70 years, $85 \%$ men

Mean degree of stenosis $83 \%$ 
routine postdilation. Dual antiplatelet therapy with aspirin and clopidogrel was started prior to, or immediately after, the procedure and continued for 1 month thereafter.

Endarterectomy: under general anaesthesia with intraoperative EEG monitoring or local anaesthesia, with or without patch use, with selective shunting, and under single antiplatelet therapy.

Previous centre experience included approximately 50 carotid endarterectomies and 15 carotid stent procedures annually.

\begin{tabular}{ll}
\hline Outcomes & $\begin{array}{l}\text { 3 separate primary safety outcomes were defined: stroke, death, and MI within } 30 \text { days after treatment. } \\
\text { Secondary outcomes relevant for this review included major stroke (defined by an increase in NIHSS by } \\
\text { 4 points, or symptoms including aphasia or hemianopia for }>24 \text { hours), access site haematoma and } \\
\text { cranial nerve palsy occurring within } 30 \text { days after treatment, and ipsilateral stroke and restenosis dur- } \\
\text { ing follow-up. }\end{array}$ \\
\hline Follow-up & 1 day; 1,6, and 12 months after treatment; and yearly thereafter, clinically and with ultrasound \\
\hline Funding & No financial support \\
\hline Trial registration number & No registration found \\
\hline Notes & Recruitment was stopped when the centre joined the International Carotid Stenting Study (ICSS 2010).
\end{tabular}

\section{Risk of bias}

\begin{tabular}{lll}
\hline Bias & Authors' judgement & Support for judgement \\
\hline $\begin{array}{l}\text { Random sequence genera- } \\
\text { tion (selection bias) }\end{array}$ & Low risk & $\begin{array}{l}\text { Quote: "As randomisation procedure sealed envelopes were used for treat- } \\
\text { ment allocation." } 50 \text { envelopes were prepared. } \\
\text { Comment: adequate }\end{array}$ \\
\hline $\begin{array}{l}\text { Allocation concealment } \\
\text { (selection bias) }\end{array}$ & Low risk & $\begin{array}{l}\text { Quote: "As randomisation procedure sealed envelopes were used for treat- } \\
\text { ment allocation." 50 envelopes were prepared. }\end{array}$ \\
& Comment: adequate
\end{tabular}

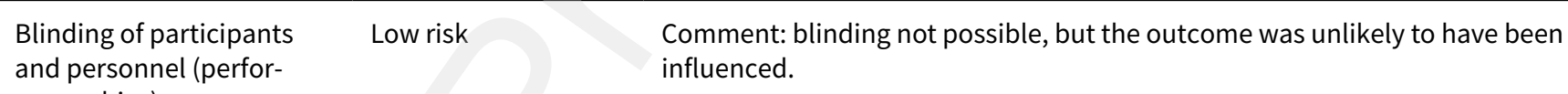
mance bias)

All outcomes

\begin{tabular}{|c|c|c|}
\hline $\begin{array}{l}\text { Blinding of outcome as- } \\
\text { sessment (detection bias) } \\
\text { All outcomes }\end{array}$ & Low risk & $\begin{array}{l}\text { Outcome assessment was not blinded but done by neurologists who were not } \\
\text { involved in the procedures. } \\
\text { Comment: influence on outcome judged unlikely. }\end{array}$ \\
\hline $\begin{array}{l}\text { Incomplete outcome data } \\
\text { (attrition bias) } \\
\text { All outcomes }\end{array}$ & Low risk & $\begin{array}{l}\text { Comment: numbers of participants at risk during follow-up and reasons for } \\
\text { loss of follow-up provided. }\end{array}$ \\
\hline $\begin{array}{l}\text { Selective reporting (re- } \\
\text { porting bias) }\end{array}$ & Low risk & $\begin{array}{l}\text { Comment: no reference to a study protocol was made but the generally ex- } \\
\text { pected major outcome events were reported. }\end{array}$ \\
\hline
\end{tabular}

Methods Single-centre randomised trial (University Hospital Regensburg, Germany)


Regensburg 2008 (Continued)

Planned sample size 200 participants; 87 participants randomised to endovascular treatment $(n=43)$ or endarterectomy $(n=44)$ between August 1999 and April 2002

\section{Participants}

Inclusion criteria: aged $40-80$ years, symptomatic carotid stenosis $\geq 70 \%$ according to NASCET criteria documented by intra-arterial angiography

Exclusion criteria: contralateral carotid stenosis $\geq 70 \%$ (symptomatic) or $\geq 90 \%$ (asymptomatic), tandem stenosis, cerebral ischaemia within the previous 4 weeks, floating thrombus, dissection, near-occlusion, fibromuscular dysplasia, previous endarterectomy in the relevant artery

Mean age 69 years, no sex distribution provided

Mean degree of stenosis $85 \%$
Endovascular treatment: insertion of a single type of stent (WALLSTENT, Boston Scientific, USA), with routine postdilation and no use of protection devices. A single experienced radiologist performed the procedures (number of pretrial procedures not provided).

Endarterectomy: performed under regional anaesthesia with eversion technique and selective shunting.

Participants in both arms received clopidogrel $300 \mathrm{mg}$ and aspirin $100 \mathrm{mg}$ on the day before treatment and heparin during treatment, followed by clopidogrel $75 \mathrm{mg}$ and aspirin $100 \mathrm{mg}$ daily for 30 days, and aspirin $300 \mathrm{mg}$ daily thereafter. In addition, participants in the stenting group received aspirin $500 \mathrm{mg}$ iv before treatment. Primary outcome specified in the protocol: cerebral ischaemia (including stroke or TIA), MI, or death within 30 days of treatment

Prespecified secondary outcomes relevant for this review included ipsilateral cerebral ischaemia or death, and severe carotid restenosis or occlusion during follow-up

The prospectively defined follow-up 12 months included clinical examinations and carotid ultrasound
3-5 days, 30 days, 6 and 12 months after treatment
Long-term follow-up assessed retrospectively up to a median of 66 months in the endovascular arm
and 64 months in the surgical arm

\begin{tabular}{ll}
\hline Funding & $\begin{array}{l}\text { Bristol Meyer Squibb, USA } \\
\text { Boston Scientific, USA }\end{array}$ \\
\hline Trial registration number & No registration found \\
\hline Notes & $\begin{array}{l}\text { Recruitment was stopped after the Stent-Protected Angioplasty Versus Carotid Endarterectomy (SPACE } \\
\text { 2006) trial was initiated. }\end{array}$
\end{tabular}

\section{Risk of bias}

Bias Authors' judgement Support for judgement

Random sequence genera- Low risk tion (selection bias)
Quote [translation from German]: "200 patients will be randomised using a random key, half into the surgical arm and half into the interventional arm. Stratification will be done according to age ..., degree of stenosis ..., and presence of coronary heart disease."

Comment: adequate

\begin{tabular}{|c|c|c|}
\hline $\begin{array}{l}\text { Allocation concealment } \\
\text { (selection bias) }\end{array}$ & Low risk & $\begin{array}{l}\text { Quote [translation from German]: "200 patients will be randomised using a ran- } \\
\text { dom key, half into the surgical arm and half into the interventional arm. Strat- }\end{array}$ \\
\hline
\end{tabular}


ification will be done according to age ..., degree of stenosis ..., and presence of coronary heart disease."

Comment: adequate

$\begin{array}{ll}\begin{array}{l}\text { Blinding of participants } \\ \text { and personnel (perfor- }\end{array} & \text { Low risk } \\ \text { influenced. }\end{array}$

mance bias)

All outcomes

\section{Blinding of outcome as- Low risk sessment (detection bias)}

All outcomes
Outcome assessment was not blinded but done by neurologists who were not involved in the procedures.

Comment: influence on outcome judged unlikely

\section{Beijing 2009}

\begin{tabular}{|c|c|}
\hline Methods & $\begin{array}{l}\text { Single-centre randomised trial (Peking Union Medical College Hospital, Beijing, China) } \\
40 \text { participants were randomised to stenting ( } 23 \text { procedures) or endarterectomy ( } 23 \text { procedures; } 3 \text { par- } \\
\text { ticipants in each arm were treated bilaterally) between May } 2004 \text { and December } 2006\end{array}$ \\
\hline Participants & Inclusion criteria: symptomatic carotid stenosis $\geq 50 \%$ or asymptomatic carotid stenosis $\geq 70 \%$ \\
\hline Interventions & $\begin{array}{l}\text { Endovascular treatment: stent insertion. Protection devices were used in } 21 / 23 \text { procedures } \\
\text { Endarterectomy } \\
\text { Shunts were used in } 9 \text { and patches in } 12 / 23 \text { endarterectomies } \\
\text { No further information available }\end{array}$ \\
\hline Outcomes & $\begin{array}{l}\text { Primary outcome specified in the abstract: any stroke or death within } 30 \text { days after treatment } \\
\text { Secondary outcomes relevant to this review included periprocedural MI, wound haematoma; and ipsi- } \\
\text { lateral stroke and carotid restenosis occurring up to } 18 \text { months after treatment }\end{array}$ \\
\hline Follow-up & 1 and 18 months after treatment \\
\hline Funding & No information available \\
\hline Trial registration number & No registration found \\
\hline Notes & Publication in Chinese with English abstract only. Results were taken from abstract. \\
\hline
\end{tabular}


Beijing 2009 (Continued)

Risk of bias

\begin{tabular}{|c|c|c|}
\hline Bias & Authors' judgement & Support for judgement \\
\hline $\begin{array}{l}\text { Random sequence genera- } \\
\text { tion (selection bias) }\end{array}$ & Unclear risk & $\begin{array}{l}\text { Quote: "A prospective randomised single-center clinical trial ..." } \\
\text { Comment: method of randomisation unknown }\end{array}$ \\
\hline $\begin{array}{l}\text { Allocation concealment } \\
\text { (selection bias) }\end{array}$ & Unclear risk & $\begin{array}{l}\text { Quote: "A prospective randomised single-center clinical trial ..." } \\
\text { Comment: method of randomisation unknown }\end{array}$ \\
\hline $\begin{array}{l}\text { Blinding of participants } \\
\text { and personnel (perfor- } \\
\text { mance bias) } \\
\text { All outcomes }\end{array}$ & Low risk & $\begin{array}{l}\text { Comment: blinding not possible, but the outcome was unlikely to have been } \\
\text { influenced. }\end{array}$ \\
\hline $\begin{array}{l}\text { Blinding of outcome as- } \\
\text { sessment (detection bias) } \\
\text { All outcomes }\end{array}$ & Unclear risk & Comment: no information available \\
\hline $\begin{array}{l}\text { Incomplete outcome data } \\
\text { (attrition bias) } \\
\text { All outcomes }\end{array}$ & Unclear risk & Comment: no information available \\
\hline $\begin{array}{l}\text { Selective reporting (re- } \\
\text { porting bias) }\end{array}$ & Low risk & $\begin{array}{l}\text { Comment: no reference to a study protocol was made but the generally ex- } \\
\text { pected major outcome events were reported. }\end{array}$ \\
\hline
\end{tabular}

\section{CAVATAS-MED 2009}

\begin{tabular}{ll}
\hline Methods & International randomised multicentre trial (12 centres in Europe, Australia, and Canada) \\
& 40 participants randomly assigned to endovascular treatment in combination with BMT $(\mathrm{n}=20)$ or BMT \\
& alone $(\mathrm{n}=20)$ between April 1992 and May 1997
\end{tabular}

\begin{tabular}{ll}
\hline Participants & Inclusion criteria: symptomatic or asymptomatic carotid stenosis warranting treatment (see CA- \\
VATAS-CEA 2001), unsuitable for surgery because of surgical or medical contraindications \\
Exclusion criteria: see CAVATAS-CEA 2001 \\
$62.5 \%$ of participants $(\mathrm{n}=25)$ had cerebrovascular symptoms in the previous 6 months before randomi- \\
sation. \\
Mean age of study participants 69 years, $78 \%$ men \\
Mean degree of carotid stenosis $86 \%$ (common carotid artery method) \\
\hline Interventions \\
$\begin{array}{l}\text { Endovascular treatment: see CAVATAS-CEA 2001 } \\
\text { of the individual centres. }\end{array}$ \\
\hline Primary method of comparison: intention-to-treat \\
Primary outcome measure: stroke or death during follow-up \\
\hline Follow-up \\
$\begin{array}{l}\text { Identical to CAVATAS-CEA 2001. The first follow-up visit in the medical arm was done } 1 \text { month after ran- } \\
\text { domisation. }\end{array}$
\end{tabular}


CAVATAS-MED 2009 (Continued)

\begin{tabular}{ll} 
Funding & See CAVATAS-CEA 2001 \\
\hline Trial registration number & ISRCTN 01425573 \\
\hline
\end{tabular}

Notes

\section{Risk of bias}

\begin{tabular}{|c|c|c|}
\hline Bias & Authors' judgement & Support for judgement \\
\hline $\begin{array}{l}\text { Random sequence genera- } \\
\text { tion (selection bias) }\end{array}$ & Low risk & $\begin{array}{l}\text { Quote: see CAVATAS-CEA } 2001 \\
\text { Comment: adequate }\end{array}$ \\
\hline $\begin{array}{l}\text { Allocation concealment } \\
\text { (selection bias) }\end{array}$ & Low risk & $\begin{array}{l}\text { Quote: see CAVATAS-CEA } 2001 \\
\text { Comment: adequate }\end{array}$ \\
\hline $\begin{array}{l}\text { Blinding of participants } \\
\text { and personnel (perfor- } \\
\text { mance bias) } \\
\text { All outcomes }\end{array}$ & Low risk & $\begin{array}{l}\text { Comment: blinding not possible, but the outcome was not likely to have been } \\
\text { influenced. }\end{array}$ \\
\hline $\begin{array}{l}\text { Blinding of outcome as- } \\
\text { sessment (detection bias) } \\
\text { All outcomes }\end{array}$ & Low risk & $\begin{array}{l}\text { Outcome assessment was not blinded but done by neurologists who were not } \\
\text { involved in the procedures. Outcome events were centrally adjudicated blind- } \\
\text { ed to treatment. } \\
\text { Comment: influence on outcome judged unlikely }\end{array}$ \\
\hline $\begin{array}{l}\text { Incomplete outcome data } \\
\text { (attrition bias) } \\
\text { All outcomes }\end{array}$ & Low risk & $\begin{array}{l}\text { Numbers of participants at risk during follow-up provided. } \\
\text { Comment: censoring assumed to be non-informative. }\end{array}$ \\
\hline $\begin{array}{l}\text { Selective reporting (re- } \\
\text { porting bias) }\end{array}$ & Low risk & Comment: the primary analysis prespecified in the protocol was reported. \\
\hline
\end{tabular}

\section{CREST 2010}

Methods Multicentre randomised trial (108 centres in the USA and 9 centres in Canada)

Both superiority and non-inferiority analyses were specified in the protocol.

Calculated sample size 2500 participants; 2522 participants with symptomatic or asymptomatic carotid stenosis randomly assigned to carotid stenting $(n=1271)$ or endarterectomy $(n=1251)$ between December 2000 and July 2008.

\section{Participants}

Inclusion criteria: aged $\geq 18$ years, symptomatic carotid stenosis $\geq 50 \%$ on intra-arterial angiography or $\geq 70 \%$ on duplex ultrasound or non-invasive angiography (most recent event within 180 days before randomisation); or asymptomatic carotid stenosis of 60-99\% on intra-arterial angiography, $70-99 \%$ on duplex ultrasound, or 80-99\% on non-invasive angiography

Exclusion criteria: major or evolving stroke, atrial fibrillation, $\mathrm{MI}$ in the previous 30 days, unstable angina, bleeding history, dementia, increased surgical risk, postirradiation or postendarterectomy stenosis

Mean age 69 years, $65 \%$ men

$86 \%$ of participants had $\geq 70 \%$ degree of stenosis 
CREST 2010 (Continued)

Interventions
Carotid stenting: insertion of the RX Acculink stent and, whenever feasible, use of the RX Accunet protection device (Abbott Vascular Solutions, USA). Participants received aspirin $650 \mathrm{mg}$ plus clopidogrel $150 \mathrm{mg}$ daily $\geq 48$ hours before treatment, or aspirin $650 \mathrm{mg}$ plus clopidogrel $450 \mathrm{mg} \geq 4$ hours before treatment, and aspirin 325-650 mg daily plus either clopidogrel $75 \mathrm{mg}$ or ticlopidine $500 \mathrm{mg}$ daily for 28-30 days after treatment. Interventionists with $<30$ carotid stent procedures were required to complete a specific training programme. All Interventionists were certified to participate based on evaluation of their performance during a mean of 20 procedures in a lead-in phase.

Endarterectomy: technique was not specified. Participants received aspirin $325 \mathrm{mg}$ daily $\geq 48$ hours before treatment. Surgeons had to show performance $>12$ procedures annually with rates of complications or death $<3 \%$ in asymptomatic and $<5 \%$ in symptomatic participants.

Primary outcome: death, stroke, or MI between randomisation and 30 days after treatment, or ipsilateral stroke up to 4 years after randomisation

Secondary outcomes relevant for this review included periprocedural components of the primary outcome, major stroke defined "on the basis of clinical data or if the NIHSS score was 9 or higher 90 days after the procedure," cranial nerve palsy not resolving for $\geq 1$ month, access site haematoma requiring treatment, and degree of stenosis of the relevant carotid artery 6 and 12 months after treatment

Follow-up 18-54 hours and 1 month after treatment, and every 6 months thereafter up to 4 years clinically, and by telephone interviews between visits

Functional assessment at each visit included the NIHSS and the modified Rankin scale.

Carotid ultrasound was performed 1, 6, and 12 months after treatment and annually thereafter.

Cardiac enzyme levels were measured before and 6-8 hours after treatment.

ECG was performed before stenting or endarterectomy, as well as 6-48 hours and 1 month thereafter.

Funding
National Institute of Neurological Disorders and Stroke
National Institutes of Health
Abbott Vascular (formerly Guidant), USA

Trial registration number NCT00004732

Notes

Inclusion criteria were changed in 2005 to include participants with asymptomatic stenosis.

\section{Risk of bias}

Bias Authors' judgement Support for judgement

$\begin{array}{ll}\begin{array}{l}\text { Random sequence genera- } \\ \text { tion (selection bias) }\end{array} & \text { Low risk } \\ & \begin{array}{l}\text { Quote: "Eligible patients were randomly assigned, with the use of a Web-based } \\ \text { system, to undergo either carotid-artery stenting or carotid endarterectomy. } \\ \text { Randomization was based on a permuted-block design (with random block } \\ \text { sizes of } 2,4, \text { or 6), was stratified according to center and symptomatic status } \\ \ldots . "\end{array} \\ & \text { Comment: adequate }\end{array}$

\begin{tabular}{ll}
\hline $\begin{array}{l}\text { Allocation concealment } \\
\text { (selection bias) }\end{array}$ & Quote: "Eligible patients were randomly assigned, with the use of a Web-based \\
& system, to undergo either carotid-artery stenting or carotid endarterectomy. \\
& Randomization was based on a permuted-block design (with random block \\
& sizes of 2,4, or 6$),$ was stratified according to center and symptomatic status \\
& $\ldots$.
\end{tabular}




$\begin{array}{ll}\begin{array}{l}\text { Blinding of participants } \\ \text { and personnel (perfor- }\end{array} & \text { Low risk } \\ \text { influenced. }\end{array}$

mance bias)

All outcomes

\begin{tabular}{|c|c|c|}
\hline \multirow[t]{2}{*}{$\begin{array}{l}\text { Blinding of outcome as- } \\
\text { sessment (detection bias) } \\
\text { All outcomes }\end{array}$} & Low risk & $\begin{array}{l}\text { Outcome assessment was not blinded but done by clinicians who were not in- } \\
\text { volved in the procedures. Outcome events were adjudicated centrally, blinded } \\
\text { to treatment. }\end{array}$ \\
\hline & & Comment: influence on outcome judged unlikely \\
\hline
\end{tabular}

Incomplete outcome data Low risk (attrition bias)

All outcomes

\begin{abstract}
9 participants in the endovascular group and 11 participants in the endarterectomy group enrolled at a single centre were excluded from the analysis due to scientific misconduct. 33 participants in the endovascular arm and 47 participants in the endarterectomy arm who were included in the analysis were lost to follow-up during the trial. Numbers of participants at risk during follow-up provided.

Comment: no information on clinical outcome was available from excluded participants; however, the numbers were small and the risk of bias was considered low. Numbers of participants lost to follow-up were considered low. Censoring was assumed to be non-informative.
\end{abstract}

Selective reporting (re- Low risk Comment: the primary analysis prespecified in the protocol was reported.
porting bias)
porting bias) assigned to carotid stenting $(n=855)$ or endarterectomy $(n=858)$ between May 2001 and October 2008, 1511 of which at experienced centres

A substudy evaluating cognitive performance at 2 centres in the Netherlands enrolled 177 participants. The original target sample size for the substudy was 200 participants, but the study was terminated early when enrolment in the main ICSS trial was stopped.

Participants

Inclusion criteria: symptomatic atheromatous carotid stenosis $\geq 50 \%$ according to NASCET criteria documented by non-invasive imaging (including duplex ultrasound) or intra-arterial angiography (most recent event in the previous 12 months), aged $>40$ years

Exclusion criteria: major stroke without useful recovery of function, previous endarterectomy or stenting in the relevant carotid artery, planned major surgery

Mean age 70 years, $70 \%$ men

$90 \%$ of participants had $\geq 70 \%$ degree of stenosis.

Interventions

Carotid stenting: insertion of CE-certified stents. Use of protection devices was recommended but not mandatory. A combination of aspirin and clopidogrel to cover stent procedures was recommended, the use of heparin mandatory.

Endarterectomy: technique, type of anaesthesia, and use of shunts and patches were left to the discretion of the surgeons. 
ICSS 2010 (Continued)

Centres were enrolled by a credentialing committee based on previous experience and classified as experienced (requiring a surgeon who had done $\geq 50$ carotid endarterectomies and an interventionalist who had done $\geq 50$ stent procedures, 10 of which in the carotid artery) or supervised (in centres with less experience, procedures were proctored by an outside surgeon, or interventionalist).

Primary method of comparison: intention-to-treat
Primary safety outcome: death, any stroke, or Ml occurring within 120 days after randomisation
Secondary safety outcomes relevant to this review included components of the primary outcome, dis-
abling stroke (defined by a score on the modified Rankin scale $\geq 3,30$ days after stroke onset), cranial
nerve palsy, and access site haematoma requiring surgery or prolonging hospital stay
Primary efficacy outcome specified in the protocol: long-term rate of fatal or disabling stroke in any ter-
ritory
The primary outcome of the cognition substudy was the change in cognitive sum z score between base-
line and follow-up. Secondary outcome measures included changes in individual cognitive domain
scores. Domains included attention, abstract reasoning, executive functioning, language, verbal mem-
ory, visual memory, visual perception, neglect.

Follow-up $\quad 30$ days after treatment, 6 and 12 months after randomisation, and annually thereafter, clinically and with ultrasound

6 months after treatment for the cognition substudy

\begin{tabular}{|c|c|c|}
\hline Funding & \multicolumn{2}{|c|}{ Medical Research Council } \\
\hline & \multicolumn{2}{|l|}{ The Stroke Association } \\
\hline & \multicolumn{2}{|l|}{ European Union } \\
\hline & \multicolumn{2}{|c|}{ Sanofi-Synthélabo, France } \\
\hline Trial registration number & ISRCTN2533747 & \\
\hline \multicolumn{3}{|l|}{ Notes } \\
\hline \multicolumn{3}{|l|}{ Risk of bias } \\
\hline Bias & Authors' judgement & Support for judgement \\
\hline \multirow[t]{2}{*}{$\begin{array}{l}\text { Random sequence genera- } \\
\text { tion (selection bias) }\end{array}$} & Low risk & $\begin{array}{l}\text { Quote: "Eligible patients were randomly assigned in a 1:1 ratio to receive } \\
\text { carotid artery stenting or carotid endarterectomy by use of a computerised } \\
\text { service provided by Oxford Clinical Trials Service Unit staff who were not in- } \\
\text { volved in other parts of the trial. ... Randomisation was stratified by centre } \\
\text { with minimisation for sex, age, contralateral occlusion, and side of the ran- } \\
\text { domised artery." }\end{array}$ \\
\hline & & Comment: adequate \\
\hline \multirow[t]{2}{*}{$\begin{array}{l}\text { Allocation concealment } \\
\text { (selection bias) }\end{array}$} & Low risk & $\begin{array}{l}\text { Quote: "Eligible patients were randomly assigned in a 1:1 ratio to receive } \\
\text { carotid artery stenting or carotid endarterectomy by use of a computerised } \\
\text { service provided by Oxford Clinical Trials Service Unit staff who were not in- } \\
\text { volved in other parts of the trial. ... Randomisation was stratified by centre } \\
\text { with minimisation for sex, age, contralateral occlusion, and side of the ran- } \\
\text { domised artery." }\end{array}$ \\
\hline & & Comment: adequate \\
\hline
\end{tabular}




\section{ICSS 2010 (Continued)}

Blinding of participants Low risk Comment: blinding not possible, but the outcome was unlikely to have been and personnel (performance bias)

All outcomes

\section{Blinding of outcome as- Low risk sessment (detection bias)} All outcomes

\begin{tabular}{|c|c|c|}
\hline $\begin{array}{l}\text { Incomplete outcome data } \\
\text { (attrition bias) } \\
\text { All outcomes }\end{array}$ & Low risk & $\begin{array}{l}2 \text { participants in the endovascular arm and } 1 \text { participant in the surgical arm } \\
\text { immediately withdrew consent after randomisation. } \\
\text { Comment: no information on clinical outcome was available from these partic } \\
\text { ipants, who were excluded from the analysis in the trial; however, the numbers } \\
\text { were small and the risk of bias was considered low. Numbers of participants } \\
\text { at risk during follow-up were provided; censoring assumed to be non-informa- } \\
\text { tive. }\end{array}$ \\
\hline $\begin{array}{l}\text { Selective reporting (re- } \\
\text { porting bias) }\end{array}$ & Low risk & $\begin{array}{l}\text { Comment: the primary analysis prespecified in the protocol for the compari- } \\
\text { son of long-term outcome had not been performed at the time of writing, but } \\
\text { secondary short-term analyses specified in the protocol have been reported. }\end{array}$ \\
\hline
\end{tabular}

\begin{abstract}
Outcome assessment was not blinded but done by clinicians who were not involved in the procedures. Outcome events were adjudicated centrally, as well as by an independent external adjudicator, blinded to treatment.
\end{abstract}

Comment: influence on outcome judged unlikely

\section{Beijing 2013}

\begin{tabular}{ll} 
Methods & Multicentre randomised trial ( 2 centres in China) \\
& $\begin{array}{l}\text { No predefined sample size documented, } 63 \text { participants randomly assigned to endovascular treatment } \\
(\mathrm{n}=28) \text { or endarterectomy }(\mathrm{n}=35) \text { between January } 2007 \text { and December } 2012\end{array}$ \\
\hline Participants & $\begin{array}{l}\text { Inclusion criteria: bilateral or unilateral asymptomatic carotid stenosis } \geq 70 \% \text { or symptomatic carotid } \\
\text { stenosis }>50 \%\end{array}$ \\
& $\begin{array}{l}\text { Exclusion criteria: intracranial haemorrhage within } 3 \text { months of enrolment, participants with "not ide- } \\
\text { ally managed" hypertension, haemorrhagic diathesis, complete occlusion of the carotid artery, loca- } \\
\text { tion of stenosis not accessible by either intervention, intracranial aneurysm, malignant tumours }\end{array}$
\end{tabular}

Interventions

Endovascular treatment: all participants were administered clopidogrel $75 \mathrm{mg}$ daily and aspirin $300 \mathrm{mg}$ daily, distal protection devices were used in all participants (no details about device provided), participants were administered clopidogrel $75 \mathrm{mg}$ daily and aspirin $300 \mathrm{mg}$ daily for 3 months, aspirin 100 $\mathrm{mg} /$ day and clopidogrel $75 \mathrm{mg} /$ day for 3 months thereafter.

Endarterectomy: general anaesthesia was administered in all participants, the use of shunts and patches was left at the discretion of the surgeon, postoperative aspirin $100 \mathrm{mg}$ daily was administered indefinitely.

\begin{tabular}{ll}
\hline Outcomes & Primary endpoint: adverse cardiac events, death, and stroke within 1 month after treatment, ipsilateral \\
stroke or death during 6 months of follow-up \\
Secondary endpoints: hyperperfusion syndrome, haemodynamic disorders, local haematoma, periph- \\
eral nerve injury, severe restenosis after 1 year
\end{tabular}

$\begin{array}{ll}\text { Follow-up } & 7 \text { days; and } 1,6 \text {, and } 12 \text { months after treatment by digital subtraction angiography, CTA, and ultra- } \\ \text { sound as well as clinically }\end{array}$

Funding Not specified


Beijing 2013 (Continued)

Trial registration number

No registration found

\section{Notes}

\section{Risk of bias}

\begin{tabular}{|c|c|c|}
\hline Bias & Authors' judgement & Support for judgement \\
\hline \multirow[t]{2}{*}{$\begin{array}{l}\text { Random sequence genera- } \\
\text { tion (selection bias) }\end{array}$} & Unclear risk & $\begin{array}{l}\text { Quote: "Total } 63 \text { patients who underwent CEA or CAS ... were randomly divid- } \\
\text { ed into an observation group and a control group." }\end{array}$ \\
\hline & & Comment: method of randomisation unknown. \\
\hline $\begin{array}{l}\text { Allocation concealment } \\
\text { (selection bias) }\end{array}$ & Unclear risk & Comment: method of randomisation unknown. \\
\hline $\begin{array}{l}\text { Blinding of participants } \\
\text { and personnel (perfor- } \\
\text { mance bias) } \\
\text { All outcomes }\end{array}$ & Low risk & $\begin{array}{l}\text { Comment: blinding not possible, but the outcome was unlikely to have been } \\
\text { influenced. }\end{array}$ \\
\hline $\begin{array}{l}\text { Blinding of outcome as- } \\
\text { sessment (detection bias) } \\
\text { All outcomes }\end{array}$ & Unclear risk & Comment: no information available \\
\hline $\begin{array}{l}\text { Incomplete outcome data } \\
\text { (attrition bias) }\end{array}$ & Unclear risk & Numbers of participants at risk during follow-up not provided. \\
\hline All outcomes & & Comment: bias possible \\
\hline $\begin{array}{l}\text { Selective reporting (re- } \\
\text { porting bias) }\end{array}$ & Unclear risk & $\begin{array}{l}\text { Comment: no reference to a study protocol was made but the generally ex- } \\
\text { pected major outcome events were reported. }\end{array}$ \\
\hline
\end{tabular}

Houston 2014

Single-centre randomised controlled trial (USA)
1:1 randomisation between CEA and endovascular therapy
No predefined sample size documented, 60 participants were enrolled in the trial of whom 29 were as-
signed to carotid stenting and 31 to endarterectomy

Participants $\begin{aligned} & \text { Inclusion criteria: aged } \geq 40 \text { years, }>80 \% \text { asymptomatic carotid stenosis confirmed by } \geq 2 \text { diagnostic } \\ & \text { modalities (including duplex ultrasound, magnetic resonance angiography, CTA, or digital subtraction } \\ & \text { angiography) } \\ & \text { Exclusion criteria: previous neck radiotherapy or neck dissection, extensive calcification of the carotid } \\ & \text { artery, tracheostomy, carotid bifurcation above the second cervical vertebra, people with multiple co- } \\ & \text { morbidities who were considered unsuitable for endarterectomy, and people who scored }<24 \text { points } \\ & \text { on the Mini-Mental State Examination at baseline }\end{aligned}$

Interventions

Endarterectomy: performed under general anaesthesia with selective shunting, the use of patches was mandatory.

Carotid stenting: performed under local anaesthesia, stents had to be Food and Drug Administration approved. The use of embolic protection devices was mandatory. 
Houston 2014 (Continued)

Outcomes

Primary endpoint: any difference in cognitive tests of memory at 6 weeks between the 2 treatment groups

Secondary outcomes: difference in other domain-specific cognitive tests between treatment groups at 6 months (domains included processing speed, executive functioning, visual-spatial skills, motor skills, attention and mood).

\begin{tabular}{ll}
\hline Follow-up & 6 weeks and 6 months clinically and with neuropsychological testing \\
\hline Funding & Not specified \\
\hline Trial registration number & NCT02220595 \\
\hline
\end{tabular}

\section{Notes}

\section{Risk of bias}

\begin{tabular}{|c|c|c|}
\hline Bias & Authors' judgement & Support for judgement \\
\hline \multirow[t]{2}{*}{$\begin{array}{l}\text { Random sequence genera- } \\
\text { tion (selection bias) }\end{array}$} & Low risk & $\begin{array}{l}\text { Quote: "... a computer-generated code was used to randomly assign patients } \\
\text { to treatment ..." }\end{array}$ \\
\hline & & Comment: adequate \\
\hline \multirow[t]{2}{*}{$\begin{array}{l}\text { Allocation concealment } \\
\text { (selection bias) }\end{array}$} & Low risk & $\begin{array}{l}\text { Quote: "... a computer-generated code was used to randomly assign patients } \\
\text { to treatment ..." }\end{array}$ \\
\hline & & Comment: adequate \\
\hline $\begin{array}{l}\text { Blinding of participants } \\
\text { and personnel (perfor- } \\
\text { mance bias) } \\
\text { All outcomes }\end{array}$ & Low risk & $\begin{array}{l}\text { Comment: blinding not possible, but the outcome was unlikely to have been } \\
\text { influenced. }\end{array}$ \\
\hline $\begin{array}{l}\text { Blinding of outcome as- } \\
\text { sessment (detection bias) } \\
\text { All outcomes }\end{array}$ & Low risk & $\begin{array}{l}\text { Quote: "Tests were administered using standardized conditions and were } \\
\text { scored by individuals blinded to treatment allocation." }\end{array}$ \\
\hline \multirow[t]{2}{*}{$\begin{array}{l}\text { Incomplete outcome data } \\
\text { (attrition bias) } \\
\text { All outcomes }\end{array}$} & Low risk & $\begin{array}{l}3(5 \%) \text { participants were lost to follow-up and } 1 \text { participant in each treatment } \\
\text { group withdrew from the study after treatment. No information on clinical out- } \\
\text { come was available from excluded participants; however, the numbers were } \\
\text { small and the risk of bias was considered low. }\end{array}$ \\
\hline & & Comment: risk of attrition bias considered low. \\
\hline
\end{tabular}

\begin{tabular}{l}
$\begin{array}{l}\text { Selective reporting (re- Low risk Comment: the primary analysis prespecified in the protocol was reported. } \\
\text { porting bias) }\end{array}$ \\
\hline
\end{tabular}

Ostrava 2014

Methods Single-centre randomised controlled trial (Czech Republic)

Planned sample size of 146 participants, 150 participants were randomly assigned to either endovascular treatment $(n=77)$ or endarterectomy $(n=73)$ between October 2010 and June 2015.

All participants received the randomly allocated treatment. No cross-overs were reported. 
Ostrava 2014 (Continued)

Participants

Inclusion criteria: asymptomatic or symptomatic carotid stenosis $>70 \%$ documented by ultrasound and confirmed by CTA, aged $40-80$ years, functionally independent people defined as modified Rankin score $0-2$ points

Exclusion criteria: contraindications for MRI, CTA, or digital subtraction angiography

Interventions

Endarterectomy: all procedures were performed under general anaesthesia, the use of shunt was left to the discretion of the surgeon, all participants were administered aspirin $100 \mathrm{mg}$ daily in the perioperative phase, a dose of 100 units per kg bodyweight unfractionated heparin was administered before flow arrest in the carotid artery, protamine was given 5 minutes after flow restoration in the ICA, clopidogrel $75 \mathrm{mg}$ daily was administered for 5 days after surgery.

Endovascular therapy: all procedures were performed via femoral access, all participants received long-term aspirin $100 \mathrm{mg}$ daily and were administered a loading dose of clopidogrel $525 \mathrm{mg}$. Cerebral protection devices (FilterWire EZ, Boston Scientific, USA) were used in all but 3 participants. The choice of stenting device was left at the discretion of the interventionist.

Outcomes

Primary endpoint: incidence of a new ischaemic lesion, defined as a hyperintense lesion on the postintervention DW-MRI that had not been present on the preintervention MRI, $>0.5 \mathrm{~cm}^{3}$ on brain DW-MRI performed 24 hours after treatment.

Secondary endpoints: changes in cognitive tests after 24 hours and 30 days, incidence of postinterventional stroke or TIA at 30 days, death, any stroke or MI within 30 days after the procedure.

\begin{tabular}{ll}
\hline Follow-up & DW-MRI 24 hours after treatment \\
& Clinically at 24 hours and 30 days after treatment
\end{tabular}

Funding Internal Grant Agency of the Ministry of Health of the Czech Republic

University Hospital Ostrava

Ministry of Education, Youth and Sports of the Czech Republic

\begin{tabular}{ll}
\hline Trial registration number $\quad$ NCT01591005 \\
\hline Notes
\end{tabular}

\section{Risk of bias}

\begin{tabular}{|c|c|c|}
\hline Bias & Authors' judgement & Support for judgement \\
\hline $\begin{array}{l}\text { Random sequence genera- } \\
\text { tion (selection bias) }\end{array}$ & Low risk & $\begin{array}{l}\text { Quote: "Randomization was computer generated; patients were assigned } \\
\text { equally to CEA or CAS." } \\
\text { Comment: adequate }\end{array}$ \\
\hline $\begin{array}{l}\text { Blinding of participants } \\
\text { and personnel (perfor- } \\
\text { mance bias) } \\
\text { All outcomes }\end{array}$ & Low risk & $\begin{array}{l}\text { Comment: blinding not possible, but the outcome was unlikely to have been } \\
\text { influenced. }\end{array}$ \\
\hline $\begin{array}{l}\text { Blinding of outcome as- } \\
\text { sessment (detection bias) } \\
\text { All outcomes }\end{array}$ & Low risk & $\begin{array}{l}\text { Quote: "Physical and neurological examination was carried out by a blinded } \\
\text { neurologist before, and } 24 \mathrm{~h} \text { [ } 24 \text { hours] and } 30 \text { days after intervention." }\end{array}$ \\
\hline
\end{tabular}


Ostrava 2014 (Continued)

Comment: influence on outcome judged unlikely.

$\begin{array}{ll}\begin{array}{l}\text { Incomplete outcome data } \\ \text { (attrition bias) }\end{array} & \text { Low risk } \\ \text { All outcomes } & \begin{array}{l}\text { No participants were excluded from analysis. All participants received the ran- } \\ \text { domly allocated treatment and outcome events for all participants were pro- } \\ \text { vided. }\end{array}\end{array}$

Comment: risk of attrition bias considered low.

\begin{tabular}{ll}
\hline $\begin{array}{l}\text { Selective reporting (re- } \\
\text { porting bias) }\end{array}$ & Low risk \\
pected major outcome events were reported.
\end{tabular}

\begin{tabular}{|c|c|}
\hline \multirow[t]{2}{*}{ Methods } & Multicentre randomised trial (USA) \\
\hline & $\begin{array}{l}\text { Planned sample size of } 1658 \text { participants at participating centres, the study was terminated early due } \\
\text { to slow enrolment, } 1453 \text { participants randomly assigned in a 3:1 ratio to carotid stenting }(n=1089) \text { or } \\
\text { endarterectomy }(n=364) \text { between April } 2005 \text { and March } 2013 \text {. }\end{array}$ \\
\hline \multirow[t]{3}{*}{ Participants } & $\begin{array}{l}\text { Inclusion criteria: asymptomatic carotid stenosis, defined as having been free, in the ipsilateral hemi- } \\
\text { sphere, from stroke, TIA, and amaurosis fugax for } 180 \text { days before enrolment, of } 70-99 \% \text { documented } \\
\text { on either ultrasound or angiography, absence }>60 \% \text { contralateral carotid stenosis, aged } \leq 79 \text { years }\end{array}$ \\
\hline & $\begin{array}{l}\text { Exclusion criteria: intracranial haemorrhage or haemorrhagic stroke within } 1 \text { year prior to enrolment, } \\
\text { intolerance or allergic reaction to any study medications (including aspirin, clopidogrel, ticlopidine, he- } \\
\text { parin, or bivalirudin), MI within the previous } 30 \text { days, coexisting condition that limited their anticipated } \\
\text { survival to }<3 \text { years, considered a high-risk surgical candidate }\end{array}$ \\
\hline & Mean age 68 years, $59 \%$ men \\
\hline
\end{tabular}

Interventions

Carotid stenting: insertion of a closed-cell, nitinol stents with tapering diameter (Xact, Abbott Vascular) in conjunction with distal embolic protection (Emboshield, Emboshield Pro or Emboshield NAV6, Abbott Vascular)

Endarterectomy: type of anaesthesia, the use of patches or shunts and intraoperative monitoring were left to the discretion of the surgeon.

The interventionists performing the stenting procedures were qualified by Abbott Vascular (sponsor). Lead-in phase of 200 participants to allow investigators to gain experience with the study devices.

Surgeons had to be approved by the sponsor (Abbott Vascular, USA).

All participants received aspirin $325 \mathrm{mg}$ daily starting 3 days before the procedure and indefinitely after the procedure. Participants who underwent stenting received clopidogrel for 3 days before the procedure and for 30 days thereafter.

Primary method of comparison: intention-to-treat
Primary endpoint: composite of death, stroke (ipsilateral or contralateral, major or minor), or MI during
30 days after the procedure or ipsilateral stroke during the 365 days after the procedure
Secondary endpoints: composite of cranial-nerve and peripheral-nerve injury, vascular injury, non-
cerebral bleeding, wound complications assessed at 30 days after treatment, freedom from clinical-
ly driven target-lesion revascularisation for 5 years, freedom from death for 5 years, freedom from all
stroke for 5 years


ACT-1 2016 (Continued)

Trial registration number

NCT00106938

\section{Notes}

\section{Risk of bias}

\begin{tabular}{|c|c|c|}
\hline Bias & Authors' judgement & Support for judgement \\
\hline $\begin{array}{l}\text { Random sequence genera- } \\
\text { tion (selection bias) }\end{array}$ & Low risk & $\begin{array}{l}\text { Quote: "Randomization was performed with the use of a Web-based system." } \\
\text { Comment: adequate }\end{array}$ \\
\hline $\begin{array}{l}\text { Allocation concealment } \\
\text { (selection bias) }\end{array}$ & Low risk & $\begin{array}{l}\text { Quote: "Randomization was performed with the use of a Web-based system." } \\
\text { Comment: adequate }\end{array}$ \\
\hline $\begin{array}{l}\text { Blinding of participants } \\
\text { and personnel (perfor- } \\
\text { mance bias) } \\
\text { All outcomes }\end{array}$ & Low risk & $\begin{array}{l}\text { Comment: blinding not possible, but the outcome was unlikely to have been } \\
\text { influenced. }\end{array}$ \\
\hline $\begin{array}{l}\text { Blinding of outcome as- } \\
\text { sessment (detection bias) } \\
\text { All outcomes }\end{array}$ & Low risk & $\begin{array}{l}\text { Quote: "An independent clinical events committee from the Harvard Clinical } \\
\text { Research Institute adjudicated all primary end-point events, and an indepen- } \\
\text { dent data and safety monitoring board from the Harvard Clinical Research In- } \\
\text { stitute reviewed the accumulating data." } \\
\text { Comment: influence on outcome judged unlikely. }\end{array}$ \\
\hline $\begin{array}{l}\text { Incomplete outcome data } \\
\text { (attrition bias) } \\
\text { All outcomes }\end{array}$ & Low risk & $\begin{array}{l}\text { Numbers of participants lost to follow-up or participants who withdrew con- } \\
\text { sent up to } 1 \text { year after enrolment were provided ( } 54 \text { participants withdrew } \\
\text { consent, } 7 \text { were lost to follow-up). }\end{array}$ \\
\hline $\begin{array}{l}\text { Selective reporting (re- } \\
\text { porting bias) }\end{array}$ & Low risk & Comment: the primary analysis prespecified in the protocol was reported. \\
\hline
\end{tabular}

SPACE-2 2016

Methods Multicentre 3-armed trial (BMT vs BMT plus endarterectomy vs BMT plus endovascular treatment) with a 2:2:1 randomisation (Germany, Austria, and Switzerland)

Hierarchical study design; first aim was to establish the superiority of both interventions (endovascular therapy and endarterectomy) compared to BMT alone; second aim, a non-inferiority comparison of endovascular treatment vs endarterectomy was planned

In July 2013 the 3-armed study design was amended to become 2 parallel randomised controlled trials; the first, a randomised comparison of BMT vs endarterectomy and the second, BMT vs endovascular treatment.

Planned sample size 3640 participants, enrolment was stopped early after inclusion of 513 participants between March 2009 and October 2014 due to slow recruitment.

Participants Inclusion criteria: $\geq 70 \%$ (ECST) carotid stenosis documented on ultrasound, no stroke or stroke-like symptoms attributable to the stenosis within 180 days of enrolment, aged 50-85 years

Exclusion criteria: non-atherosclerotic stenosis (dissection, fibromuscular dysplasia, previous radiotherapy of the neck), additional higher-grade intracranial or intrathoracic stenosis (tandem stenosis), 
SPACE-2 2016 (Continued)

intracranial bleeding within the last 90 days, pre-existing disability resulting in a modified Rankin scale $>1$, life expectancy $<5$ years

Interventions

BMT: consisted of risk factor modification tailored to the individual risk factor profile of each participant including lipid-lowering medication, antiplatelet medication, and antihypertensive medication.

Endovascular treatment: participants had to be administered aspirin 100-325 mg daily and clopidogrel $75 \mathrm{mg}$ daily for $\geq 3$ days prior to the intervention. Transfemoral or transbrachial approach was possible. Stent devices had to be CE-certified. The use of protection devices was left to the discretion of the interventionist. After the intervention, aspirin had to be continued indefinitely and clopidogrel for 4-6 weeks. All interventionists had to have performed $\geq 10$ procedures within the SPACE- 1 trial or $\geq 40$ procedures in the last 24 months.

Endarterectomy: type of anaesthesia, endarterectomy technique (eversion or standard), use of patches or shunts was left to the discretion of the surgeon. Participants had to be administered aspirin 50-325 $\mathrm{mg}$ daily or clopidogrel $75 \mathrm{mg}$ daily for $\geq 3$ days prior to the operation. Surgeons had to have an individual experience of $>200$ endarterectomies in total and had to have performed $\geq 40$ endarterectomies in the last 2 years.

Outcomes

30-day safety endpoint: composite of any stroke or death of any cause within 30 days after treatment (only assessed in participants who were randomised to endarterectomy or endovascular treatment)

Primary efficacy endpoint: cumulative rate of any stroke or death from any cause within 30 days or ipsilateral ischaemic stroke within 5 years of follow-up

Secondary endpoints: MI within 30 days, technical failure of intervention, rate of restenosis $(\geq 70 \%$ ECST) up to 5 years after randomisation

Follow-up All participants were followed clinically and with ultrasound after 30 days, 6 and 12 months, and annually thereafter.

Participants who had undergone either endarterectomy or endovascular treatment were also followed clinically and with ultrasound 1 day after treatment and ECG was performed.

Funding

BMBF German Federal Ministry of Education and Research

DFG Deutsche Forschungsgemeinschaft (German Research Foundation)

Trial registration number ISRCTN78592017

Notes

\section{Risk of bias}

\begin{tabular}{lll}
\hline Bias & Authors' judgement & Support for judgement \\
\hline $\begin{array}{l}\text { Random sequence genera- } \\
\text { tion (selection bias) }\end{array}$ & Low risk & $\begin{array}{l}\text { Quote: "Randomisation will be accomplished with an Internet-based system } \\
\text { provided by the data centre in Munich. Randomisation will be stratified by } \\
\text { an age limit of } 75 \text { years, providing the same number of instances per group in } \\
\text { both age cohorts." }\end{array}$ \\
\hline $\begin{array}{l}\text { Allocation concealment } \\
\text { (selection bias) }\end{array}$ & Low risk & $\begin{array}{l}\text { Comment: adequate } \\
\text { provided by the data centre in Munich. Randomisation will be stratified by } \\
\text { an age limit of } 75 \text { years, providing the same number of instances per group in } \\
\text { both age cohorts." }\end{array}$ \\
& \begin{tabular}{l} 
Comment: adequate \\
\hline
\end{tabular} &
\end{tabular}


SPACE-2 2016 (Continued)

Blinding of participants Low risk Comment: blinding not possible, but the outcome was unlikely to have been and personnel (perforinfluenced.

mance bias)

All outcomes

\section{Blinding of outcome as- Low risk} sessment (detection bias)

All outcomes
Quote: "However, to minimise potential bias due to the open design, a physician, not directly involved in the CAS or the CEA procedure, is responsible for the follow-up examinations and end-point evaluation."

Comment: influence on outcome judged unlikely
Incomplete outcome data Unclear risk

(attrition bias)

All outcomes
Only 513 participants were included in the trial. There are currently no data available on excluded participants.

Selective reporting (re- Low risk Comment: the primary analysis prespecified in the protocol was reported.
porting bias)

\title{
Carmel Medical Center 2017
}

\begin{tabular}{|c|c|}
\hline \multirow[t]{3}{*}{ Methods } & Single-centre randomised controlled trial (USA) \\
\hline & 1:1 randomisation between CEA and carotid stenting \\
\hline & $\begin{array}{l}\text { Planned sample size: } 500 \text { participants, } 136 \text { participants were enrolled in the trial of whom } 68 \text { were as- } \\
\text { signed to endovascular treatment and } 68 \text { to endarterectomy }\end{array}$ \\
\hline
\end{tabular}

\begin{abstract}
Participants Inclusion criteria: severe carotid stenosis (> 70\%), no ipsilateral carotid territory symptoms for $\geq 6$ months, no previous ipsilateral carotid procedure, both endarterectomy and carotid stenting were thought possible, life expectancy $\geq 5$ years

Exclusion criteria: substantial atherosclerosis of the aortic arch or origin of the carotid artery (or both), significantly tortuous common or ICA, highly calcified stenosis, suspected thrombus or ulcer
\end{abstract}

Endarterectomy: performed under regional anaesthesia, and the use of shunts, patches, and standard
or eversion endarterectomy technique was at the discretion of the surgeon.
CAS: performed through a transfemoral access. The protocol specified the use of distal protection sys-
tems (either Angioguard, Cordis or SpiderFX, Covidien) and Precise-rapid exchange stent. All partici-
pants in the endovascular treatment arm received clopidogrel $75 \mathrm{mg}$ the day before treatment and for
45 days after treatment.

Primary outcome: periprocedural complications defined as any events occurring within the first month
after treatment including MI (defined by relevant clinical symptoms and elevated cardiac enzymes),
TIA, stroke, or death; and death, ipsilateral stroke, or TIA, severe $(\geq 70 \%)$ restenosis within 5 years after
treatment

\begin{tabular}{ll}
\hline Follow-up & Clinically and with duplex ultrasound at 1, 3, and 6 months after treatment, and annually thereafter for \\
up to 5 years
\end{tabular}

\begin{tabular}{ll}
\hline Funding & Not specified \\
\hline Trial registration number & NCT00772278 \\
\hline
\end{tabular}

\section{Notes}

\section{Risk of bias}

Carotid artery stenting versus endarterectomy for treatment of carotid artery stenosis (Review) 
Carmel Medical Center 2017 (Continued)

\begin{tabular}{|c|c|c|}
\hline Bias & Authors' judgement & Support for judgement \\
\hline $\begin{array}{l}\text { Random sequence genera- } \\
\text { tion (selection bias) }\end{array}$ & Unclear risk & $\begin{array}{l}\text { No information on method of randomisation specified. } \\
\text { Comment: unclear }\end{array}$ \\
\hline $\begin{array}{l}\text { Allocation concealment } \\
\text { (selection bias) }\end{array}$ & Unclear risk & $\begin{array}{l}\text { Quote: see above (no information on method of randomisation specified) } \\
\text { Comment: unclear }\end{array}$ \\
\hline $\begin{array}{l}\text { Blinding of participants } \\
\text { and personnel (perfor- } \\
\text { mance bias) } \\
\text { All outcomes }\end{array}$ & Low risk & $\begin{array}{l}\text { Comment: blinding not possible, but the outcome was unlikely to have been } \\
\text { influenced. }\end{array}$ \\
\hline $\begin{array}{l}\text { Blinding of outcome as- } \\
\text { sessment (detection bias) } \\
\text { All outcomes }\end{array}$ & Unclear risk & Comment: no information available. \\
\hline $\begin{array}{l}\text { Incomplete outcome data } \\
\text { (attrition bias) } \\
\text { All outcomes }\end{array}$ & Low risk & $\begin{array}{l}3 \text { participants }(2.2 \%) \text { were lost to follow-up and } 1 \text { participant crossed over } \\
\text { from carotid stenting to endarterectomy. No information on clinical outcome } \\
\text { was available from excluded participants; however, the numbers were small } \\
\text { and the risk of bias was considered low. } \\
\text { Comment: risk of attrition bias considered low. }\end{array}$ \\
\hline $\begin{array}{l}\text { Selective reporting (re- } \\
\text { porting bias) }\end{array}$ & Low risk & $\begin{array}{l}\text { Comment: no reference to a study protocol was made but the generally ex- } \\
\text { pected major outcome events were reported. }\end{array}$ \\
\hline
\end{tabular}

bd: twice daily; BMT: best medical treatment; CAS: carotid artery stenting; CE: certification mark; CEA: carotid endarterectomy; CT: computed tomography; CTA: computed tomography angiography; DW-MRI: diffusion-weighted magnetic resonance imaging; ECG: electrocardiography; ECST: European Carotid Surgery Trial; EEG: electroencephalography; ICA: internal carotid artery; iv: intravenous; MI: myocardial infarction; MRI: magnetic resonance imaging; n: number; NASCET: North American Symptomatic Carotid Endarterectomy Trial; NIHSS: National Institutes of Health Stroke Scale; TIA: transient ischaemic attack.

Characteristics of ongoing studies [ordered by study ID]

\section{ACST-2}

\begin{tabular}{|c|c|}
\hline Trial name or title & Asymptomatic Carotid Surgery Trial-2 (ACST-2) \\
\hline Methods & $\begin{array}{l}\text { Multicentre trial, 1:1 randomisation between endovascular treatment or endarterectomy } \\
\text { Planned sample size: } 3600 \text { participants }\end{array}$ \\
\hline Participants & $\begin{array}{l}\text { Inclusion criteria: aged } \geq 18 \text { years, carotid artery stenosis considered to require treatment, without } \\
\text { ipsilateral carotid territory symptoms "for some months;" patients fit and willing to be followed up } \\
\text { for } \geq 5 \text { years } \\
\text { Exclusion criteria: small likelihood of benefit from revascularisation, previous revascularisation in } \\
\text { the relevant artery, unsuitable for either procedure }\end{array}$ \\
\hline Interventions & $\begin{array}{l}\text { Carotid stenting: stent insertion without further specifications. The use of protection devices is op- } \\
\text { tional. } \\
\text { Endarterectomy: no specifications are made. } \\
\text { Interventionalists and surgeons must have performed } \geq 25 \text { procedures in the carotid artery to par- } \\
\text { ticipate in the trial. }\end{array}$ \\
\hline
\end{tabular}


ACST-2 (Continued)
Outcomes
Primary objectives:
- procedural risks of stroke, MI, and death, within the first month after procedure
- long-term prevention of stroke in subsequent years

\begin{tabular}{ll}
\hline Starting date & January 2008 \\
\hline Contact information & $\begin{array}{l}\text { Principal Investigator: Prof Alison Halliday, ACST Office, Nuffield Department of Surgical Sciences, } \\
\text { Level 6, John Radcliffe Hospital, Headley Way, Headington, Oxford OX3 9DU, UK (acst@nd- } \\
\text { s.ox.ac.uk) }\end{array}$
\end{tabular}

$\begin{array}{ll}\text { Follow-up } & \begin{array}{l}1 \text { month after treatment clinically, and then annually up to } 5 \text { years after treatment by question- } \\ \text { naires }\end{array}\end{array}$

\begin{tabular}{ll}
\hline Trial registration number & NCT00883402 \\
& ISRCTN21144362 \\
\hline Funding & National Institute of Health Research (NIHR) Health Technology Assessment programme, UK \\
& BUPA Foundation, UK \\
\hline Notes & \\
\hline
\end{tabular}

\begin{tabular}{|c|c|}
\hline Trial name or title & $\begin{array}{l}\text { Early invasive treatment (endarterectomy versus stenting) of moderate to severe carotid stenosis } \\
\text { in patients with transient ischaemic attack or minor stroke }\end{array}$ \\
\hline \multirow[t]{2}{*}{ Methods } & Multicentre trial, randomisation between endovascular treatment or endarterectomy \\
\hline & Planned sample size: 400 participants \\
\hline \multirow[t]{2}{*}{ Participants } & $\begin{array}{l}\text { Inclusion criteria: aged } \geq 18 \text { years, } 5-95 \% \text { carotid stenosis with ipsilateral TIA or stroke in the previ- } \\
\text { ous month }\end{array}$ \\
\hline & $\begin{array}{l}\text { Exclusion criteria: TIA or stroke in the previous } 48 \text { hours, endoluminal thrombus, tandem stenosis, } \\
\text { restenosis after previous endarterectomy, severe cardiopulmonary disease, life expectancy }<1 \text { year }\end{array}$ \\
\hline \multirow[t]{2}{*}{ Interventions } & Endovascular treatment: stent insertion \\
\hline & Endarterectomy: no specifications are provided. \\
\hline Outcomes & Primary outcome: cumulative incidence of TIA, stroke, or death at 2 years, or MI at 1 month \\
\hline Starting date & Unknown \\
\hline Contact information & $\begin{array}{l}\text { Principal Investigator: E Agostoni, Stroke Unit, Clinica Neurologica, Universita Milano-Bicocca, Mi- } \\
\text { lan, Italy } \\
\text { (e.agostoni@libero.it) }\end{array}$ \\
\hline Follow-up & 30 days; and 6,12 , and 24 months after treatment \\
\hline Trial registration number & No registration found \\
\hline Funding & No information available \\
\hline
\end{tabular}


Agostoni 2005 (Continued)

Notes Recruitment status unknown

No update found July 2018

\section{CREST-2}

$\begin{array}{ll}\text { Trial name or title } & \begin{array}{l}\text { Carotid REvascularization and medical management for asymptomatic carotid Stenosis Trial } \\ \text { (CREST-2) }\end{array}\end{array}$

\begin{tabular}{|c|c|}
\hline Methods & $\begin{array}{l}2 \text { multicentre randomised controlled trials comparing carotid revascularisation and intensive med- } \\
\text { ical management vs medical management alone. } 1 \text { trial randomises participants in a 1:1 ratio be- } \\
\text { tween CEA vs medical management alone, another trial randomises participants between CAS vs } \\
\text { medical management alone }\end{array}$ \\
\hline
\end{tabular}

Planned sample size: 2480 participants

Participants

Inclusion criteria: asymptomatic (within 180 days of randomisation) carotid stenosis $\geq 70 \%$ (defined by catheter angiography, CTA or MRA according to the NASCET method or ultrasound defined by flow velocity), $\geq 35$ years, modified Rankin Scale $\leq 1$

Exclusion criteria: people with prior stroke or TIA within 180 days of randomisation, known malignancy, any major surgery within the past 1 month, ipsilateral internal or common carotid occlusion, intracarotid floating thrombus

Interventions

Medical management: uniform, centrally directed, and consist of the following: lipid-lowering therapy, blood pressure control, antiplatelet therapy and risk factor modification (weight loss, smoking cessation, exercise, and diabetes management).

Carotid revascularisation: CEA or CAS.

Primary outcome measure: composite of stroke plus death within 30 days after randomisation and
ipsilateral stroke thereafter up to 4 years
Secondary outcome measures include: cognitive function; major stroke; effect modification based
on participant age, sex, severity of carotid stenosis, restenosis, risk factor level, and duration of
asymptomatic period

\begin{tabular}{ll}
\hline Starting date & December 2014 \\
\hline Contact information & Thomas G Brott, MD, Mayo Clinic, FL, USA \\
\hline Follow-up & 1 month, 6 months, and annually thereafter \\
\hline Trial registration number & NCT02089217 \\
\hline Funding & National Institute of Neurological Disorders and Stroke (NINDS), USA \\
\hline Notes & \\
\hline
\end{tabular}

\section{ECST-2}

\begin{tabular}{ll}
\hline Trial name or title & The 2nd European Carotid Surgery Trial \\
\hline Methods & $\begin{array}{l}\text { Multicentre randomised trial, 1:1 assignment between carotid revascularisation plus optimised } \\
\text { medical treatment vs optimised medical treatment alone }\end{array}$ \\
\hline
\end{tabular}


ECST-2 (Continued)

Planned sample size: 320 participants in pilot phase, 2000 participants in full trial

Participants Inclusion criteria: asymptomatic or low- to intermediate-risk symptomatic carotid stenosis based on a 5 -year carotid artery risk score $>50 \%,>18$ years

Exclusion criteria: modified Rankin score $>2$ for any reason, neurologically or medically unstable patients, coronary artery bypass grafting within 3 months prior to randomisation, planned major surgery within 6 weeks after enrolment, life expectancy $<2$ years, carotid stenosis caused by nonatherosclerotic disease such as dissection, fibromuscular disease or neck radiotherapy

Interventions

Carotid revascularisation: done with endarterectomy or stenting, which is specified by the centre before randomisation

Optimised medical treatment: includes all 3 of optimal antiplatelet, high-dose statin, and blood pressure-dependent antihypertensive therapy, as well as risk factor modification

\section{Outcomes}

Primary outcome measures: any stroke at any time, plus non-stroke death occurring within 30 days of revascularisation. For the safety MRI analysis: combined 2-year rate of cerebral infarction, cerebral haemorrhage, MI, or procedural death after randomisation

Secondary outcome measures: ipsilateral stroke, MI, TIA, or any hospitalisation for vascular disease during follow-up, disabling stroke during follow-up, ipsilateral restenosis or stenosis progression, cognitive impairment

\begin{tabular}{ll}
\hline Starting date & March 2012 \\
\hline Contact information & $\begin{array}{l}\text { Prof Martin M Brown, UCL Institute of Neurology, The National Hospital for Neurology and Neuro- } \\
\text { surgery, Queen Square, London, UK (martin.brown@ucl.ac.uk) }\end{array}$ \\
\hline Follow-up & $\begin{array}{l}\text { 48 hours and } 30 \text { days after treatment, } 6 \text { and } 12 \text { months after randomisation, and then annually for } \\
\geq 5 \text { years }\end{array}$ \\
\hline Trial registration number & ISRCTN97744893 \\
\hline Funding & National Institute of Health Research (NIHR) Health Technology Assessment programme, UK \\
\hline Notes & \\
\hline
\end{tabular}

\section{NCT02841098}

\begin{tabular}{ll}
\hline Trial name or title & $\begin{array}{l}\text { Endarterectomy combined with optimal medical therapy (OMT) vs OMT alone in patients with } \\
\text { asymptomatic severe atherosclerotic carotid artery stenosis at higher-than-average risk of ipsilat- } \\
\text { eral stroke (ACTRIS) }\end{array}$ \\
\hline Methods & $\begin{array}{l}\text { Multicentre trial, randomisation between optimal medical therapy alone or optimal medical thera- } \\
\text { py with endarterectomy }\end{array}$ \\
& Planned sample size: 700 participants \\
\hline Participants & $\begin{array}{l}\text { Inclusion criteria: } \geq 50 \text { years, no ipsilateral stroke or TIA within } 180 \text { days of randomisation, athero- } \\
\text { sclerotic carotid stenosis } 60-99 \% \text { according to NASCET criteria, } \geq 1 \text { of the following markers for ip- } \\
\text { silateral stroke risk } \\
\text { - TCD-detected microembolic signals } \\
\text { - Impairment of TCD-measured cerebral vasomotor reserve } \\
\text { - Rapid and severe stenosis progression }\end{array}$
\end{tabular}


NCT02841098 (Continued)

Exclusion criteria: previous revascularisation procedure in the artery to be randomised, pre-existing disability (modified Rankin score $>2$ ), low 5-year life expectancy
Endarterectomy: no specifications made

Optimal medical therapy: antiplatelet therapy, antihypertensive therapy, high-dose statin treatment, and risk factor modification

\section{Outcomes}

Primary outcome measure: any ipsilateral stroke within 6 years after randomisation or procedural (within 30 days after revascularisation) stroke or death

Secondary outcome measures include: $\mathrm{MI}$, any hospitalisation for vascular disease, complications of endarterectomy procedure (cranial nerve palsy, haematoma), depression, quality of life, disability, achievements of goals for each component of the optimal medical treatment

\begin{tabular}{ll}
\hline Starting date & September 2016 \\
\hline Contact information & Prof Jean-Louis Mas, Hôpital Sainte-Anne, Université Paris-Descartes, Paris, France \\
\hline Follow-up & 1 month, 6 months, then every 6 months thereafter \\
\hline Trial registration number & NCT02841098 \\
\hline Funding & No information available \\
\hline
\end{tabular}

Notes

CAS: carotid artery stenting; CEA: carotid endarterectomy; CT: computed tomography; CTA: computed tomography angiography; MI: myocardial infarction; MRA: magnetic resonance angiography; MRI: magnetic resonance imaging; TIA: transient ischaemic attack; TCD: transcranial Doppler.

\section{DATA AND ANALYSES}

\section{Comparison 1. Stenting or endarterectomy for symptomatic carotid stenosis}

\begin{tabular}{|c|c|c|c|c|}
\hline Outcome or subgroup title & $\begin{array}{l}\text { No. of } \\
\text { studies }\end{array}$ & $\begin{array}{l}\text { No. of } \\
\text { partici- } \\
\text { pants }\end{array}$ & Statistical method & Effect size \\
\hline $\begin{array}{l}1 \text { Primary safety outcome: death or any stroke be- } \\
\text { tween randomisation and } 30 \text { days after treatment }\end{array}$ & 10 & 5396 & $\begin{array}{l}\text { Odds Ratio (M-H, Random, 95\% } \\
\mathrm{Cl} \text { ) }\end{array}$ & $1.70[1.31,2.19]$ \\
\hline 1.1 Symptomatic carotid stenosis & 8 & 5184 & $\begin{array}{l}\text { Odds Ratio (M-H, Random, 95\% } \\
\mathrm{Cl} \text { ) }\end{array}$ & $1.74[1.30,2.33]$ \\
\hline $\begin{array}{l}\text { 1.2 Asymptomatic and symptomatic carotid } \\
\text { stenosis combined }\end{array}$ & 2 & 212 & $\begin{array}{l}\text { Odds Ratio (M-H, Random, 95\% } \\
\mathrm{Cl})\end{array}$ & $1.00[0.23,4.34]$ \\
\hline $\begin{array}{l}2 \text { Death or any stroke between randomisation and } \\
30 \text { days after treatment according to age }\end{array}$ & 6 & 4861 & $\begin{array}{l}\text { Odds Ratio (M-H, Random, 95\% } \\
\text { Cl) }\end{array}$ & $1.67[1.22,2.28]$ \\
\hline 2.1 Aged $<70$ years & 6 & 2453 & $\begin{array}{l}\text { Odds Ratio (M-H, Random, 95\% } \\
\mathrm{Cl} \text { ) }\end{array}$ & $1.11[0.74,1.64]$ \\
\hline
\end{tabular}




\begin{tabular}{|c|c|c|c|c|}
\hline Outcome or subgroup title & $\begin{array}{l}\text { No. of } \\
\text { studies }\end{array}$ & $\begin{array}{l}\text { No. of } \\
\text { partici- } \\
\text { pants }\end{array}$ & Statistical method & Effect size \\
\hline 2.2 Aged $\geq 70$ years & 6 & 2408 & $\begin{array}{l}\text { Odds Ratio (M-H, Random, 95\% } \\
\mathrm{Cl})\end{array}$ & $2.23[1.61,3.08]$ \\
\hline $\begin{array}{l}3 \text { Death or any stroke between randomisation and } \\
30 \text { days after treatment according to sex }\end{array}$ & 6 & 4861 & $\begin{array}{l}\text { Odds Ratio (M-H, Random, 95\% } \\
\mathrm{Cl} \text { ) }\end{array}$ & $1.69[1.21,2.35]$ \\
\hline 3.1 Men & 6 & 3395 & $\begin{array}{l}\text { Odds Ratio (M-H, Random, 95\% } \\
\mathrm{Cl} \text { ) }\end{array}$ & $1.82[1.10,3.02]$ \\
\hline 3.2 Women & 6 & 1466 & $\begin{array}{l}\text { Odds Ratio (M-H, Random, 95\% } \\
\mathrm{Cl})\end{array}$ & $1.52[0.96,2.41]$ \\
\hline $\begin{array}{l}4 \text { Death or any stroke between randomisation and } \\
30 \text { days after treatment according to minimum } \\
\text { pretrial experience in endovascular treatment of } \\
\text { carotid stenosis }\end{array}$ & 7 & 5080 & $\begin{array}{l}\text { Odds Ratio (M-H, Random, 95\% } \\
\mathrm{Cl} \text { ) }\end{array}$ & $1.76[1.32,2.36]$ \\
\hline $4.1 \leq 10$ procedures & 3 & 2456 & $\begin{array}{l}\text { Odds Ratio (M-H, Random, 95\% } \\
\text { Cl) }\end{array}$ & $2.21[1.56,3.13]$ \\
\hline $4.2>10$ procedures & 4 & 2624 & $\begin{array}{l}\text { Odds Ratio (M-H, Random, 95\% } \\
\mathrm{Cl} \text { ) }\end{array}$ & $1.37[0.98,1.94]$ \\
\hline $\begin{array}{l}5 \text { Death or major or disabling stroke between ran- } \\
\text { domisation and } 30 \text { days after treatment }\end{array}$ & 7 & 4983 & $\begin{array}{l}\text { Odds Ratio (M-H, Random, 95\% } \\
\mathrm{Cl})\end{array}$ & $1.36[0.97,1.91]$ \\
\hline $\begin{array}{l}6 \text { Death of any cause between randomisation and } \\
30 \text { days after treatment }\end{array}$ & 10 & 5264 & $\begin{array}{l}\text { Odds Ratio (M-H, Random, 95\% } \\
\text { Cl) }\end{array}$ & $1.35[0.69,2.61]$ \\
\hline $\begin{array}{l}7 \text { Any stroke between randomisation and } 30 \text { days } \\
\text { after treatment }\end{array}$ & 8 & 5113 & $\begin{array}{l}\text { Odds Ratio (M-H, Random, 95\% } \\
\mathrm{Cl} \text { ) }\end{array}$ & $1.78[1.38,2.29]$ \\
\hline $\begin{array}{l}8 \text { Fatal, major, or disabling stroke between ran- } \\
\text { domisation and } 30 \text { days after treatment }\end{array}$ & 7 & 4983 & $\begin{array}{l}\text { Odds Ratio (M-H, Random, 95\% } \\
\mathrm{Cl} \text { ) }\end{array}$ & $1.34[0.93,1.92]$ \\
\hline $\begin{array}{l}9 \text { Myocardial infarction between randomisation } \\
\text { and } 30 \text { days after treatment }\end{array}$ & 8 & 4994 & $\begin{array}{l}\text { Odds Ratio (M-H, Random, 95\% } \\
\mathrm{Cl})\end{array}$ & $0.47[0.24,0.94]$ \\
\hline $\begin{array}{l}10 \text { Death or any stroke or myocardial infarction } \\
\text { between randomisation and } 30 \text { days after treat- } \\
\text { ment }\end{array}$ & 6 & 4861 & $\begin{array}{l}\text { Odds Ratio (M-H, Random, 95\% } \\
\mathrm{Cl} \text { ) }\end{array}$ & $1.43[1.14,1.80]$ \\
\hline $\begin{array}{l}11 \text { Primary combined safety and efficacy out- } \\
\text { come: death or any stroke between randomisa- } \\
\text { tion and } 30 \text { days after treatment or ipsilateral } \\
\text { stroke until the end of follow-up }\end{array}$ & 8 & 5080 & $\begin{array}{l}\text { Odds Ratio (M-H, Random, 95\% } \\
\mathrm{Cl} \text { ) }\end{array}$ & $1.51[1.24,1.85]$ \\
\hline $\begin{array}{l}12 \text { Death or any stroke between randomisation } \\
\text { and end of follow-up }\end{array}$ & 9 & 5292 & $\begin{array}{l}\text { Odds Ratio (M-H, Random, 95\% } \\
\mathrm{Cl} \text { ) }\end{array}$ & $1.23[1.03,1.46]$ \\
\hline $12.16-24$ months' follow-up & 4 & 1627 & $\begin{array}{l}\text { Odds Ratio (M-H, Random, 95\% } \\
\mathrm{Cl})\end{array}$ & $1.33[0.75,2.37]$ \\
\hline
\end{tabular}




\begin{tabular}{|c|c|c|c|c|}
\hline Outcome or subgroup title & $\begin{array}{l}\text { No. of } \\
\text { studies }\end{array}$ & $\begin{array}{l}\text { No. of } \\
\text { partici- } \\
\text { pants }\end{array}$ & Statistical method & Effect size \\
\hline $12.2 \geq 4$ years' follow-up & 5 & 3665 & $\begin{array}{l}\text { Odds Ratio (M-H, Random, 95\% } \\
\mathrm{Cl} \text { ) }\end{array}$ & $1.24[1.02,1.51]$ \\
\hline $\begin{array}{l}13 \text { Any stroke during follow-up (periprocedural } \\
\text { events and people dying in the periprocedural pe- } \\
\text { riod excluded) }\end{array}$ & 6 & 4837 & $\begin{array}{l}\text { Odds Ratio (M-H, Random, 95\% } \\
\mathrm{Cl} \text { ) }\end{array}$ & $1.15[0.82,1.62]$ \\
\hline $13.118-24$ months' follow-up & 2 & 1204 & $\begin{array}{l}\text { Odds Ratio (M-H, Random, 95\% } \\
\mathrm{Cl} \text { ) }\end{array}$ & $0.97[0.52,1.82]$ \\
\hline $13.2 \geq 4$ years' follow-up & 4 & 3633 & $\begin{array}{l}\text { Odds Ratio (M-H, Random, 95\% } \\
\mathrm{Cl} \text { ) }\end{array}$ & $1.21[0.79,1.86]$ \\
\hline $\begin{array}{l}14 \text { Ipsilateral stroke during follow-up (periproce- } \\
\text { dural events and people dying in the periproce- } \\
\text { dural period excluded) }\end{array}$ & 6 & 4837 & $\begin{array}{l}\text { Odds Ratio (M-H, Random, 95\% } \\
\mathrm{Cl} \text { ) }\end{array}$ & $1.05[0.75,1.47]$ \\
\hline $14.118-24$ months' follow-up & 2 & 1204 & $\begin{array}{l}\text { Odds Ratio (M-H, Random, 95\% } \\
\mathrm{Cl} \text { ) }\end{array}$ & $1.07[0.45,2.54]$ \\
\hline $14.2 \geq 4$ years' follow-up & 4 & 3633 & $\begin{array}{l}\text { Odds Ratio (M-H, Random, 95\% } \\
\mathrm{Cl} \text { ) }\end{array}$ & $1.05[0.73,1.50]$ \\
\hline $\begin{array}{l}15 \text { Death or any stroke or myocardial infarction } \\
\text { between randomisation and } 30 \text { days after treat- } \\
\text { ment or ipsilateral stroke until end of follow-up }\end{array}$ & 7 & 4907 & $\begin{array}{l}\text { Odds Ratio (M-H, Random, 95\% } \\
\mathrm{Cl} \text { ) }\end{array}$ & $1.39[1.15,1.69]$ \\
\hline
\end{tabular}

Analysis 1.1. Comparison 1 Stenting or endarterectomy for symptomatic carotid stenosis, Outcome 1 Primary safety outcome: death or any stroke between randomisation and $\mathbf{3 0}$ days after treatment.

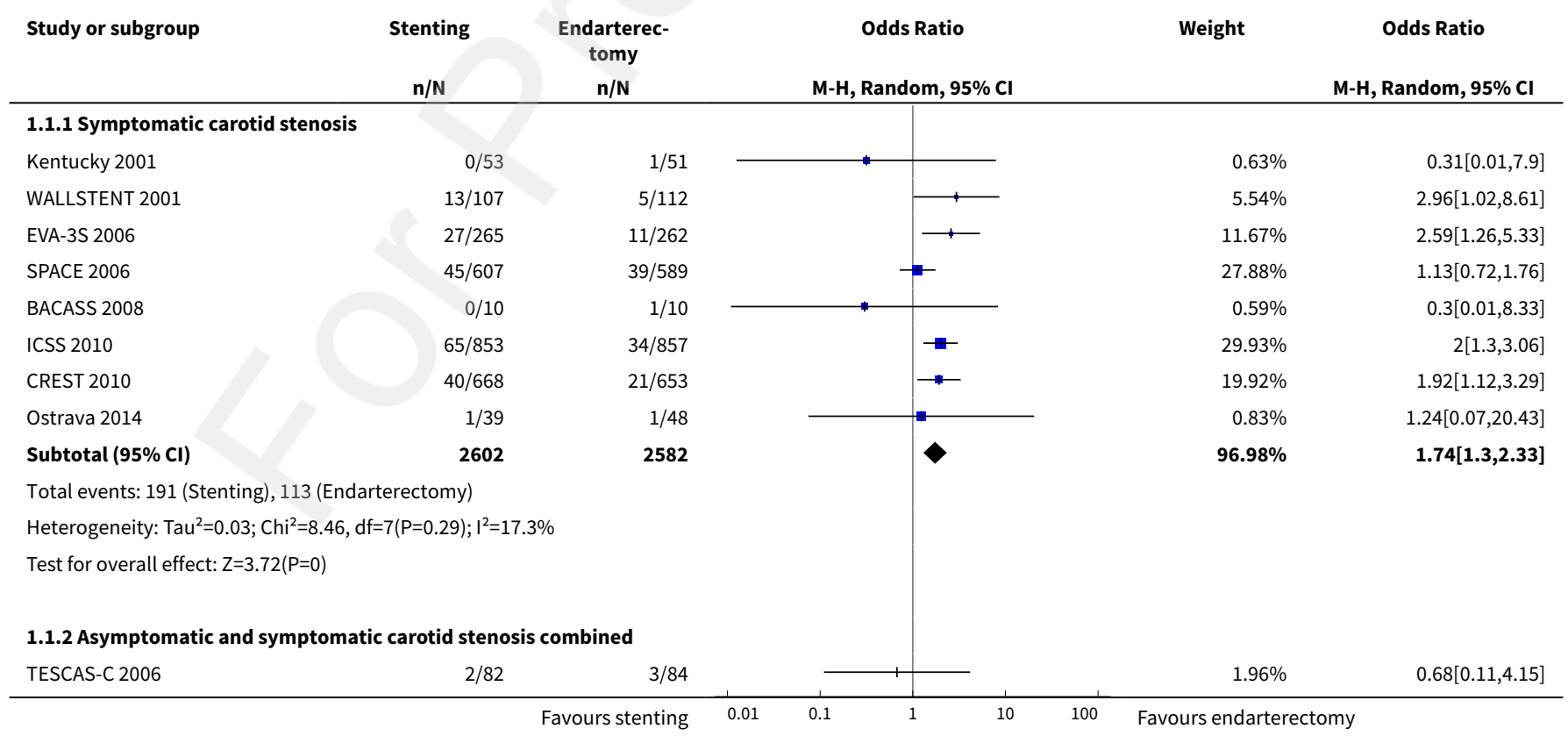




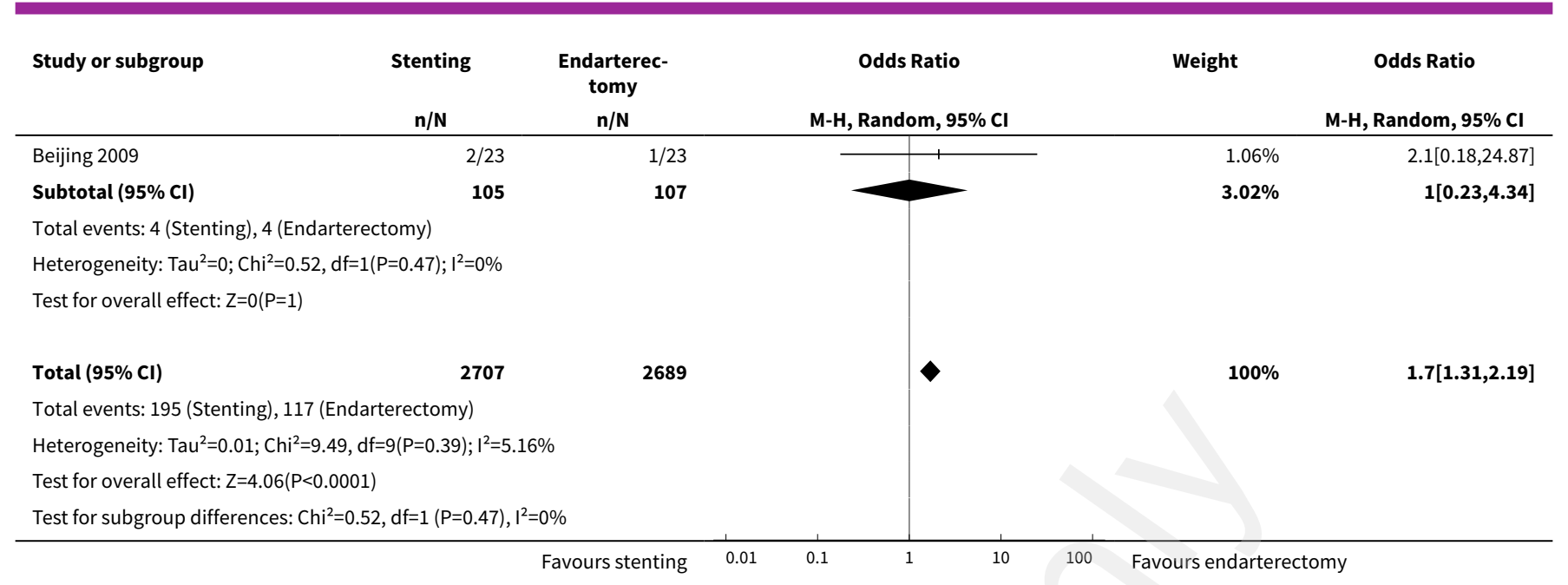

\section{Analysis 1.2. Comparison 1 Stenting or endarterectomy for symptomatic carotid stenosis, Outcome 2 Death or any stroke between randomisation and 30 days after treatment according to age.}

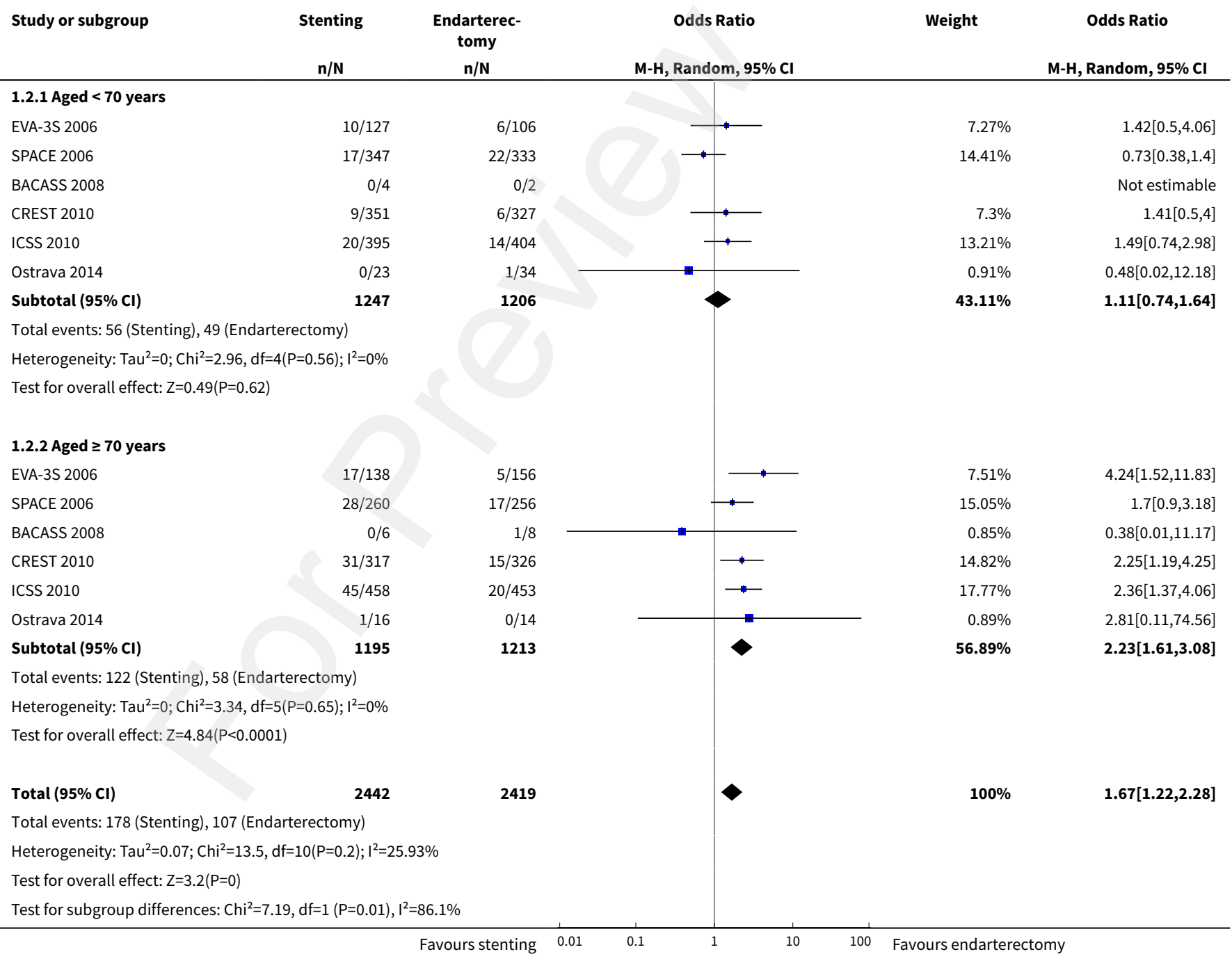


Analysis 1.3. Comparison 1 Stenting or endarterectomy for symptomatic carotid stenosis, Outcome 3 Death or any stroke between randomisation and $\mathbf{3 0}$ days after treatment according to sex.

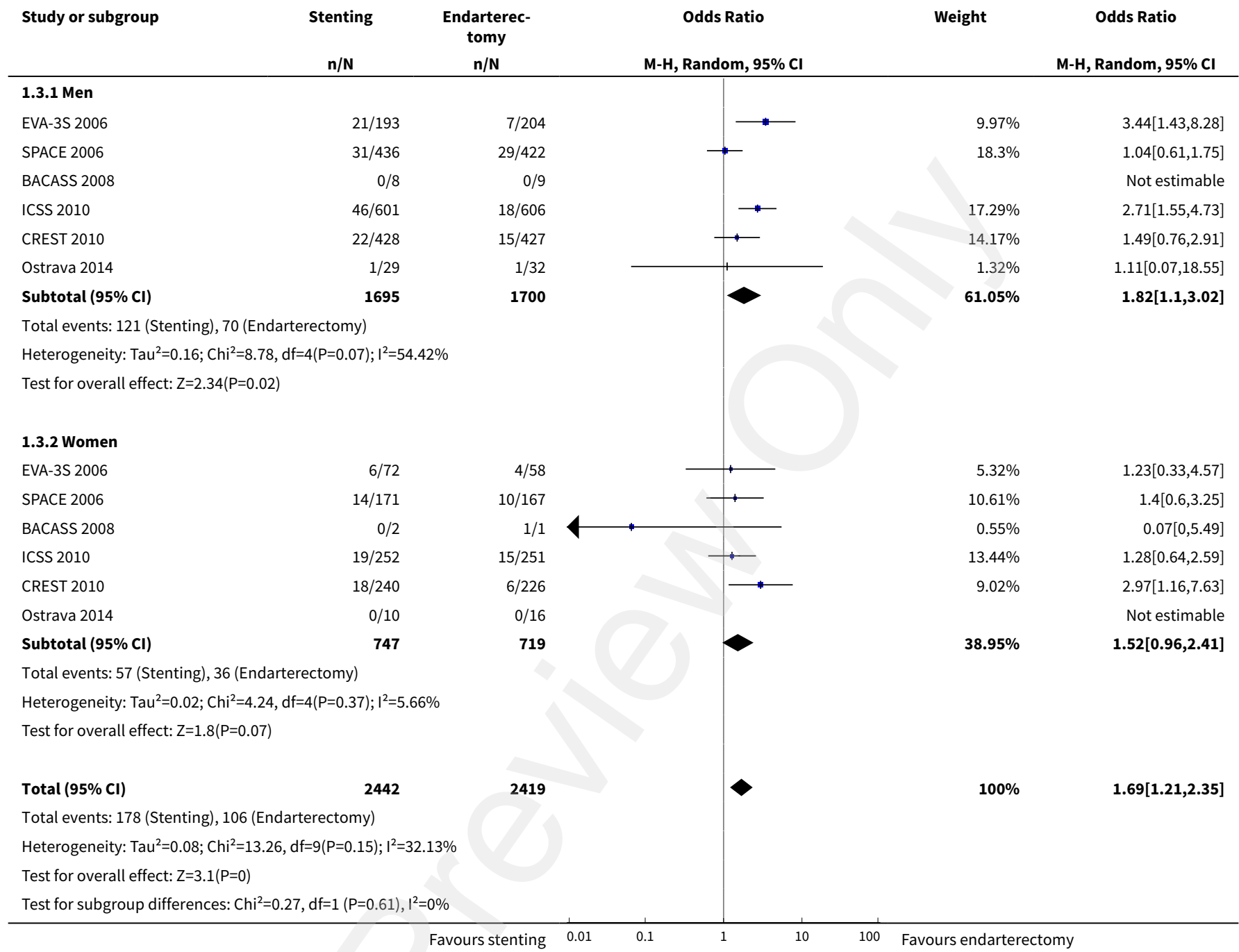

Analysis 1.4. Comparison 1 Stenting or endarterectomy for symptomatic carotid stenosis,

Outcome 4 Death or any stroke between randomisation and 30 days after treatment according to minimum pretrial experience in endovascular treatment of carotid stenosis.

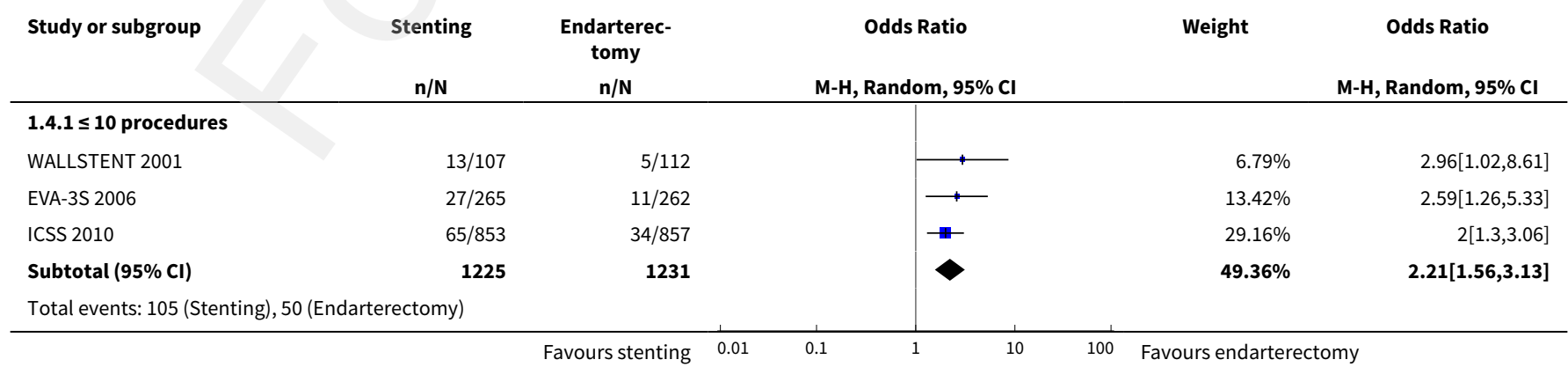




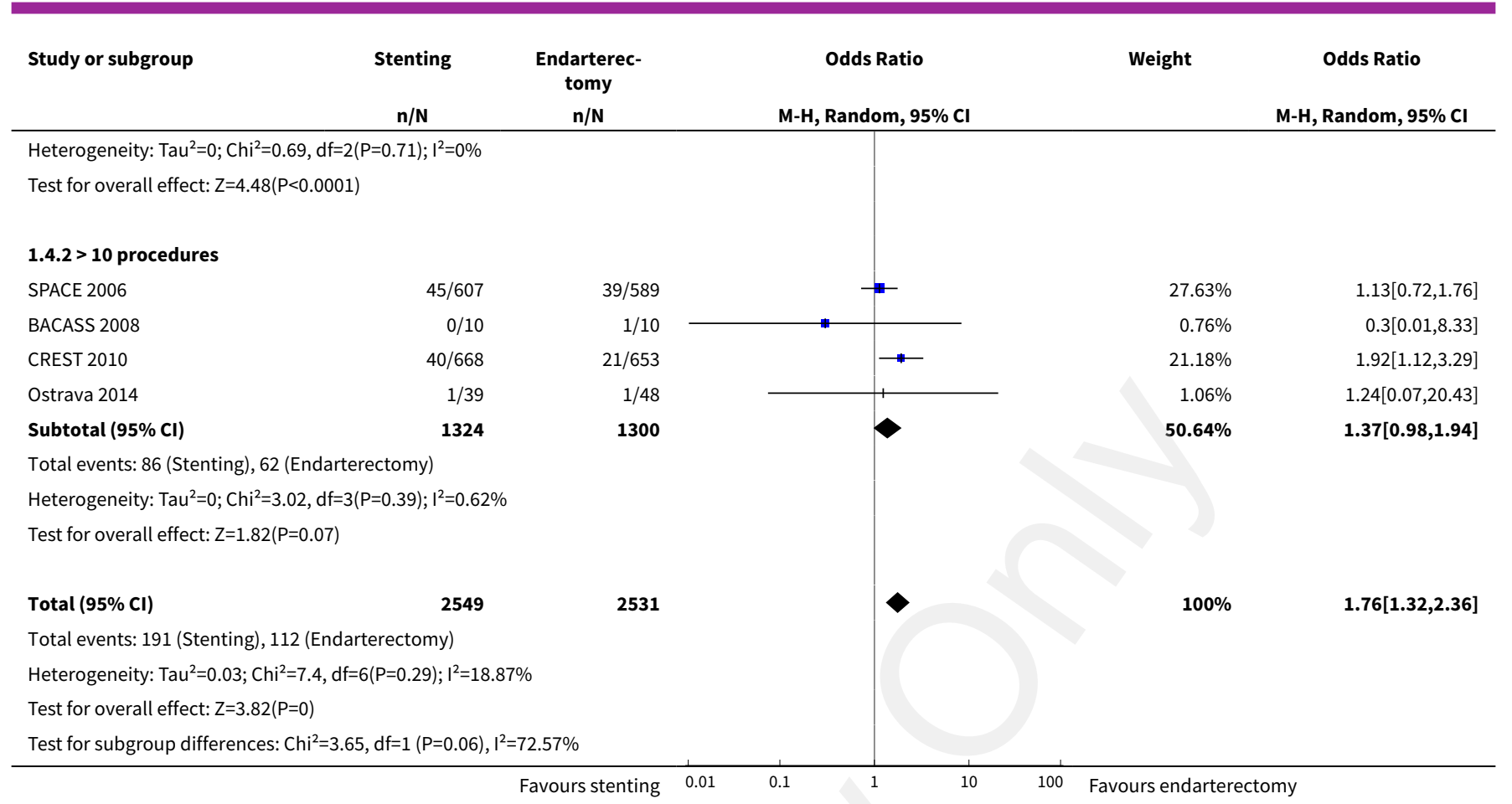

Analysis 1.5. Comparison 1 Stenting or endarterectomy for symptomatic carotid stenosis, Outcome 5 Death or major or disabling stroke between randomisation and $\mathbf{3 0}$ days after treatment.

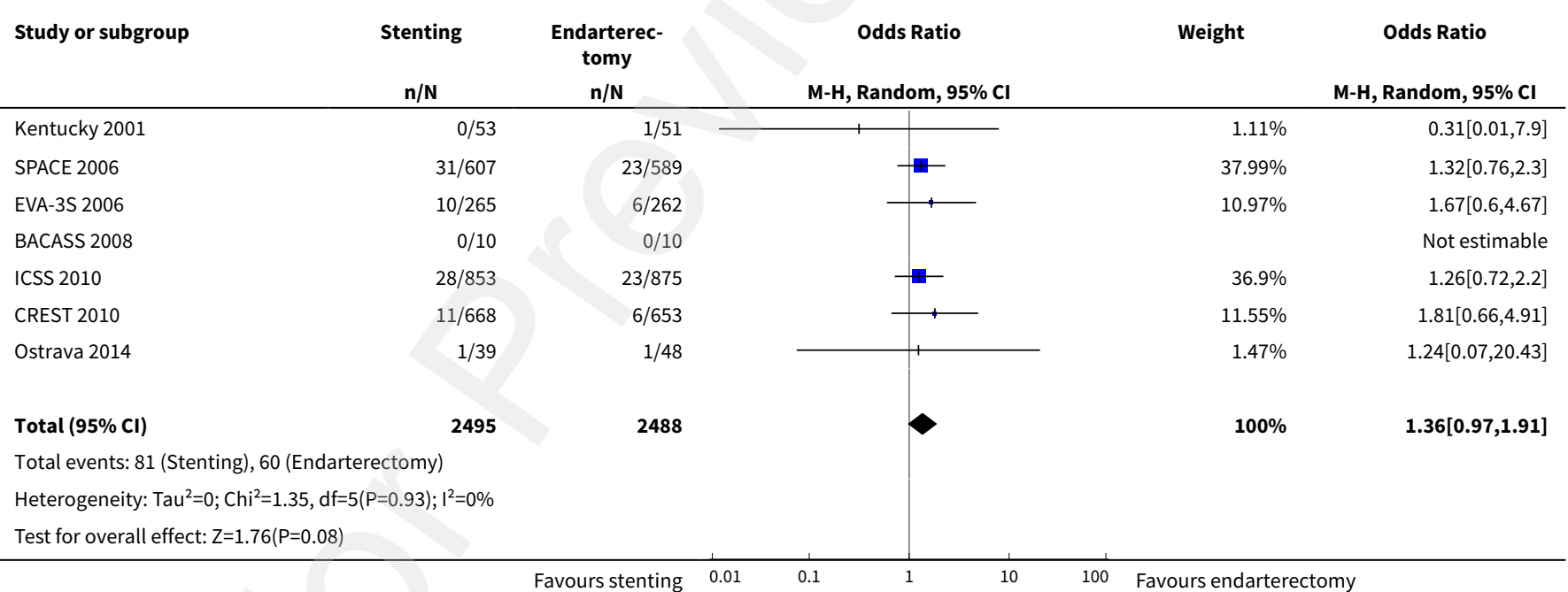


Analysis 1.6. Comparison 1 Stenting or endarterectomy for symptomatic carotid stenosis, Outcome 6 Death of any cause between randomisation and 30 days after treatment.

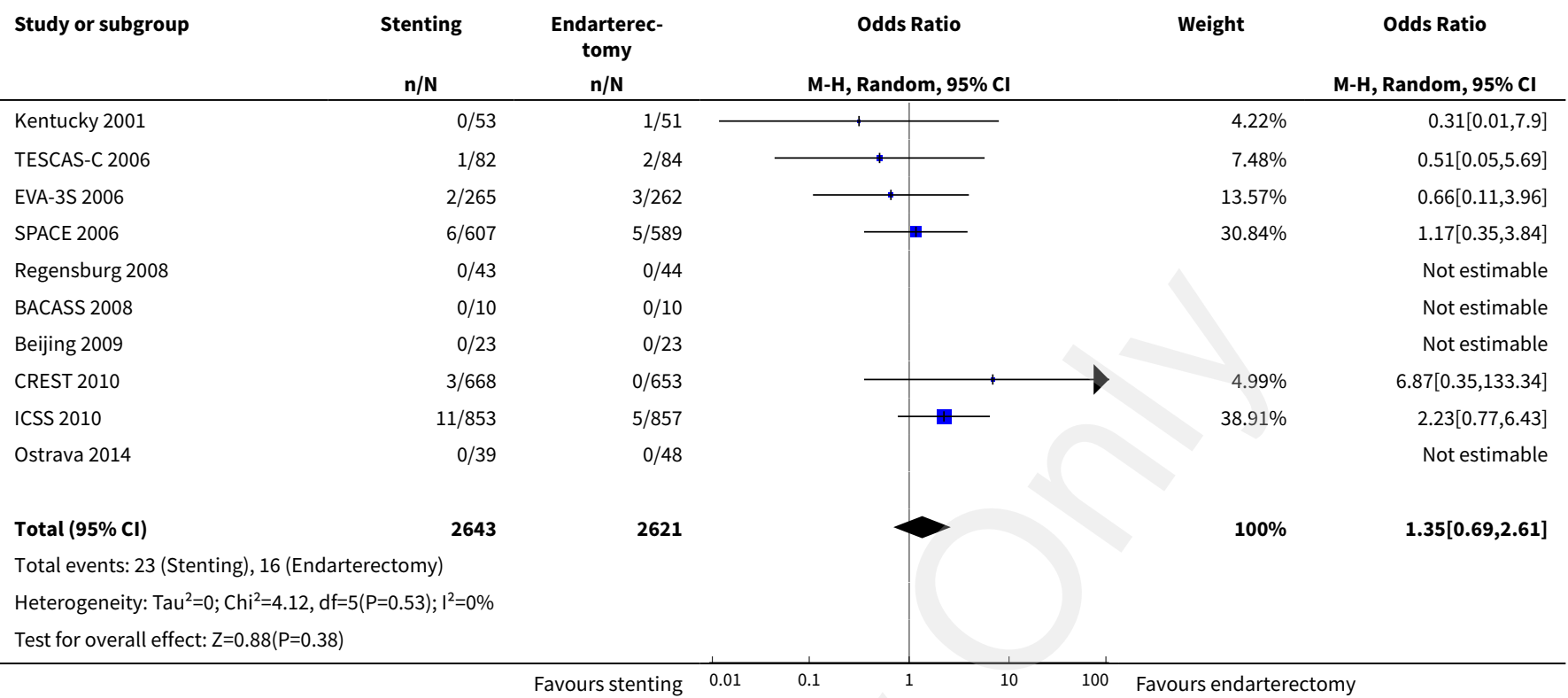

Analysis 1.7. Comparison 1 Stenting or endarterectomy for symptomatic carotid stenosis, Outcome 7 Any stroke between randomisation and 30 days after treatment.

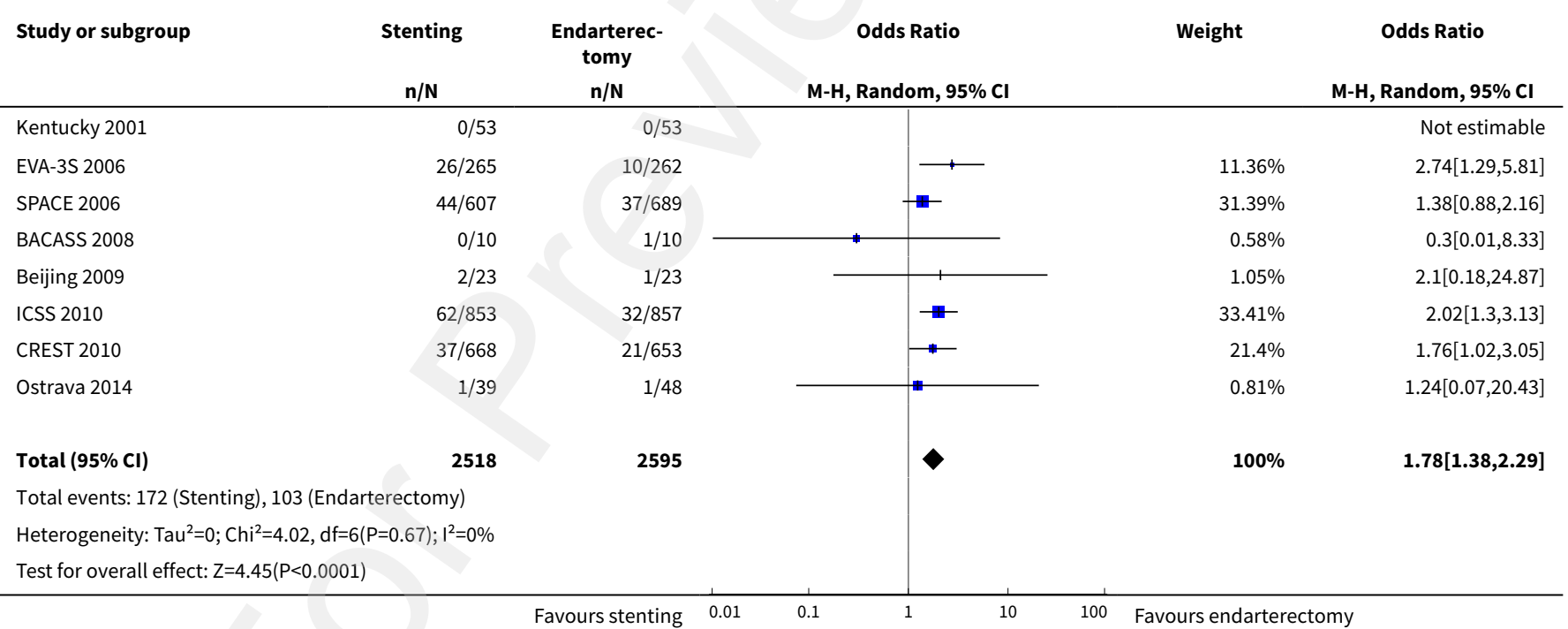


Analysis 1.8. Comparison 1 Stenting or endarterectomy for symptomatic carotid stenosis, Outcome 8 Fatal, major, or disabling stroke between randomisation and $\mathbf{3 0}$ days after treatment.

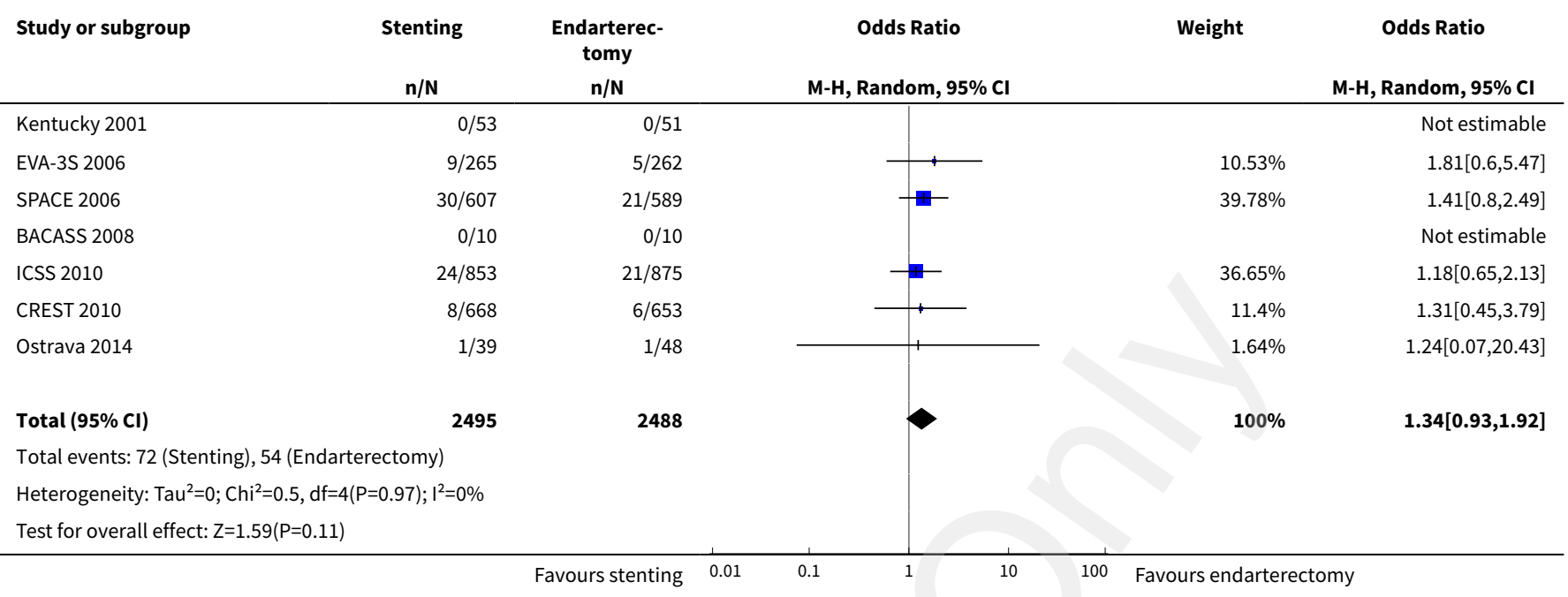

\section{Analysis 1.9. Comparison 1 Stenting or endarterectomy for symptomatic carotid stenosis, Outcome 9 Myocardial infarction between randomisation and 30 days after treatment.}

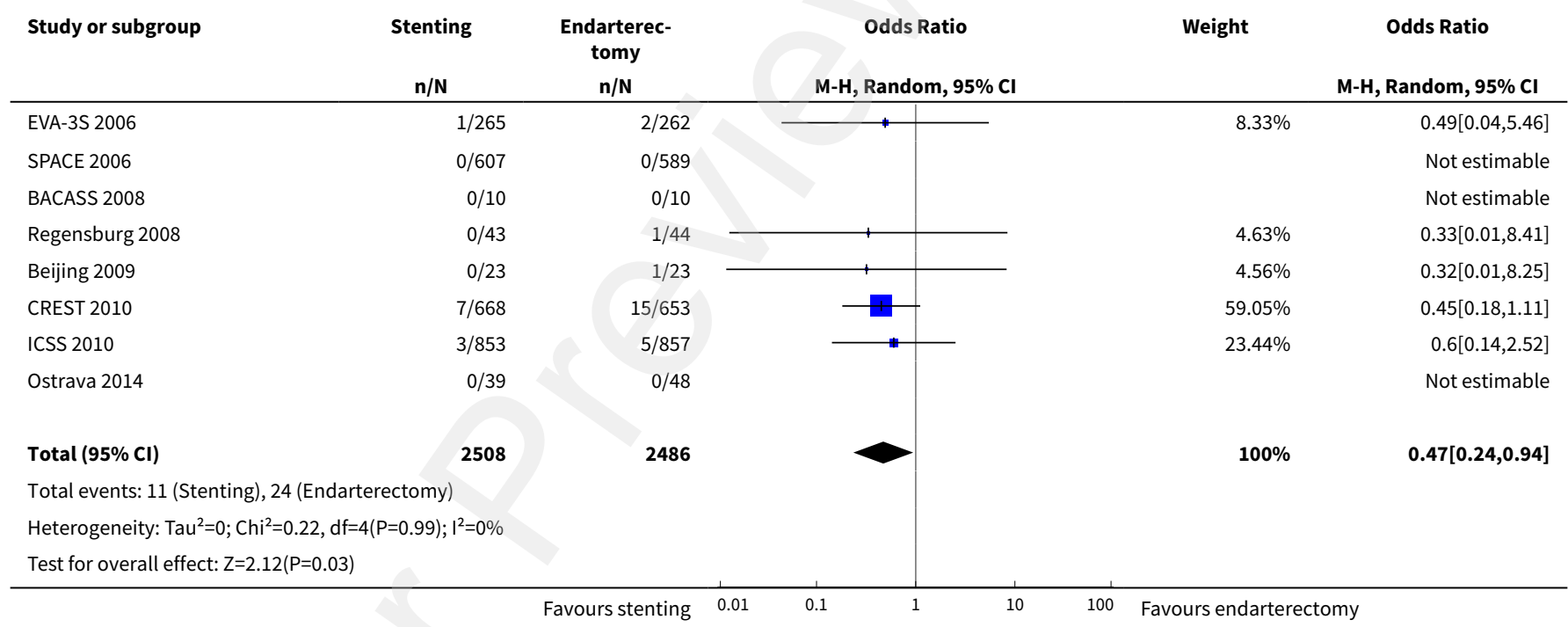

Analysis 1.10. Comparison 1 Stenting or endarterectomy for symptomatic carotid stenosis, Outcome 10 Death or any stroke or myocardial infarction between randomisation and $\mathbf{3 0}$ days after treatment.

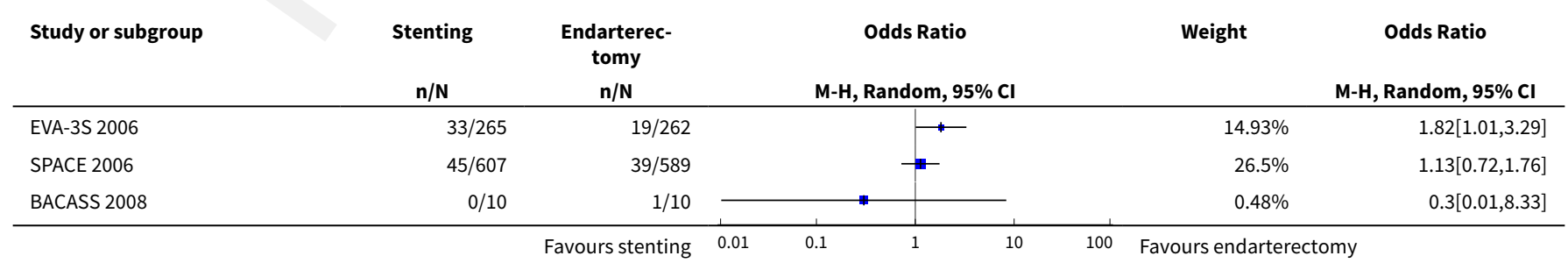




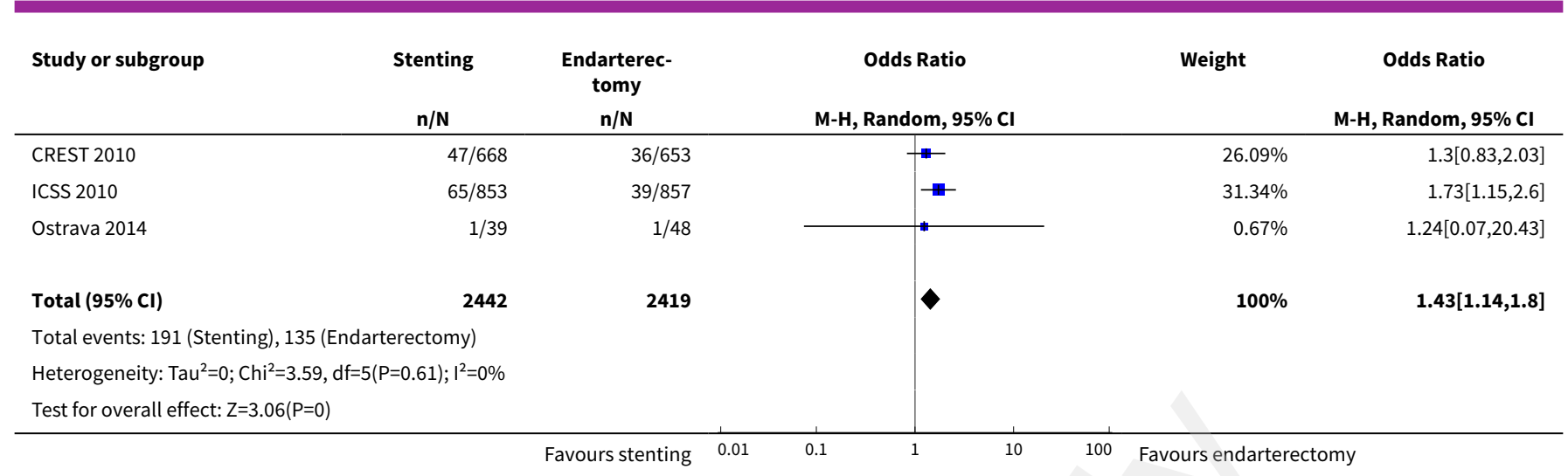

Analysis 1.11. Comparison 1 Stenting or endarterectomy for symptomatic carotid stenosis, Outcome 11 Primary combined safety and efficacy outcome: death or any stroke between randomisation and $\mathbf{3 0}$ days after treatment or ipsilateral stroke until the end of follow-up.

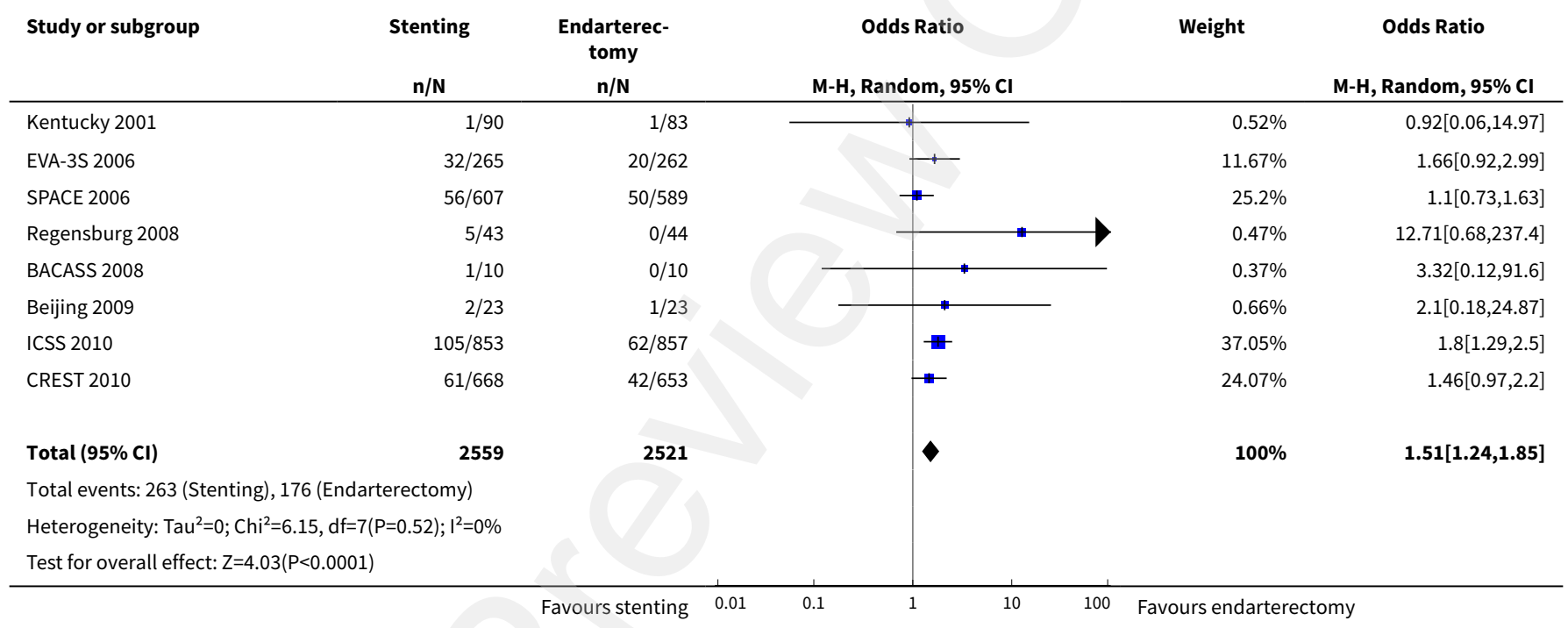

Analysis 1.12. Comparison 1 Stenting or endarterectomy for symptomatic carotid stenosis, Outcome 12 Death or any stroke between randomisation and end of follow-up.

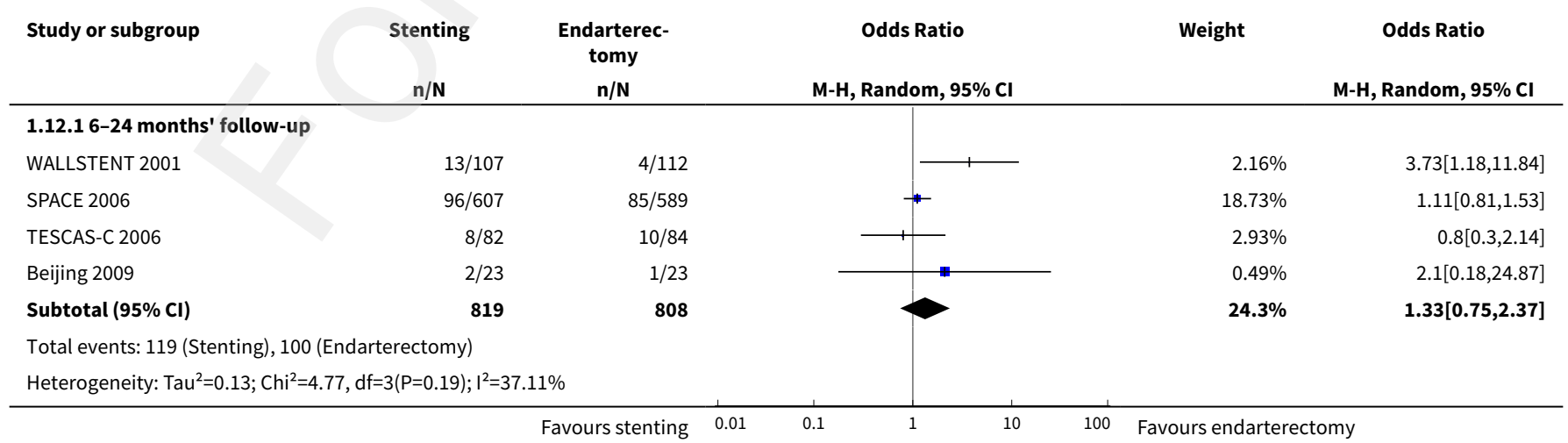




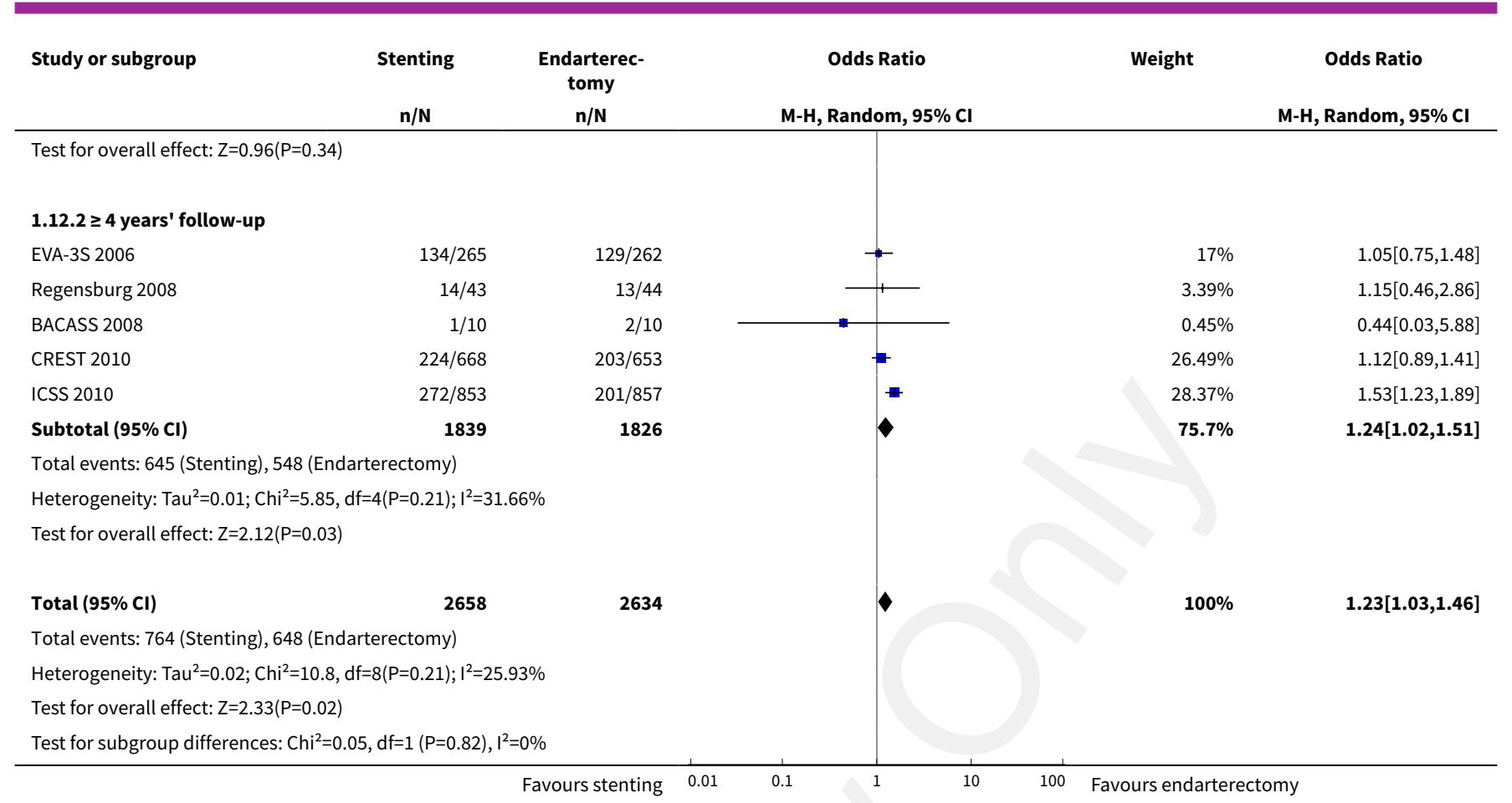

\section{Analysis 1.13. Comparison 1 Stenting or endarterectomy for symptomatic carotid stenosis, Outcome 13 Any stroke during follow-up (periprocedural events and people dying in the periprocedural period excluded).}

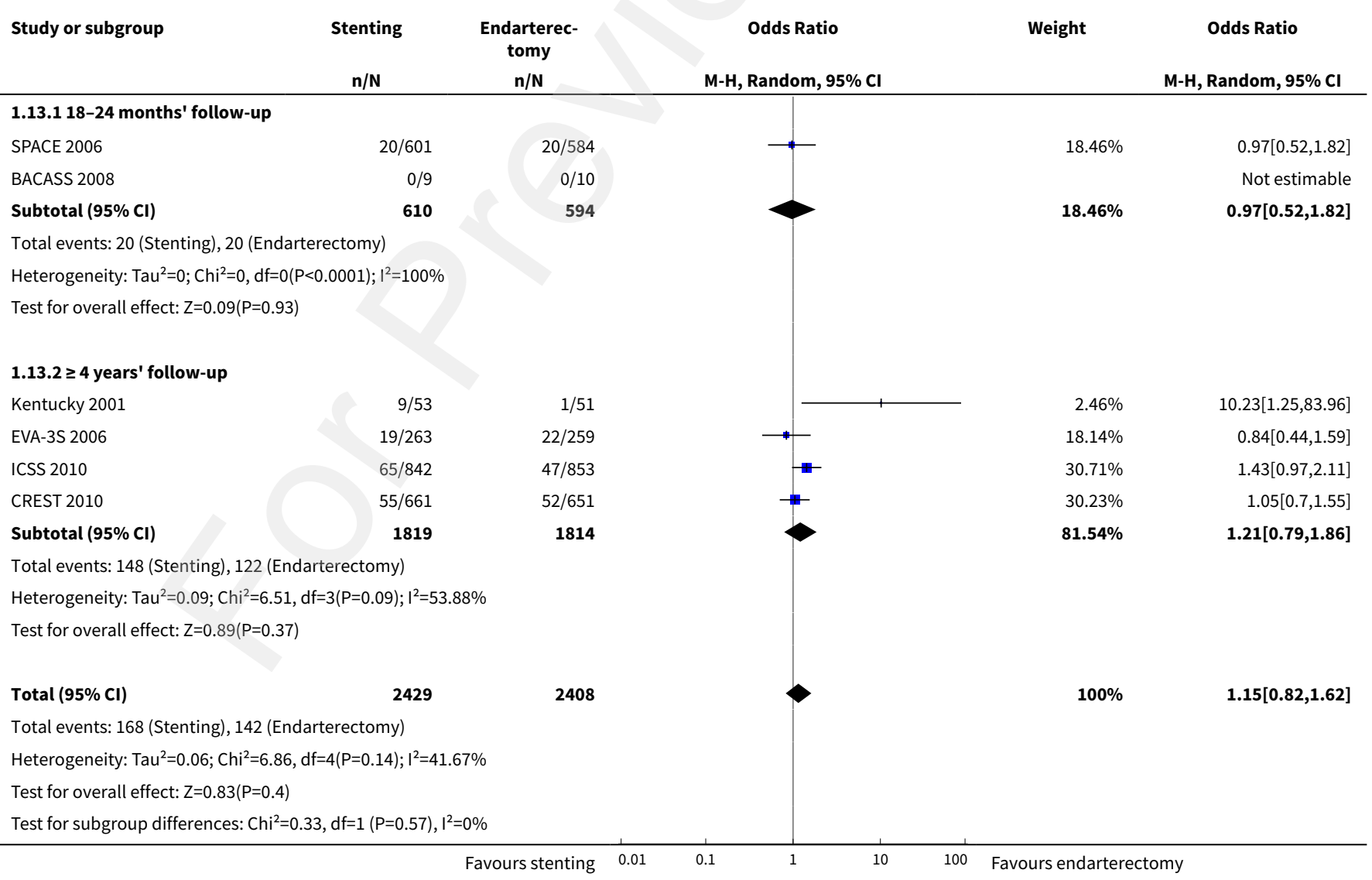


Analysis 1.14. Comparison 1 Stenting or endarterectomy for symptomatic carotid stenosis, Outcome 14 Ipsilateral stroke during follow-up (periprocedural events and people dying in the periprocedural period excluded).

\begin{tabular}{|c|c|c|c|c|c|}
\hline \multirow[t]{2}{*}{ Study or subgroup } & \multirow{2}{*}{$\begin{array}{c}\text { Stenting } \\
\mathbf{n} / \mathbf{N} \\
\end{array}$} & \multirow{2}{*}{$\begin{array}{c}\text { Endarterec- } \\
\text { tomy } \\
n / N \\
\end{array}$} & Odds Ratio & \multirow[t]{2}{*}{ Weight } & \multirow{2}{*}{$\begin{array}{c}\text { Odds Ratio } \\
\text { M-H, Random, } 95 \% \mathrm{Cl} \\
\end{array}$} \\
\hline & & & M-H, Random, 95\% Cl & & \\
\hline \multicolumn{3}{|c|}{ 1.14.1 18-24 months' follow-up } & & & \\
\hline SPACE 2006 & $11 / 601$ & $10 / 584$ & $\longrightarrow$ & $14.78 \%$ & $1.07[0.45,2.54]$ \\
\hline BACASS 2008 & $0 / 9$ & $0 / 10$ & & & Not estimable \\
\hline Subtotal $(95 \% \mathrm{Cl})$ & 610 & 594 & & $14.78 \%$ & $1.07[0.45,2.54]$ \\
\hline \multicolumn{6}{|c|}{ Heterogeneity: Not applicable } \\
\hline \multicolumn{6}{|c|}{ Test for overall effect: $Z=0.15(P=0.88)$} \\
\hline \multicolumn{6}{|c|}{ 1.14.2 $\geq 4$ years' follow-up } \\
\hline Kentucky 2001 & $1 / 53$ & $0 / 51$ & & $1.06 \%$ & $2.94[0.12,73.92]$ \\
\hline EVA-3S 2006 & $6 / 263$ & $8 / 259$ & & $9.59 \%$ & $0.73[0.25,2.14]$ \\
\hline CREST 2010 & $21 / 661$ & $21 / 651$ & & $29.19 \%$ & $0.98[0.53,1.82]$ \\
\hline Subtotal $(95 \% \mathrm{Cl})$ & 1819 & 1814 & & $85.22 \%$ & $1.05[0.73,1.5]$ \\
\hline \multicolumn{6}{|c|}{ Total events: 63 (Stenting), 60 (Endarterectomy) } \\
\hline \multicolumn{6}{|c|}{ Heterogeneity: $\operatorname{Tau}^{2}=0 ; \mathrm{Chi}^{2}=1, \mathrm{df}=3(\mathrm{P}=0.8) ; \mathrm{I}^{2}=0 \%$} \\
\hline \multicolumn{6}{|c|}{ Test for overall effect: $Z=0.26(P=0.8)$} \\
\hline Total $(95 \% \mathrm{Cl})$ & 2429 & 2408 & & $100 \%$ & $1.05[0.75,1.47]$ \\
\hline \multicolumn{6}{|c|}{ Total events: 74 (Stenting), 70 (Endarterectomy) } \\
\hline \multicolumn{6}{|c|}{ Heterogeneity: $\operatorname{Tau}^{2}=0 ; \mathrm{Chi}^{2}=1, \mathrm{df}=4(\mathrm{P}=0.91) ; \mathrm{I}^{2}=0 \%$} \\
\hline \multicolumn{6}{|c|}{ Test for overall effect: $Z=0.3(P=0.77)$} \\
\hline \multicolumn{6}{|c|}{ Test for subgroup differences: $\mathrm{Chi}^{2}=0, \mathrm{df}=1(\mathrm{P}=0.97), \mathrm{I}^{2}=0 \%$} \\
\hline & & ours stentin & 0.1 & $x_{1}$ & \\
\hline
\end{tabular}

Analysis 1.15. Comparison 1 Stenting or endarterectomy for symptomatic carotid stenosis, Outcome 15 Death or any stroke or myocardial infarction between randomisation and $\mathbf{3 0}$ days after treatment or ipsilateral stroke until end of follow-up.

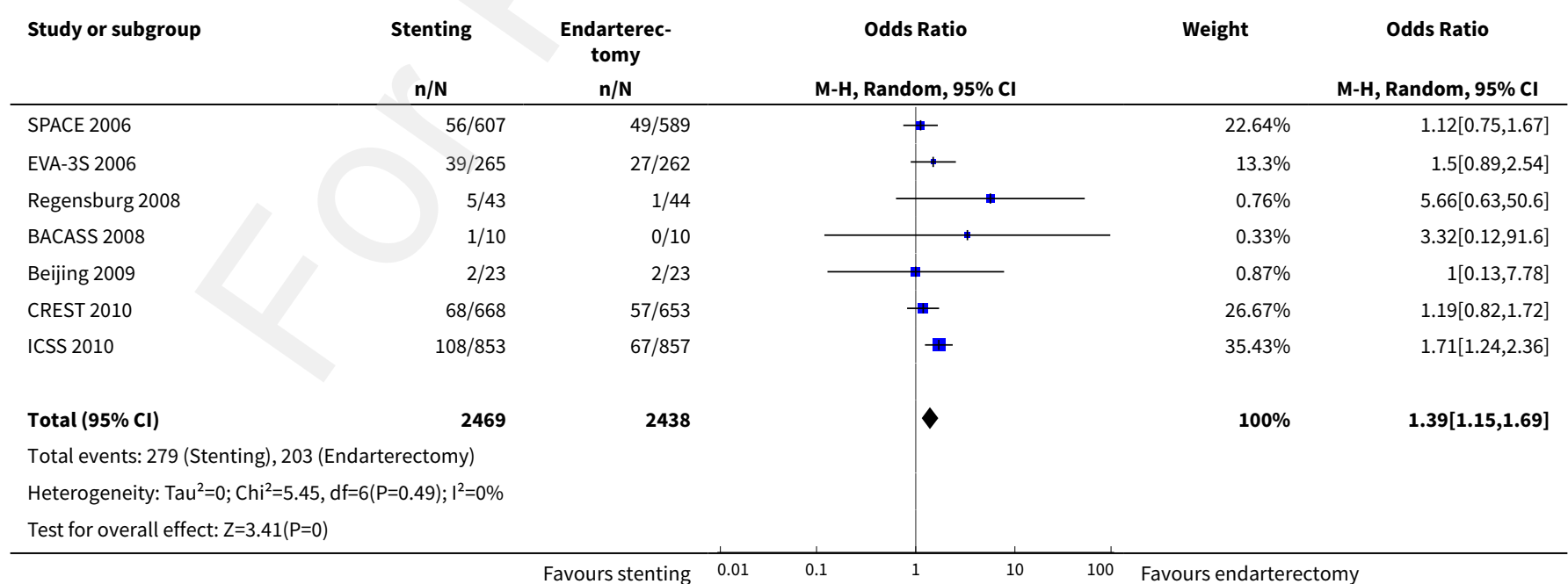


Comparison 2. Stenting or endarterectomy for asymptomatic carotid stenosis

\begin{tabular}{|c|c|c|c|c|}
\hline Outcome or subgroup title & $\begin{array}{l}\text { No. of } \\
\text { studies }\end{array}$ & $\begin{array}{l}\text { No. of } \\
\text { partici- } \\
\text { pants }\end{array}$ & Statistical method & Effect size \\
\hline $\begin{array}{l}1 \text { Death or any stroke between randomisation and } 30 \text { days af- } \\
\text { ter treatment }\end{array}$ & 7 & 3378 & $\begin{array}{l}\text { Odds Ratio (M-H, Ran- } \\
\text { dom, } 95 \% \mathrm{Cl})\end{array}$ & $\begin{array}{l}1.72[1.00 \\
2.97]\end{array}$ \\
\hline $\begin{array}{l}2 \text { Death or major or disabling stroke between randomisation } \\
\text { and } 30 \text { days after treatment }\end{array}$ & 2 & 2601 & $\begin{array}{l}\text { Odds Ratio (M-H, Ran- } \\
\text { dom, } 95 \% \mathrm{Cl})\end{array}$ & $\begin{array}{l}1.54[0.39 \\
6.11]\end{array}$ \\
\hline $\begin{array}{l}3 \text { Myocardial infarction between randomisation and } 30 \text { days } \\
\text { after treatment }\end{array}$ & 6 & 2945 & $\begin{array}{l}\text { Odds Ratio (M-H, Ran- } \\
\text { dom, } 95 \% \mathrm{Cl})\end{array}$ & $\begin{array}{l}0.53[0.24, \\
1.15]\end{array}$ \\
\hline $\begin{array}{l}4 \text { Death or any stroke or myocardial infarction between ran- } \\
\text { domisation and } 30 \text { days after treatment }\end{array}$ & 6 & 2978 & $\begin{array}{l}\text { Odds Ratio (M-H, Ran- } \\
\text { dom, } 95 \% \mathrm{Cl})\end{array}$ & $\begin{array}{l}1.16[0.73, \\
1.85]\end{array}$ \\
\hline $\begin{array}{l}5 \text { Death or any stroke between randomisation and } 30 \text { days af- } \\
\text { ter treatment or ipsilateral stroke until end of follow-up }\end{array}$ & 6 & 3315 & $\begin{array}{l}\text { Odds Ratio (M-H, Ran- } \\
\text { dom, } 95 \% \mathrm{Cl})\end{array}$ & $\begin{array}{l}1.27[0.87 \\
1.84]\end{array}$ \\
\hline $\begin{array}{l}6 \text { Death or any stroke or myocardial infarction between ran- } \\
\text { domisation and } 30 \text { days after treatment or ipsilateral stroke } \\
\text { until end of follow-up }\end{array}$ & 5 & 2882 & $\begin{array}{l}\text { Odds Ratio (M-H, Ran- } \\
\text { dom, } 95 \% \mathrm{Cl})\end{array}$ & $\begin{array}{l}1.10 \text { [0.76, } \\
1.59]\end{array}$ \\
\hline
\end{tabular}

\section{Analysis 2.1. Comparison 2 Stenting or endarterectomy for asymptomatic carotid stenosis, Outcome 1 Death or any stroke between randomisation and 30 days after treatment.}

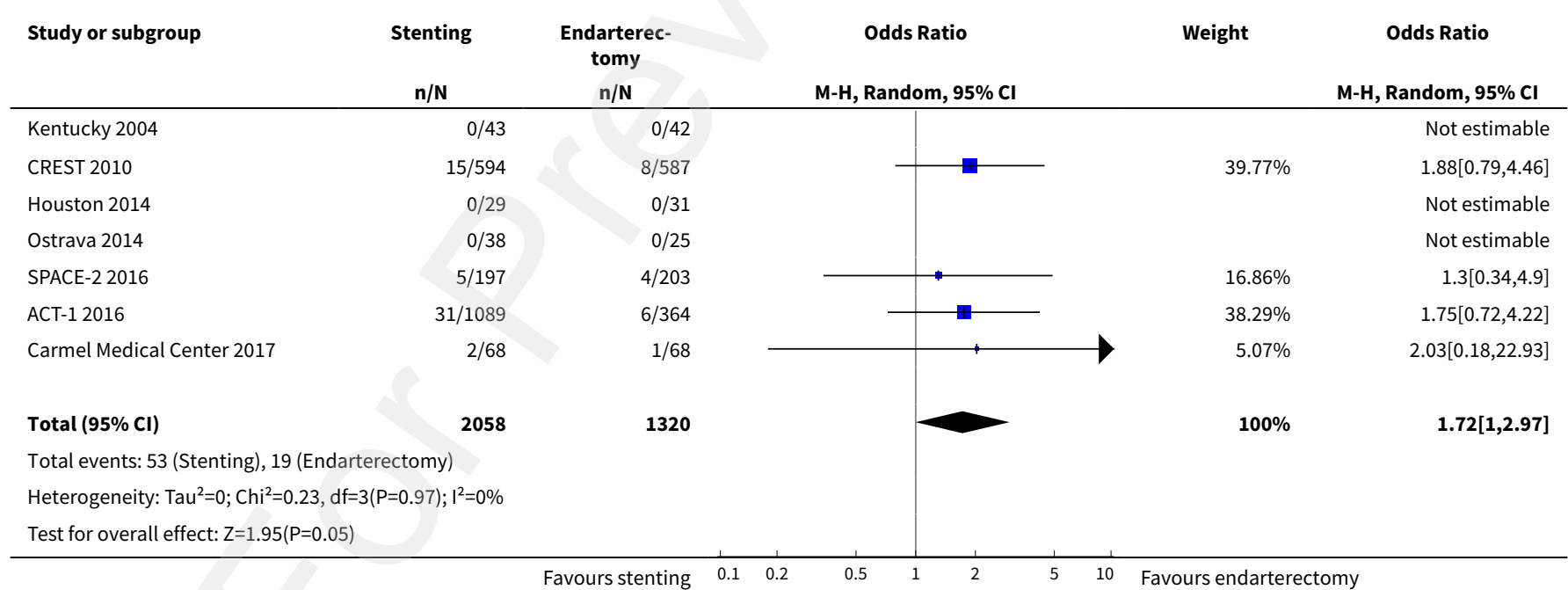


Analysis 2.2. Comparison 2 Stenting or endarterectomy for asymptomatic carotid stenosis, Outcome 2 Death or major or disabling stroke between randomisation and 30 days after treatment.

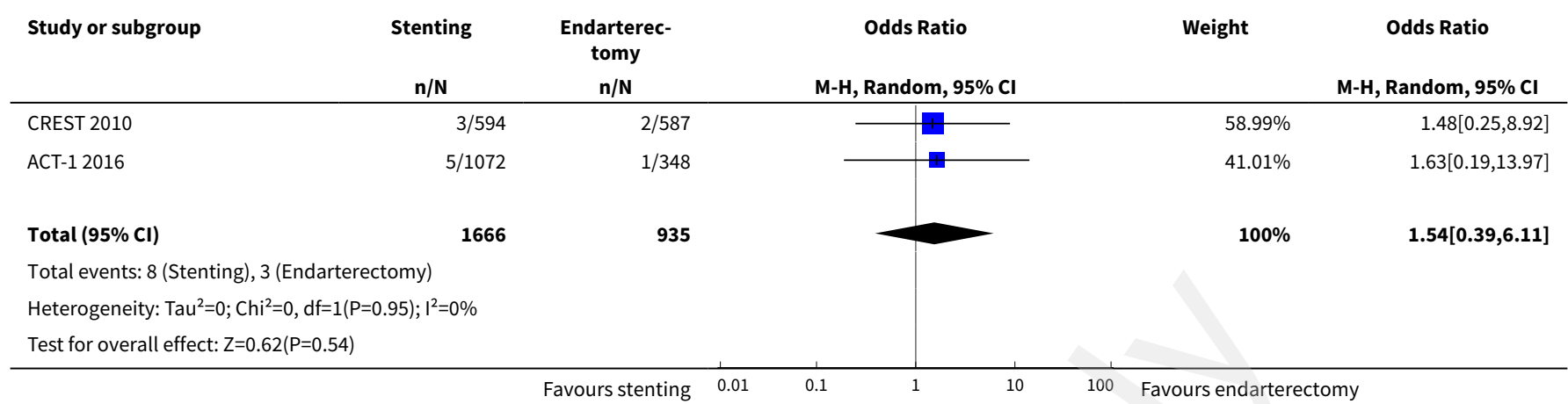

Analysis 2.3. Comparison 2 Stenting or endarterectomy for asymptomatic carotid stenosis, Outcome 3 Myocardial infarction between randomisation and 30 days after treatment.

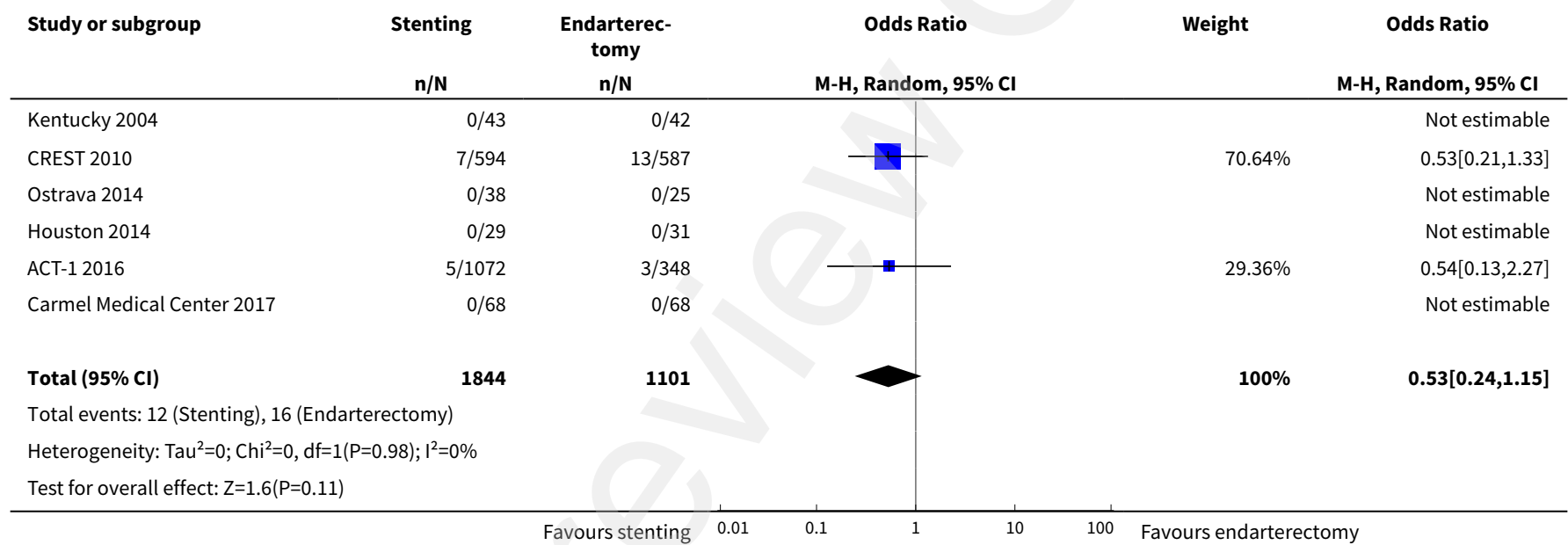

Analysis 2.4. Comparison 2 Stenting or endarterectomy for asymptomatic carotid stenosis, Outcome 4 Death or any stroke or myocardial infarction between randomisation and 30 days after treatment.

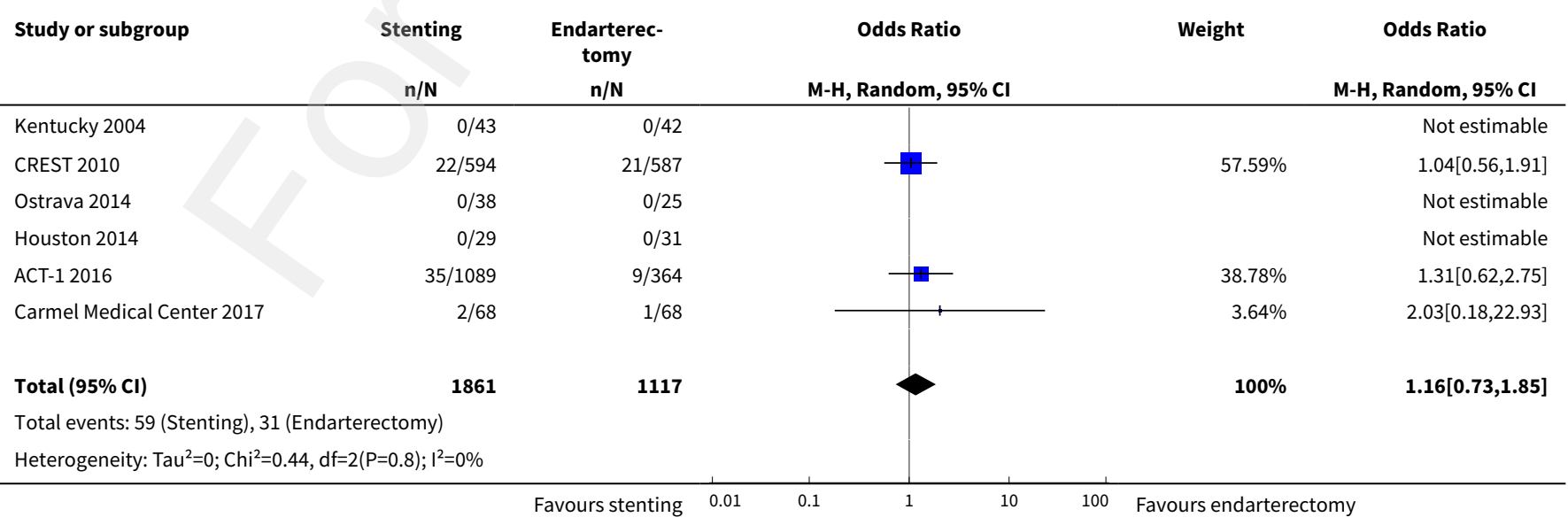




\begin{tabular}{|c|c|c|c|c|c|c|c|c|}
\hline \multirow[t]{2}{*}{ Study or subgroup } & \multirow{2}{*}{$\begin{array}{c}\text { Stenting } \\
n / N\end{array}$} & \multirow{2}{*}{$\begin{array}{c}\text { Endarterec- } \\
\text { tomy } \\
n / N\end{array}$} & \multicolumn{3}{|c|}{ Odds Ratio } & \multirow{2}{*}{\multicolumn{2}{|c|}{ Weight }} & \multirow{2}{*}{$\begin{array}{c}\text { Odds Ratio } \\
\text { M-H, Random, } 95 \% \mathrm{CI}\end{array}$} \\
\hline & & & \multicolumn{3}{|c|}{ M-H, Random, $95 \% \mathrm{CI}$} & & & \\
\hline \multicolumn{2}{|l|}{ Test for overall effect: $Z=0.64(P=0.52)$} & & & & & & & \\
\hline & & Favours stenting & 0.01 & 0.1 & 10 & 100 & Favours enda & omy \\
\hline
\end{tabular}

Analysis 2.5. Comparison 2 Stenting or endarterectomy for asymptomatic carotid stenosis, Outcome 5 Death or any stroke between randomisation and $\mathbf{3 0}$ days after treatment or ipsilateral stroke until end of follow-up.

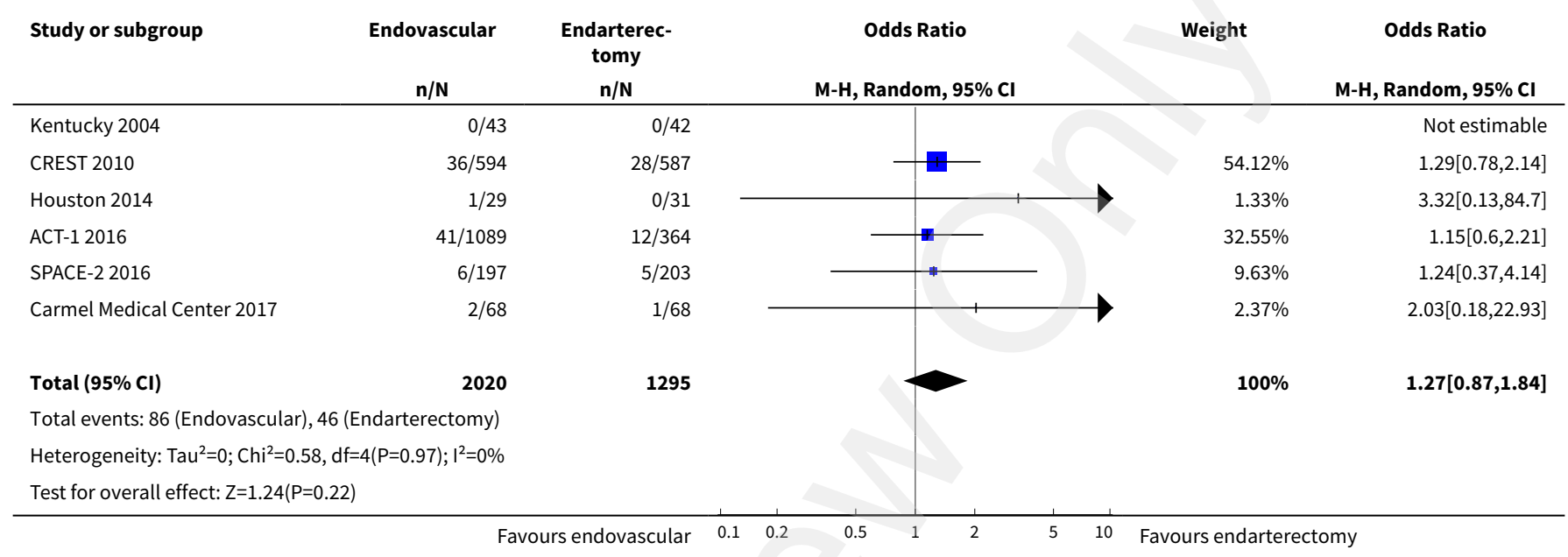

Analysis 2.6. Comparison 2 Stenting or endarterectomy for asymptomatic carotid stenosis, Outcome 6 Death or any stroke or myocardial infarction between randomisation and $\mathbf{3 0}$ days after treatment or ipsilateral stroke until end of follow-up.

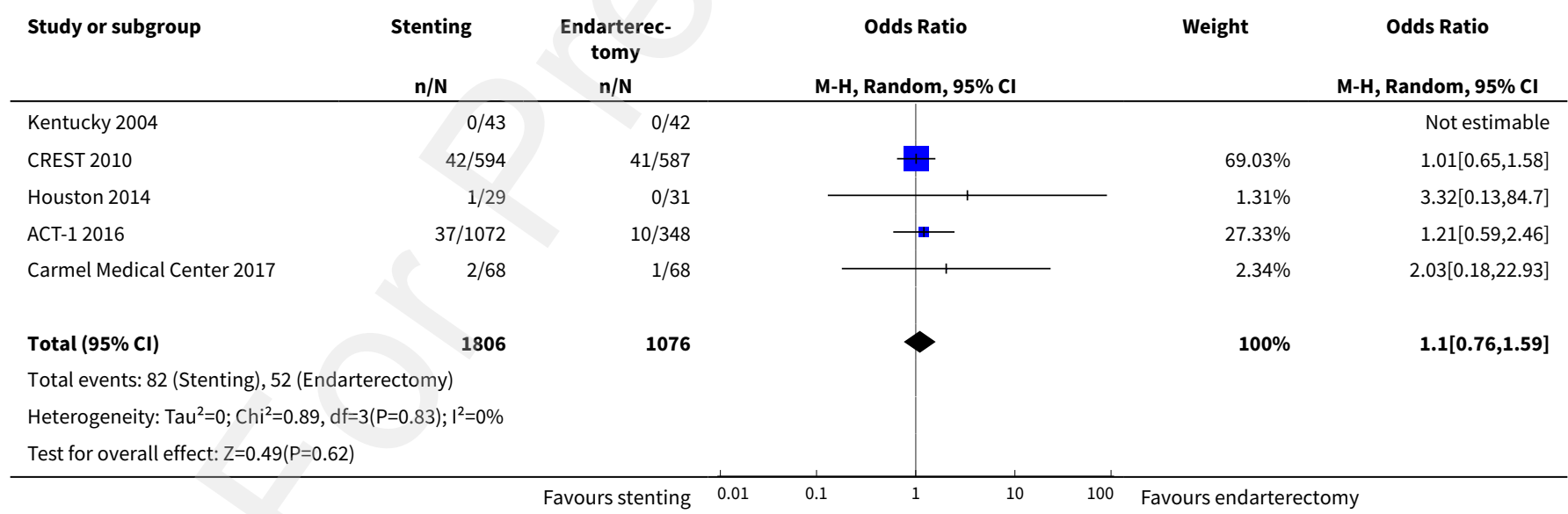


Comparison 3. Stenting or endarterectomy in people with elevated surgical risk

\begin{tabular}{|c|c|c|c|c|}
\hline Outcome or subgroup title & $\begin{array}{l}\text { No. of } \\
\text { studies }\end{array}$ & $\begin{array}{l}\text { No. of } \\
\text { partici- } \\
\text { pants }\end{array}$ & Statistical method & Effect size \\
\hline $\begin{array}{l}1 \text { Death or any stroke between randomisation and } 30 \text { days } \\
\text { after treatment }\end{array}$ & 2 & 397 & $\begin{array}{l}\text { Odds Ratio (M-H, Random, } \\
95 \% \mathrm{Cl})\end{array}$ & $\begin{array}{l}0.95[0.39 \\
2.28]\end{array}$ \\
\hline $\begin{array}{l}2 \text { Fatal, major, or disabling stroke between randomisation } \\
\text { and } 30 \text { days after treatment }\end{array}$ & 1 & 334 & $\begin{array}{l}\text { Odds Ratio (M-H, Random, } \\
95 \% \mathrm{Cl})\end{array}$ & $\begin{array}{l}0.66[0.11 \\
4.02]\end{array}$ \\
\hline $\begin{array}{l}3 \text { Death of any cause between randomisation and } 30 \text { days } \\
\text { after treatment }\end{array}$ & 2 & 397 & $\begin{array}{l}\text { Odds Ratio (M-H, Random, } \\
95 \% \mathrm{Cl})\end{array}$ & $\begin{array}{l}0.47[0.10, \\
2.15]\end{array}$ \\
\hline $\begin{array}{l}4 \text { Any stroke between randomisation and } 30 \text { days after } \\
\text { treatment }\end{array}$ & 2 & 397 & $\begin{array}{l}\text { Odds Ratio (M-H, Random, } \\
95 \% \mathrm{Cl})\end{array}$ & $\begin{array}{l}1.40[0.48, \\
4.14]\end{array}$ \\
\hline $\begin{array}{l}5 \text { Myocardial infarction between randomisation and } 30 \\
\text { days after treatment }\end{array}$ & 2 & 397 & $\begin{array}{l}\text { Odds Ratio (M-H, Random, } \\
95 \% \mathrm{Cl})\end{array}$ & $\begin{array}{l}0.39[0.12 \\
1.25]\end{array}$ \\
\hline $\begin{array}{l}6 \text { Death or any stroke or myocardial infarction between ran- } \\
\text { domisation and } 30 \text { days after treatment }\end{array}$ & 2 & 397 & $\begin{array}{l}\text { Odds Ratio (M-H, Random, } \\
95 \% \mathrm{Cl})\end{array}$ & $\begin{array}{l}0.66[0.33 \\
1.34]\end{array}$ \\
\hline $\begin{array}{l}7 \text { Death or any stroke between randomisation and } 30 \text { days } \\
\text { after treatment or ipsilateral stroke until end of follow-up }\end{array}$ & 2 & 397 & $\begin{array}{l}\text { Odds Ratio (M-H, Random, } \\
95 \% \mathrm{Cl})\end{array}$ & $\begin{array}{l}1.09[0.58, \\
2.06]\end{array}$ \\
\hline $\begin{array}{l}8 \text { Death or any stroke or myocardial infarction between ran- } \\
\text { domisation and } 30 \text { days after treatment or ipsilateral stroke } \\
\text { until end of follow-up }\end{array}$ & 2 & 397 & $\begin{array}{l}\text { Odds Ratio (M-H, Random, } \\
95 \% \mathrm{Cl})\end{array}$ & $\begin{array}{l}0.84[0.48, \\
1.47]\end{array}$ \\
\hline $\begin{array}{l}9 \text { Any stroke during follow-up (periprocedural events and } \\
\text { people dying in the periprocedural period excluded) }\end{array}$ & 2 & 397 & $\begin{array}{l}\text { Odds Ratio (M-H, Random, } \\
95 \% \mathrm{Cl})\end{array}$ & $\begin{array}{l}1.02[0.49 \\
2.10]\end{array}$ \\
\hline
\end{tabular}

Analysis 3.1. Comparison 3 Stenting or endarterectomy in people with elevated surgical risk, Outcome 1 Death or any stroke between randomisation and 30 days after treatment.

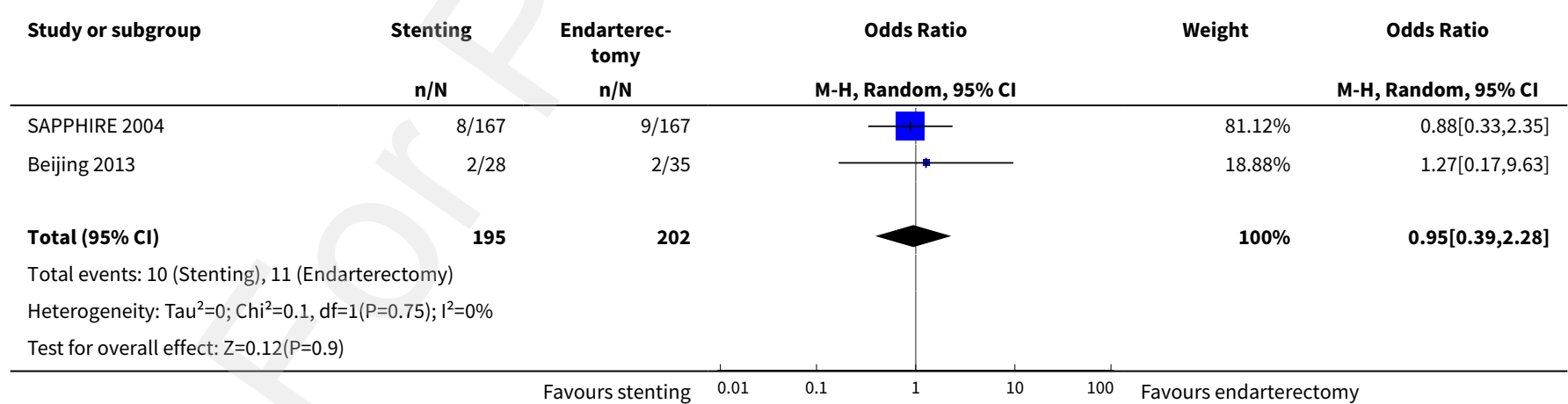


Analysis 3.2. Comparison 3 Stenting or endarterectomy in people with elevated surgical risk, Outcome 2 Fatal, major, or disabling stroke between randomisation and $\mathbf{3 0}$ days after treatment.

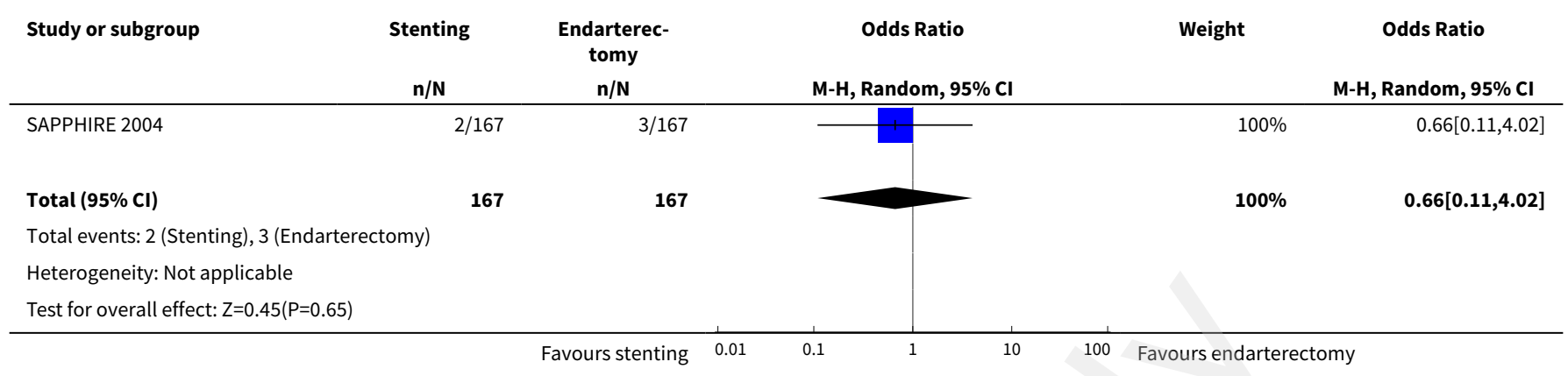

Analysis 3.3. Comparison 3 Stenting or endarterectomy in people with elevated surgical risk, Outcome 3 Death of any cause between randomisation and 30 days after treatment.

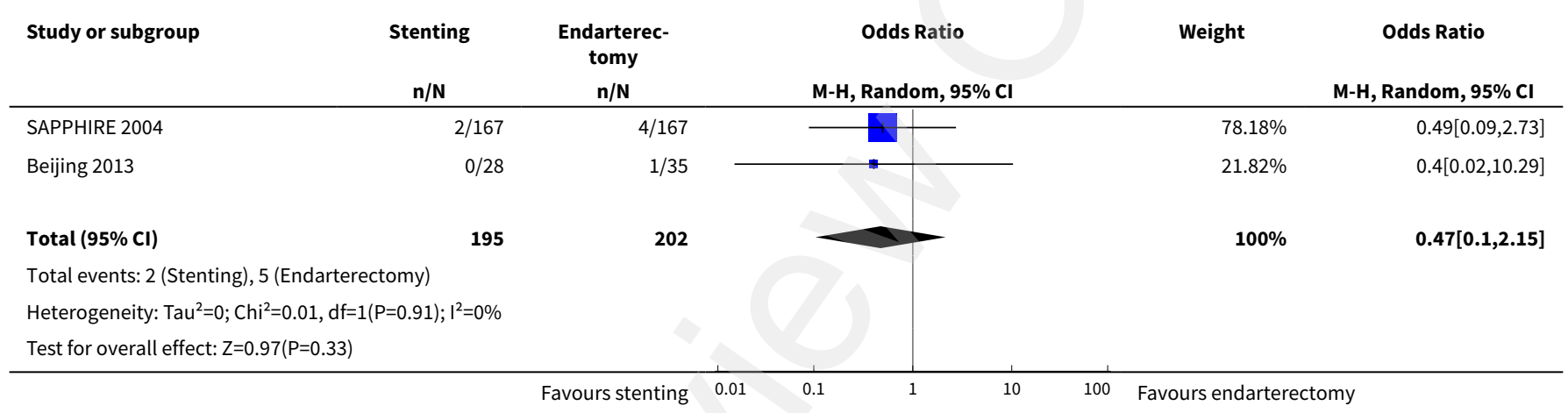

Analysis 3.4. Comparison 3 Stenting or endarterectomy in people with elevated surgical risk, Outcome 4 Any stroke between randomisation and 30 days after treatment.

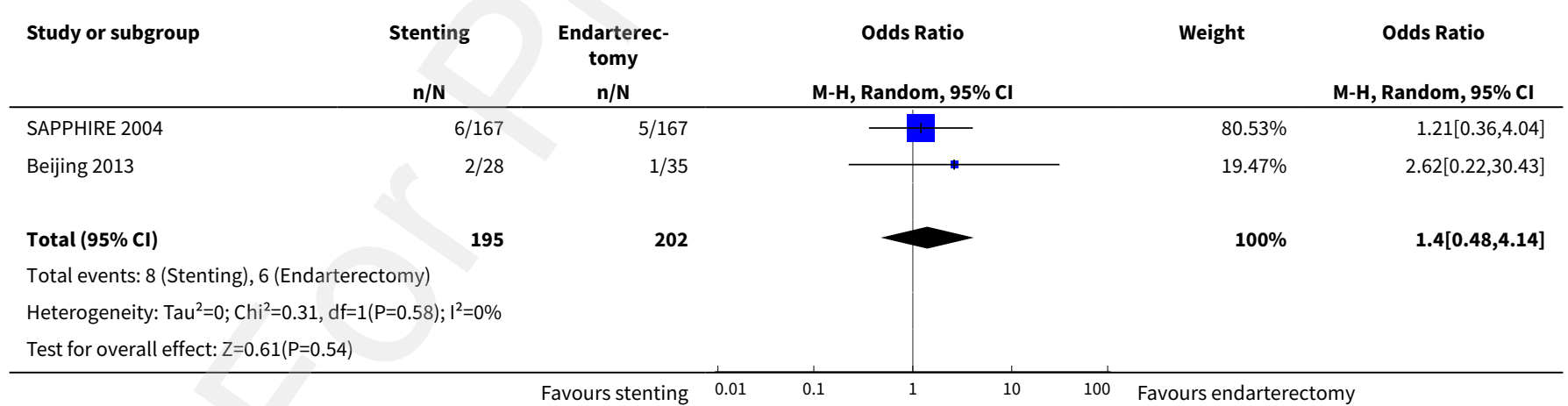


Analysis 3.5. Comparison 3 Stenting or endarterectomy in people with elevated surgical risk, Outcome 5 Myocardial infarction between randomisation and 30 days after treatment.

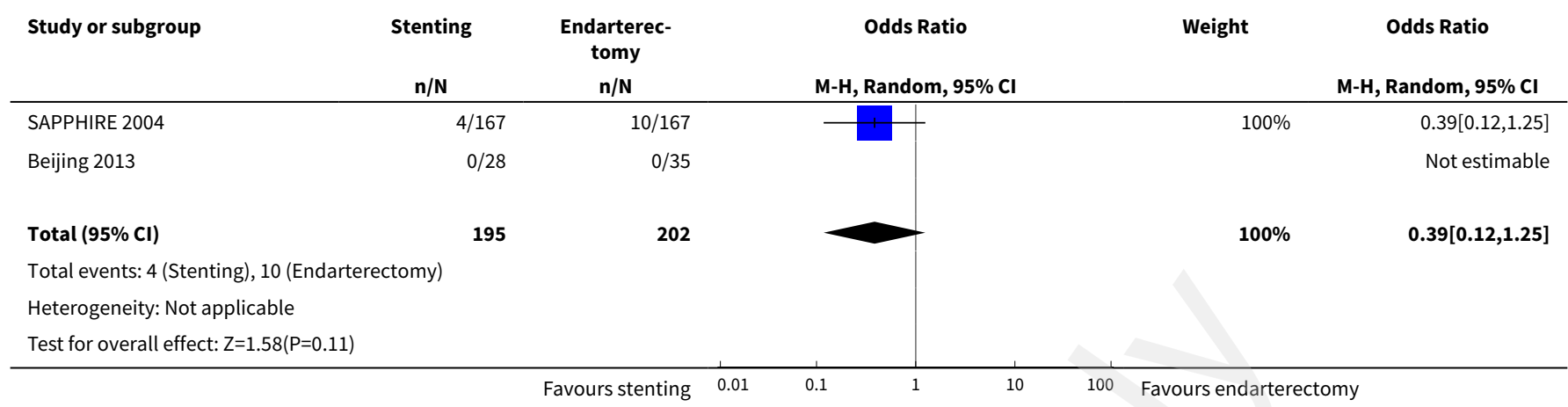

Analysis 3.6. Comparison 3 Stenting or endarterectomy in people with elevated surgical risk, Outcome 6 Death or any stroke or myocardial infarction between randomisation and 30 days after treatment.

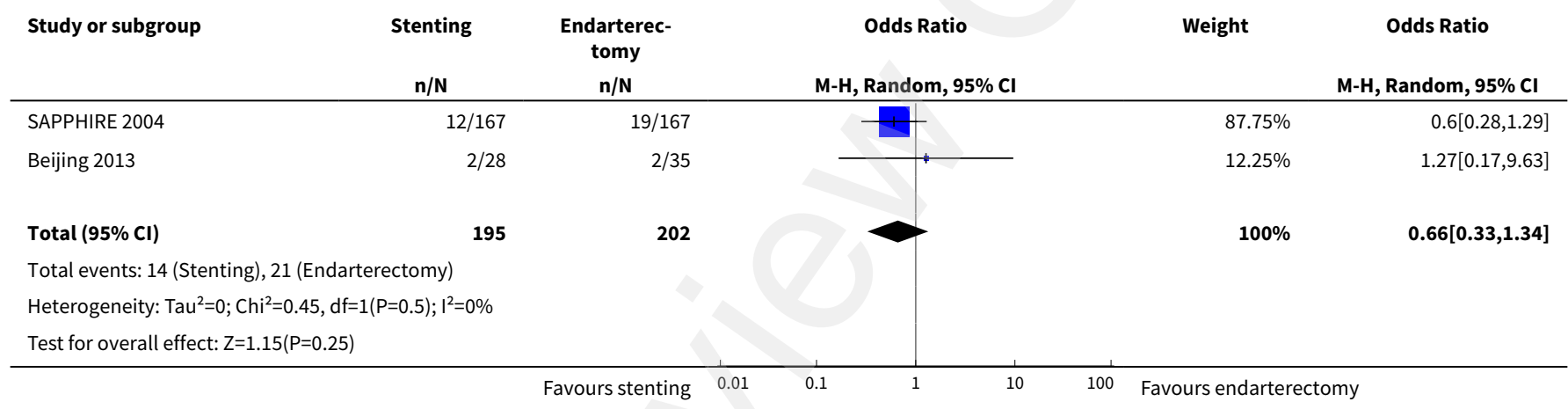

Analysis 3.7. Comparison 3 Stenting or endarterectomy in people with elevated surgical risk, Outcome 7 Death or any stroke between randomisation and $\mathbf{3 0}$ days after treatment or ipsilateral stroke until end of follow-up.

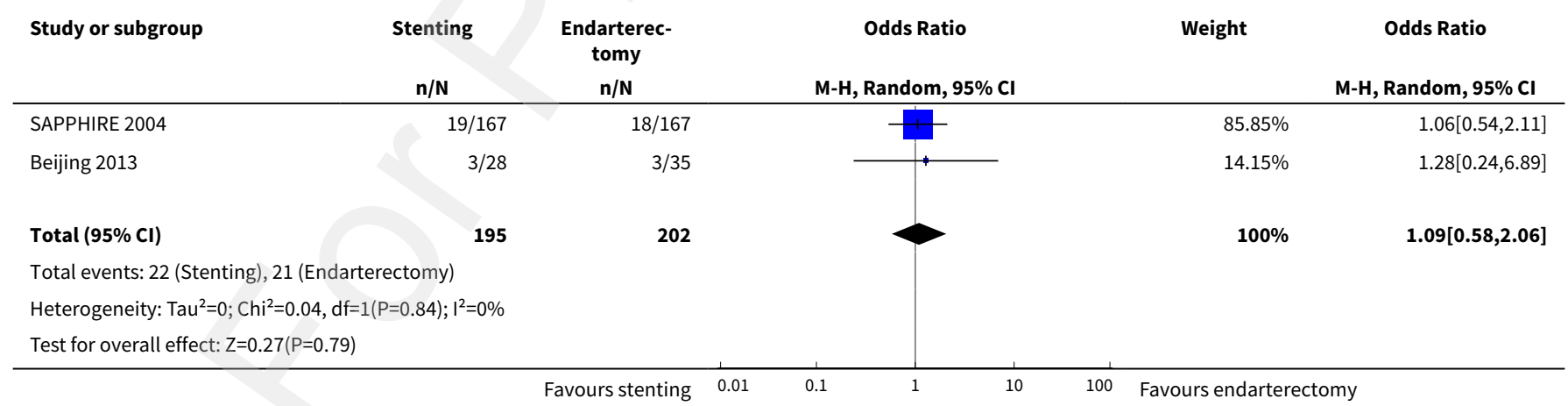


Analysis 3.8. Comparison 3 Stenting or endarterectomy in people with elevated surgical risk, Outcome 8 Death or any stroke or myocardial infarction between randomisation and $\mathbf{3 0}$ days after treatment or ipsilateral stroke until end of follow-up.

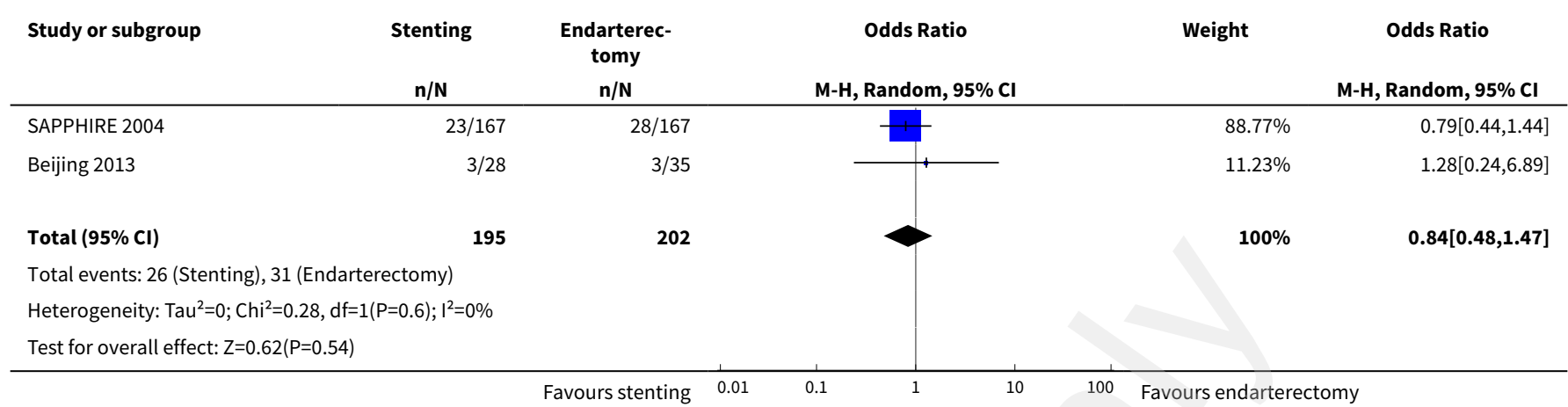

Analysis 3.9. Comparison 3 Stenting or endarterectomy in people with elevated surgical risk, Outcome 9 Any stroke during follow-up (periprocedural events and people dying in the periprocedural period excluded).

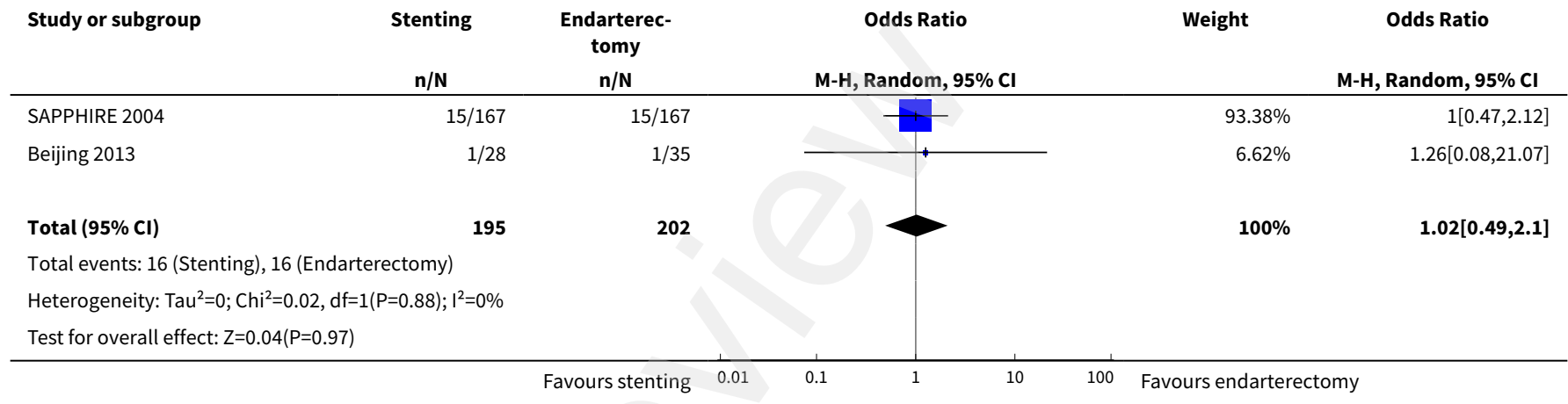

Comparison 4. Restenosis in endovascular treatment or endarterectomy for symptomatic or asymptomatic carotid stenosis

\begin{tabular}{llllll}
\hline Outcome or subgroup title & $\begin{array}{l}\text { No. of } \\
\text { studies }\end{array}$ & $\begin{array}{l}\text { No. of } \\
\text { partici- } \\
\text { pants }\end{array}$ & Statistical method & Effect size \\
\hline 1 Severe $(\geq 70 \%)$ restenosis during follow-up & 9 & 5744 & $\begin{array}{l}\text { Odds Ratio (M-H, Random, 95\% } \\
\text { Cl) }\end{array}$ & $1.21[0.76,1.93]$ \\
\hline $\begin{array}{l}2 \text { Moderate }(\geq 50 \%) \text { or severe }(\geq 70 \%) \text { restenosis } \\
\text { during follow-up }\end{array}$ & 4 & 2115 & $\begin{array}{l}\text { Odds Ratio (M-H, Random, 95\% } \\
\text { Cl) }\end{array}$ & $2.00[1.12,3.60]$ \\
\hline
\end{tabular}


Analysis 4.1. Comparison 4 Restenosis in endovascular treatment or endarterectomy for symptomatic or asymptomatic carotid stenosis, Outcome 1 Severe $(\geq 70 \%)$ restenosis during follow-up.

\begin{tabular}{|c|c|c|c|c|c|}
\hline \multirow[t]{2}{*}{ Study or subgroup } & Stenting & \multirow{2}{*}{$\begin{array}{c}\text { Endarterec- } \\
\text { tomy } \\
n / N\end{array}$} & \multirow{2}{*}{$\begin{array}{c}\text { Odds Ratio } \\
\text { M-H, Random, } 95 \% \mathrm{CI}\end{array}$} & \multirow[t]{2}{*}{ Weight } & \multirow{2}{*}{$\begin{array}{c}\text { Odds Ratio } \\
\text { M-H, Random, } 95 \% \mathrm{Cl}\end{array}$} \\
\hline & $\mathrm{n} / \mathrm{N}$ & & & & \\
\hline EVA-3S 2006 & $7 / 247$ & $12 / 257$ & $\longrightarrow+$ & $13.87 \%$ & $0.6[0.23,1.54]$ \\
\hline SPACE 2006 & $54 / 607$ & $23 / 589$ & $\rightarrow$ & $23.66 \%$ & $2.4[1.45,3.97]$ \\
\hline Regensburg 2008 & $6 / 32$ & $0 / 29$ & & $2.36 \%$ & $14.47[0.78,269.37]$ \\
\hline BACASS 2008 & $0 / 8$ & $0 / 9$ & & & Not estimable \\
\hline ICSS 2010 & $72 / 737$ & $62 / 793$ & - & $27.4 \%$ & $1.28[0.89,1.82]$ \\
\hline CREST 2010 & $58 / 1086$ & $62 / 1105$ & & $27.08 \%$ & $0.95[0.66,1.37]$ \\
\hline Beijing 2013 & $0 / 28$ & $1 / 35$ & & $1.95 \%$ & $0.4[0.02,10.29]$ \\
\hline Carmel Medical Center 2017 & $1 / 68$ & $3 / 68$ & & $3.68 \%$ & $0.32[0.03,3.19]$ \\
\hline Total $(95 \% \mathrm{Cl})$ & 2836 & 2908 & & $100 \%$ & $1.21[0.76,1.93]$ \\
\hline \multicolumn{6}{|c|}{ Heterogeneity: $\mathrm{Tau}^{2}=0.17 ; \mathrm{Chi}^{2}=15.56, \mathrm{df}=6(\mathrm{P}=0.02) ; \mathrm{I}^{2}=61.44 \%$} \\
\hline Test for overall effect: $Z=0.81$ & & & & & \\
\hline
\end{tabular}

Analysis 4.2. Comparison 4 Restenosis in endovascular treatment or endarterectomy for symptomatic or asymptomatic carotid stenosis, Outcome 2 Moderate $(\geq 50 \%)$ or severe $(\geq 70 \%)$ restenosis during follow-up.

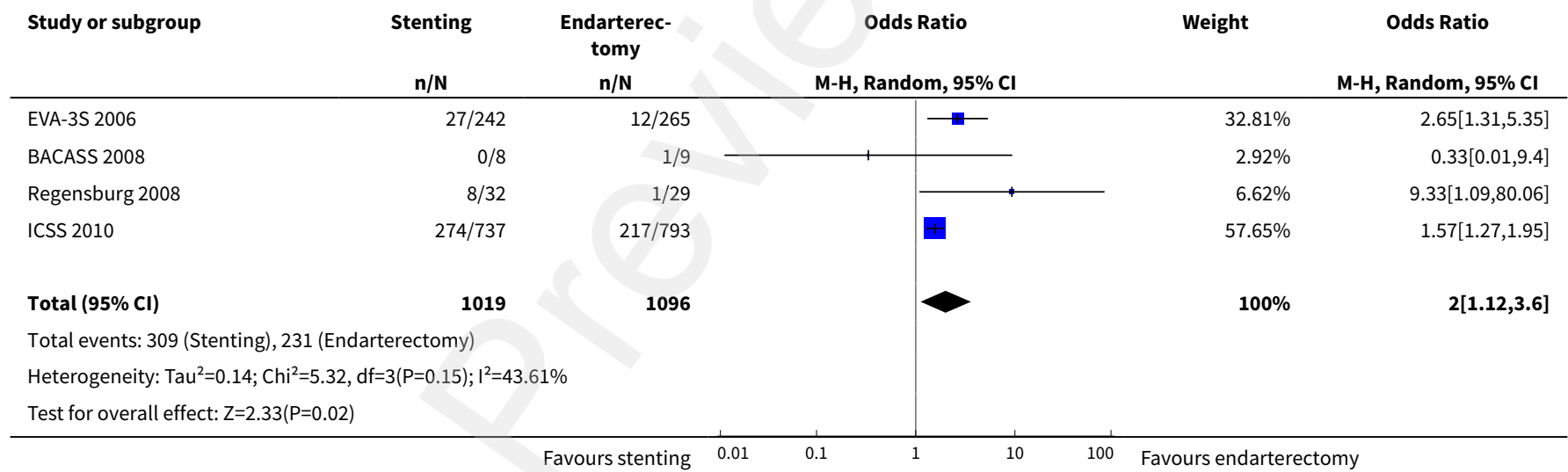

\section{Comparison 5. Access complications in stenting versus endarterectomy}

\begin{tabular}{lllll}
\hline Outcome or subgroup title & $\begin{array}{l}\text { No. of } \\
\text { studies }\end{array}$ & $\begin{array}{l}\text { No. of } \\
\text { partici- } \\
\text { pants }\end{array}$ & Statistical method & Effect size \\
\hline $\begin{array}{l}1 \text { Cranial nerve palsy within } 30 \\
\text { days of procedure }\end{array}$ & 13 & 8277 & Odds Ratio (M-H, Random, 95\% Cl) & $0.10[0.06,0.16]$ \\
\hline 1.1 Symptomatic carotid stenosis & 7 & 4965 & Odds Ratio (M-H, Random, 95\% Cl) & $0.10[0.05,0.18]$ \\
\hline 1.2 Asymptomatic carotid stenosis & 5 & 2915 & Odds Ratio (M-H, Random, 95\% Cl) & $0.09[0.03,0.27]$ \\
\hline
\end{tabular}




\begin{tabular}{lllll}
\hline Outcome or subgroup title & $\begin{array}{l}\text { No. of } \\
\text { studies }\end{array}$ & $\begin{array}{l}\text { No. of } \\
\text { partici- } \\
\text { pants }\end{array}$ & Statistical method & Effect size \\
\hline 1.3 Elevated surgical risk & 2 & 397 & Odds Ratio (M-H, Random, 95\% Cl) & $0.13[0.02,1.14]$ \\
\hline $\begin{array}{l}\text { 2 Death or neurological complica- } \\
\text { tion up to 30 days after treatment }\end{array}$ & 6 & 3642 & Odds Ratio (M-H, Random, 95\% Cl) & $0.73[0.55,0.98]$ \\
\hline 2.1 Symptomatic carotid stenosis & 5 & 3557 & Odds Ratio (M-H, Random, 95\% Cl) & $0.74[0.57,0.98]$ \\
\hline 2.2 Asymptomatic carotid stenosis & 1 & 85 & Odds Ratio (M-H, Random, 95\% Cl) & $0.13[0.01,2.59]$ \\
\hline 3 Access site haematoma & 10 & 7731 & Odds Ratio (M-H, Random, 95\% Cl) & $0.32[0.15,0.68]$ \\
\hline 3.1 Symptomatic carotid stenosis & 8 & 5012 & Odds Ratio (M-H, Random, 95\% Cl) & $0.40[0.17,0.96]$ \\
\hline 3.2 Asymptomatic carotid stenosis & 3 & 2719 & Odds Ratio (M-H, Random, 95\% Cl) & $0.14[0.02,0.90]$ \\
\hline
\end{tabular}

Analysis 5.1. Comparison 5 Access complications in stenting versus endarterectomy, Outcome 1 Cranial nerve palsy within 30 days of procedure.

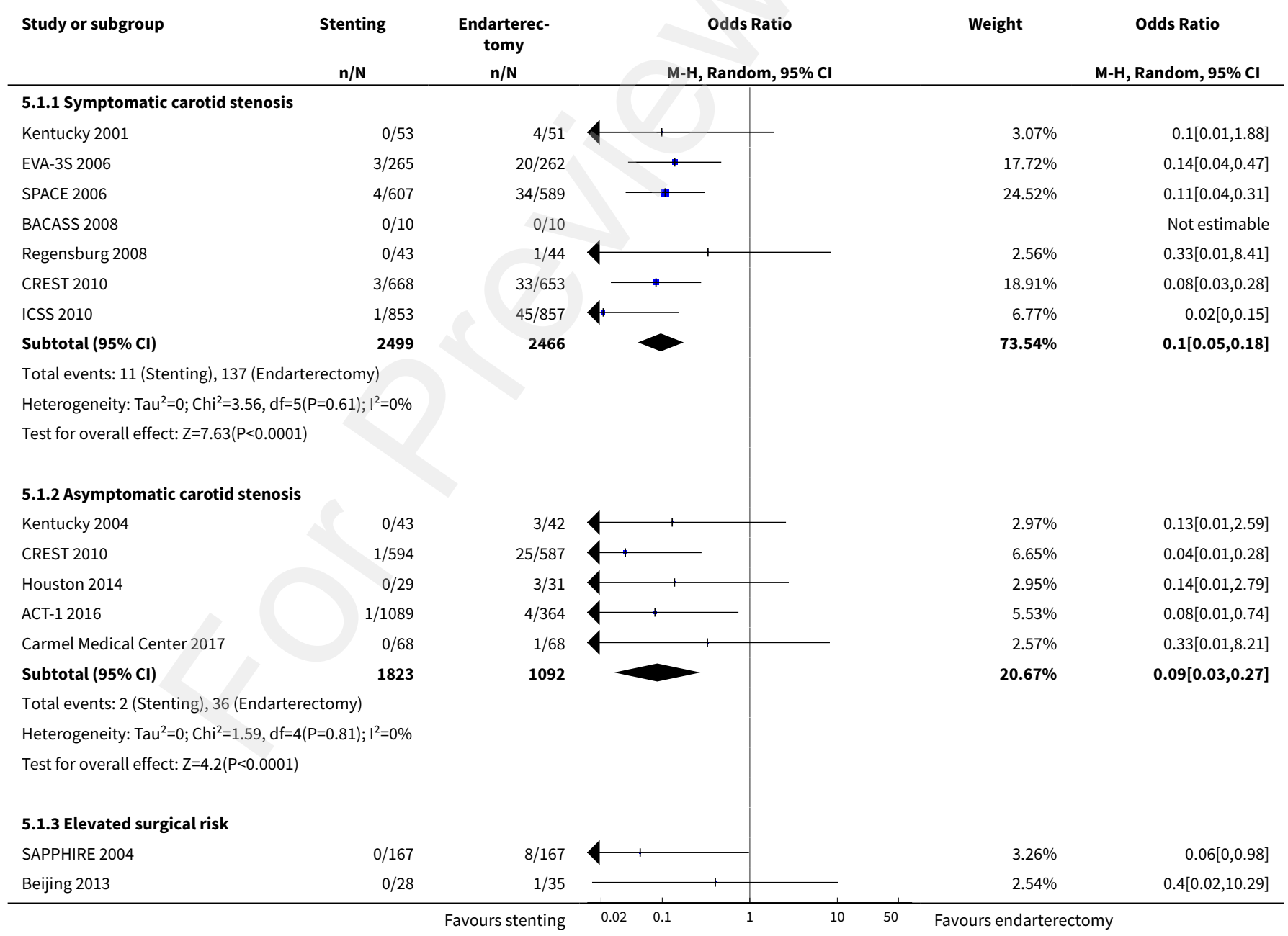




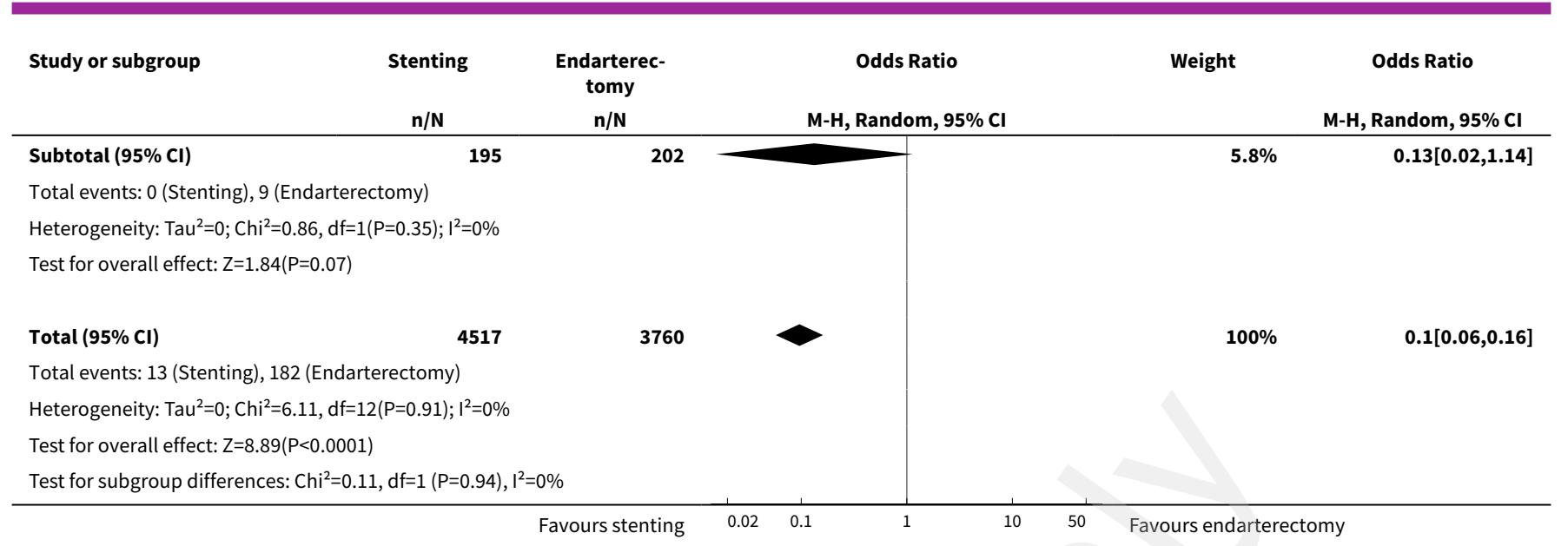

\section{Analysis 5.2. Comparison 5 Access complications in stenting versus endarterectomy, Outcome 2 Death or neurological complication up to $\mathbf{3 0}$ days after treatment.}

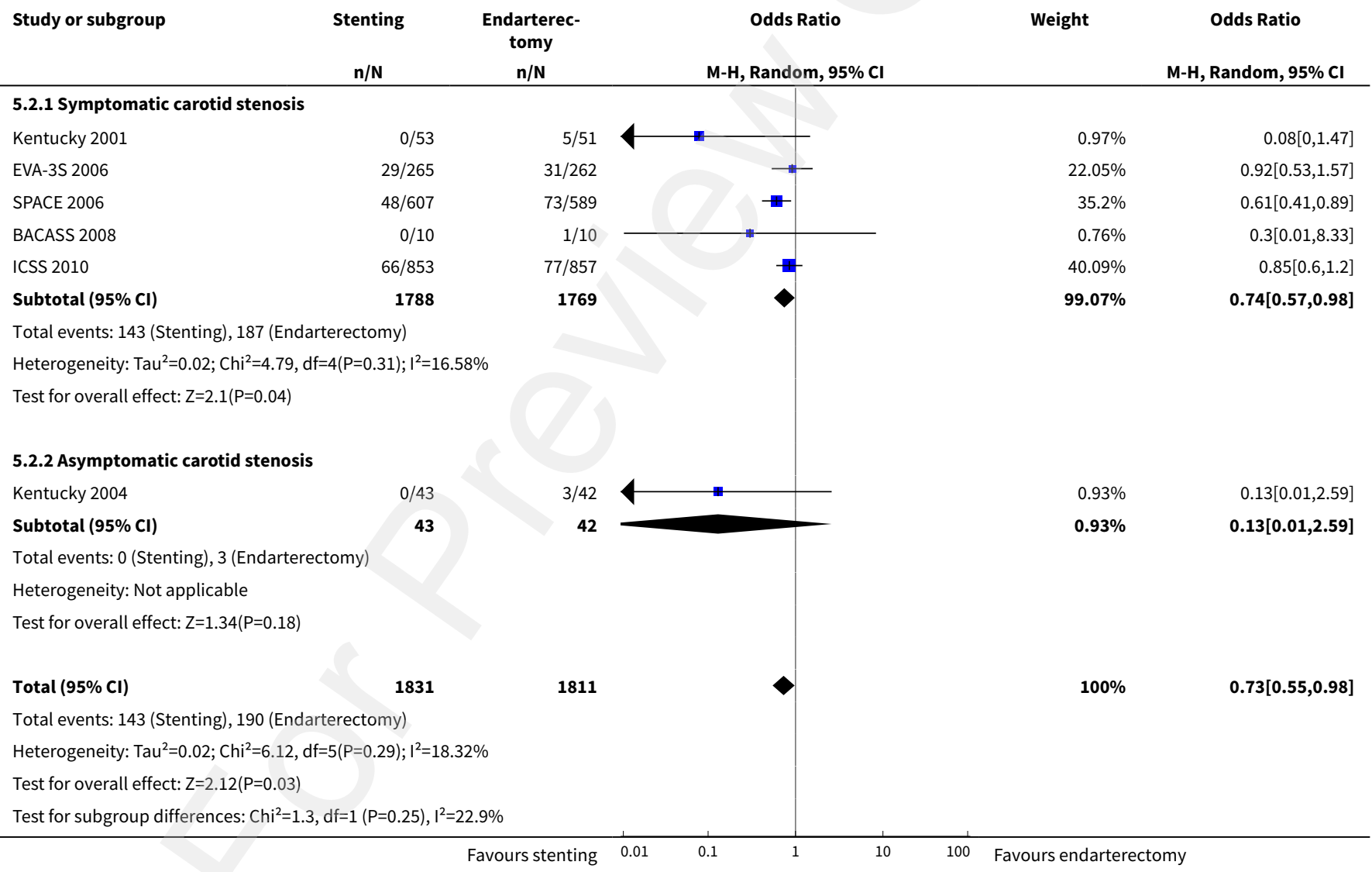


Analysis 5.3. Comparison 5 Access complications in stenting versus endarterectomy, Outcome 3 Access site haematoma.

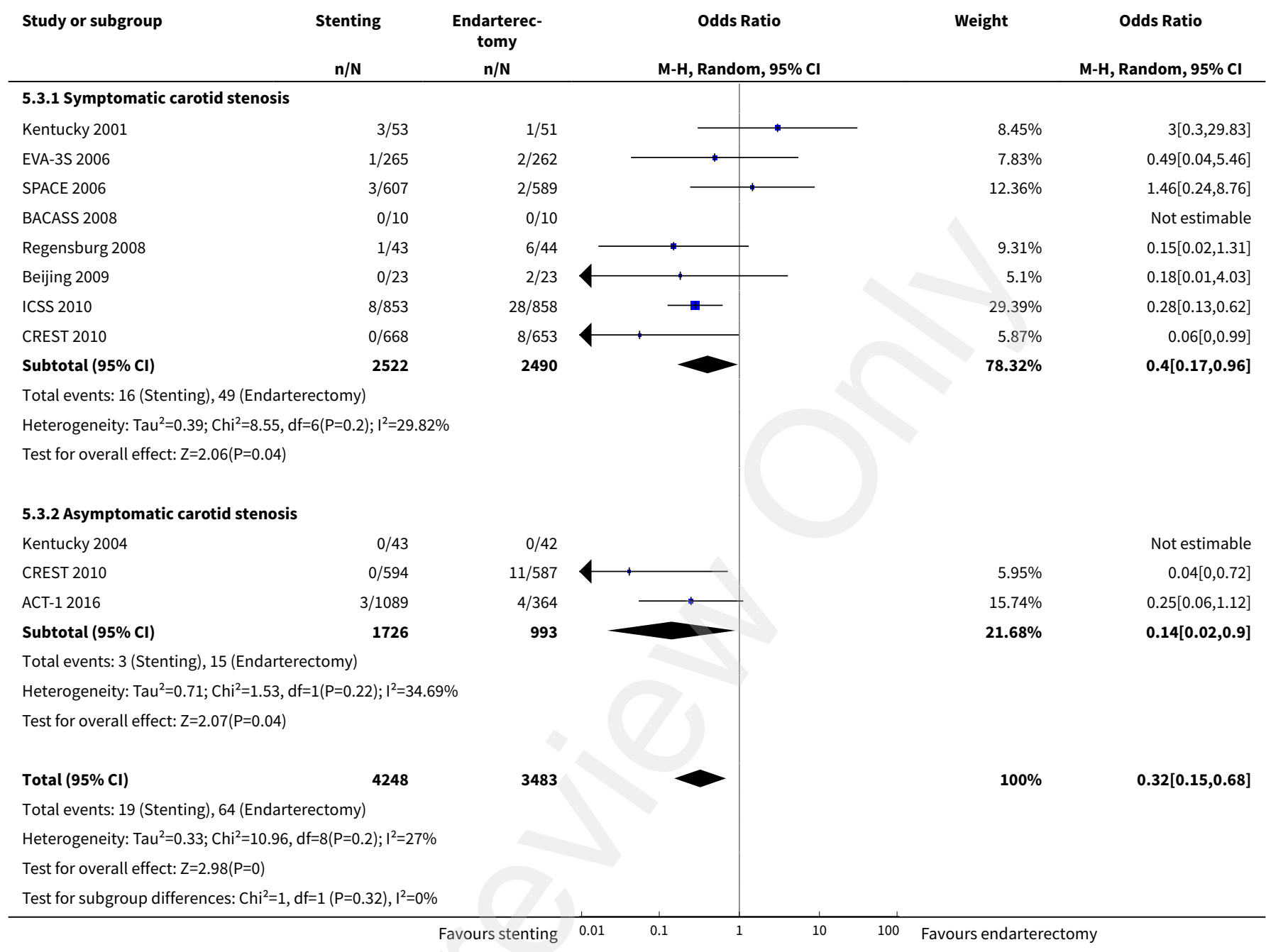

Comparison 6. Endovascular treatment or medical care in people considered not suitable for surgery

\begin{tabular}{llllll}
\hline Outcome or subgroup title & $\begin{array}{l}\text { No. of } \\
\text { studies }\end{array}$ & $\begin{array}{l}\text { No. of } \\
\text { partici- } \\
\text { pants }\end{array}$ & Statistical method & Effect size \\
\hline $\begin{array}{l}\text { 1 Death or any stroke between randomisation and } \\
\text { end of follow-up }\end{array}$ & 2 & 61 & $\begin{array}{l}\text { Odds Ratio (M-H, Random, } \\
95 \% \mathrm{Cl})\end{array}$ & $0.22[0.01,7.92]$ \\
\hline
\end{tabular}


Analysis 6.1. Comparison 6 Endovascular treatment or medical care in people considered not suitable for surgery, Outcome 1 Death or any stroke between randomisation and end of follow-up.

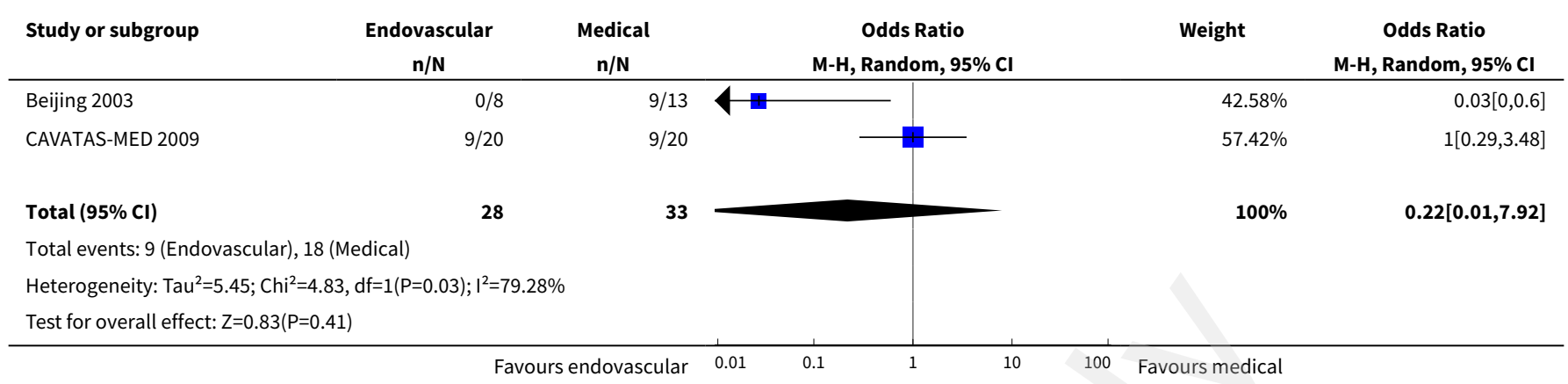

Comparison 7. Balloon angioplasty with/without stent insertion or endarterectomy for symptomatic carotid stenosis

\begin{tabular}{|c|c|c|c|c|}
\hline Outcome or subgroup title & $\begin{array}{l}\text { No. of } \\
\text { studies }\end{array}$ & $\begin{array}{l}\text { No. of } \\
\text { partici- } \\
\text { pants }\end{array}$ & Statistical method & Effect size \\
\hline $\begin{array}{l}1 \text { Death or any stroke between randomisation and } 30 \text { days } \\
\text { after treatment }\end{array}$ & 2 & 469 & $\begin{array}{l}\text { Odds Ratio (M-H, Fixed, } \\
95 \% \mathrm{Cl})\end{array}$ & $\begin{array}{l}1.76[1.06 \\
2.92]\end{array}$ \\
\hline $\begin{array}{l}2 \text { Any stroke during follow-up (periprocedural events and } \\
\text { people dying in the periprocedural period excluded) }\end{array}$ & 1 & 443 & $\begin{array}{l}\text { Odds Ratio (M-H, Random, } \\
95 \% \mathrm{Cl})\end{array}$ & $\begin{array}{l}1.85[1.06 \\
3.24]\end{array}$ \\
\hline $\begin{array}{l}3 \text { Death or any stroke between randomisation and } 30 \text { days } \\
\text { after treatment or ipsilateral stroke until the end of fol- } \\
\text { low-up }\end{array}$ & 1 & 452 & $\begin{array}{l}\text { Odds Ratio (M-H, Random, } \\
95 \% \mathrm{Cl})\end{array}$ & $\begin{array}{l}1.47[0.93 \\
2.33]\end{array}$ \\
\hline $\begin{array}{l}4 \text { Ipsilateral stroke during follow-up (periprocedural events } \\
\text { and people dying in the periprocedural period excluded) }\end{array}$ & 1 & 443 & $\begin{array}{l}\text { Odds Ratio (M-H, Random, } \\
95 \% \mathrm{Cl})\end{array}$ & $\begin{array}{l}1.33[0.62 \\
2.83]\end{array}$ \\
\hline 5 Severe $(\geq 70 \%$ ) restenosis during follow-up & 1 & 413 & $\begin{array}{l}\text { Odds Ratio (M-H, Random, } \\
95 \% \mathrm{Cl})\end{array}$ & $\begin{array}{l}3.48[1.99 \\
6.07]\end{array}$ \\
\hline
\end{tabular}

Analysis 7.1. Comparison 7 Balloon angioplasty with/without stent insertion or endarterectomy for symptomatic carotid stenosis, Outcome 1 Death or any stroke between randomisation and $\mathbf{3 0}$ days after treatment.

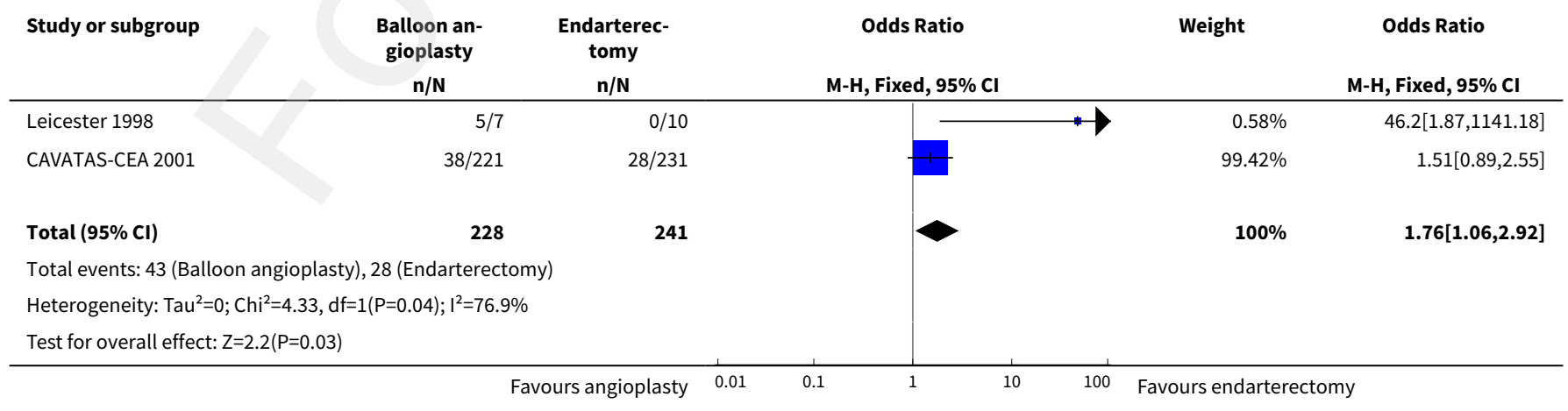


Analysis 7.2. Comparison 7 Balloon angioplasty with/without stent insertion or endarterectomy for symptomatic carotid stenosis, Outcome 2 Any stroke during followup (periprocedural events and people dying in the periprocedural period excluded).

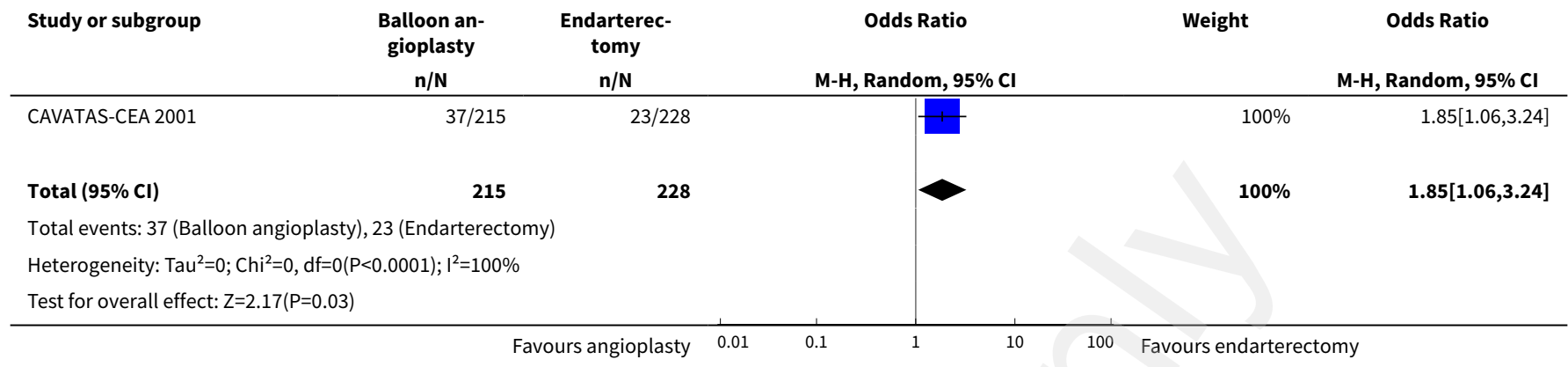

Analysis 7.3. Comparison 7 Balloon angioplasty with/without stent insertion or endarterectomy for symptomatic carotid stenosis, Outcome 3 Death or any stroke between randomisation and $\mathbf{3 0}$ days after treatment or ipsilateral stroke until the end of follow-up.

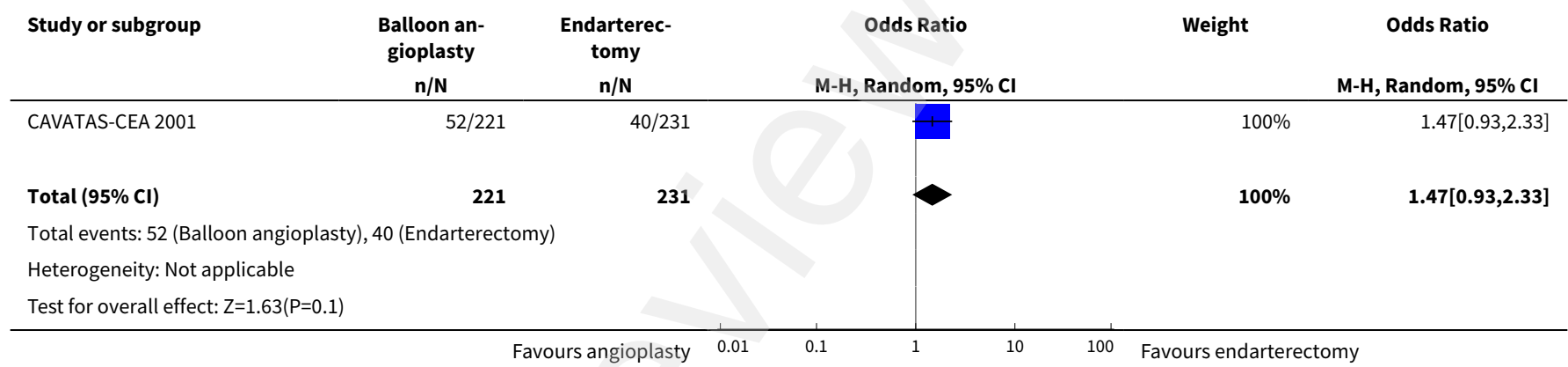

Analysis 7.4. Comparison 7 Balloon angioplasty with/without stent insertion or endarterectomy for symptomatic carotid stenosis, Outcome 4 Ipsilateral stroke during follow-up (periprocedural events and people dying in the periprocedural period excluded).

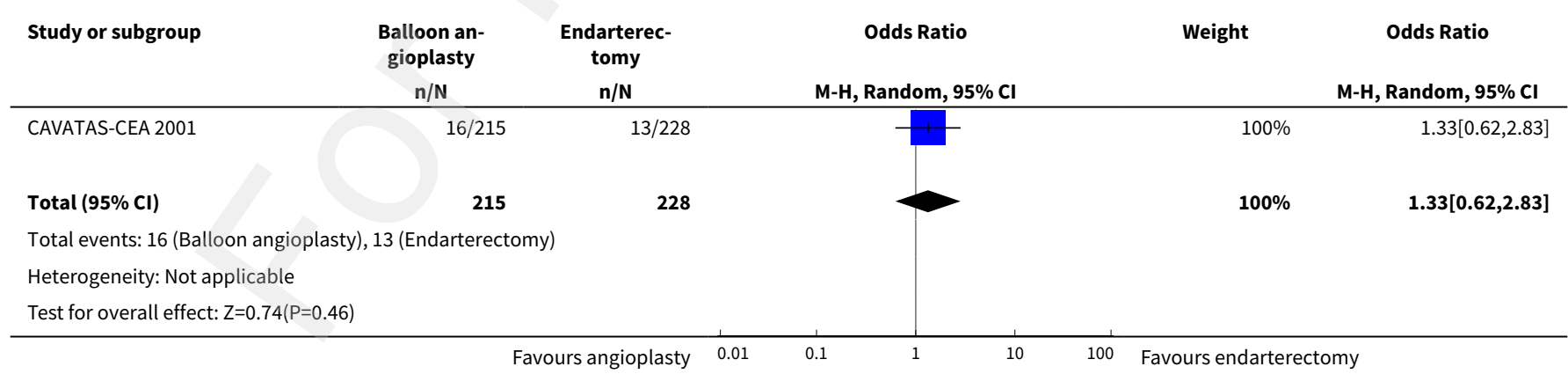


Analysis 7.5. Comparison 7 Balloon angioplasty with/without stent insertion or endarterectomy for symptomatic carotid stenosis, Outcome 5 Severe $(\geq 70 \%)$ restenosis during follow-up.

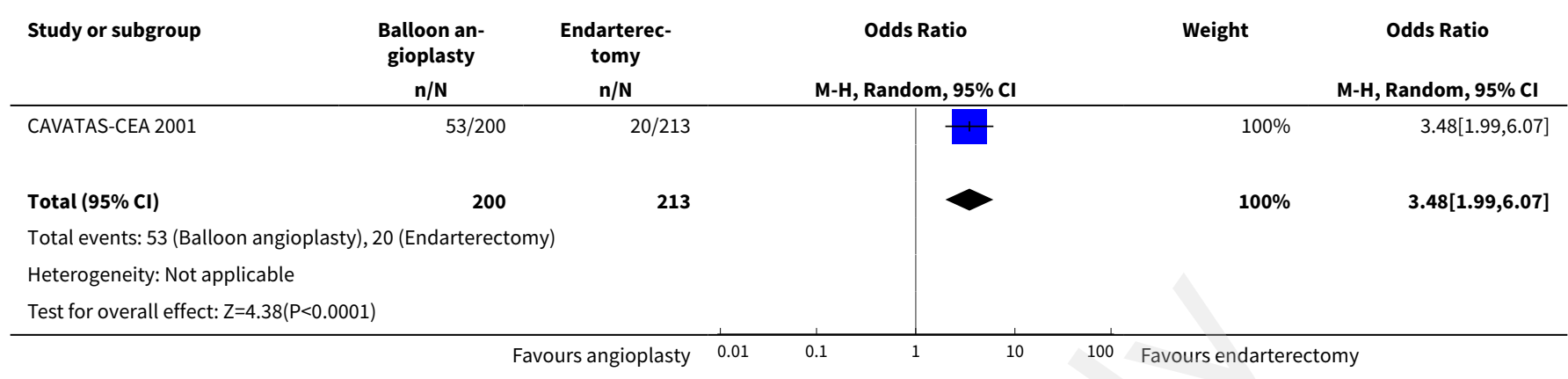

Comparison 8. Balloon angioplasty with/without stent insertion or endarterectomy for asymptomatic carotid stenosis

\begin{tabular}{lllll}
\hline Outcome or subgroup title & $\begin{array}{l}\text { No. of } \\
\text { studies }\end{array}$ & $\begin{array}{l}\text { No. of } \\
\text { partici- } \\
\text { pants }\end{array}$ & Statistical method & Effect size \\
\hline $\begin{array}{l}1 \text { Death or any stroke between randomisation and 30 days } \\
\text { after treatment }\end{array}$ & 1 & 52 & $\begin{array}{l}\text { Odds Ratio (M-H, Random, } \\
95 \% \mathrm{Cl})\end{array}$ & $\begin{array}{l}1.11[0.17, \\
7.28]\end{array}$ \\
\hline $\begin{array}{l}2 \text { Death or any stroke between randomisation and 30 days } \\
\text { after treatment or ipsilateral stroke until the end of fol- } \\
\text { low-up }\end{array}$ & 1 & 52 & $\begin{array}{l}\text { Odds Ratio (M-H, Random, } \\
95 \% \mathrm{Cl})\end{array}$ & $\begin{array}{l}1.11[0.17, \\
7.28]\end{array}$ \\
\hline
\end{tabular}

Analysis 8.1. Comparison 8 Balloon angioplasty with/without stent insertion or endarterectomy for asymptomatic carotid stenosis, Outcome 1 Death or any stroke between randomisation and 30 days after treatment.

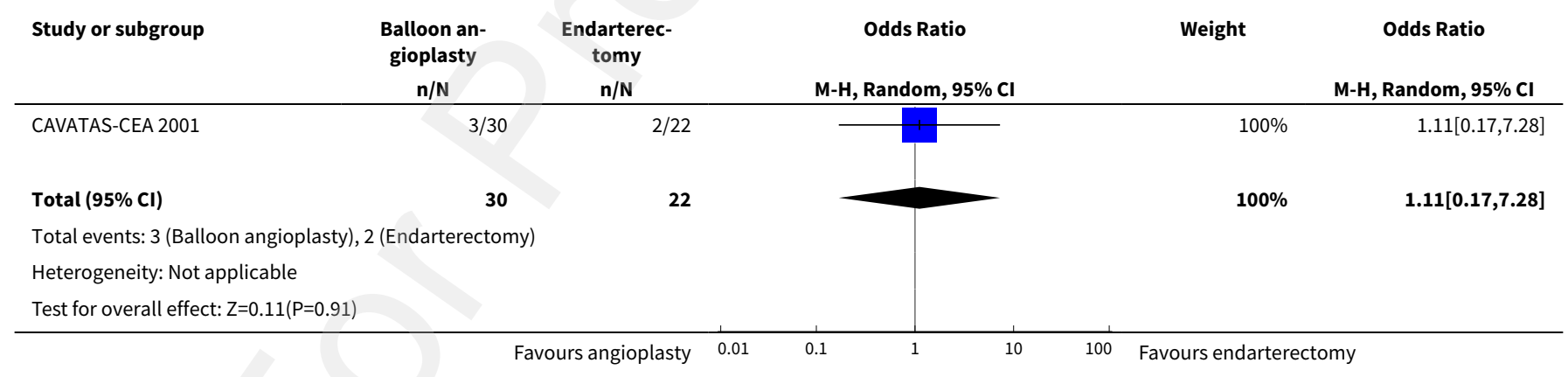


Analysis 8.2. Comparison 8 Balloon angioplasty with/without stent insertion or endarterectomy for asymptomatic carotid stenosis, Outcome 2 Death or any stroke between randomisation and $\mathbf{3 0}$ days after treatment or ipsilateral stroke until the end of follow-up.

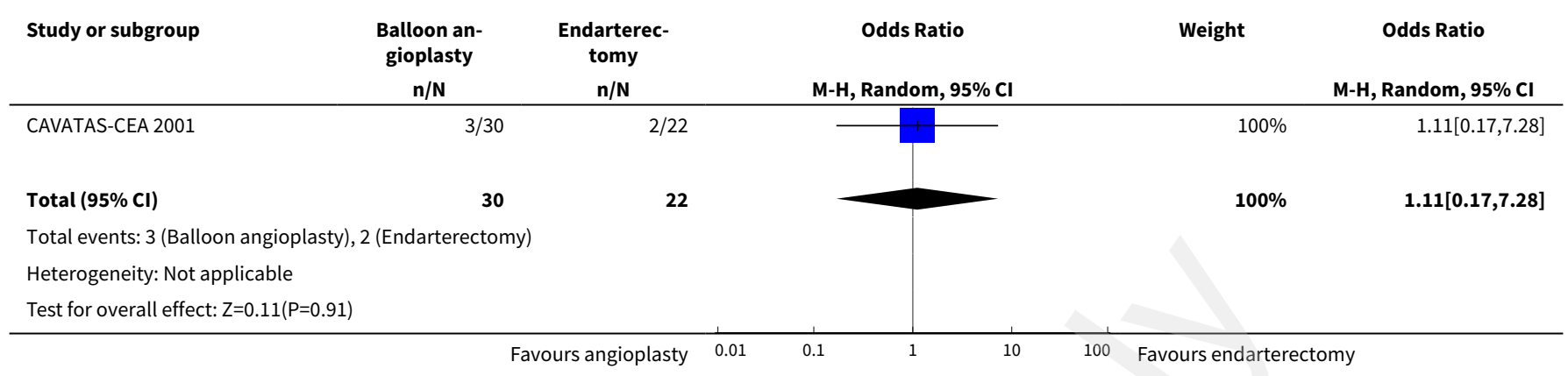

\section{APPENDICES}

\section{Appendix 1. CENTRAL search strategy}

\#1 ((carotid near/6 angioplasty) or (carotid near/6 stent $\left.\left.{ }^{\star}\right)\right):$ ti,ab,kw \#2 MeSH descriptor: [Carotid Artery Diseases] this term only \#3 MeSH descriptor: [Carotid Artery Thrombosis] this term only \#4 MeSH descriptor: [Carotid Stenosis] this term only \#5 MeSH descriptor: [Carotid Arteries] explode all trees \#6 MeSH descriptor: [Constriction, Pathologic] this term only \#7 (\#5 and \#6)

\#8 "carotid":ti,ab,kw (Word variations have been searched)

\#9 (stenosis or thrombo* or disease ${ }^{\star}$ or narrow* ${ }^{\star}$ or plaque* or $^{\text {arterioscler }}{ }^{\star}$ or atheroscler ${ }^{\star}$ ):ti,ab,kw

\#10 (\#8 and \#9)

$\# 11$ (\#2 or \#3 or \#4 or \#7 or \#10)

\#12 MeSH descriptor: [Angioplasty] explode all trees

\#13 MeSH descriptor: [Stents] this term only

\#14 (angioplasty or stent ${ }^{\star}$ or endovascular):ti,ab,kw

\#15 (balloon near/6 dilat*):ti,ab,kw

\#16 (balloon near/6 catheter $\left.{ }^{\star}\right): t i, a b, k w$

\#17 (endoluminal near/6 repair $\left.{ }^{\star}\right): t i, a b, k w$

\#18 (transluminal near/6 repair*):ti,ab,kw

$\# 19$ (\#12 or \#13 or \#14 or \#15 or \#16 or \#17 or \#18 or \#18)

\#20 (\#11 and \#19)

$\# 21 \# 1$ and \#20

\section{Appendix 2. MEDLINE (Ovid) search strategy}

1. (carotid adj10 (stent\$ or angioplasty)).tw.

2. carotid artery diseases/ or carotid artery thrombosis/ or carotid stenosis/

3. carotid arteries/ or carotid artery, common/ or carotid artery, external/ or carotid artery, internal/

4. constriction, pathologic/

5.3 and 4

6. (carotid adj5 (stenosis or thrombo $\$$ or disease $\$$ or narrow\$ or plaque $\$$ or arterioscler\$ or atheroscler\$)).tw.

7. 2 or 5 or 6

8. angioplasty/ or angioplasty, balloon/ or angioplasty, balloon, laser-assisted/ or angioplasty, laser/

9. Stents/

10. (angioplasty or stent\$ or endovascular).tw.

11. (balloon adj5 (dilat\$ or catheter\$)).tw.

12. ((endoluminal or transluminal) adj5 repair\$).tw.

13. 8 or 9 or 10 or 11 or 12

14.7 and 13

15. 1 or 14

Carotid artery stenting versus endarterectomy for treatment of carotid artery stenosis (Review) 
16. Randomized Controlled Trials as Topic/

17. random allocation/

18. Controlled Clinical Trials as Topic/

19. control groups/

20. clinical trials as topic/ or clinical trials, phase i as topic/ or clinical trials, phase ii as topic/ or clinical trials, phase iii as topic/ or clinical trials, phase iv as topic/

21. double-blind method/

22. single-blind method/

23. Placebos/

24. placebo effect/

25. Drug Evaluation/

26. Research Design/

26. randomized controlled trial.pt.

27. controlled clinical trial.pt.

28. clinical trial.pt.

29. random\$.tw.

30. (controlled adj5 (trial\$ or stud\$)).tw.

31. (clinical\$ adj5 trial\$).tw.

32. ((control or treatment or experiment\$ or intervention or surgical) adj5 (group\$ or subject\$ or patient\$)).tw.

33. (quasi-random $\$$ or quasi random $\$$ or pseudo-random $\$$ or pseudo random\$).tw.

34. ((control or experiment\$ or conservative) adj5 (treatment or therapy or procedure or manage\$)).tw.

35. ((singl\$ or doubl\$ or tripl\$ or trebl\$) adj5 (blind\$ or mask\$)).tw.

36. (coin adj5 (flip or flipped or toss\$)).tw.

37. latin square.tw.

38. versus.tw.

39. controls.tw.

40. or/17-39

41.16 and 40

42. limit 41 to humans

\section{Appendix 3. Embase (Ovid) search strategy}

1. carotid angioplasty/ or carotid artery stenting/

2. (carotid adj10 (stent\$ or angioplasty)).tw.

3. carotid artery disease/ or carotid artery obstruction/ or carotid artery thrombosis/ or internal carotid artery occlusion/ or carotid atherosclerosis/

4. carotid artery/ or common carotid artery/ or external carotid artery/ or internal carotid artery/

5. "stenosis, occlusion and obstruction"/ or stenosis/ or artery constriction/

6. 4 and 5

7. (carotid adj5 (stenosis or thrombo $\$$ or disease $\$$ or narrow\$ or plaque $\$$ or arterioscler\$ or atheroscler\$)).tw.

8.3 or 6 or 7

9. angioplasty/ or laser angioplasty/ or percutaneous transluminal angioplasty/

10. balloon dilatation/

11. stent/

12. (angioplasty or stent\$ or endovascular).tw.

13. (balloon adj5 (dilat\$ or catheter\$)).tw.

14. ((endoluminal or transluminal) adj5 repair\$).tw.

15.9 or 10 or 11 or 12 or 13 or 14

16. 8 and 15

17. 1 or 2 or 16

18. Randomized Controlled Trial/

19. Randomization/

20. Controlled Study/

21. control group/

22. exp Clinical Trial/

23. Double Blind Procedure/

24. Single Blind Procedure/

25. latin square design/

26. Parallel Design/

27. "types of study"/

28. research subject/

29. Comparative Study/ 
30. random\$.tw.

31. (controlled adj5 (trial\$ or stud\$)).tw.

32. (clinical\$ adj5 trial\$).tw.

33. ((control or treatment or experiment\$ or intervention or surgical) adj5 (group\$ or subject\$ or patient\$)).tw.

34. (quasi-random $\$$ or quasi random\$ or pseudo-random $\$$ or pseudo random\$).tw.

35. ((control or experiment $\$$ or conservative) adj5 (treatment or therapy or procedure or manage $\$)$ ).tw.

36. ((singl\$ or doubl\$ or tripl\$ or trebl\$) adj5 (blind\$ or mask\$)).tw.

37. (coin adj5 (flip or flipped or toss\$)).tw.

38. latin square.tw.

39. versus.tw.

40. controls.tw.

41. or $/ 18-40$

42. 17 and 41

43. limit 42 to human

Appendix 4. Science Citation Index search strategy

TS=carotid AND TS=(angioplasty or balloon or stent ${ }^{\star}$ or endovascular) AND TS=(trial ${ }^{\star}$ or random ${ }^{\star}$ or versus)

\section{Appendix 5. Stroke Trials Registry}

carotid artery

\section{Appendix 6. US National Institutes of Health Ongoing Trials Register ClinicalTrials.gov}

( angioplasty OR stenting OR balloon ) AND ( Carotid Artery Diseases OR Carotid Stenosis) [DISEASE]

\section{Appendix 7. ISRCTN Registry}

carotid artery

\section{Appendix 8. World Health Organization International Clinical Trials Registry Platform}

Condition: carotid

Intervention: angioplasty OR stenting OR balloon

Recruitment status is: ALL

Phases are: ALL

WHAT'S NEW

\begin{tabular}{|c|c|c|}
\hline Date & Event & Description \\
\hline 12 January 2019 & New search has been performed & $\begin{array}{l}\text { For the } 2018 \text { update of this review, there were new long-term } \\
\text { follow-up data from five trials included in the previous version } \\
\text { (Kentucky 2001; Kentucky 2004; EVA-3S 2006; CREST 2010; ICSS } \\
\text { 2010). Results from three trials cited in the previous version as } \\
\text { ongoing studies have now been published and included in this } \\
\text { update (ACT-1 2016; SPACE-2 2016; Carmel Medical Center 2017). } \\
\text { In addition, we identified three new studies which met the in- } \\
\text { clusion criteria (Beijing 2013; Houston 2014; Ostrava 2014). We } \\
\text { analysed the data of participants with symptomatic and asymp- } \\
\text { tomatic carotid stenosis from one previously included trial sep- } \\
\text { arately (CREST 2010). Moreover, we analysed data from partici- } \\
\text { pants considered at elevated risk for complications from surgery } \\
\text { separately (SAPPHIRE 2004; Beijing 2013). }\end{array}$ \\
\hline
\end{tabular}

The present update of this review includes data for 9753 participants from 22 trials. The title and objectives of this review have been amended to reflect changes in endovascular technique and terminology. Therefore, the current review focuses on the term carotid stenting rather than the term endovascular treatment. In addition, we included studies investigating cognitive outcomes after endarterectomy and stenting, although meta-analysis of 


$\begin{array}{lll}\text { Date Event Description } & \text { Denten }\end{array}$

these outcomes was not possible (SPACE 2006; ICSS 2010; Houston 2014). We had access to individual patient data from five trials (EVA-3S 2006; SPACE 2006; CREST 2010; ICSS 2010; Ostrava 2014). The Background, Results, Description of studies, Risk of bias in included studies, and Discussion sections have been substantially revised to incorporate the new data. We have updated the Risk of bias in included studies tables, used GRADE to assess the overall certainty of the evidence, and included 'Summary of findings' tables showing our primary safety and primary combined safety and long-term efficacy outcomes (Summary of findings for the main comparison; Summary of findings 2).

12 January $2019 \quad$ New citation required and conclusions have changed
The conclusion of the review is that there is high-certainty evidence for the comparison of short-term and long-term outcomes between carotid artery stenting and carotid endarterectomy in people with symptomatic carotid stenosis. Periprocedural stroke or death occurs more often after stenting than endarterectomy. However, this difference is strongly dependent on age: shortterm outcomes were similar with both treatments in people below the age of 70 years. Stenting and endarterectomy are equally effective in preventing recurrent stroke in the long term. For people with asymptomatic carotid stenosis, there is moderate-certainty evidence on short-term and long-term outcomes. The available evidence showed a strong trend towards a higher risk of periprocedural stroke or death after stenting than endarterectomy, but no evidence for a difference in occurrence of stroke in the long term. More data from randomised trials comparing stenting versus endarterectomy including long-term follow-up are needed.

\section{HISTORY}

Protocol first published: Issue 4, 1997

Review first published: Issue 4, 1997

\begin{tabular}{lll}
\hline Date & Event & Description \\
\hline 26 April 2012 & $\begin{array}{l}\text { New citation required and conclusions } \\
\text { have changed }\end{array}$ & $\begin{array}{l}\text { The conclusion of the review is that the comparison of short- } \\
\text { term risk of stroke or death clearly favours endarterectomy over } \\
\text { endovascular treatment. However, this effect is strongly depen- } \\
\text { dent on age: outcomes were similar with both treatments in pa- } \\
\text { tients below the age of } 70 \text { years. Other outcome events, such } \\
\text { as myocardial infarction, cranial nerve palsy and haematoma, } \\
\text { favoured endovascular treatment. }\end{array}$ \\
& $\begin{array}{ll}\text { For the 2012 update of this review, results from three trials cit- } \\
\text { ed in the previous version as ongoing studies have now been }\end{array}$ \\
& $\begin{array}{l}\text { published and included (Regensburg 2008; CREST 2010; ICSS } \\
\text { 2010). We have identified and included one additional trial which } \\
\text { met the inclusion criteria (Beijing 2009). We analysed the data } \\
\text { of patients with symptomatic and asymptomatic carotid steno- } \\
\text { sis from one previously included trial separately (CAVATAS-CEA } \\
\text { 2001). We analysed data from another previously included tri- } \\
\text { al separately because the trial enrolled patients considered at } \\
\text { increased risk for complications with surgery (SAPPHIRE 2004). } \\
\text { New long-term follow-up data were available from three previ- }\end{array}$ \\
\end{tabular}




\begin{tabular}{lll}
\hline Date Event $\quad$ Description
\end{tabular}

ously included trials (CAVATAS-CEA 2001; EVA-3S 2006; SPACE

2006), as well as from one new trial (CREST 2010). The present update of this review includes data for a total of 7572 patients from 16 trials. The objectives of the review have been amended to include comparisons of outcomes according to age and sex. We had access to individual patient data from five trials (CAVATAS-CEA 2001; EVA-3S 2006; SPACE 2006; BACASS 2008; ICSS 2010) and used reported outcomes of individual patients (Leicester 1998) or patient subgroups (CREST 2010) from two other trials, to perform subgroup analyses according to age and sex. The Background, Description of studies, Risk of bias in included studies, Results and Discussion sections have been substantially revised to incorporate the new data. We have updated the 'Risk of bias' tables using the new tool in Review Manager 5.1. requirements.

\section{CONTRIBUTIONS OF AUTHORS}

MDM and LHB selected studies for inclusion in this review.

MDM and PL extracted outcome data.

MDM drafted the review.

LHB was the first author of the previous version of this review and critically reviewed the manuscript.

MMB contributed to the critical revision of the review and gave final approval of the version to be published.

\section{DECLARATIONS OF INTEREST}

MDM: none.

PL: was involved as local principal investigator (PI) in the CAVATAS-CEA 2001, CAVATAS-MED 2009, and ICSS 2010 trials. He was a member of the steering committee as well as PI in the BACASS trial. He was not involved in data extraction from ICSS and CAVATAS trials.

MMB: was the chief investigator of three completed trials (CAVATAS-CEA 2001; CAVATAS-MED 2009; ICSS 2010), and one ongoing trial (EVA-3S 2006). He was not involved in data extraction from ICSS and CAVATAS. 
LHB: was a member of the ICSS writing committee. He contributed to the analysis of data in CAVATAS-CEA 2001, CAVATAS-MED 2009, and ICSS 2010 trials, and is a member of the ECST-2 and ACST-2 steering committees. He was not involved in data extraction from CAVATASCEA 2001, CAVATAS-MED 2009, and ICSS 2010 trials.

\section{SOURCES OF SUPPORT}

\section{Internal sources}

- No sources of support supplied

\section{External sources}

- The Stroke Association, UK.

- Department of Health, UK.

- Reta Lila Weston Trust for Medical Research, UK.

- Medical Research Council, UK.

- National Institute of Health Research, UK.

- Swiss National Science Foundation, Switzerland.

\section{DIFFERENCES BETWEEN PROTOCOL AND REVIEW}

We amended the title and objectives of this review to reflect changes in endovascular technique and terminology. Therefore, the current review focused on the term carotid stenting rather than the term endovascular treatment. Additionally MDM was included as a new author of the latest version of this review.

We separated outcome measures into primary ones reflecting the main research questions as specified in the original version of this review (Crawley 1997), and secondary ones reflecting additional research questions stated in the original version or added during previous updates (Coward 2004; Ederle 2007; Bonati 2012). According to the original version of this review, the primary outcome measure to evaluate treatment safety was defined as death or any stroke occurring in the periprocedural period. In order to allow an intention-to-treat approach (i.e. including outcomes in all participants who were randomised and subsequently followed in the contributing trials and comparing outcomes according to the randomly assigned treatment), we defined the periprocedural period as the period between randomisation and 30 days after treatment (or 30 days after randomisation in participants not receiving treatment). Hence, there were small differences in numbers of outcome events for some of the included trials between the current update and previous versions of this review (Coward 2004; Ederle 2007; Bonati 2012). We defined the combined primary safety and long-term efficacy outcome measure as death or any stroke occurring in the periprocedural period, or ipsilateral stroke thereafter, because this outcome could be extracted from all large trials included in this review. We added myocardial infarction as a separate periprocedural outcome measure.

The comparisons of outcomes according to pretrial experience in endovascular treatment, age, and sex included in the previous version of this review have been updated. We extracted individual participant data directly from the databases of six trials in order to perform subgroup analysis according to age (EVA-3S 2006; SPACE 2006; BACASS 2008; CREST 2010; ICSS 2010; Ostrava 2014).

We included data from extensive follow-up periods of up to 10 years in this review from five trials (Kentucky 2001; Kentucky 2004; EVA-3S 2006; CREST 2010; ICSS 2010).

In addition, we added new evidence from five trials including participants with asymptomatic carotid stenosis in this update of this review (Houston 2014; Ostrava 2014; ACT-1 2016; SPACE-2 2016; Carmel Medical Center 2017).

We also included data on cognitive performance after carotid endarterectomy or stenting, although meta-analyses of these results were not possible due to substantial heterogeneity in the tests used to assess cognitive performance as well as differences in time points at which cognitive performance after treatment was assessed.

We separated data from two trials from the other trials because, unlike all the other trials, these trials enrolled participants considered at increased risk for complications from surgery (SAPPHIRE 2004; Beijing 2013). 